\title{
[1,3]-Claisen Rearrangement via Removable Functional Group Mediated Radical Stabilization
}

Md Nirshad Alam, ${ }^{\text {a, }}$ Soumya Ranjan Dash, ${ }^{\mathrm{b}, \mathrm{c}}$ Anirban Mukherjee, ${ }^{\mathrm{a}}$ Satish Pandole, ${ }^{\mathrm{a}}$ Udaya Kiran Marelli, Kumar Vanka, ${ }^{\mathrm{b}, \mathrm{c}}$ and Pradip Maity ${ }^{\mathrm{a} *}$

${ }^{a}$ Organic Chemistry Division, CSIR-National Chemical Laboratory, Pune-411008, India. ${ }^{b}$ Academy of Scientific and Innovative Research (AcSIR), Ghaziabad-201002, India. ${ }^{c}$ Physical and Material Chemistry Division, CSIR-National Chemical Laboratory, Pune 411008, India 


\section{Table of Contents}

General Information

Synthesis of starting materials

General procedures for $[1,3]$ rearrangement

Mechanistic and kinetic studies

Functionalization of $[1,3]$ products

S-13

Comutational methods

Compound characterizations

References

NMR Spectra 


\section{Experimental Procedures}

\section{General Information}

The thermal [1,3] rearrangement reactions and all starting material synthesis were carried out with anhydrous and oxygen-free solvents in flame-dried glasswares under an inert atmosphere. THF was dried over sodium before use. All other solvents were purchased anhydrous and stored under argon over $4 \AA$ molecular sieves. Commercially available reagents were purchased and used as obtained. Analytical thin-layer chromatography was performed on glass plates pre-coated with silica gel (Silica Gel $60 \mathrm{~F}_{254}$; Merck). Plates were visualized using UV light $(\lambda=254 \mathrm{~nm})$ and then stained with either aqueous basic potassium permanganate $\left(\mathrm{KMnO}_{4}\right)$ or $p$ anisaldehyde and developed upon heating in Hitachi heat gun. Flash chromatography was performed using silica gel (Merck and Spectrochem, 230-400 mesh), eluting with solvents as indicated. Flash column was performed using Sebo aquarium air pump (SB-548A). ${ }^{1} \mathrm{H}$ and ${ }^{13} \mathrm{C}$ spectras were acquired in deuterated solvents at room temperature on Bruker: Ultrashield AV $400 \mathrm{MHz}$, Ultrashield AV $500 \mathrm{MHz}$, Ultrashield Neo 400, Ultrashield Neo 500, Ultrashield AV $200 \mathrm{MHz}$ and JEOL 400 spectrometer. Chemical shifts $(\delta)$ are reported for ${ }^{1} \mathrm{H}$ NMR in ppm from TMS as internal standard and ${ }^{13} \mathrm{C}$ from the residual solvent peak. ${ }^{1} \mathrm{H}$ NMR spectra are reported as follows: chemical shift $(\delta \mathrm{ppm})$, multiplicity, coupling constant $(\mathrm{Hz})$, and integration. Data for ${ }^{13} \mathrm{C}$ NMR spectra are reported in terms of chemical shift $(\delta \mathrm{ppm})$. Melting points were checked in Buchi Melting Point B-540 instrument and reported in ${ }^{\circ} \mathrm{C}$. FT-IR were analyzed in Bruker ALPHA instrument and reported as $\mathrm{cm}^{-1}$. High resolution (HRMS) mass spectral analyses were recorded on a Thermo Scientific Q-Exactive, Accela 1250 pump.

\section{Preparation of Starting Materials:}

\section{A: 2-Aminothiophenol protection of $\alpha$-hydoxyacid: ${ }^{1}$}

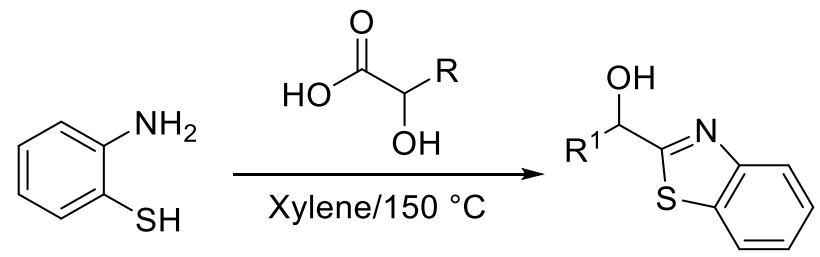

In an oven dried $50 \mathrm{ml}$ round bottom flask with a magnetic bar, 2-Aminothiophenol (1.28g, $10 \mathrm{mmol}, 1.0$ equiv.), $\alpha$-hydroxy acid (3 equiv., $30 \mathrm{mmol}$ ) were taken in $25 \mathrm{ml} \mathrm{Xylene}(0.4 \mathrm{M})$ at room temperature. The reaction was stirred with heating in a oil bath at $150^{\circ} \mathrm{C}$ for overnight and after completion of the reaction monitored by TLC, solvent removal under reduced pressure in rotary evaporator gave the crude product. The crude was further purified by column chromatography on silica gel using ethylacetate-pet ether (6 - 8\% EA/PE) as eluent to obtain the desired product.

\section{B:General scheme for the synthesis of substituted benzyl bromide:}

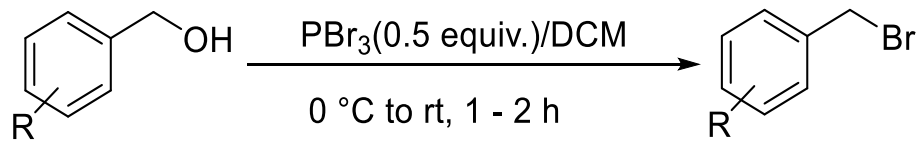

In a flame dried $50 \mathrm{ml}$ round bottom flask with a magnetic bar, alcohol (2 mmol, 1 equiv.) was taken in dry DCM $(10 \mathrm{ml}, 0.2 \mathrm{M})$ and cooled to $0{ }^{\circ} \mathrm{C}$ under argon atmosphere. To the solution 0.5 equiv. $\mathrm{PBr}_{3}$ was added dropwise and stirred the solution at $0{ }^{\circ} \mathrm{C}$ to room temperature for its completion judged by TLC. The reaction was quenched by pouring the reaction mixture into another canonical flask containing ice-cold water. Organic layer was separated and washed with saturated $\mathrm{NaHCO}_{3}$ solution followed by drying over anhydrous $\mathrm{Na}_{2} \mathrm{SO}_{4}$. Solvent removal under reduced pressure gave the crude product which was used without further purification for the next step. 


\section{C: General procedure for the synthesis of ether $(3 a-x):^{2}$}

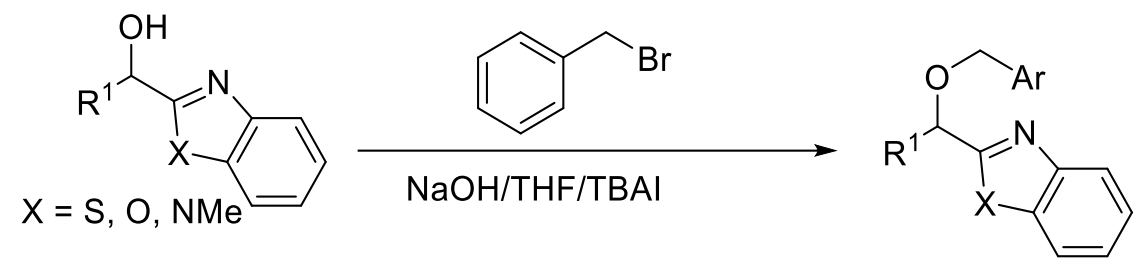

In an oven dried $50 \mathrm{ml}$ round bottom flask with a magnetic bar, alcohol ( $0.5-2.5 \mathrm{mmol}, 1.0$ equiv.), $\mathrm{NaOH}$ (10 equiv.), and tetrabutylammonium iodide ( 0.3 equiv.) were taken and cooled to $0{ }^{\circ} \mathrm{C}$ under argon atmosphere. A solution of substituted benzyl bromide $\left(1.5\right.$ equiv) in dry THF $(0.1 \mathrm{M})$ was added to the reaction mixture at $0{ }^{\circ} \mathrm{C}$ and sealed with electrical tape. The reaction mixture was stirred at room temperature for 24 hours, and quenched with saturated $\mathrm{NH}_{4} \mathrm{Cl}$ solution. The organic layer was extracted with EtOAc (3 times) and combined organic layer was dried over anhydrous $\mathrm{Na}_{2} \mathrm{SO}_{4}$. The solvent removal under reduced pressure gave the crude product which was further purified by column chromatography on silica gel using ethylacetate-pet ether ( 2 - 3\% EA/PE) as eluent to obtain the desired product.

\section{(D) General procedure for Synthesizing the compound S13a-c and 3w-y}

D-1

1.n-BuLi(1.6M, 1 equiv.)

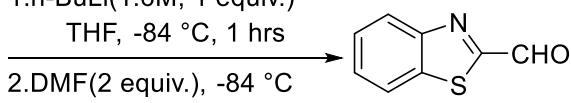

$4 \mathrm{~h}$
D-2

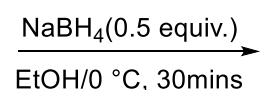
$53 \%$ yiled D-4

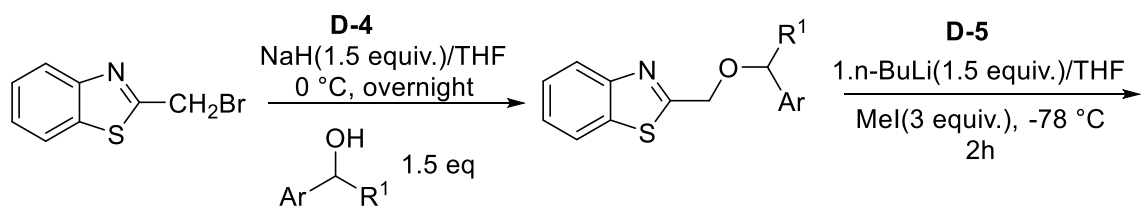

D-3

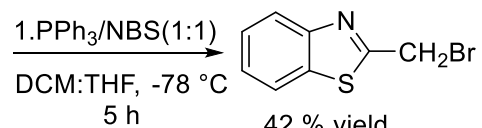

Steps D-1, ${ }^{3} \mathbf{D}-\mathbf{2},{ }^{3}$ and $\mathbf{D}-\mathbf{3}^{4}$ are performed according to the literature procedures.

D-4: In a flame dried $50 \mathrm{ml}$ round bottom flask with a magnetic bar, $\mathrm{NaH}$ (188 $\mathrm{mg}, 4.69 \mathrm{mmol}, 1.5 \mathrm{equiv}$.$) in dry$ THF $(6 \mathrm{ml}, 0.8 \mathrm{M})$ was taken and sealed with insulating tape. The reaction was cooled to $0{ }^{\circ} \mathrm{C}$ under argon atmoshphere and alcohol (4.69 mmol, 1.5 equiv.) in $4 \mathrm{ml}$ THF $(1.2 \mathrm{M})$ was added dropwise with continued stirring for $30 \mathrm{~min}$. Next, S12 (710 mg, $3.14 \mathrm{mmol}, 1.0$ equiv.) in $4 \mathrm{ml}$ THF (0.8 M) was added dropwise to the reaction mixture at $0{ }^{\circ} \mathrm{C}$ and continued stirring for overnight. The consumption of starting material was monitored by TLC and reaction mixture was quenched with saturated $\mathrm{NH}_{4} \mathrm{Cl}$ solution at $0{ }^{\circ} \mathrm{C}$. The organic layer was extracted with EtOAc (3 times) and combined organic layer dried over anhydrous $\mathrm{Na}_{2} \mathrm{SO}_{4}$. The solvent removal under reduced pressure gave the crude product which was further purified by column chromatography on silica gel using ethylacetate-pet ether (1- 2\% EA/PE) as eluent to obtain the desired product.

D-5: In a flame dried $25 \mathrm{ml}$ round bottom flask with a magnetic bar, $\mathbf{S 1 3}$ (1 mmol, 1.0 equiv.) in dry THF (5 ml, $0.2 \mathrm{M})$ was taken and sealed with insulating tape. The reaction was cooled to $-78{ }^{\circ} \mathrm{C}$ under argon atmoshphere and $\mathrm{n}-\mathrm{BuLi}$ (1.6M in Hexane, 1.5 equiv.) was added dropwise with continued stirring for $30 \mathrm{~min}$. Next, MeI ( 3.0 mmol, 3.0 equiv.) was added dropwise to the reaction container at $-78{ }^{\circ} \mathrm{C}$ and continued stirring for $2 \mathrm{~h}$. The consumption of starting material was monitored by TLC and reaction mixture was quenched with saturated $\mathrm{NH}_{4} \mathrm{Cl}$ solution at $-78{ }^{\circ} \mathrm{C}$. The organic layer was extracted with EtOAc (3 times) and combined organic layer dried over anhydrous $\mathrm{Na}_{2} \mathrm{SO}_{4}$. The solvent removal under reduced pressure gave the crude product which was further purified by column chromatography on silica gel using ethylacetate-pet ether (1 - $1.5 \% \mathrm{EA} / \mathrm{PE})$ as eluent to obtain the desired product. 
<smiles>[R]c1cccc(COC(C)c2nc3ccccc3s2)c1</smiles>

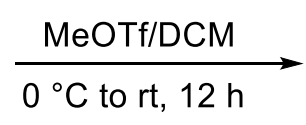<smiles>[R]Cc1cccc(COC(C)c2nc3ccccc3s2)c1</smiles>

In a flame dried $25 \mathrm{ml}$ round bottom flask with a magnetic bar, ether $(0.371 \mathrm{mmol}, 1.0$ equiv.) was taken in dry DCM $(3 \mathrm{ml}, 0.12 \mathrm{M})$ and cooled to $0{ }^{\circ} \mathrm{C}$ under argon atmosphere. To the solution MeOTf $(0.556 \mathrm{mmol}, 1.5$ equiv.) was added dropwise and slowly warming to room temperature with continued stirring for $12 \mathrm{~h}$. Upon consumption of starting material (by TLC) the reaction mixture was directly loaded on silica column and purified by $(2-3 \% \mathrm{MeOH} / \mathrm{DCM})$ as eluent to obtain the desired product.

\section{F. General procedure for facial $[1,3]$ rearrangement reactions}<smiles>[R]C([Al])OC([R])c1nc2ccccc2s1</smiles><smiles>[R]C(OC([R])C)=C1Sc2ccccc2N1C</smiles><smiles>CO[AsH3]</smiles><smiles>[R]C(=O)C1(C([R])[AlH2])Sc2ccccc2N1C</smiles>

In a flame dried $25 \mathrm{ml}$ round bottom flask with a magnetic bar, ether $(0.2 \mathrm{mmol}, 1$ equiv. $)$ and $\mathrm{K}_{2} \mathrm{CO}_{3}(0.4 \mathrm{mmol}$, 2 equiv.) in dry DCM $(1 \mathrm{ml}, 0.2 \mathrm{M})$ were taken and sealed with electrical tape and degassed for $10 \mathrm{~min}$. The reaction container was cooled to $0{ }^{\circ} \mathrm{C}$ under argon atmosphere. To the solution MeOTf (1.5 equiv.) was added dropwise and sealed with insulating tape, followed by slow warming to room temperature with continued stirring for $12 \mathrm{~h}$. Next, DMSO ( $2 \mathrm{~mL}, 0.1 \mathrm{M})$ was added to the reaction at room temperature and purged with argon for $10 \mathrm{~min}$ to make the reaction mixture oxygen-free and sealed with electrical tape. The reaction mixture was shifted to $50{ }^{\circ} \mathrm{C}$ oil bath with continued stirring for $8 \mathrm{~h}$. The reaction mixture was quenched with saturated $\mathrm{NH}_{4} \mathrm{Cl}$ solution at room temperature and organic layer was extracted with EtOAc (3 times). The combined organic layer was dried over anhydrous $\mathrm{Na}_{2} \mathrm{SO}_{4}$. Solvent removal under reduced pressure gave the crude product which was further purified by column chromatography on silica gel using 1 - 2\% EtOAc-Pet ether as eluent to obtain the pure product.

The presence of oxygen led to oxidation by-products such as ester and thiazolone.

\section{G. Mechanistic Experiments:}

(a) Radical Trap Experiment<smiles>CC(OCc1ccccc1)c1nc2ccccc2s1</smiles>

4b

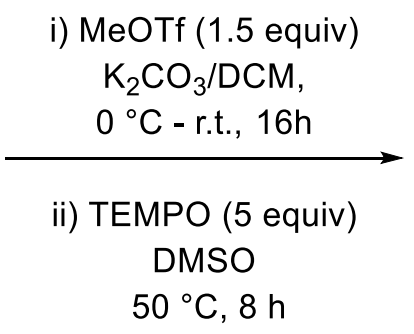

$50{ }^{\circ} \mathrm{C}, 8 \mathrm{~h}$

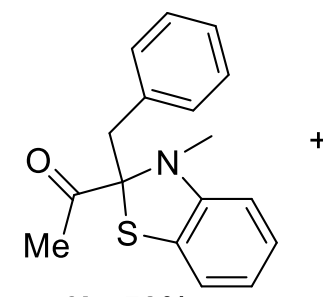

$6 b, 58 \%$<smiles>CC1(C)CCCC(C)(C)N1Oc1ccccc1</smiles>

$7,18 \%$

In a flame dried $25 \mathrm{ml}$ round bottom flask with a magnetic bar, ether $(0.2 \mathrm{mmol}, 1$ equiv. $)$ and $\mathrm{K}_{2} \mathrm{CO}_{3}(0.4 \mathrm{mmol}$, 2 equiv.) in dry DCM $(1 \mathrm{ml}, 0.2 \mathrm{M})$ were taken and sealed with electrical tape and degassed for $10 \mathrm{~min}$. The 
reaction container was cooled to $0{ }^{\circ} \mathrm{C}$ under argon atmosphere. To the solution MeOTf (1.5 equiv.) was added dropwise and sealed with insulating tape, followed by slow warming to room temperature with continued stirring for $12 \mathrm{~h}$. Next, DMSO ( $2 \mathrm{~mL}, 0.1 \mathrm{M})$ and TEMPO ( $1 \mathrm{mmol}, 5$ equiv.) was added to the reaction at room temperature and purged with argon for $10 \mathrm{~min}$ to make the reaction mixture oxygen-free and sealed with electrical tape. The reaction mixture was shifted to $50{ }^{\circ} \mathrm{C}$ oil bath with continued stirring for $8 \mathrm{~h}$. The reaction mixture was quenched with saturated $\mathrm{NH}_{4} \mathrm{Cl}$ solution at room temperature and organic layer was extracted with EtOAc (3 times). The combined organic layer was dried over anhydrous $\mathrm{Na}_{2} \mathrm{SO}_{4}$. Solvent removal under reduced pressure gave the crude product which was first analyzed by HRMS and ${ }^{1} \mathrm{H}-\mathrm{NMR}$, followed by further purifiecation via column chromatography on silica gel using 1.5 - 2\% EtOAc-Pet ether as eluent to rearranged product in 58\% yield and TEMPO-trapped benzyl compound (7) in $18 \%$ yield.

${ }^{1}$ H NMR (400 MHz, CDCl $) \delta 1.15(\mathrm{~s}, 6 \mathrm{H}), 1.26(\mathrm{~s}, 6 \mathrm{H}), 1.31-1.36(\mathrm{~m}, 1 \mathrm{H}), 1.45-1.56(\mathrm{~m}, 5 \mathrm{H}), 4.82(\mathrm{~s}, 2 \mathrm{H})$, 7.25 - 7.40 (m, 5H). ${ }^{13} \mathbf{C}$ NMR $\left(\mathbf{C D C l}_{3}, \mathbf{1 0 0} \mathbf{M H z}\right) \delta 17.1,20.3,33.1,39.7,60.0,78.7,127.3,127.4,128.2,138.3$. HRMS(ESI): $\mathrm{m} / \mathrm{z}$ calculated for $\mathrm{C}_{16} \mathrm{H}_{26} \mathrm{ON}[\mathrm{M}+\mathrm{H}]^{+}: 248.2009$, found: $248.2011 .{ }^{1} \mathbf{H}$ NMR (400 $\left.\mathbf{~ M H z}, \mathbf{C D C l}_{\mathbf{3}}\right) \delta$ $7.40-7.25(\mathrm{~m}, 5 \mathrm{H}), 4.82(\mathrm{~s}, 2 \mathrm{H}), 1.56-1.45(\mathrm{~m}, 5 \mathrm{H}), 1.36-1.31(\mathrm{~m}, 1 \mathrm{H}), 1.26(\mathrm{~s}, 6 \mathrm{H}), 1.15(\mathrm{~s}, 6 \mathrm{H}) .{ }^{13} \mathbf{C} \mathbf{N M R}$ $\left(\mathbf{C D C l}_{3}, \mathbf{1 0 0} \mathbf{M H z}\right) \delta 138.3,128.2,127.4,127.3,78.7,60.0,39.7,33.1,20.3,17.1$.

\section{(b) Results of Crossover Experiment:}<smiles>CC(OCc1ccccc1)c1nc2ccccc2s1</smiles><smiles>Clc1ccc(COC(CBr)C2CCCCC2)cc1</smiles>

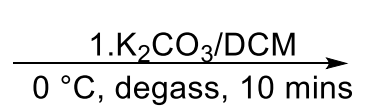
2. MeOTf, $0^{\circ} \mathrm{C}$ to $\mathrm{rt}, 12 \mathrm{~h}$ 3. DMSO, $50^{\circ} \mathrm{C}, 8 \mathrm{~h}$
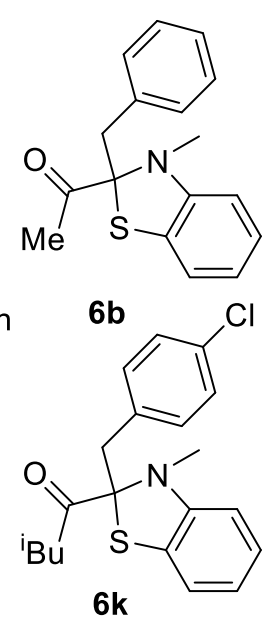

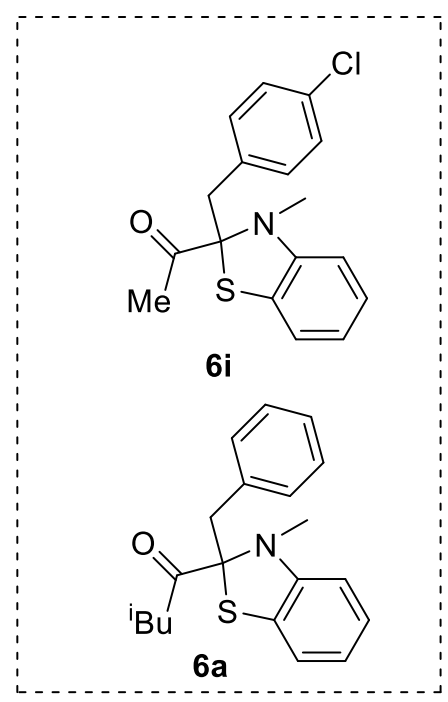

Observed by ${ }^{1} \mathrm{H}$ NMR, LCMS, HRMS

To a flame dried $25 \mathrm{ml}$ pear-shaped flask with a magnetic bar, $\mathbf{3 b}(0.1 \mathrm{mmol}), \mathbf{3 k}(0.1 \mathrm{mmol}), \mathrm{K}_{2} \mathrm{CO}_{3}(0.4 \mathrm{mmol})$ were taken and sealed the reaction flask with electrical tape. To the reaction flask, $1 \mathrm{ml}$ DCM $(0.2 \mathrm{M})$ was added and cooled to $0{ }^{\circ} \mathrm{C}$. MeOTf $(0.3 \mathrm{mmol})$ was added dropwise at $0{ }^{\circ} \mathrm{C}$ and slowly the resulting mixture allowed warm to room temperature with continued stirred for $12 \mathrm{~h}$. Next, DMSO $(2 \mathrm{ml}, 0.1 \mathrm{M})$ was added to the reaction at room temperature and purged with argon for $10 \mathrm{~min}$ to make the reaction mixture oxygen-free. The reaction flask was shifted at $50{ }^{\circ} \mathrm{C}$ oil bath with continued stirring for $8 \mathrm{~h}$. The reaction mixture was quenched with saturated $\mathrm{NH}_{4} \mathrm{Cl}$ solution at room temperature. The organic layer was extracted with EtOAc (3 times) and combined organic layer dried over anhydrous $\mathrm{Na}_{2} \mathrm{SO}_{4}$. Crude ${ }^{1} \mathrm{H}$ NMR and HRMS confirmed the normal and cross products. The ratio of normal and cross product was analyzed by HPLC $(\mathbf{6 b}: \mathbf{6 i}: \mathbf{6 k}: \mathbf{6 a}=31.63: 6.17$ : $61.68: 4.27)$ with YMC C-18 $(250 \mathrm{~mm} \times 4.6 \mathrm{~m}) 5$ micron column. Mobile Phase: $\mathrm{MeCN}: \mathrm{H}_{2} \mathrm{O}(80: 20)$. Wavelength: $254 \mathrm{~nm}$. Flow rate: $0.7 \mathrm{~mL} / \mathrm{min}$. 
Data Path: C: |WIN32APP \HSM $\backslash H P L C \backslash D A T A \backslash 0740 \backslash$

Processing Method: cal

System(acquisition): Sys 1

Series:0740

Application: HPLC

Volume: $10.0 \mathrm{ul}$

Sample Name: Four mix

Injection from this vial: 1 of 1

Sample Description: ACN:H2O(80:20)

Chrom Type: HPLC Channel : 1

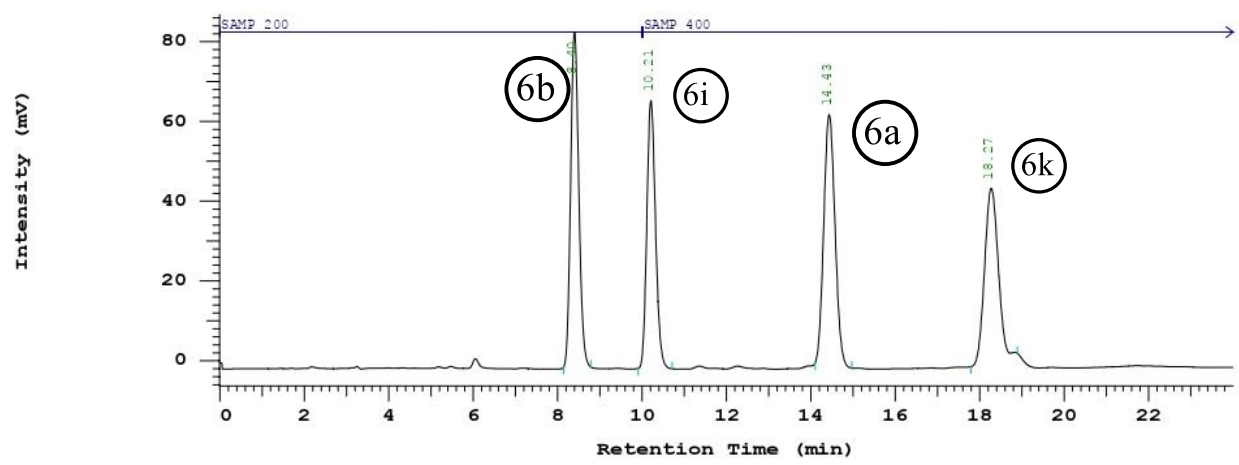

\begin{tabular}{|c|c|c|c|c|}
\hline No. & RT & Area & Cone 1 & $\mathrm{BC}$ \\
\hline 1 & 8.40 & 1093841 & 26.348 & $\mathrm{BB}$ \\
\hline 2 & 10.21 & 954533 & 22.993 & BB \\
\hline 3 & 14.43 & 1157156 & 27.874 & $\mathrm{BB}$ \\
\hline \multirow[t]{2}{*}{4} & 18.27 & 945921 & 22.785 & $\mathrm{BB}$ \\
\hline & & 4151451 & 100.000 & \\
\hline
\end{tabular}

Fig-1. HPLC traces for equimolar compound of $\mathbf{6 b}, \mathbf{6 i}, \mathbf{6} \mathbf{a}$ and $\mathbf{6 k}$.

Data Path: C: IWIN32APP $\backslash H S M \backslash H P L C \backslash D A T A \backslash 0741 \_001 \backslash$

Processing Method: cal

System(acquisition): Sys 1

Series:0741 001

Application: HPLC

Volume: $10.0 \mathrm{ul}$

Sample Name: Four mix

Injection from this vial: 1 of 1

Sample Description: ACN:H2O(80:20)

Chrom Type: HPLC Channel : 1

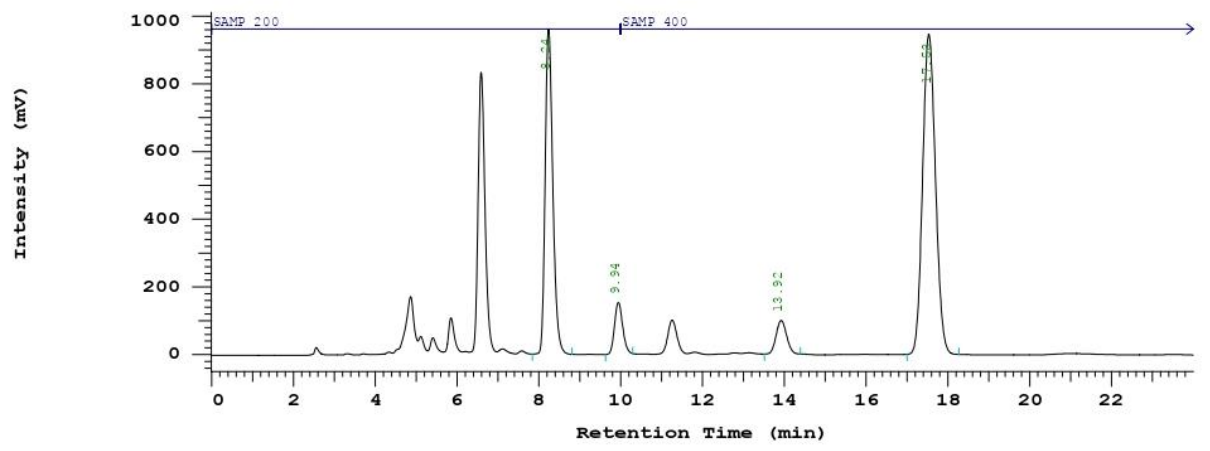

\begin{tabular}{|c|c|c|c|c|}
\hline No. & $\mathrm{RT}$ & Area & Cone 1 & $\mathrm{BC}$ \\
\hline 1 & 8.24 & 12216673 & 33.333 & BB \\
\hline 2 & 9.94 & 2085005 & 5.689 & BB \\
\hline 3 & 13.92 & 1746643 & 4.766 & $\mathrm{BB}$ \\
\hline 4 & 17.53 & 20601550 & 56.212 & BB \\
\hline & & 36649871 & 100.000 & \\
\hline
\end{tabular}

Fig-2. HPLC traces of the reaction crude from crossover experiments 


\section{(c) Kinetics Studies:}

The formation of ether intermediates (5), rate of formation of products (6) and rate of disapappearances of 5 were monitored by ${ }^{1} \mathrm{H}$ NMR on a Bruker Avance $400 \mathrm{MHz}$ spectrometer with temperature control probe. An equimolar mixture of $\mathrm{N}$-methylated salts of both $\mathbf{4 b}(0.025 \mathrm{mmol})$ and $4 \mathrm{~s}(0.025 \mathrm{mmol})$ in DMSO- $d_{6}(0.5 \mathrm{~mL}$, $0.05 \mathrm{M}$ each) was taken in a NMR tube with 1,4-dimethoxybenzene $(0.05 \mathrm{mmol})$ as an internal standard and treated with soluble base DBU at room temperature. A rapid consumption of the $\mathrm{N}$-methylated salt to the diastereomeric precursors $\mathbf{5 b}, \mathbf{5} \mathbf{b}^{\prime}$ and $\mathbf{5 s}, \mathbf{5} \mathbf{s}^{\prime}$ at room temperature was checked via ${ }^{1} \mathrm{H}$ NMR. Then the NMR tube was taken out from the instrument and NMR probe was heated to $40{ }^{\circ} \mathrm{C}$. The NMR tube with intermediates was reloaded in the probe for its kinetic studiy. The thermal rearrangement was followed via recording ${ }^{1} \mathrm{H}$ NMR spectra at a regular time interval at $40{ }^{\circ} \mathrm{C}$.

Average N-Me peak areas of the products $\mathbf{6 b}$, and $\mathbf{6 s}$ were integrated and compared with that of the internal standard for their rate of formations.

Also, average benzylic protons peak areas of the precursors $\mathbf{5 b}, \mathbf{5} \mathbf{b}^{\prime}$ and $\mathbf{5} \mathbf{s}, \mathbf{5} \mathbf{s}^{\prime}$ were integrated and compared with that of an internal standard for their rate of disappearances.

The 1st order kinetics for both the substrate studied. Plots for disappearances of $\mathbf{5}$ versus time were found to decrease linerarly $\left(\mathrm{R}^{2}=0.991\right.$ for $\mathbf{4 b}$ and 0.957 for $\mathbf{4 s}$ substrates) over a significant range of conversion and rate constant were calculated via plotting $\ln [5]$ vs time in second. Study showed that 4-cyano substitution accelerated the rearrangement 3.76 times Table S2), further supporting the radical pathway. The ratio of product formation at $\sim 50 \%$ conversion was also calculated to be $3.3: 1$ by ${ }^{1} \mathrm{H}$ NMR.

Table S1. The kinetic study of thermal [1,3] rearrangement of the N-methylated salt (consumption of starting materials)
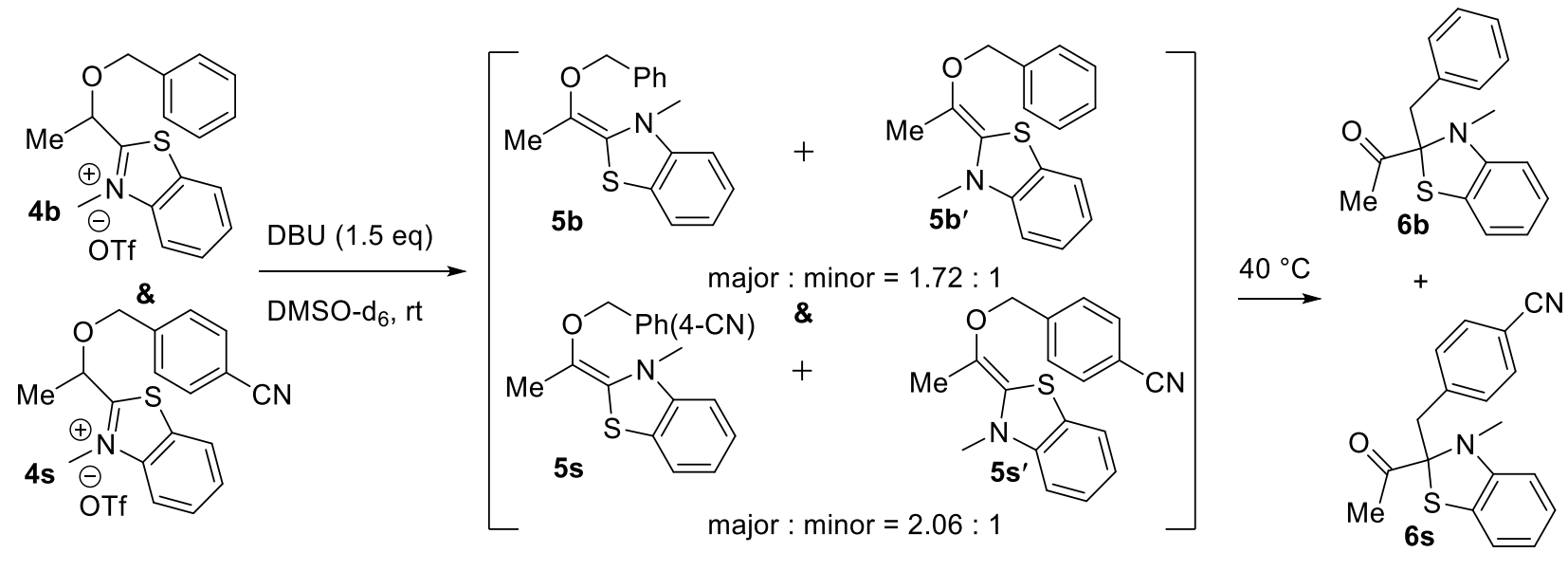

\begin{tabular}{cccccccc}
\hline Entry & * NMR value & ${ }^{* *} \mathrm{p}-\mathrm{H} \times 0.1$ & ${ }^{*} \mathrm{NMR}$ value & ${ }^{* *} \mathrm{p}-\mathrm{CN} \times 0.1$ & & & \\
& $\mathrm{p}-\mathrm{H}$ & {$[\mathrm{M}]$} & $\mathrm{p}-\mathrm{CN}$ & {$[\mathrm{M}]$} & time $(\mathrm{sec})$ & $\ln [\mathrm{p}-\mathrm{H}]$ & $\ln [\mathrm{p}-\mathrm{CN}]$ \\
$*_{* * *} 1$ & 0.49 & 0.049 & 0.445 & 0.0445 & 0 & -3.016 & -3.112 \\
2 & 0.475 & 0.0475 & 0.34 & 0.034 & 240 & -3.047 & -3.381 \\
3 & 0.45 & 0.045 & 0.28 & 0.028 & 480 & -3.101 & -3.575 \\
4 & 0.44 & 0.044 & 0.24 & 0.024 & 720 & -3.123 & -3.73 \\
5 & 0.42 & 0.042 & 0.195 & 0.0195 & 960 & -3.17 & -3.937 \\
6 & 0.415 & 0.0415 & 0.18 & 0.018 & 1200 & -3.182 & -4.017 \\
7 & 0.37 & 0.037 & 0.135 & 0.0135 & 1920 & -3.296 & -4.305 \\
8 & 0.345 & 0.0345 & 0.105 & 0.0105 & 2640 & -3.366 & -4.556 \\
9 & 0.32 & 0.032 & 0.08 & 0.008 & 3360 & -3.442 & -4.828
\end{tabular}

\footnotetext{
* Sum of $\left(\mathbf{5} \mathbf{a}+\mathbf{5} \mathbf{a}^{\prime}\right)$ and $\left(\mathbf{5} \mathbf{s}+\mathbf{5} \mathbf{s}^{\prime}\right)$ were taken.

$* *$ NMR value $\times 0.1$ (concentration of internal standard).

**** only traces of the products were detected.
} 
Table S2. Rate comparisn (consumption of starting materials).

\begin{tabular}{cccc} 
Substrate & *rate $(\mathrm{M} / \mathrm{s})$ & $\mathrm{k}(1 / \mathrm{s})$ & $\mathrm{k}_{\mathrm{CN}} / \mathrm{k}_{\mathrm{H}}$ \\
& & & 3.7 \\
$4-\mathrm{CN}(\mathbf{4 s})$ & 0.00004788 & 0.0004788 & \\
$4-\mathrm{H}(\mathbf{4 a})$ & 0.00001272 & 0.0001272 & \\
\hline
\end{tabular}

*rate constant value multiply by concentration of the solution (0.1M Internal standard)

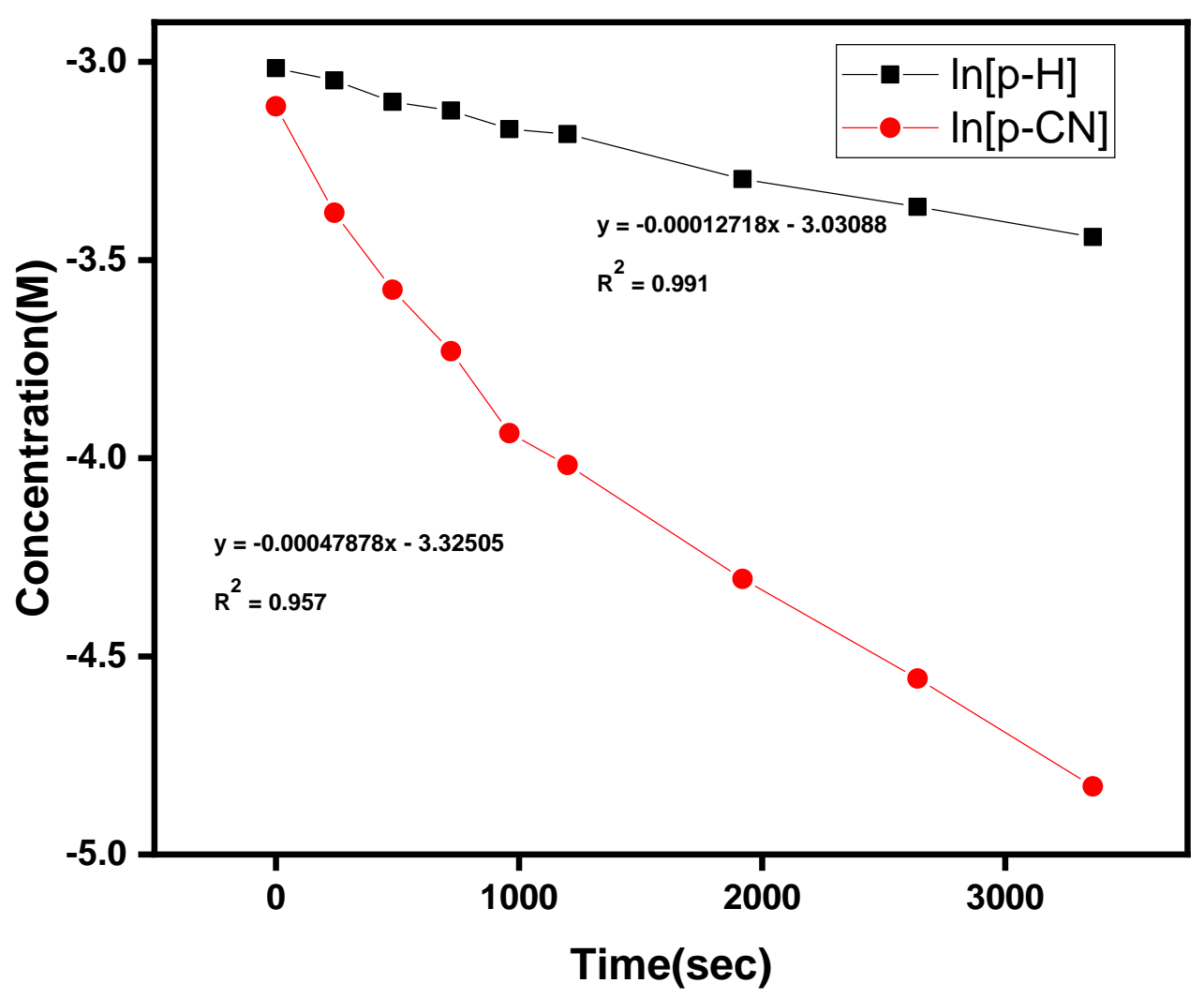

Fig-3. Plot data with concentration Vs time

\section{${ }^{1} \mathrm{H}$ NMR analysis at $\sim \mathbf{5 0 \%}$ conversion:}

The peak around $3.69(6 \mathrm{H}) \mathrm{ppm}$ is correspond to internal standard (1, 4-dimethoxybenzene) whereas the peak 4.67, $4.72(2 \mathrm{H}) \mathrm{ppm}$ are for the $\mathbf{5} \mathbf{b}, \mathbf{5} \mathbf{b}^{\prime}$ and $4.77,4.82(2 \mathrm{H}) \mathrm{ppm}$ for the $\mathbf{5} \mathbf{s}, \mathbf{5} \mathbf{s}^{\prime}$ isomeric precursors respectively. Average peak area of $\mathbf{5} \mathbf{b}, \mathbf{5} \mathbf{b}^{\prime}$ and $\mathbf{5} \mathbf{s}, \mathbf{5} \mathbf{s}^{\prime}$ were measured by integrating areas of each of the peaks compared shown in red with that of an internal standard (Table S1, column-2 \& 4). To get the approximate concentration of the precursors, the average value was multiplying by the internal standard concentration $(\mathbf{0 . 1 M})$ (Table S1, column-3 \& 5). 
<smiles>CC(OC([14CH3])c1ccc(C#N)cc1)=C1Sc2ccccc2N1C</smiles>

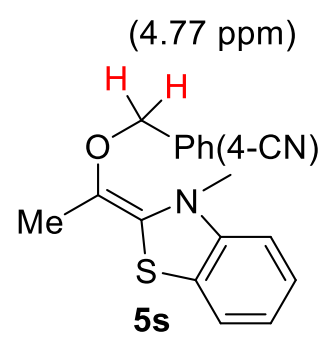<smiles>CC(=O)OC([14CH3])([14CH3])c1ccccc1</smiles><smiles>Cc1c(OC(c2ccccc2)C([18OH])[PH2+])c2ccccc2n1C</smiles>

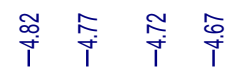

Internał standard

(5s) $5 s^{\prime} 5 a$

precursor ratio $\mathbf{5 b}, \mathbf{5} \mathbf{b}^{\prime}, \mathbf{5} \mathbf{s}$ and $\mathbf{5} \mathbf{s}^{\prime}$ at $25^{\circ} \mathrm{C}, \mathrm{t}=0 \mathrm{sec}$

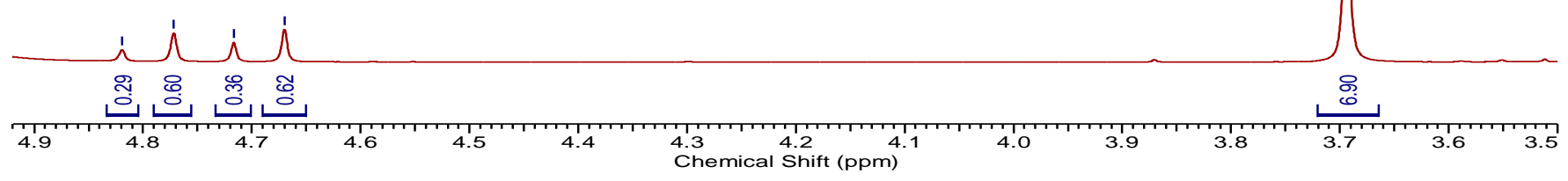

Internal standard

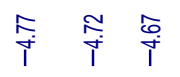

precursor ratio $\mathbf{5 b}, \mathbf{5} \mathbf{b}^{\prime}$ and $\mathbf{5 s}$ at $40^{\circ} \mathrm{C}, \mathrm{t}=960 \mathrm{sec}$

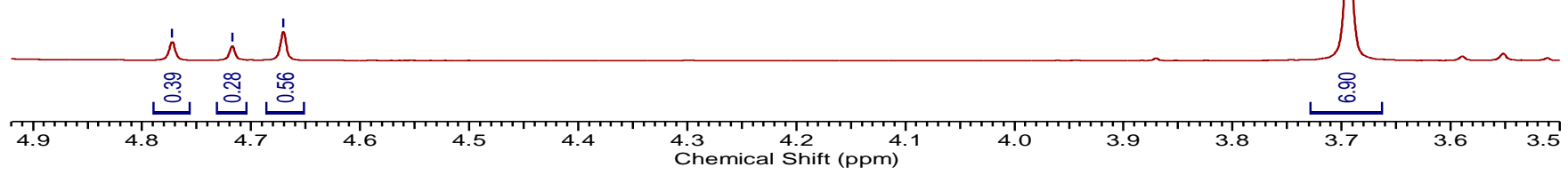



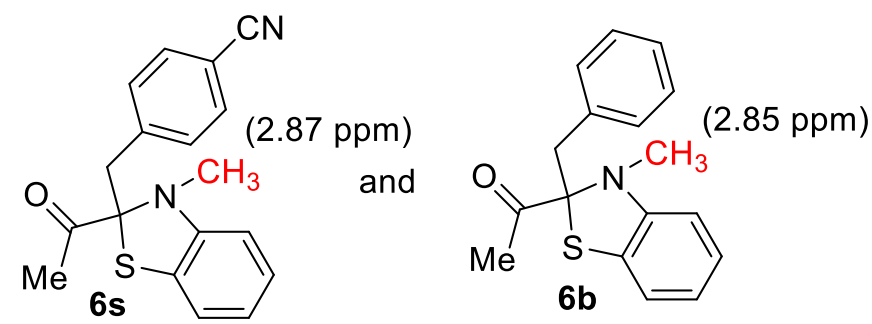

Internal standard

competitive product formation $6 \mathrm{~b}$ and $6 \mathrm{~s}$ at $40^{\circ} \mathrm{C}, \mathrm{t}=960 \mathrm{sec}$

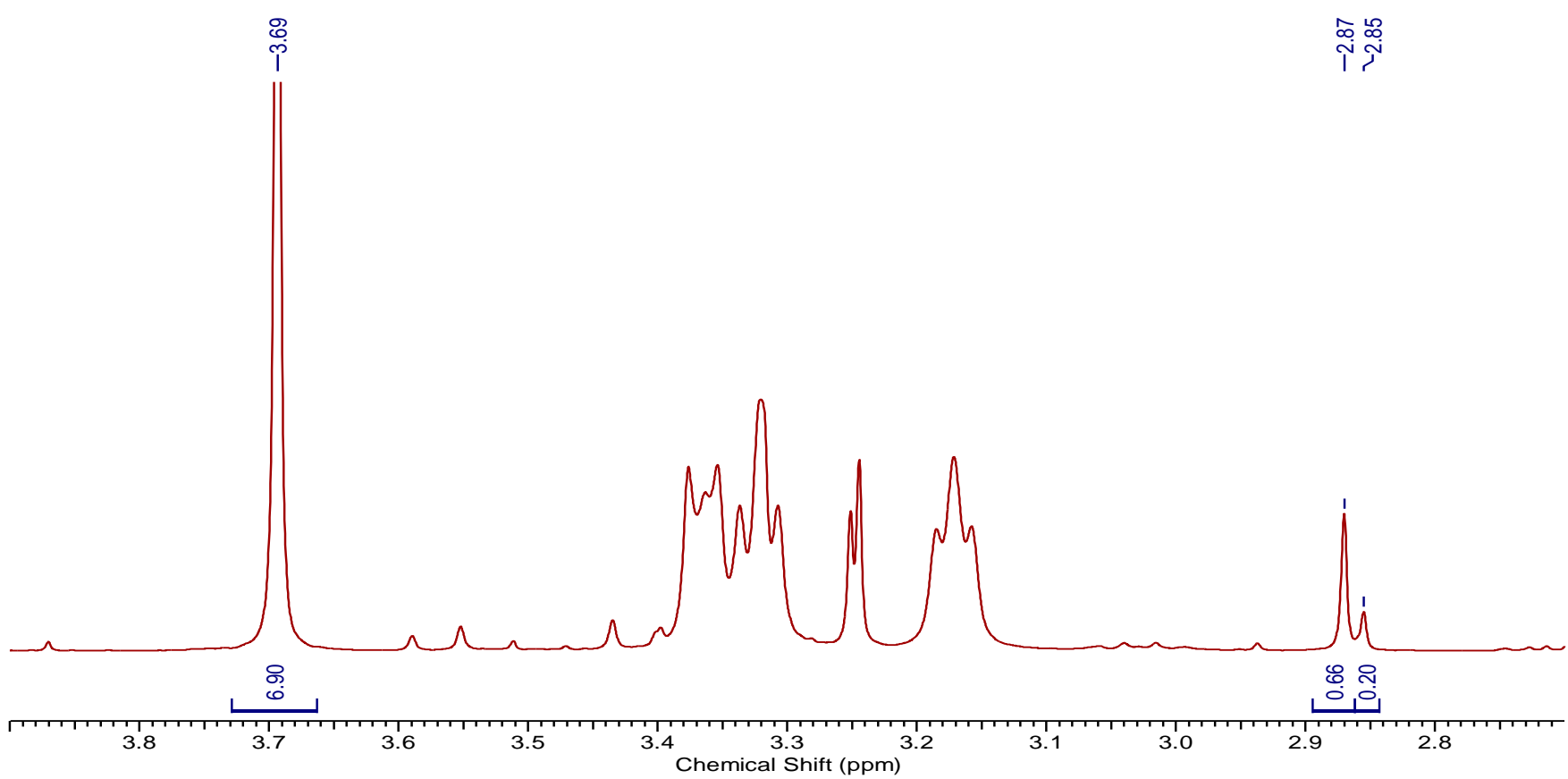

Product formation ratio $\sim 50 \%$ conversion: $\mathbf{6 s : 6 b}=3.3$

Table S3. Comparison of the rate of formation of $[1,3]$ product in DMSO- $\mathrm{d}_{6}$ and Toluene- $\mathrm{d}_{8}$.

\begin{tabular}{|l|l|}
\hline Entry & {$[1,3]$ product } \\
\hline DMSO-d & $37-38 \%$ \\
\hline Toluene-d $_{8}$ & $23-24 \%$ \\
\hline
\end{tabular}




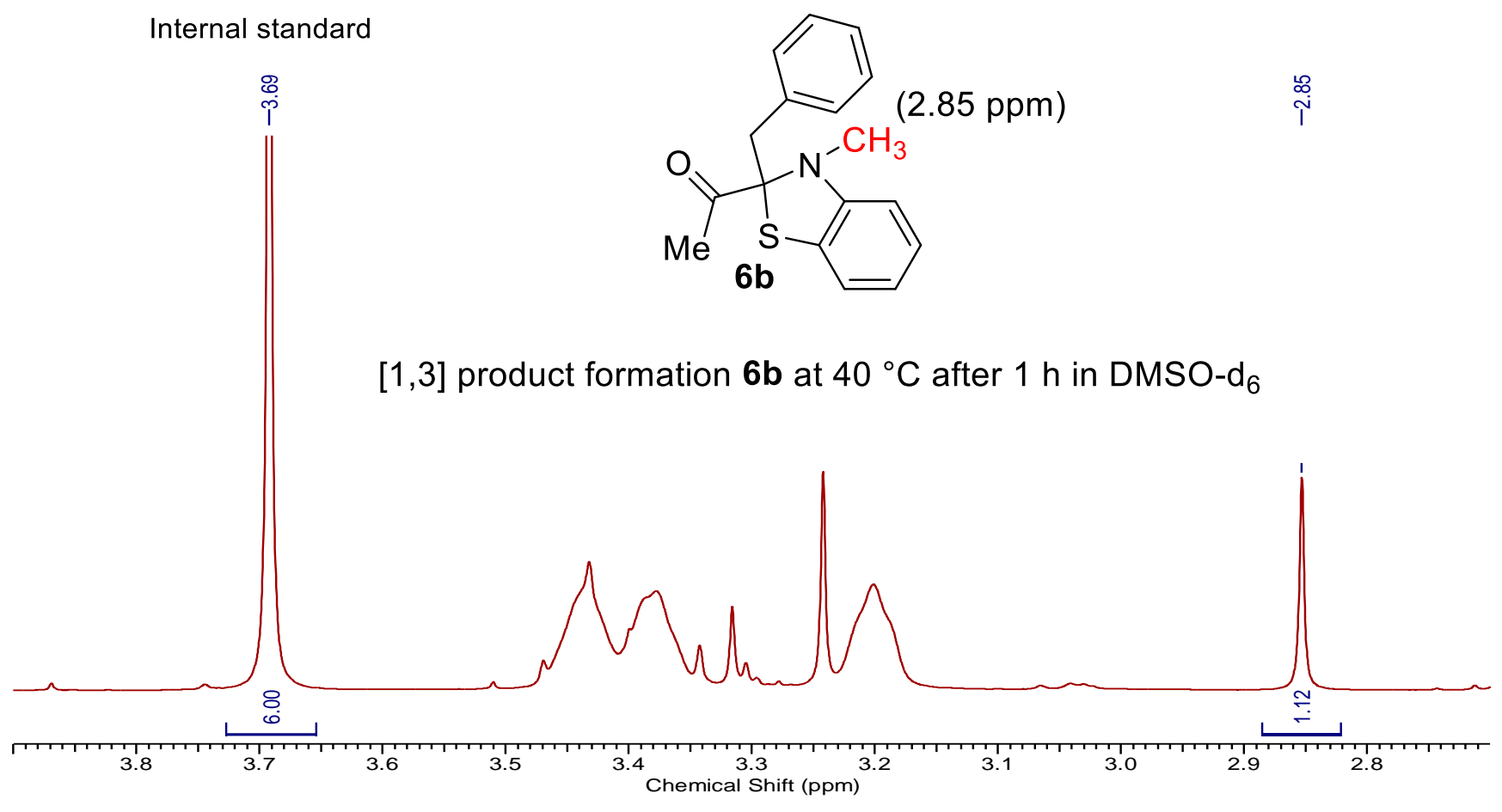

Internal standard

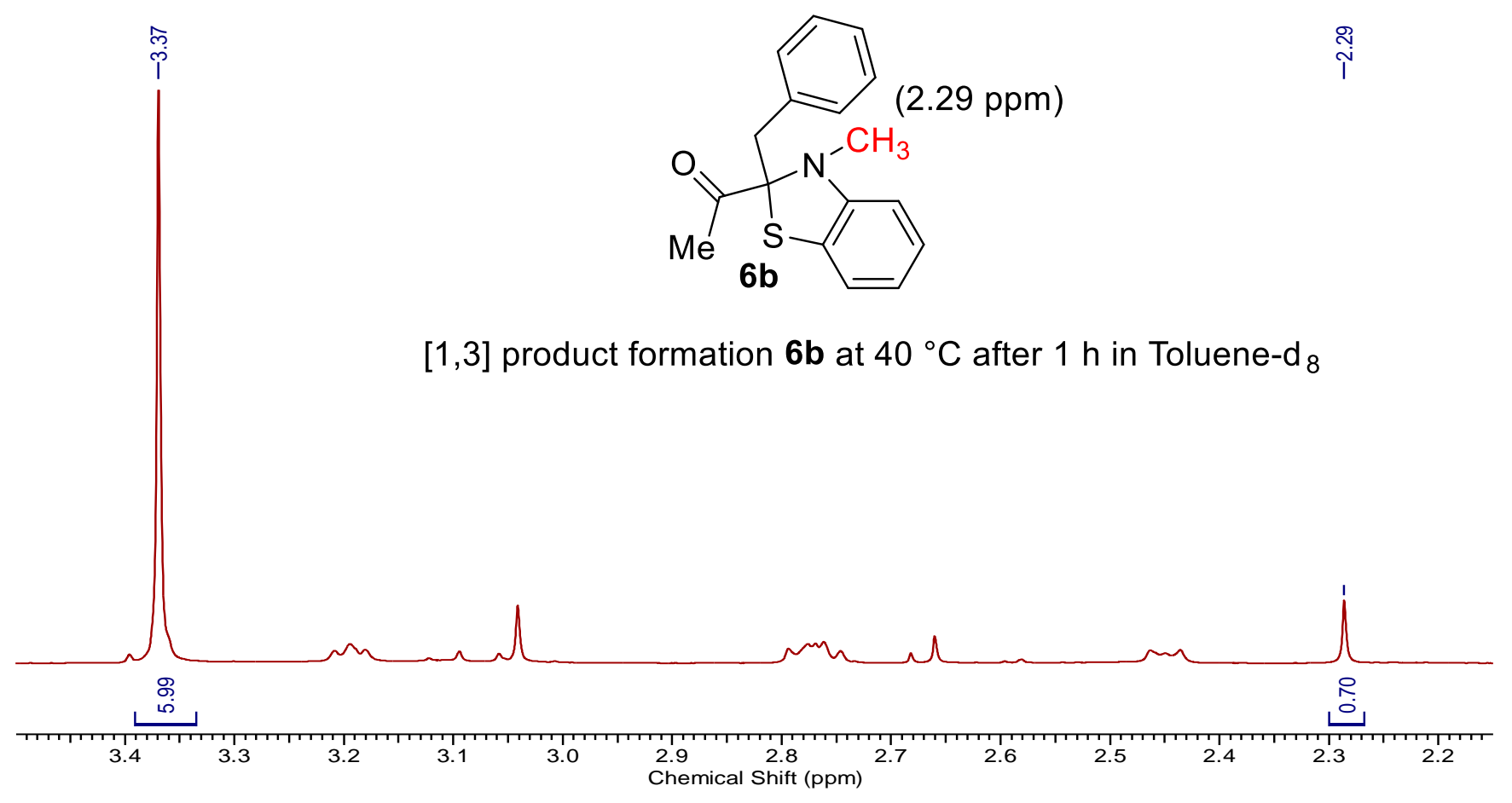




\section{H. Functionalization of $[1,3]$ products:}

\section{(a) Deprotection: ${ }^{7}$}<smiles>[R]C(=O)C(=O)Cc1ccc([R])cc1</smiles>

In an oven dried $25 \mathrm{ml}$ pear-shaped flask with a magnetic bar, $6(0.1 \mathrm{mmol})$ was taken in $\mathrm{CH}_{3} \mathrm{CN}(1.5 \mathrm{ml}, 0.7 \mathrm{M})$ at room temperature under argon atmosphere. To the reaction flask $\mathrm{AgBF}_{4}$ (3 equiv.) in $0.25 \mathrm{ml}_{2} \mathrm{O}$ was added and the resulting solution was stirred for $10 \mathrm{~min}$ at room temperature. Then the reaction flask was stirred with heating in an oil bath at $60{ }^{\circ} \mathrm{C}$ for $2 \mathrm{~h}$. Saturated aq. $\mathrm{NaHCO}_{3}$ solution was added to quench the reaction and filtered though celite.The organic layer was extracted with EtOAc (3 times) and combined organic layer dried over anhydrous $\mathrm{Na}_{2} \mathrm{SO}_{4}$. The solvent removal under reduced pressure gave the crude product which was further purified by column chromatography on silica gel using $1-1.5 \%$ as eluent to obtain the desired product.

(b) Alpha-Hydroxy Ketone: ${ }^{10}$
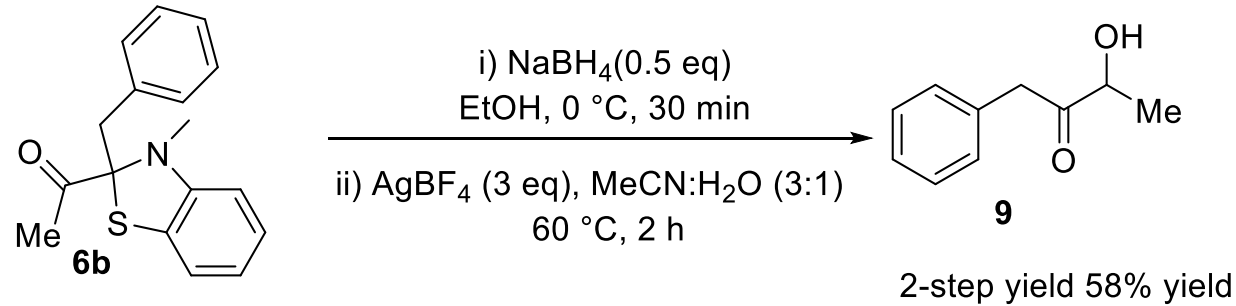

1st step: In an oven dried $25 \mathrm{ml}$ pear-shaped flask with a magnetic bar, $\mathbf{6 b}(0.173 \mathrm{mmol})$ was taken in EtOH $(1.5$ $\mathrm{ml}, 0.12 \mathrm{M})$ under argon atmosphere and cooled to $0{ }^{\circ} \mathrm{C}$. To the reaction mixture $\mathrm{NaBH}_{4}(0.5$ equiv. $)$ was added portion wise and stirred for $30 \mathrm{~min}$. Saturated aq. $\mathrm{NH}_{4} \mathrm{Cl}$ was added to quench the reaction at $0{ }^{\circ} \mathrm{C}$. The organic layer was extracted with EtOAc (3 times) and combined organic layer dried over anhydrous $\mathrm{Na}_{2} \mathrm{SO}_{4}$. The solvent removal under reduced pressure gave the crude product which was used for next step without further purification.

(c) Wittig Olefination: ${ }^{12}$

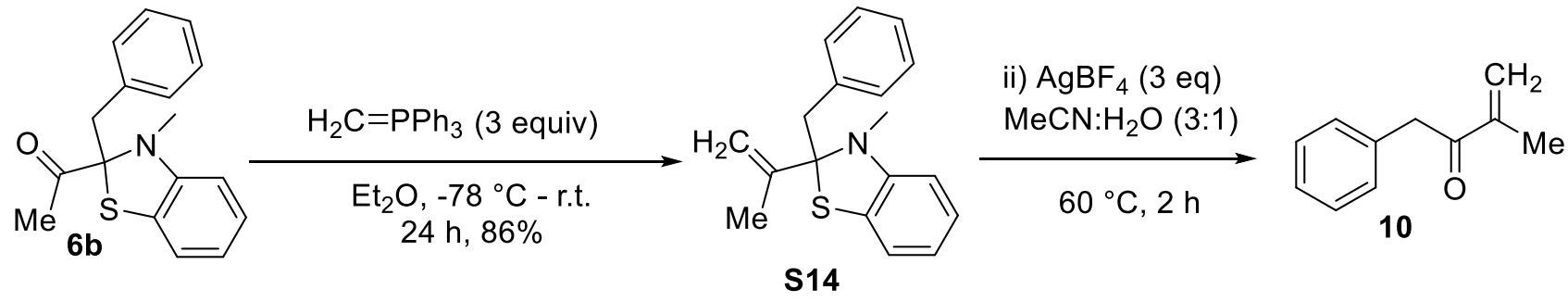

$1^{\text {st }}$ step: In an oven dried $25 \mathrm{ml}$ pear-shaped flask with a magnetic bar, methyl triphenylphosphonium bromide $(0.75 \mathrm{mmol}, 3 \mathrm{eq})$ was taken in $\operatorname{dry~}_{\mathrm{Et}} \mathrm{O}(2 \mathrm{ml})$ under argon atmosphere and cooled to $0{ }^{\circ} \mathrm{C}$. $\mathrm{n}$ - $\mathrm{BuLi}(0.75 \mathrm{mmol}$, 3 equiv.) was added dropwise and the resulting solution was stirred for $20 \mathrm{~min}$. The reaction mixture was cooled

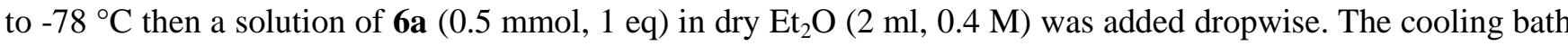
was removed and the reaction mixture was slowly brought to room temperature and stirred for $24 \mathrm{~h} . \mathrm{NH}_{4} \mathrm{Cl}$ was added to quench the reaction and extracted with $\mathrm{Et}_{2} \mathrm{O}$ ( 3 times) and combined organic layer dried over anhydrous 
$\mathrm{Na}_{2} \mathrm{SO}_{4}$. The solvent removal under reduced pressure gave the crude product which was purified by silica gel column chromatography using $1 \%$ EtOAc-Pet ether as eluent to obatined in $86 \%$ yield $(\mathbf{S 1 4}, 61 \mathrm{mg})$.

The second step was performed as above, leading to $69 \%$ of $\mathbf{1 0 .}$

\section{Computational Method:}

All the DFT calculations were carried out by using Gaussian 09 program ${ }^{14}$ with the (U) $\omega$ B97XD functional ${ }^{15}$. The $6-31+\mathrm{G}(\mathrm{d}, \mathrm{p})$ basis set ${ }^{16}$ was employed for all the atoms. Universal solvation model (SMD) ${ }^{17}$ was used for all solvent phase calculations. Harmonic frequency calculations were performed for all stationary points to confirm them as minima. Thermal correction and zero point vibration energies were incorporated in the $\Delta \mathrm{G}$ value.

\section{A. Optimized Cartesian Coordinates:}

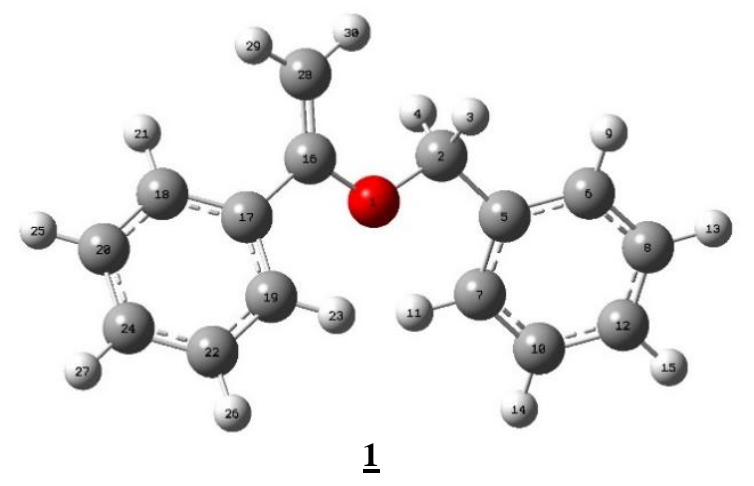

\begin{tabular}{|c|c|c|c|c|c|c|c|c|c|c|c|}
\hline \multicolumn{4}{|c|}{1 (Gas) } & \multicolumn{4}{|c|}{1 (DMSO) } & \multicolumn{4}{|c|}{1 (Toluene) } \\
\hline $\mathrm{O}$ & 0.031995 & 0.590378 & -0.020401 & $\mathrm{O}$ & -0.032495 & 0.570241 & 0.019781 & $\mathrm{O}$ & 0.029766 & 0.577979 & -0.013118 \\
\hline $\mathrm{C}$ & 1.221095 & 1.352634 & 0.017823 & $\mathrm{C}$ & -1.229536 & 1.332191 & 0.007186 & $\mathrm{C}$ & 1.220621 & 1.340735 & 0.041734 \\
\hline $\mathrm{H}$ & 1.244075 & 1.964646 & 0.930040 & $\mathrm{H}$ & -1.262864 & 1.961893 & -0.890791 & $\mathrm{H}$ & 1.240614 & 1.933735 & 0.966035 \\
\hline $\mathrm{H}$ & 1.243110 & 2.033488 & -0.846135 & $\mathrm{H}$ & -1.249089 & 1.994130 & 0.884276 & $\mathrm{H}$ & 1.245807 & 2.038325 & -0.808021 \\
\hline $\mathrm{C}$ & 2.405527 & 0.420648 & -0.022234 & $\mathrm{C}$ & -2.411544 & 0.397464 & 0.036433 & $\mathrm{C}$ & 2.408190 & 0.413209 & -0.013652 \\
\hline C & 3.611141 & 0.813719 & 0.561091 & C & -3.626977 & 0.820996 & -0.508603 & C & 3.621254 & 0.823267 & 0.544300 \\
\hline $\mathrm{C}$ & 2.331625 & -0.814535 & -0.668617 & C & -2.336823 & -0.862468 & 0.636032 & C & 2.336208 & -0.826991 & -0.651328 \\
\hline $\mathrm{C}$ & 4.731864 & -0.010809 & 0.495633 & $\mathrm{C}$ & -4.752955 & 0.001272 & -0.452727 & $\mathrm{C}$ & 4.748337 & 0.008323 & 0.466171 \\
\hline $\mathrm{H}$ & 3.675883 & 1.769167 & 1.076067 & $\mathrm{H}$ & -3.692785 & 1.797129 & -0.982875 & $\mathrm{H}$ & 3.685269 & 1.783991 & 1.049592 \\
\hline $\mathrm{C}$ & 3.450007 & -1.642674 & -0.727477 & $\mathrm{C}$ & -3.461183 & -1.686190 & 0.684773 & C & 3.461885 & -1.645618 & -0.722830 \\
\hline $\mathrm{H}$ & 1.393126 & -1.127378 & -1.113865 & $\mathrm{H}$ & -1.397485 & -1.204130 & 1.058828 & $\mathrm{H}$ & 1.395423 & -1.154499 & -1.081323 \\
\hline $\mathrm{C}$ & 4.653084 & -1.243278 & -0.148901 & $\mathrm{C}$ & -4.672376 & -1.257177 & 0.143307 & $\mathrm{C}$ & 4.670963 & -1.230247 & -0.168231 \\
\hline $\mathrm{H}$ & 5.662321 & 0.306158 & 0.956267 & $\mathrm{H}$ & -5.689847 & 0.343428 & -0.882372 & $\mathrm{H}$ & 5.683777 & 0.338638 & 0.907531 \\
\hline $\mathrm{H}$ & 3.379856 & -2.603579 & -1.227880 & $\mathrm{H}$ & -3.388515 & -2.665290 & 1.149278 & $\mathrm{H}$ & 3.392591 & -2.610338 & -1.216476 \\
\hline $\mathrm{H}$ & 5.522584 & -1.891562 & -0.195180 & $\mathrm{H}$ & -5.546403 & -1.900674 & 0.181698 & $\mathrm{H}$ & 5.546149 & -1.870416 & -0.224678 \\
\hline $\mathrm{C}$ & -1.156193 & 1.246509 & 0.022726 & $\mathrm{C}$ & 1.148109 & 1.238445 & -0.011634 & $\mathrm{C}$ & -1.155928 & 1.236908 & 0.029265 \\
\hline $\mathrm{C}$ & -2.287436 & 0.283678 & 0.033502 & C & 2.293257 & 0.289692 & -0.044373 & C & -2.292850 & 0.279241 & 0.033684 \\
\hline $\mathrm{C}$ & -3.526894 & 0.620721 & -0.520490 & $\mathrm{C}$ & 3.526751 & 0.630445 & 0.524445 & C & -3.523140 & 0.618453 & -0.540441 \\
\hline $\mathrm{C}$ & -2.121547 & -0.984874 & 0.600401 & $\mathrm{C}$ & 2.149363 & -0.965720 & -0.648618 & C & -2.142731 & -0.984431 & 0.616916 \\
\hline $\mathrm{C}$ & -4.584501 & -0.282693 & -0.488847 & $\mathrm{C}$ & 4.597379 & -0.258609 & 0.474509 & $\mathrm{C}$ & -4.586633 & -0.278967 & -0.514269 \\
\hline $\mathrm{H}$ & -3.658517 & 1.586157 & -0.998701 & $\mathrm{H}$ & 3.648039 & 1.587024 & 1.023724 & $\mathrm{H}$ & -3.643814 & 1.580992 & -1.027969 \\
\hline $\mathrm{C}$ & -3.180405 & -1.887409 & 0.630731 & $\mathrm{C}$ & 3.221600 & -1.853618 & -0.697199 & $\mathrm{C}$ & -3.207526 & -1.880697 & 0.641642 \\
\hline $\mathrm{H}$ & -1.159395 & -1.255916 & 1.020926 & $\mathrm{H}$ & 1.197025 & -1.243640 & -1.087674 & $\mathrm{H}$ & -1.189483 & -1.259077 & 1.055588 \\
\hline $\mathrm{C}$ & -4.415771 & -1.539575 & 0.088705 & $\mathrm{C}$ & 4.449411 & -1.503309 & -0.137136 & $\mathrm{C}$ & -4.433256 & -1.531235 & 0.078386 \\
\hline $\mathrm{H}$ & -5.538293 & -0.009311 & -0.929234 & $\mathrm{H}$ & 5.545575 & 0.017238 & 0.926153 & $\mathrm{H}$ & -5.532803 & -0.004181 & -0.970481 \\
\hline $\mathrm{H}$ & -3.039194 & -2.865458 & 1.080150 & $\mathrm{H}$ & 3.095992 & -2.820679 & -1.175023 & $\mathrm{H}$ & -3.078175 & -2.854978 & 1.103140 \\
\hline $\mathrm{H}$ & -5.239149 & -2.246702 & 0.108444 & $\mathrm{H}$ & 5.282928 & -2.198562 & -0.170586 & $\mathrm{H}$ & -5.260740 & -2.234080 & 0.093037 \\
\hline $\mathrm{C}$ & -1.299599 & 2.579304 & 0.072440 & C & 1.280116 & 2.574255 & -0.031270 & C & -1.296597 & 2.570142 & 0.084186 \\
\hline $\mathrm{H}$ & -2.289493 & 3.007590 & 0.157531 & $\mathrm{H}$ & 2.268844 & 3.012174 & -0.098248 & $\mathrm{H}$ & -2.286718 & 3.000545 & 0.165834 \\
\hline $\mathrm{H}$ & -0.464282 & 3.266828 & 0.053308 & $\mathrm{H}$ & 0.436802 & 3.253413 & -0.000644 & $\mathrm{H}$ & -0.459833 & 3.257145 & 0.073597 \\
\hline
\end{tabular}




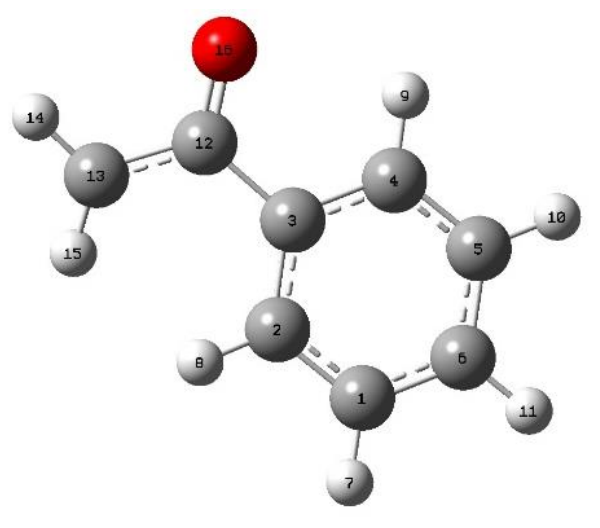

I

\begin{tabular}{|c|c|c|c|c|c|c|c|c|c|c|c|}
\hline \multicolumn{4}{|c|}{ I (Gas) } & \multicolumn{4}{|c|}{ I (DMSO) } & \multicolumn{4}{|c|}{ I (Toluene) } \\
\hline $\mathrm{C}$ & 1.831154 & 1.234297 & -0.102468 & $\mathrm{C}$ & 1.824466 & 1.232168 & -0.136608 & $\mathrm{C}$ & 1.826940 & 1.233890 & -0.124049 \\
\hline $\mathrm{C}$ & 0.439075 & 1.207378 & -0.122331 & $\mathrm{C}$ & 0.431508 & 1.202059 & -0.157252 & $\mathrm{C}$ & 0.434879 & 1.204889 & -0.153895 \\
\hline $\mathrm{C}$ & -0.246320 & -0.007855 & -0.025494 & $\mathrm{C}$ & -0.249518 & -0.014554 & -0.030234 & $\mathrm{C}$ & -0.248909 & -0.010647 & -0.042465 \\
\hline $\mathrm{C}$ & 0.483263 & -1.196008 & 0.085211 & $\mathrm{C}$ & 0.484309 & -1.197744 & 0.117332 & $\mathrm{C}$ & 0.481438 & -1.195930 & 0.095434 \\
\hline $\mathrm{C}$ & 1.872787 & -1.168069 & 0.115290 & $\mathrm{C}$ & 1.874867 & -1.164502 & 0.151731 & $\mathrm{C}$ & 1.870948 & -1.164623 & 0.139323 \\
\hline $\mathrm{C}$ & 2.549617 & 0.047564 & 0.021632 & C & 2.547803 & 0.051240 & 0.022970 & $\mathrm{C}$ & 2.546301 & 0.050634 & 0.029004 \\
\hline $\mathrm{H}$ & 2.353010 & 2.182016 & -0.188179 & $\mathrm{H}$ & 2.343289 & 2.179378 & -0.247319 & $\mathrm{H}$ & 2.348313 & 2.180755 & -0.223845 \\
\hline $\mathrm{H}$ & -0.101931 & 2.141408 & -0.236456 & $\mathrm{H}$ & -0.114128 & 2.130611 & -0.293807 & $\mathrm{H}$ & -0.109299 & 2.134626 & -0.289630 \\
\hline $\mathrm{H}$ & -0.058063 & -2.134159 & 0.146679 & $\mathrm{H}$ & -0.042816 & -2.141756 & 0.212730 & $\mathrm{H}$ & -0.052253 & -2.137626 & 0.171625 \\
\hline $\mathrm{H}$ & 2.428702 & -2.095456 & 0.209702 & $\mathrm{H}$ & 2.433111 & -2.087108 & 0.278427 & $\mathrm{H}$ & 2.427181 & -2.089309 & 0.257862 \\
\hline $\mathrm{H}$ & 3.634830 & 0.069667 & 0.042021 & $\mathrm{H}$ & 3.633266 & 0.077582 & 0.045812 & $\mathrm{H}$ & 3.631493 & 0.074861 & 0.058902 \\
\hline $\mathrm{C}$ & -1.744431 & -0.098419 & -0.057451 & $\mathrm{C}$ & -1.746609 & -0.094917 & -0.071677 & $\mathrm{C}$ & -1.745984 & -0.092059 & -0.092261 \\
\hline $\mathrm{C}$ & -2.551548 & 1.062300 & 0.210944 & $\mathrm{C}$ & -2.537339 & 1.048241 & 0.283170 & $\mathrm{C}$ & -2.542301 & 1.054458 & 0.248243 \\
\hline $\mathrm{H}$ & -3.627088 & 0.950480 & 0.137360 & $\mathrm{H}$ & -3.616635 & 0.965588 & 0.208305 & $\mathrm{H}$ & -3.619709 & 0.967052 & 0.159004 \\
\hline $\mathrm{H}$ & -2.139451 & 2.014507 & 0.523790 & $\mathrm{H}$ & -2.106079 & 1.968381 & 0.660690 & $\mathrm{H}$ & -2.119398 & 1.973662 & 0.637678 \\
\hline $\mathrm{O}$ & -2.299436 & -1.181673 & -0.285288 & $\mathrm{O}$ & -2.306826 & -1.160011 & -0.385366 & $\mathrm{O}$ & -2.305469 & -1.156656 & -0.395968 \\
\hline
\end{tabular}

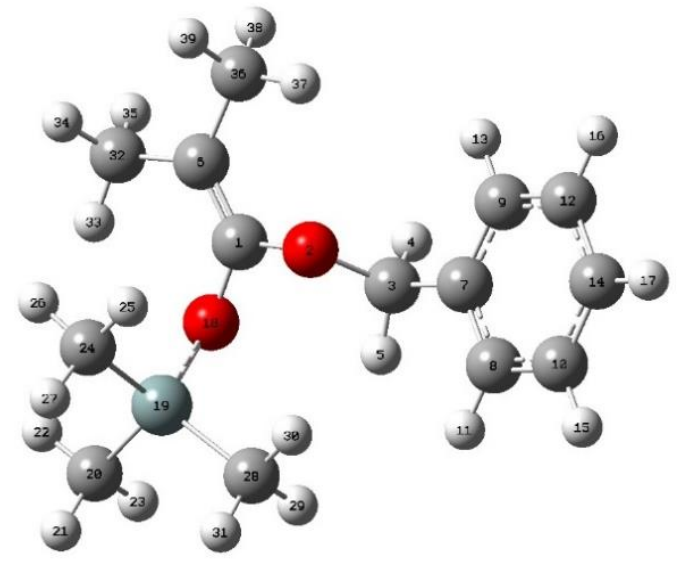

$\underline{2}$

\begin{tabular}{|c|c|c|c|c|c|c|c|c|c|c|c|}
\hline \multicolumn{4}{|c|}{2 (Gas) } & \multicolumn{4}{|c|}{2 (DMSO) } & \multicolumn{4}{|c|}{2 (Toluene) } \\
\hline $\mathrm{C}$ & 1.969582 & 4.736777 & 0.031779 & $\mathrm{C}$ & 1.678954 & 4.484793 & -0.140852 & $\mathrm{C}$ & 1.805864 & 4.416558 & -0.050674 \\
\hline $\mathrm{O}$ & 2.202652 & 5.985744 & -0.477667 & $\mathrm{O}$ & 1.059927 & 5.664544 & -0.451188 & $\mathrm{O}$ & 1.278202 & 5.634523 & -0.392190 \\
\hline $\mathrm{C}$ & 1.669149 & 7.057857 & 0.303938 & $\mathrm{C}$ & 0.354044 & 6.271359 & 0.644763 & $\mathrm{C}$ & 0.555982 & 6.274645 & 0.658505 \\
\hline $\mathrm{H}$ & 2.366384 & 7.887795 & 0.152367 & $\mathrm{H}$ & 1.060848 & 6.422246 & 1.471176 & $\mathrm{H}$ & 1.270649 & 6.583691 & 1.435251 \\
\hline $\mathrm{H}$ & 1.688953 & 6.785433 & 1.365727 & $\mathrm{H}$ & -0.448763 & 5.611248 & 0.987048 & $\mathrm{H}$ & -0.151157 & 5.572500 & 1.113864 \\
\hline $\mathrm{C}$ & 2.976556 & 3.928795 & 0.387635 & $\mathrm{C}$ & 3.006721 & 4.335792 & -0.237885 & $\mathrm{C}$ & 3.125327 & 4.210386 & 0.030439 \\
\hline $\mathrm{C}$ & 0.278620 & 7.482041 & -0.106494 & $\mathrm{C}$ & -0.202871 & 7.584073 & 0.169710 & $\mathrm{C}$ & -0.180946 & 7.469467 & 0.113773 \\
\hline $\mathrm{C}$ & -0.746332 & 7.576729 & 0.833976 & $\mathrm{C}$ & -1.570014 & 7.738771 & -0.069316 & $\mathrm{C}$ & -1.354423 & 7.894495 & 0.741239 \\
\hline $\mathrm{C}$ & 0.014441 & 7.831888 & -1.433276 & $\mathrm{C}$ & 0.654752 & 8.666064 & -0.057315 & $\mathrm{C}$ & 0.297590 & 8.182491 & -0.987416 \\
\hline $\mathrm{C}$ & -2.013814 & 8.021541 & 0.461199 & $\mathrm{C}$ & -2.074913 & 8.955245 & -0.530760 & $\mathrm{C}$ & -2.035125 & 9.020240 & 0.284311 \\
\hline $\mathrm{H}$ & -0.555793 & 7.288455 & 1.864160 & $\mathrm{H}$ & -2.243003 & 6.903407 & 0.106922 & $\mathrm{H}$ & -1.742681 & 7.336313 & 1.589850 \\
\hline $\mathrm{C}$ & -1.253688 & 8.261022 & -1.813324 & $\mathrm{C}$ & 0.155191 & 9.879388 & -0.522861 & $\mathrm{C}$ & -0.387257 & 9.304706 & -1.450026 \\
\hline $\mathrm{H}$ & 0.810046 & 7.760274 & -2.169853 & $\mathrm{H}$ & 1.719064 & 8.553496 & 0.133806 & $\mathrm{H}$ & 1.201491 & 7.850455 & -1.487628 \\
\hline $\mathrm{C}$ & -2.271134 & 8.362588 & -0.864077 & $\mathrm{C}$ & -1.212956 & 10.026016 & -0.760716 & $\mathrm{C}$ & -1.553045 & 9.728999 & -0.814753 \\
\hline $\mathrm{H}$ & -2.802172 & 8.090052 & 1.204348 & $\mathrm{H}$ & -3.140119 & 9.065230 & -0.712094 & $\mathrm{H}$ & -2.947312 & 9.337069 & 0.780975 \\
\hline $\mathrm{H}$ & -1.448242 & 8.524340 & -2.848527 & $\mathrm{H}$ & 0.829908 & 10.712764 & -0.695807 & $\mathrm{H}$ & -0.007733 & 9.849907 & -2.309248 \\
\hline $\mathrm{H}$ & -3.258952 & 8.703503 & -1.158024 & $\mathrm{H}$ & -1.603796 & 10.972508 & -1.122509 & $\mathrm{H}$ & -2.084952 & 10.603732 & -1.176610 \\
\hline $\mathrm{O}$ & 0.653950 & 4.423368 & 0.150848 & $\mathrm{O}$ & 0.837653 & 3.491461 & 0.244342 & $\mathrm{O}$ & 0.876538 & 3.452581 & 0.167873 \\
\hline $\mathrm{Si}$ & -0.286692 & 3.810178 & -1.125449 & $\mathrm{Si}$ & -0.296806 & 2.795082 & -0.827572 & $\mathrm{Si}$ & -0.277980 & 3.014737 & -1.009348 \\
\hline $\mathrm{C}$ & -0.231061 & 1.936173 & -1.046039 & $\mathrm{C}$ & -0.643160 & 1.115678 & -0.089331 & $\mathrm{C}$ & -0.786439 & 1.287099 & -0.511064 \\
\hline $\mathrm{H}$ & -0.876153 & 1.503093 & -1.819154 & $\mathrm{H}$ & -1.392255 & 0.579880 & -0.683823 & $\mathrm{H}$ & -1.557713 & 0.896396 & -1.185012 \\
\hline $\mathrm{H}$ & 0.785427 & 1.564315 & -1.210688 & $\mathrm{H}$ & 0.267038 & 0.506348 & -0.060916 & $\mathrm{H}$ & 0.065508 & 0.599279 & -0.541106 \\
\hline $\mathrm{H}$ & -0.574773 & 1.565996 & -0.074639 & $\mathrm{H}$ & -1.025551 & 1.204629 & 0.933882 & $\mathrm{H}$ & -1.194037 & 1.274138 & 0.505869 \\
\hline $\mathrm{C}$ & 0.410188 & 4.388932 & -2.763849 & $\mathrm{C}$ & 0.509766 & 2.672320 & -2.510626 & $\mathrm{C}$ & 0.555072 & 3.053671 & -2.684104 \\
\hline $\mathrm{H}$ & 0.324300 & 5.473332 & -2.867850 & $\mathrm{H}$ & 0.724197 & 3.661587 & -2.929919 & $\mathrm{H}$ & 0.872155 & 4.069076 & -2.944972 \\
\hline
\end{tabular}




\begin{tabular}{|c|c|c|c|c|c|c|c|c|c|c|c|}
\hline $\mathrm{H}$ & 1.467607 & 4.121375 & -2.859309 & $\mathrm{H}$ & 1.451028 & 2.113586 & -2.454240 & $\mathrm{H}$ & 1.439957 & 2.407596 & -2.701360 \\
\hline $\mathrm{H}$ & -0.134824 & 3.918114 & -3.589823 & $\mathrm{H}$ & -0.150974 & 2.148513 & -3.211611 & $\mathrm{H}$ & -0.130747 & 2.706097 & -3.465677 \\
\hline C & -2.015992 & 4.443581 & -0.824670 & C & -1.842476 & 3.846582 & -0.878479 & $\mathrm{C}$ & -1.739694 & 4.182680 & -0.951225 \\
\hline $\mathrm{H}$ & -2.370365 & 4.159896 & 0.171474 & $\mathrm{H}$ & -2.268796 & 3.971758 & 0.123265 & $\mathrm{H}$ & -2.182607 & 4.226018 & 0.050294 \\
\hline $\mathrm{H}$ & -2.047468 & 5.534748 & -0.899105 & $\mathrm{H}$ & -1.634675 & 4.838148 & -1.294280 & $\mathrm{H}$ & -1.456852 & 5.198370 & -1.246647 \\
\hline $\mathrm{H}$ & -2.710949 & 4.028217 & -1.563013 & $\mathrm{H}$ & -2.604735 & 3.370012 & -1.507177 & $\mathrm{H}$ & -2.521488 & 3.842084 & -1.641201 \\
\hline $\mathrm{C}$ & 2.730346 & 2.538184 & 0.903999 & $\mathrm{C}$ & 3.664385 & 2.999930 & -0.030747 & $\mathrm{C}$ & 3.691855 & 2.841506 & 0.282267 \\
\hline $\mathrm{H}$ & 1.670907 & 2.346867 & 1.079840 & $\mathrm{H}$ & 2.939557 & 2.215825 & 0.198267 & $\mathrm{H}$ & 2.909591 & 2.091475 & 0.414726 \\
\hline $\mathrm{H}$ & 3.106969 & 1.782191 & 0.202313 & $\mathrm{H}$ & 4.223496 & 2.700891 & -0.927073 & $\mathrm{H}$ & 4.333509 & 2.528703 & -0.552022 \\
\hline $\mathrm{H}$ & 3.266817 & 2.386535 & 1.848539 & $\mathrm{H}$ & 4.389876 & 3.046415 & 0.791842 & $\mathrm{H}$ & 4.320755 & 2.840800 & 1.181710 \\
\hline $\mathrm{C}$ & 4.414089 & 4.359748 & 0.290186 & $\mathrm{C}$ & 3.916229 & 5.484780 & -0.573742 & $\mathrm{C}$ & 4.116751 & 5.326925 & -0.143067 \\
\hline $\mathrm{H}$ & 4.510080 & 5.376854 & -0.091805 & $\mathrm{H}$ & 3.369314 & 6.422264 & -0.693839 & $\mathrm{H}$ & 3.628072 & 6.287671 & -0.316695 \\
\hline $\mathrm{H}$ & 4.900433 & 4.306850 & 1.272467 & $\mathrm{H}$ & 4.667749 & 5.622629 & 0.214435 & $\mathrm{H}$ & 4.755294 & 5.419937 & 0.744968 \\
\hline $\mathrm{H}$ & 4.972170 & 3.689852 & -0.376178 & $\mathrm{H}$ & 4.467430 & 5.287971 & -1.502798 & $\mathrm{H}$ & 4.783292 & 5.126218 & -0.991887 \\
\hline
\end{tabular}

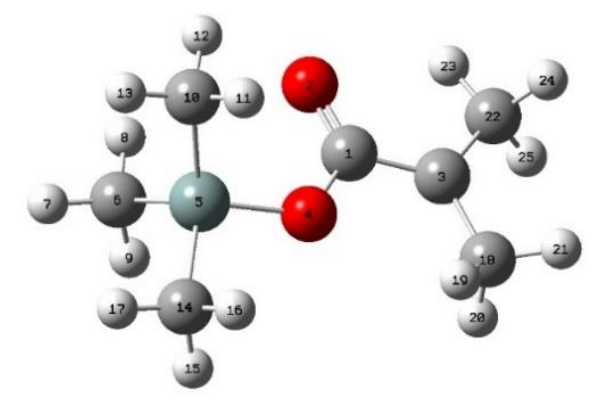

II

\begin{tabular}{|c|c|c|c|c|c|c|c|c|c|c|c|}
\hline \multicolumn{4}{|c|}{ II (Gas) } & \multicolumn{4}{|c|}{ II (DMSO) } & \multicolumn{4}{|c|}{ II (Toluene) } \\
\hline $\mathrm{C}$ & 2.255191 & 3.683955 & -0.545841 & $\mathrm{C}$ & 2.053799 & 4.339274 & -0.317951 & $\mathrm{C}$ & 2.277845 & 3.676624 & -0.563412 \\
\hline $\mathrm{O}$ & 2.641566 & 3.589462 & -1.707425 & $\mathrm{O}$ & 1.866619 & 5.461852 & -0.791308 & $\mathrm{O}$ & 2.667753 & 3.564526 & -1.724166 \\
\hline $\mathrm{C}$ & 3.153806 & 3.772226 & 0.593990 & $\mathrm{C}$ & 3.221001 & 3.973332 & 0.462029 & $\mathrm{C}$ & 3.167850 & 3.779100 & 0.579601 \\
\hline $\mathrm{O}$ & 0.947541 & 3.715106 & -0.229030 & $\mathrm{O}$ & 1.174264 & 3.338393 & -0.485563 & $\mathrm{O}$ & 0.971361 & 3.715772 & -0.249007 \\
\hline $\mathrm{Si}$ & -0.287718 & 3.636368 & -1.426075 & $\mathrm{Si}$ & -0.313199 & 3.495497 & -1.347549 & $\mathrm{Si}$ & -0.285601 & 3.637689 & -1.426066 \\
\hline $\mathrm{C}$ & -0.177209 & 2.008127 & -2.338354 & $\mathrm{C}$ & -1.020480 & 1.772396 & -1.214660 & $\mathrm{C}$ & -0.187375 & 2.014822 & -2.347471 \\
\hline $\mathrm{H}$ & -1.032545 & 1.890224 & -3.013216 & $\mathrm{H}$ & -1.990490 & 1.719594 & -1.723397 & $\mathrm{H}$ & -1.078188 & 1.874854 & -2.971500 \\
\hline $\mathrm{H}$ & 0.741216 & 1.957460 & -2.928874 & $\mathrm{H}$ & -0.357797 & 1.033585 & -1.679103 & $\mathrm{H}$ & 0.692682 & 1.982762 & -2.995957 \\
\hline $\mathrm{H}$ & -0.184971 & 1.165953 & -1.638622 & $\mathrm{H}$ & -1.174297 & 1.482938 & -0.168996 & $\mathrm{H}$ & -0.131790 & 1.169248 & -1.652972 \\
\hline $\mathrm{C}$ & -0.149200 & 5.110332 & -2.568144 & $\mathrm{C}$ & 0.036452 & 3.947889 & -3.126307 & $\mathrm{C}$ & -0.171011 & 5.119268 & -2.558986 \\
\hline $\mathrm{H}$ & -0.141182 & 6.046436 & -2.000162 & $\mathrm{H}$ & 0.393191 & 4.978430 & -3.213390 & $\mathrm{H}$ & -0.160981 & 6.053507 & -1.986277 \\
\hline $\mathrm{H}$ & 0.769220 & 5.057151 & -3.158470 & $\mathrm{H}$ & 0.791971 & 3.282928 & -3.559865 & $\mathrm{H}$ & 0.735454 & 5.083472 & -3.170070 \\
\hline $\mathrm{H}$ & -1.003043 & 5.142755 & -3.254320 & $\mathrm{H}$ & -0.877244 & 3.850302 & -3.725036 & $\mathrm{H}$ & -1.035636 & 5.152874 & -3.232722 \\
\hline $\mathrm{C}$ & -1.841204 & 3.727434 & -0.385724 & $\mathrm{C}$ & -1.403321 & 4.742301 & -0.483672 & $\mathrm{C}$ & -1.817704 & 3.719434 & -0.357755 \\
\hline $\mathrm{H}$ & -1.892848 & 2.890568 & 0.318128 & $\mathrm{H}$ & -1.534791 & 4.483285 & 0.573236 & $\mathrm{H}$ & -1.858853 & 2.878820 & 0.343509 \\
\hline $\mathrm{H}$ & -1.875028 & 4.657759 & 0.190312 & $\mathrm{H}$ & -0.984487 & 5.751405 & -0.540759 & $\mathrm{H}$ & -1.849222 & 4.646421 & 0.225269 \\
\hline $\mathrm{H}$ & -2.733894 & 3.690715 & -1.019895 & $\mathrm{H}$ & -2.396582 & 4.758647 & -0.948594 & $\mathrm{H}$ & -2.722376 & 3.683057 & -0.976039 \\
\hline $\mathrm{C}$ & 2.653055 & 3.890238 & 1.992977 & $\mathrm{C}$ & 3.361632 & 2.603700 & 1.033069 & $\mathrm{C}$ & 2.643642 & 3.944915 & 1.964767 \\
\hline $\mathrm{H}$ & 1.575201 & 4.045478 & 2.030586 & $\mathrm{H}$ & 2.424788 & 2.245904 & 1.471111 & $\mathrm{H}$ & 1.673314 & 4.445266 & 1.981374 \\
\hline $\mathrm{H}$ & 2.891561 & 2.979376 & 2.560607 & $\mathrm{H}$ & 3.638591 & 1.881008 & 0.252017 & $\mathrm{H}$ & 2.510333 & 2.964077 & 2.445850 \\
\hline $\mathrm{H}$ & 3.156035 & 4.716015 & 2.512190 & $\mathrm{H}$ & 4.145847 & 2.581747 & 1.794864 & $\mathrm{H}$ & 3.353325 & 4.506460 & 2.581621 \\
\hline $\mathrm{C}$ & 4.623556 & 3.746895 & 0.353769 & $\mathrm{C}$ & 4.297481 & 4.973243 & 0.690813 & $\mathrm{C}$ & 4.638326 & 3.696927 & 0.366714 \\
\hline $\mathrm{H}$ & 4.853230 & 3.514558 & -0.686668 & $\mathrm{H}$ & 4.150674 & 5.880787 & 0.103121 & $\mathrm{H}$ & 4.886550 & 3.382546 & -0.648111 \\
\hline $\mathrm{H}$ & 5.066501 & 4.722834 & 0.597954 & $\mathrm{H}$ & 4.337184 & 5.249965 & 1.754297 & $\mathrm{H}$ & 5.105778 & 4.675945 & 0.546143 \\
\hline $\mathrm{H}$ & 5.109549 & 3.012845 & 1.008756 & $\mathrm{H}$ & 5.277580 & 4.541869 & 0.450043 & $\mathrm{H}$ & 5.092908 & 3.001885 & 1.084110 \\
\hline
\end{tabular}

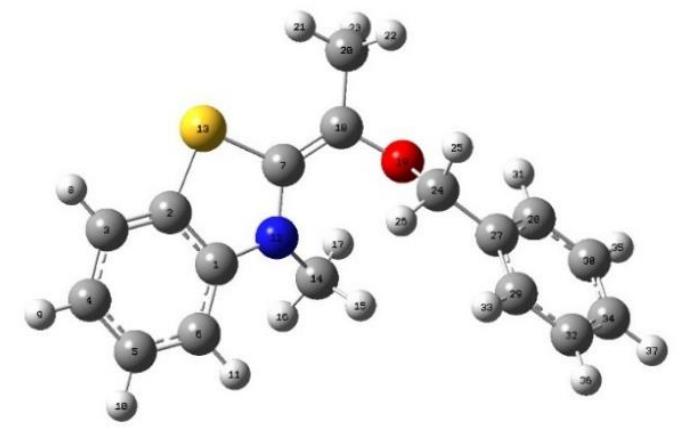

$\underline{3}$

\begin{tabular}{|c|c|c|c|c|c|c|c|c|c|c|c|}
\hline \multicolumn{4}{|c|}{3 (Gas) } & \multicolumn{4}{|c|}{3 (DMSO) } & \multicolumn{4}{|c|}{3 (Toluene) } \\
\hline $\mathrm{C}$ & -1.026415 & -2.312636 & -0.585745 & $\mathrm{C}$ & -1.075353 & -2.013871 & -0.556571 & $\mathrm{C}$ & -1.076089 & -2.063406 & -0.534820 \\
\hline $\mathrm{C}$ & -2.296632 & -2.004640 & -0.075578 & $\mathrm{C}$ & -2.257145 & -1.860058 & 0.187623 & $\mathrm{C}$ & -2.354203 & -1.89078 & 0.016036 \\
\hline $\mathrm{C}$ & -2.694419 & -0.692436 & 0.117794 & $\mathrm{C}$ & -2.533481 & -0.691480 & 0.878585 & $\mathrm{C}$ & -2.822198 & -0.64051 & 0.382427 \\
\hline $\mathrm{C}$ & -1.806383 & 0.337014 & -0.208791 & $\mathrm{C}$ & -1.603912 & 0.353642 & 0.831865 & $\mathrm{C}$ & -1.994455 & 0.470750 & 0.191112 \\
\hline $\mathrm{C}$ & -0.546202 & 0.037407 & -0.717871 & $\mathrm{C}$ & -0.425965 & 0.206496 & 0.101782 & $\mathrm{C}$ & -0.724990 & 0.307634 & -0.356602 \\
\hline $\mathrm{C}$ & -0.144390 & -1.285995 & -0.914060 & $\mathrm{C}$ & -0.146774 & -0.972743 & -0.594121 & C & -0.251488 & -0.954226 & -0.724222 \\
\hline $\mathrm{C}$ & -1.905252 & -4.471794 & -0.418434 & $\mathrm{C}$ & -1.982544 & -4.147906 & -0.847064 & $\mathrm{C}$ & -1.794275 & -4.294676 & -0.575527 \\
\hline $\mathrm{H}$ & -3.677292 & -0.467778 & 0.519001 & $\mathrm{H}$ & -3.454861 & -0.585289 & 1.443271 & $\mathrm{H}$ & -3.813005 & -0.52359 & 0.810145 \\
\hline $\mathrm{H}$ & -2.104333 & 1.369874 & -0.063818 & $\mathrm{H}$ & -1.803314 & 1.274193 & 1.370806 & $\mathrm{H}$ & -2.347359 & 1.457793 & 0.470556 \\
\hline
\end{tabular}




\begin{tabular}{|c|c|c|c|c|c|c|c|c|c|c|c|}
\hline $\mathrm{H}$ & 0.138307 & 0.839643 & -0.974020 & $\mathrm{H}$ & 0.296948 & 1.016374 & 0.076029 & $\mathrm{H}$ & -0.086088 & 1.173015 & -0.502964 \\
\hline $\mathrm{H}$ & 0.833422 & -1.506554 & -1.328380 & $\mathrm{H}$ & 0.783763 & -1.078839 & -1.141436 & $\mathrm{H}$ & 0.739000 & -1.063687 & -1.151585 \\
\hline $\mathrm{N}$ & -0.782051 & -3.676685 & -0.689434 & $\mathrm{~N}$ & -0.976799 & -3.237080 & -1.201644 & $\mathrm{~N}$ & -0.761543 & -3.380780 & -0.827848 \\
\hline $\mathrm{S}$ & -3.204981 & -3.472169 & 0.294300 & $\mathrm{~S}$ & -3.276475 & -3.298332 & 0.056683 & $\mathrm{~S}$ & -3.187835 & -3.437492 & 0.150446 \\
\hline $\mathrm{C}$ & 0.576861 & -4.198885 & -0.687631 & $\mathrm{C}$ & -0.114748 & -3.431990 & -2.357027 & $\mathrm{C}$ & 0.591332 & -3.728999 & -1.227347 \\
\hline $\mathrm{H}$ & 0.956853 & -4.369748 & -1.700933 & $\mathrm{H}$ & -0.655870 & -3.990842 & -3.123042 & $\mathrm{H}$ & 0.806303 & -3.361793 & -2.237959 \\
\hline $\mathrm{H}$ & 1.227455 & -3.483429 & -0.176705 & $\mathrm{H}$ & 0.143728 & -2.455736 & -2.773532 & $\mathrm{H}$ & 1.306930 & -3.282717 & -0.528214 \\
\hline $\mathrm{H}$ & 0.601137 & -5.146638 & -0.152818 & $\mathrm{H}$ & 0.802310 & -3.970339 & -2.106049 & $\mathrm{H}$ & 0.710799 & -4.807355 & -1.213219 \\
\hline $\mathrm{C}$ & -2.049585 & -5.777604 & -0.709617 & $\mathrm{C}$ & -1.992768 & -5.477845 & -1.060376 & $\mathrm{C}$ & -1.865925 & -5.619147 & -0.816045 \\
\hline $\mathrm{O}$ & -1.021842 & -6.442316 & -1.362142 & $\mathrm{O}$ & -0.855518 & -6.065530 & -1.573590 & $\mathrm{O}$ & -0.806934 & -6.280847 & -1.414389 \\
\hline $\mathrm{C}$ & -3.229916 & -6.626364 & -0.360882 & $\mathrm{C}$ & -3.129117 & -6.387160 & -0.704178 & $\mathrm{C}$ & -3.061033 & -6.462248 & -0.472632 \\
\hline $\mathrm{H}$ & -4.073542 & -6.030894 & -0.001349 & $\mathrm{H}$ & -3.779573 & -5.940509 & 0.054419 & $\mathrm{H}$ & -3.545446 & -6.134727 & 0.453545 \\
\hline $\mathrm{H}$ & -3.563479 & -7.188495 & -1.240611 & $\mathrm{H}$ & -3.759327 & -6.634837 & -1.566983 & $\mathrm{H}$ & -3.825152 & -6.459660 & -1.261870 \\
\hline $\mathrm{H}$ & -2.974533 & -7.355724 & 0.416686 & $\mathrm{H}$ & -2.738445 & -7.325414 & -0.295869 & $\mathrm{H}$ & -2.738126 & -7.496483 & -0.319943 \\
\hline $\mathrm{C}$ & -1.029228 & -6.238936 & -2.771666 & $\mathrm{C}$ & -1.045019 & -6.783366 & -2.799686 & $\mathrm{C}$ & -1.033331 & -6.587625 & -2.794142 \\
\hline $\mathrm{H}$ & -1.915857 & -6.725691 & -3.205053 & $\mathrm{H}$ & -1.748858 & -7.609752 & -2.643534 & $\mathrm{H}$ & -1.861340 & -7.305468 & -2.878112 \\
\hline $\mathrm{H}$ & -1.096049 & -5.165439 & -2.993488 & $\mathrm{H}$ & -1.467518 & -6.106990 & -3.554542 & $\mathrm{H}$ & -1.320779 & -5.674547 & -3.331083 \\
\hline C & 0.230400 & -6.809652 & -3.369559 & $\mathrm{C}$ & 0.291123 & -7.310247 & -3.244151 & $\mathrm{C}$ & 0.229412 & -7.165842 & -3.372093 \\
\hline $\mathrm{C}$ & 0.829885 & -7.951493 & -2.833789 & $\mathrm{C}$ & 0.784606 & -8.508330 & -2.719022 & $\mathrm{C}$ & 0.727604 & -8.382620 & -2.895808 \\
\hline $\mathrm{C}$ & 0.799488 & -6.206098 & -4.492399 & $\mathrm{C}$ & 1.069553 & -6.597965 & -4.160564 & $\mathrm{C}$ & 0.925402 & -6.495391 & -4.378923 \\
\hline $\mathrm{C}$ & 1.978008 & -8.481351 & -3.417518 & $\mathrm{C}$ & 2.037276 & -8.985669 & -3.099864 & $\mathrm{C}$ & 1.901302 & -8.917993 & -3.417240 \\
\hline $\mathrm{H}$ & 0.398149 & -8.413598 & -1.952105 & $\mathrm{H}$ & 0.182962 & -9.068862 & -2.007705 & $\mathrm{H}$ & 0.193913 & -8.906915 & -2.107786 \\
\hline $\mathrm{C}$ & 1.943700 & -6.739676 & -5.081602 & $\mathrm{C}$ & 2.321896 & -7.074424 & -4.546970 & $\mathrm{C}$ & 2.097106 & -7.034233 & -4.910575 \\
\hline $\mathrm{H}$ & 0.346544 & -5.309107 & -4.908201 & $\mathrm{H}$ & 0.692438 & -5.665651 & -4.573211 & $\mathrm{H}$ & 0.547707 & -5.546558 & -4.751908 \\
\hline C & 2.536456 & -7.879901 & -4.544305 & $\mathrm{C}$ & 2.807964 & -8.269048 & -4.016059 & $\mathrm{C}$ & 2.587491 & -8.245303 & -4.429362 \\
\hline $\mathrm{H}$ & 2.438616 & -9.367658 & -2.991774 & $\mathrm{H}$ & 2.409953 & -9.918607 & -2.687405 & $\mathrm{H}$ & 2.280966 & -9.861913 & -3.037718 \\
\hline $\mathrm{H}$ & 2.376372 & -6.258884 & -5.953548 & $\mathrm{H}$ & 2.915888 & -6.515006 & -5.263742 & $\mathrm{H}$ & 2.625904 & -6.505737 & -5.698139 \\
\hline $\mathrm{H}$ & 3.430523 & -8.295974 & -4.998148 & $\mathrm{H}$ & 3.782772 & -8.641292 & -4.317319 & $\mathrm{H}$ & 3.500217 & -8.666215 & -4.840384 \\
\hline
\end{tabular}

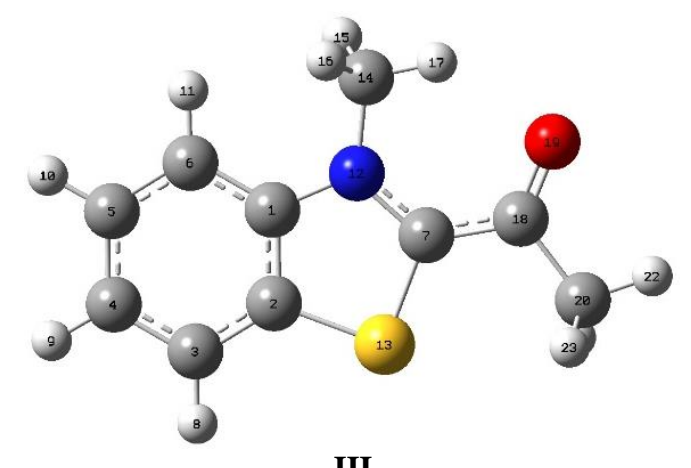

III

\begin{tabular}{|c|c|c|c|c|c|c|c|c|c|c|c|}
\hline \multicolumn{4}{|c|}{ III (Gas) } & \multicolumn{4}{|c|}{ III (DMSO) } & \multicolumn{4}{|c|}{ III (Toluene) } \\
\hline $\mathrm{C}$ & -1.006160 & -2.339018 & -0.460282 & $\mathrm{C}$ & -1.005904 & -2.342380 & -0.471019 & $\mathrm{C}$ & -1.006754 & -2.339407 & -0.468832 \\
\hline $\mathrm{C}$ & -2.342304 & -2.028791 & -0.171720 & $\mathrm{C}$ & -2.339122 & -2.034097 & -0.170139 & $\mathrm{C}$ & -2.340179 & -2.031084 & -0.167725 \\
\hline $\mathrm{C}$ & -2.746656 & -0.718501 & 0.047302 & $\mathrm{C}$ & -2.747302 & -0.724737 & 0.056835 & $\mathrm{C}$ & -2.746538 & -0.721767 & 0.057079 \\
\hline $\mathrm{C}$ & -1.792049 & 0.294692 & -0.024650 & $\mathrm{C}$ & -1.792952 & 0.287989 & -0.021937 & $\mathrm{C}$ & -1.793870 & 0.292232 & -0.023302 \\
\hline $\mathrm{C}$ & -0.459649 & -0.010212 & -0.311149 & $\mathrm{C}$ & -0.460624 & -0.014935 & -0.321793 & $\mathrm{C}$ & -0.463091 & -0.010095 & -0.323511 \\
\hline $\mathrm{C}$ & -0.050742 & -1.322311 & -0.531447 & $\mathrm{C}$ & -0.049978 & -1.325371 & -0.548533 & $\mathrm{C}$ & -0.053028 & -1.320842 & -0.548528 \\
\hline $\mathrm{C}$ & -1.916315 & -4.475451 & -0.524721 & $\mathrm{C}$ & -1.909158 & -4.480049 & -0.533164 & $\mathrm{C}$ & -1.912070 & -4.477763 & -0.526863 \\
\hline $\mathrm{H}$ & -3.783381 & -0.488563 & 0.269376 & $\mathrm{H}$ & -3.782154 & -0.495764 & 0.290078 & $\mathrm{H}$ & -3.781232 & -0.493325 & 0.290442 \\
\hline $\mathrm{H}$ & -2.089410 & 1.324007 & 0.143181 & $\mathrm{H}$ & -2.089807 & 1.317051 & 0.151402 & $\mathrm{H}$ & -2.091233 & 1.321097 & 0.148738 \\
\hline $\mathrm{H}$ & 0.274007 & 0.787519 & -0.364291 & $\mathrm{H}$ & 0.271233 & 0.784703 & -0.379814 & $\mathrm{H}$ & 0.268253 & 0.789597 & -0.383415 \\
\hline $\mathrm{H}$ & 0.985884 & -1.548082 & -0.753545 & $\mathrm{H}$ & 0.985249 & -1.548793 & -0.780873 & $\mathrm{H}$ & 0.982081 & -1.543594 & -0.781365 \\
\hline $\mathrm{N}$ & -0.783987 & -3.692796 & -0.650008 & $\mathrm{~N}$ & -0.782130 & -3.697503 & -0.662225 & $\mathrm{~N}$ & -0.783250 & -3.694170 & -0.657086 \\
\hline $\mathrm{S}$ & -3.320187 & -3.483507 & -0.142330 & $\mathrm{~S}$ & -3.313109 & -3.491539 & -0.132563 & $\mathrm{~S}$ & -3.314706 & -3.488194 & -0.127483 \\
\hline $\mathrm{C}$ & 0.554260 & -4.185285 & -0.950069 & $\mathrm{C}$ & 0.568646 & -4.182851 & -0.920620 & $\mathrm{C}$ & 0.562263 & -4.184530 & -0.925704 \\
\hline $\mathrm{H}$ & 0.910373 & -3.731103 & -1.880337 & $\mathrm{H}$ & 0.945199 & -3.738191 & -1.846366 & $\mathrm{H}$ & 0.933679 & -3.745573 & -1.856834 \\
\hline $\mathrm{H}$ & 1.232994 & -3.917080 & -0.133997 & $\mathrm{H}$ & 1.222301 & -3.897191 & -0.091082 & $\mathrm{H}$ & 1.225152 & -3.902395 & -0.101587 \\
\hline $\mathrm{H}$ & 0.510657 & -5.265937 & -1.058101 & $\mathrm{H}$ & 0.540734 & -5.263792 & -1.017599 & $\mathrm{H}$ & 0.528869 & -5.266054 & -1.020747 \\
\hline $\mathrm{C}$ & -2.014824 & -5.895795 & -0.671064 & $\mathrm{C}$ & -2.025284 & -5.894410 & -0.701610 & $\mathrm{C}$ & -2.020657 & -5.894131 & -0.694678 \\
\hline $\mathrm{O}$ & -1.036947 & -6.618118 & -0.943475 & $\mathrm{O}$ & -1.060189 & -6.627571 & -1.019411 & $\mathrm{O}$ & -1.051831 & -6.618687 & -1.004822 \\
\hline $\mathrm{C}$ & -3.386466 & -6.514456 & -0.469014 & $\mathrm{C}$ & -3.394843 & -6.500638 & -0.471301 & $\mathrm{C}$ & -3.388525 & -6.509467 & -0.471196 \\
\hline $\mathrm{H}$ & -4.141981 & -6.061219 & -1.120758 & $\mathrm{H}$ & -4.157486 & -6.053634 & -1.120065 & $\mathrm{H}$ & -4.153963 & -6.058033 & -1.113235 \\
\hline $\mathrm{H}$ & -3.312414 & -7.577811 & -0.697903 & $\mathrm{H}$ & -3.341820 & -7.568924 & -0.687835 & $\mathrm{H}$ & -3.325733 & -7.574057 & -0.701006 \\
\hline $\mathrm{H}$ & -3.724971 & -6.398290 & 0.567079 & $\mathrm{H}$ & -3.721769 & -6.373480 & 0.567713 & $\mathrm{H}$ & -3.713907 & -6.395866 & 0.569736 \\
\hline
\end{tabular}

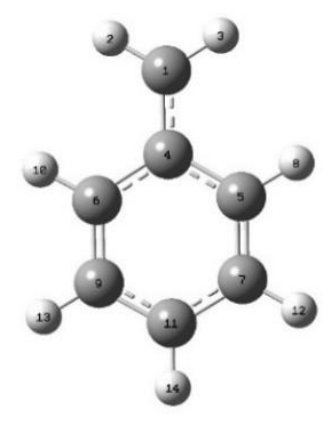


${ }^{-\mathrm{CH}_{2}} \underline{\mathrm{Ph}}$

\begin{tabular}{|c|c|c|c|c|c|c|c|c|c|c|c|}
\hline \multicolumn{4}{|c|}{$\cdot \mathrm{CH}_{2} \mathrm{Ph}$ (Gas) } & \multicolumn{4}{|c|}{$\cdot \mathrm{CH}_{2} \mathrm{Ph}(\mathrm{DMSO})$} & \multicolumn{4}{|c|}{. $\mathrm{CH}_{2} \mathrm{Ph}$ (Toluene) } \\
\hline $\mathrm{C}$ & 1.322645 & 6.764613 & 0.217013 & $\mathrm{C}$ & 1.324170 & 6.764913 & 0.217575 & C & 1.323700 & 6.764909 & 0.217441 \\
\hline $\mathrm{H}$ & 1.980528 & 6.767397 & -0.645292 & $\mathrm{H}$ & 1.982017 & 6.769124 & -0.646377 & $\mathrm{H}$ & 1.981189 & 6.767383 & -0.646222 \\
\hline $\mathrm{H}$ & 1.515408 & 6.044498 & 1.004837 & $\mathrm{H}$ & 1.515894 & 6.044666 & 1.007308 & $\mathrm{H}$ & 1.515577 & 6.043719 & 1.005652 \\
\hline $\mathrm{C}$ & 0.249696 & 7.671203 & 0.311747 & $\mathrm{C}$ & 0.249649 & 7.670873 & 0.311589 & C & 0.250477 & 7.671289 & 0.312005 \\
\hline $\mathrm{C}$ & -0.617984 & 7.674849 & 1.439319 & $\mathrm{C}$ & -0.618047 & 7.673326 & 1.441056 & $\mathrm{C}$ & -0.618244 & 7.674453 & 1.440126 \\
\hline $\mathrm{C}$ & -0.010270 & 8.619370 & -0.716697 & $\mathrm{C}$ & -0.009259 & 8.619516 & -0.718770 & $\mathrm{C}$ & -0.010062 & 8.619700 & -0.717547 \\
\hline $\mathrm{C}$ & -1.672152 & 8.568036 & 1.526745 & C & -1.673164 & 8.567574 & 1.527756 & C & -1.672484 & 8.567828 & 1.526990 \\
\hline $\mathrm{H}$ & -0.441930 & 6.961212 & 2.239471 & $\mathrm{H}$ & -0.441511 & 6.959159 & 2.241197 & $\mathrm{H}$ & -0.442292 & 6.960886 & 2.240754 \\
\hline C & -1.067422 & 9.507920 & -0.618685 & $\mathrm{C}$ & -1.067769 & 9.508490 & -0.620031 & $\mathrm{C}$ & -1.067563 & 9.508007 & -0.619115 \\
\hline $\mathrm{H}$ & 0.637201 & 8.638419 & -1.589013 & $\mathrm{H}$ & 0.638732 & 8.638093 & -1.591231 & $\mathrm{H}$ & 0.637516 & 8.639146 & -1.590134 \\
\hline $\mathrm{C}$ & -1.906534 & 9.490862 & 0.501140 & $\mathrm{C}$ & -1.907465 & 9.491082 & 0.500974 & C & -1.906850 & 9.490928 & 0.501080 \\
\hline $\mathrm{H}$ & -2.320408 & 8.551099 & 2.397585 & $\mathrm{H}$ & -2.322153 & 8.550909 & 2.398719 & $\mathrm{H}$ & -2.321040 & 8.550829 & 2.398080 \\
\hline $\mathrm{H}$ & -1.245227 & 10.222166 & -1.416884 & $\mathrm{H}$ & -1.246215 & 10.223153 & -1.418439 & $\mathrm{H}$ & -1.245508 & 10.222441 & -1.417634 \\
\hline $\mathrm{H}$ & -2.734170 & 10.189042 & 0.573718 & $\mathrm{H}$ & -2.735497 & 10.189811 & 0.573682 & $\mathrm{H}$ & -2.735035 & 10.189168 & 0.573530 \\
\hline
\end{tabular}

\begin{tabular}{|c|c|c|c|c|c|c|c|}
\hline \multicolumn{4}{|c|}{ III (Water) } & \multicolumn{4}{|c|}{ II (Water) } \\
\hline $\mathrm{C}$ & -1.02124100 & 0.54905300 & 0.00106400 & $\mathrm{C}$ & -1.03582600 & -0.38988600 & -0.00553400 \\
\hline $\mathrm{C}$ & -1.14463700 & -0.84546100 & 0.00990000 & $\mathrm{O}$ & -0.82853800 & -1.61245600 & 0.01201100 \\
\hline $\mathrm{C}$ & -2.39052200 & -1.46349700 & -0.00870900 & $\mathrm{C}$ & -2.35404000 & 0.20722600 & -0.00915500 \\
\hline $\mathrm{C}$ & -3.52544600 & -0.65682200 & -0.03854300 & $\mathrm{O}$ & -0.02922200 & 0.49400600 & -0.02069200 \\
\hline $\mathrm{C}$ & -3.40635400 & 0.73765700 & -0.04953300 & $\mathrm{Si}$ & 1.65157800 & 0.05951400 & 0.00036200 \\
\hline $\mathrm{C}$ & -2.16104900 & 1.35703000 & -0.02956700 & $\mathrm{C}$ & 2.47112900 & 1.73417800 & -0.04935400 \\
\hline $\mathrm{C}$ & 1.22497000 & -0.02945000 & 0.01954800 & $\mathrm{H}$ & 3.56140000 & 1.62159100 & -0.03771200 \\
\hline $\mathrm{H}$ & -2.47698700 & -2.54495100 & -0.00135800 & $\mathrm{H}$ & 2.19954100 & 2.28150400 & -0.95841700 \\
\hline $\mathrm{H}$ & -4.50757400 & -1.11681800 & -0.05571400 & $\mathrm{H}$ & 2.18699900 & 2.34302500 & 0.81579100 \\
\hline $\mathrm{H}$ & -4.30063000 & 1.35178400 & -0.07528200 & $\mathrm{C}$ & 2.04029500 & -0.94589400 & -1.52393000 \\
\hline $\mathrm{H}$ & -2.07948300 & 2.43784100 & -0.04400300 & $\mathrm{H}$ & 1.60925800 & -1.94968400 & -1.46646400 \\
\hline $\mathrm{N}$ & 0.29745000 & 0.98600300 & 0.02814300 & $\mathrm{H}$ & 1.65826400 & -0.45498800 & -2.42549200 \\
\hline $\mathrm{S}$ & 0.43478300 & -1.60225200 & 0.04577700 & $\mathrm{H}$ & 3.12628900 & -1.04813300 & -1.63376500 \\
\hline $\mathrm{C}$ & 0.58709600 & 2.41464400 & 0.11830200 & $\mathrm{C}$ & 2.01805800 & -0.84552300 & 1.59082000 \\
\hline $\mathrm{H}$ & 0.29604800 & 2.91183100 & -0.81078200 & $\mathrm{H}$ & 1.69016200 & -0.26179300 & 2.45790800 \\
\hline $\mathrm{H}$ & 0.02168500 & 2.84019900 & 0.95056900 & $\mathrm{H}$ & 1.52329300 & -1.82062900 & 1.62492200 \\
\hline $\mathrm{H}$ & 1.64843000 & 2.55582300 & 0.28821400 & $\mathrm{H}$ & 3.09789500 & -1.00872300 & 1.68685600 \\
\hline $\mathrm{C}$ & 2.64318100 & 0.07199500 & -0.04384400 & $\mathrm{C}$ & -2.50905600 & 1.68927100 & 0.00571900 \\
\hline $\mathrm{O}$ & 3.25038500 & 1.17687100 & -0.13944400 & $\mathrm{H}$ & -1.95685700 & 2.14253200 & 0.83704600 \\
\hline $\mathrm{C}$ & 3.43241800 & -1.21692400 & 0.00331200 & $\mathrm{H}$ & -2.11085300 & 2.13727200 & -0.91400500 \\
\hline $\mathrm{H}$ & 3.15609800 & -1.89259100 & -0.81447600 & $\mathrm{H}$ & -3.56192100 & 1.96632700 & 0.09336400 \\
\hline $\mathrm{H}$ & 4.49384200 & -0.98238100 & -0.09010500 & C & -3.56315400 & -0.65655200 & -0.00984700 \\
\hline \multirow[t]{3}{*}{$\mathrm{H}$} & 3.27632700 & -1.75105300 & 0.94747800 & $\mathrm{H}$ & -3.32704500 & -1.71321600 & -0.14118400 \\
\hline & & & & $\mathrm{H}$ & -4.10883500 & -0.53596300 & 0.93644400 \\
\hline & & & & $\mathrm{H}$ & -4.25204400 & -0.34163800 & -0.80322000 \\
\hline
\end{tabular}

\section{B. Mulliken Spin Densities:}

\begin{tabular}{|c|c|c|}
\hline III (Toluene) & III (DMSO) & III (Water) \\
\hline $1 \mathrm{C} \quad-0.055449$ & $1 \mathrm{C} \quad-0.056419$ & $1 \mathrm{C}-0.053572$ \\
\hline $2 \mathrm{C} \quad 0.017083$ & $2 \mathrm{C} \quad 0.021347$ & $2 \mathrm{C} \quad 0.024620$ \\
\hline $\begin{array}{lll}3 \mathrm{C} & -0.009388\end{array}$ & $3 \mathrm{C} \quad-0.013110$ & $3 \mathrm{C} \quad-0.015947$ \\
\hline $4 \mathrm{C} \quad 0.056089$ & $4 \mathrm{C} \quad 0.058455$ & 4 C 0.058792 \\
\hline 5 C -0.024993 & 5 C $\quad-0.026463$ & $\begin{array}{lll}5 \mathrm{C} & -0.025435\end{array}$ \\
\hline $6 \mathrm{C} \quad 0.071121$ & $6 \mathrm{C} \quad 0.070593$ & 6 C 0.066594 \\
\hline 7 C 0.338039 & $7 \mathrm{C} \quad 0.309581$ & $7 \mathrm{C} \quad 0.255967$ \\
\hline $8 \mathrm{H} \quad 0.000413$ & $8 \mathrm{H} \quad 0.000539$ & $8 \mathrm{H} \quad 0.000620$ \\
\hline $9 \mathrm{H} \quad-0.002628$ & $9 \mathrm{H} \quad-0.002682$ & $9 \mathrm{H} \quad-0.002682$ \\
\hline $10 \mathrm{H} \quad 0.000678$ & $10 \mathrm{H} \quad 0.000732$ & $10 \mathrm{H} \quad 0.000694$ \\
\hline $11 \mathrm{H} \quad-0.002787$ & $11 \mathrm{H} \quad-0.002735$ & $11 \mathrm{H}-0.002621$ \\
\hline $12 \mathrm{~N} \quad 0.155721$ & $12 \mathrm{~N} \quad 0.166565$ & $\begin{array}{ll}12 \mathrm{~N} & 0.180119\end{array}$ \\
\hline $13 \mathrm{~S} \quad 0.128964$ & $13 \mathrm{~S} \quad 0.128451$ & $13 \mathrm{~S} \quad 0.131859$ \\
\hline $14 \mathrm{C} \quad-0.012842$ & $14 \mathrm{C} \quad-0.013189$ & $14 \mathrm{C}-0.013300$ \\
\hline $15 \mathrm{H} \quad 0.009280$ & $\begin{array}{lll}15 \mathrm{H} & 0.009398\end{array}$ & $15 \mathrm{H} \quad 0.010669$ \\
\hline $16 \mathrm{H} \quad 0.010062$ & $16 \mathrm{H} \quad 0.010354$ & $16 \mathrm{H} \quad 0.008990$ \\
\hline $17 \mathrm{H} \quad-0.000206$ & $17 \mathrm{H} \quad-0.000095$ & $\begin{array}{lll}17 \mathrm{H} & 0.000122\end{array}$ \\
\hline $18 \mathrm{C} \quad 0.089808$ & $18 \mathrm{C} \quad 0.114990$ & $18 \mathrm{C} \quad 0.170701$ \\
\hline 19 O $\quad 0.238894$ & $19 \mathrm{O} \quad 0.231760$ & $19 \mathrm{O} \quad 0.211246$ \\
\hline $20 \mathrm{C} \quad-0.015628$ & $20 \mathrm{C} \quad-0.017405$ & $20 \mathrm{C}-0.020772$ \\
\hline $21 \mathrm{H} \quad 0.003509$ & $21 \mathrm{H} \quad 0.004315$ & $\begin{array}{lll}21 \mathrm{H} & 0.006307\end{array}$ \\
\hline $22 \mathrm{H} \quad-0.000416$ & $22 \mathrm{H}-0.000393$ & $22 \mathrm{H} \quad-0.000304$ \\
\hline $23 \mathrm{H} \quad 0.004677$ & $23 \mathrm{H} \quad 0.005411$ & $23 \mathrm{H} \quad 0.007333$ \\
\hline
\end{tabular}

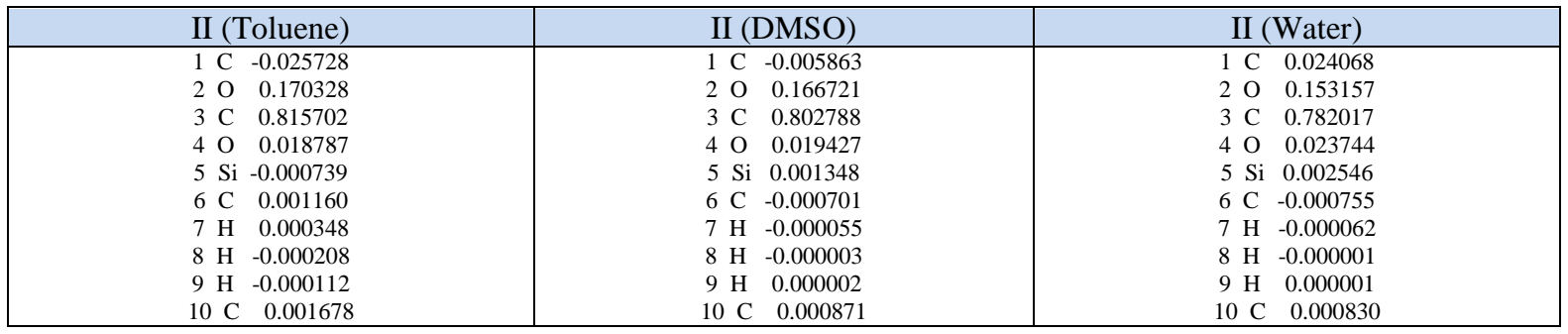




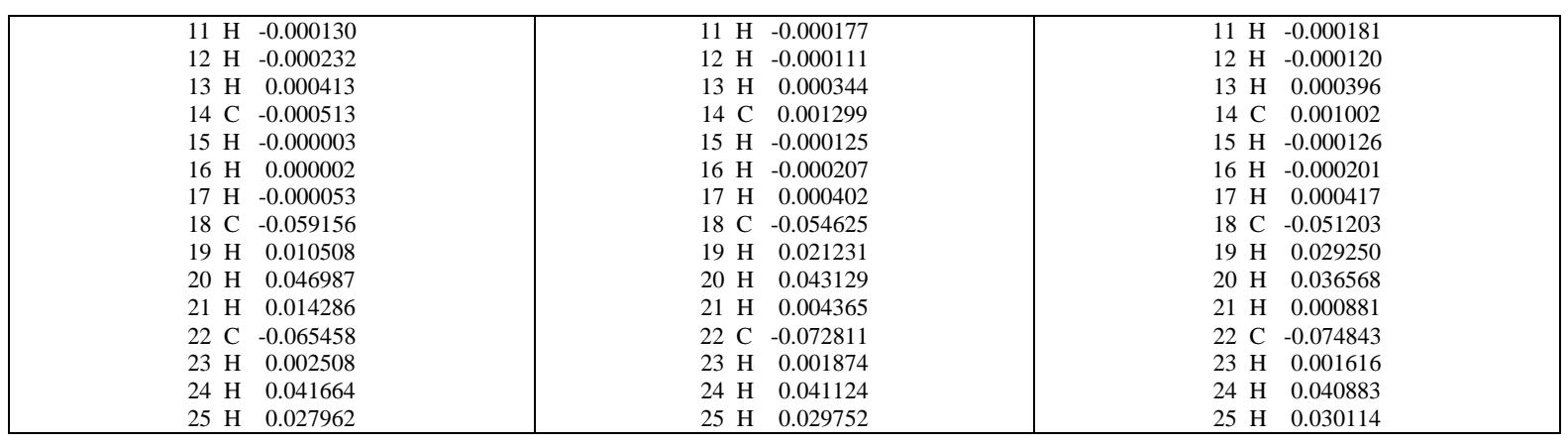

\begin{tabular}{|c|c|c|c|}
\hline$\Delta \mathbf{E}$ & I & II & III \\
\hline Gas & 47.4 & 27.7 & 24.2 \\
\hline Toluene & 45.9 & 25.9 & 21.9 \\
\hline DMSO & 44.9 & 25.7 & 21.5 \\
\hline
\end{tabular}

Table X1: Bond Dissociation Energy for the homolytic fission in different medium. (Unit of $\Delta \mathrm{E}$ is in $\mathrm{kcal} / \mathrm{mol}$ )

\begin{tabular}{|c|c|c|c|}
\hline$\Delta \mathbf{H}$ & I & II & III \\
\hline Gas & 48.0 & 28.5 & 24.2 \\
\hline Toluene & 46.5 & 26.6 & 22.1 \\
\hline DMSO & 45.4 & 26.5 & 21.9 \\
\hline
\end{tabular}

Table X2: Bond Dissociation Enthalpy for the homolytic fission in different medium. (Unit of $\Delta \mathrm{H}$ is in $\mathrm{kcal} / \mathrm{mol}$ )

\begin{tabular}{|c|c|c|c|}
\hline \multicolumn{4}{|c|}{$\begin{array}{l}\text { Table Y1. Mulliken Spin Densities on the } \\
\text { comparable specific atoms for Radical II }\end{array}$} \\
\hline \multirow{2}{*}{$\begin{array}{l}\text { Atom No. } \\
\text { (Element) }\end{array}$} & \multicolumn{3}{|c|}{ Spin Densities } \\
\hline & $\begin{array}{l}\text { Toluene } \\
(\varepsilon=2.374)\end{array}$ & $\begin{array}{c}\text { DMSO } \\
(\varepsilon=46.826)\end{array}$ & $\begin{array}{c}\text { Water } \\
(\varepsilon=78.35553)\end{array}$ \\
\hline $3(\mathrm{C})$ & 0.816 & 0.803 & 0.782 \\
\hline $18(\mathrm{C})$ & -0.059 & -0.055 & -0.051 \\
\hline
\end{tabular}

\begin{tabular}{|c|c|c|c|}
\hline \multicolumn{4}{|c|}{$\begin{array}{l}\text { Table Y2: Mulliken Spin Densities on the } \\
\text { comparable specific atoms for Radical III }\end{array}$} \\
\hline \multirow{2}{*}{$\begin{array}{l}\text { Atom No. } \\
\text { (Element) }\end{array}$} & \multicolumn{3}{|c|}{ Spin Densities } \\
\hline & $\begin{array}{l}\text { Toluene } \\
(\varepsilon=2.374)\end{array}$ & $\begin{array}{c}\text { DMSO } \\
(\varepsilon=46.826)\end{array}$ & $\begin{array}{c}\text { Water } \\
(\varepsilon=78.35553)\end{array}$ \\
\hline $12(\mathrm{~N})$ & 0.156 & 0.167 & 0.180 \\
\hline $7(\mathrm{C})$ & 0.338 & 0.310 & 0.256 \\
\hline
\end{tabular}




\section{Characterization of alcohols:}

3-methyl-1-(1-methyl-1H-benzo[d]imidazol-2-yl)butan-1-ol (S1)

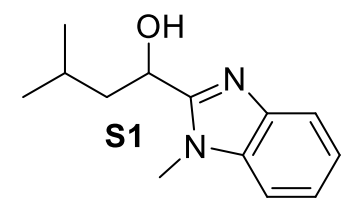

The titled compound was prepared by our previously developed method, ${ }^{2}$ obtained as a solid $\left(760 \mathrm{mg}, 47 \%\right.$ yield). $\mathbf{M P}=128-130{ }^{\circ} \mathrm{C} .{ }^{\mathbf{1}} \mathbf{H} \mathbf{~ N M R}\left(\mathbf{2 0 0} \mathbf{~ M H z}, \mathbf{C D C l}_{\mathbf{3}}\right) \delta 7.67$ $7.58(\mathrm{~m}, 1 \mathrm{H}), 7.22$ - 7.16 (m, 2H), $7.13-7.07$ (m, 1H), 5.28 (br.s, 1H), 4.98 (dd, $J=8.93$, $4.81 \mathrm{~Hz}, 1 \mathrm{H}), 3.69(\mathrm{~s}, 3 \mathrm{H}), 1.98-1.81(\mathrm{~m}, 2 \mathrm{H}), 1.71-1.63(\mathrm{~m}, 1 \mathrm{H}), 0.93(\mathrm{~d}, J=6.87 \mathrm{~Hz}$, $6 \mathrm{H}) .{ }^{13} \mathbf{C}\left(\mathbf{5 0} \mathbf{~ M H z}, \mathbf{C D C l}_{3}\right) \delta 156.3,141.2,135.9,122.5,121.9,119.0,109.1,65.8,44.6$, 30.0, 24.5, 23.2, 21.8. FTIR $\left(\mathbf{c m}^{-1}\right): 3190,3058,3015,2957,2875,1613,1471,1326,1277,1167,1123,1067$, 1010, 917. HRMS(ESI): $\mathrm{m} / \mathrm{z}$ calculated for $\mathrm{C}_{13} \mathrm{H}_{19} \mathrm{ON}_{2}[\mathrm{M}+\mathrm{H}]^{+}:$219.1492, found: 219.1491 .

\section{1-(benzo[d]oxazol-2-yl)-3-methylbutan-1-ol (S2)}

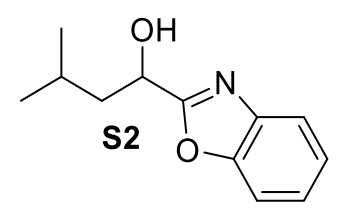

The titled compound was prepared by our previously developed method, ${ }^{2}$ except LiHMDS (1M in THF, 1.25 equiv) as base at $0{ }^{\circ} \mathrm{C}$ for $6 \mathrm{~h}$, obtained as viscous liquid (376 mg, 51\% yield). ${ }^{1} \mathbf{H}$ NMR (400 MHz, $\left.\mathbf{C D C l}_{\mathbf{3}}\right) \delta 7.79-7.62(\mathrm{~m}, 1 \mathrm{H}), 7.58-7.42(\mathrm{~m}, 1 \mathrm{H})$, $7.36-7.29$ (m, 2H), 5.03 (dd, $J=8.70,5.04 \mathrm{~Hz}, 1 \mathrm{H}), 3.85$ (br. s., $1 \mathrm{H}), 1.94-1.84$ (m, $3 \mathrm{H}), 1.00(\mathrm{~d}, J=3.66 \mathrm{~Hz}, 3 \mathrm{H}), 0.98(\mathrm{~d}, J=3.66 \mathrm{~Hz}, 3 \mathrm{H}) .{ }^{13} \mathrm{C}\left(\mathbf{1 0 0 ~ M H z}, \mathbf{C D C l}_{\mathbf{3}}\right) \delta 168.3$, 150.7, 140.4, 125.1, 124.5, 119.9, 110.7, 66.4, 44.5, 24.4, 23.1, 21.8. FTIR (cm-1): 3362, 3012, 2958, 2875, 1661, 1606, 1535, 1460, 1234, 1135, 1074, 1005. HRMS(ESI): m/z calculated for $\mathrm{C}_{12} \mathrm{H}_{16} \mathrm{O}_{2} \mathrm{~N}[\mathrm{M}+\mathrm{H}]+: 206.1176$, found: 206.1175 .

\section{1-(benzo[d]thiazol-2-yl)-3-methylbutan-1-ol (S3)}<smiles>CC(C)CC(O)c1nc2ccccc2s1</smiles>

The titled compound was prepared by our previously developed method, ${ }^{2}$ obtained as a yellow solid (1.91 g, 87\% yield). Spectroscopic data matched that from the previous literature report.

\section{1-(benzo[d]thiazol-2-yl)ethan-1-ol (S4)}

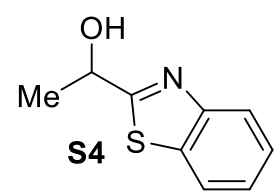

The titled compound was prepared by following procedure $\mathbf{A}$, obtained as a viscous liquid (991mg, 55\% yield). ${ }^{1} \mathbf{H}$ NMR (400 MHz, $\left.\mathbf{C D C l}_{3}\right) \delta 7.89(\mathrm{~d}, J=7.93 \mathrm{~Hz}, 1 \mathrm{H}), 7.79(\mathrm{~d}, J=$ $7.93 \mathrm{~Hz}, 1 \mathrm{H}), 7.38$ (t, $J=7.63 \mathrm{~Hz}, 1 \mathrm{H}), 7.29$ (t, $J=7.94 \mathrm{~Hz}, 1 \mathrm{H}), 5.19$ (q, $J=6.71 \mathrm{~Hz}, 1 \mathrm{H})$, 3.68 (br. s., $1 \mathrm{H}), 1.63(\mathrm{~d}, J=6.10 \mathrm{~Hz}, 3 \mathrm{H}),{ }^{13} \mathbf{C}$ NMR (100 MHz, $\left.\mathbf{C D C l}_{3}\right) \delta$ 177.3, 152.4, 134.6, 126.2, 125.1, 122.7, 121.8, 68.4, 24.0. FTIR $\left(\mathbf{c m}^{-1}\right): 3291,3018,2984,1592,1516$, 1312, 1101, 1071, 1003, 903. HRMS(ESI): m/z calculated for $\mathrm{C}_{9} \mathrm{H}_{10} \mathrm{NOS}[\mathrm{M}+\mathrm{H}]^{+}: 180.0478$, found: 180.0476 .

\section{1-(benzo[d]thiazol-2-yl)-2,2-dimethylpropan-1-ol (S5)}

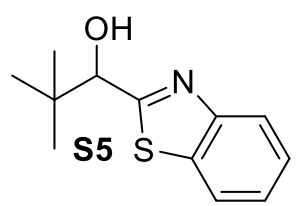

The titled compound was prepared by our previously developed method, ${ }^{2}$ obtained as a white solid (2.61 g, 80\% yield). $\mathbf{M P}=102-103{ }^{\circ} \mathrm{C} .{ }^{1} \mathbf{H}$ NMR $\left(400 \mathbf{~ M H z}, \mathbf{C D C l}_{\mathbf{3}}\right) \delta 7.90(\mathrm{~d}$, $J=7.79 \mathrm{~Hz}, 1 \mathrm{H}), 7.82(\mathrm{~d}, J=8.01 \mathrm{~Hz}, 1 \mathrm{H}), 7.40(\mathrm{t}, J=7.56 \mathrm{~Hz}, 1 \mathrm{H}), 7.34(\mathrm{t}, J=7.56 \mathrm{~Hz}$, $1 \mathrm{H}), 4.65(\mathrm{~d}, J=4.58 \mathrm{~Hz}, 1 \mathrm{H}), 4.51(\mathrm{~d}, J=5.04 \mathrm{~Hz}, 1 \mathrm{H}), 1.00(\mathrm{~s}, 9 \mathrm{H}) .{ }^{13} \mathbf{C}$ NMR (100 MHz, $\left.\mathbf{C D C l}_{3}\right) \delta 174.7,152.1,134.7,125.9,124.9,122.7,121.5,79.7,35.9,25.8$. FTIR $\left(\mathbf{c m}^{-1}\right)$ : 3412, 3016, 2965, 2874, 1637, 1506, 1448, 1309, 1230, 1174, 1069, 1018, 902. HRMS(ESI): m/z calculated for $\mathrm{C}_{12} \mathrm{H}_{16} \mathrm{ONS}[\mathrm{M}+\mathrm{H}]^{+}: 222.0947$, found: 222.0947 . 


\section{1-(benzo[d]thiazol-2-yl)-2-phenylethan-1-ol (S6)}

The titled compound was prepared by our previously developed method, ${ }^{2}$ obtained as<smiles>OC(Cc1ccccc1)c1nc2ccccc2s1</smiles>
a yellow solid (1.96 g, 77\% yield). Spectroscopic data matched that from the previous literature report. ${ }^{1}$

\section{1-(benzo[d]thiazol-2-yl)hexan-1-ol (S7)}

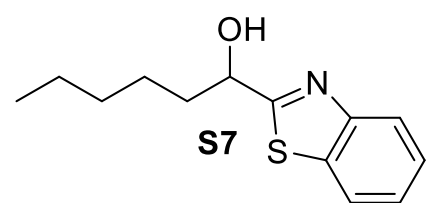

The titled compound was prepared by our previously developed method, ${ }^{2}$ obtained as a white solid $\left(2.70 \mathrm{~g}, 76 \%\right.$ yield). $\mathbf{M P}=58-60{ }^{\circ} \mathrm{C} .{ }^{1} \mathbf{H} \mathbf{~ N M R}\left(\mathbf{2 0 0} \mathbf{~ M H z}, \mathbf{C D C l}_{3}\right) \delta$ $7.79(\mathrm{~d}, J=8.59 \mathrm{~Hz}, 1 \mathrm{H}), 7.67(\mathrm{~d}, J=7.83 \mathrm{~Hz}, 1 \mathrm{H}), 7.35-7.14(\mathrm{~m}, 2 \mathrm{H}), 5.15(\mathrm{~s}$, $1 \mathrm{H}), 4.96(\mathrm{~m}, 1 \mathrm{H}), 1.96-1.65(\mathrm{~m}, 2 \mathrm{H}), 1.51-1.24(\mathrm{~m}, 2 \mathrm{H}), 1.22-1.05(\mathrm{~m}, 4 \mathrm{H})$, $0.82-0.64(\mathrm{~m}, 3 \mathrm{H}) .{ }^{13} \mathbf{C}\left(\mathbf{5 0} \mathbf{~ M H z}, \mathbf{C D C l}_{3}\right) \delta 178.0,152.5,134.4,125.8,124.7$ 122.4, 121.6, 71.8, 38.0, 31.4, 24.9, 22.4, 13.8. FTIR $\left(\mathbf{c m}^{-1}\right): 3393,2933,2863,1641,1513,1450,1314,1168$, 1121, 1067, 927. HRMS(ESI): $\mathrm{m} / \mathrm{z}$ calculated for $\mathrm{C}_{13} \mathrm{H}_{18} \mathrm{ONS}[\mathrm{M}+\mathrm{H}]^{+}: 236.1104$, found: 236.1103 .

\section{Benzo[d]thiazol-2-yl(cyclohexyl)methanol (S8)}<smiles>OC(c1nc2ccccc2s1)C1CCCCC1</smiles>

The titled compound was prepared by our previously developed method, ${ }^{2}$ obtained as a white solid (2.93 g, 79\% yield). $\mathbf{M P}=132-134{ }^{\circ} \mathbf{C} .{ }^{\mathbf{1}} \mathbf{H} \mathbf{~ N M R}\left(\mathbf{4 0 0} \mathbf{~ M H z}, \mathbf{C D C l}_{\mathbf{3}}\right) \delta 7.96(\mathrm{~d}$, $J=7.93 \mathrm{~Hz}, 1 \mathrm{H}), 7.86(\mathrm{~d}, J=7.93 \mathrm{~Hz}, 1 \mathrm{H}), 7.45(\mathrm{t}, J=7.63 \mathrm{~Hz}, 1 \mathrm{H}), 7.36(\mathrm{t}, J=7.32 \mathrm{~Hz}$, 1H), 4.86 (s, $1 \mathrm{H}), 3.75$ (br. s, $1 \mathrm{H}), 1.90-1.64(\mathrm{~m}, 6 \mathrm{H}), 1.30-1.11(\mathrm{~m}, 5 \mathrm{H}) .{ }^{\mathbf{1 3}} \mathbf{C ~}(\mathbf{1 0 0}$ MHz, $\left.\mathbf{C D C l}_{3}\right) \delta 175.9,152.5,134.7,126.0,124.9,122.7,121.7,76.5,44.9,29.3,27.0,26.2,26.1,25.8$. FTIR $\left(\mathbf{c m}^{-1}\right):$ 3416, 2921, 2851, 1641, 1508, 1441, 1317, 1277 1176, 1097, 1001. HRMS(ESI): m/z calculated for $\mathrm{C}_{14} \mathrm{H}_{18} \mathrm{ONS}[\mathrm{M}+\mathrm{H}]^{+}: 248.1104$, found: 248.1103 .

\section{Benzo[d]thiazol-2-yl (phenyl)methanol (S9)}

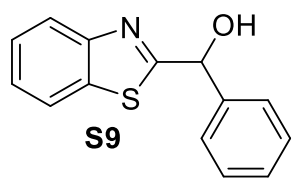

The titled compound was prepared by our previously developed method, ${ }^{2}$ obtained as a yellow solid (1.68 g, 70\% yield). Spectroscopic data matched that from the previous literature report. $^{2}$

\section{Benzo[d]thiazol-2-yl (4-methoxyphenyl)methanol (S10)}

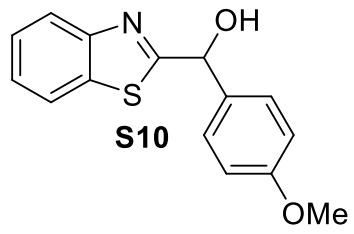

The titled compound was prepared by our previously developed method, ${ }^{2}$ obtained as a white solid (2.03 g, 75\% yield). Spectroscopic data matched that from the previous literature report. $^{2}$

\section{Characterization of ethers:}

\section{2-(1-(benzyloxy)-3-methylbutyl)-1-methyl-1H-benzo[d]imidazole (3a”)}

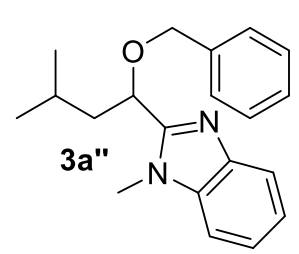

The titled compound was prepared by following the general procedure $\mathbf{C}$ by using $10-15 \%$ EtOAc-pet ether as eluent, obtained as a yellowish liquid (503 mg, 89\% yield). ${ }^{1} \mathbf{H}$ NMR $\left(400 \mathrm{MHz}, \mathbf{C D C l}_{3}\right) \delta 7.79(\mathrm{dd}, J=3.97,2.14 \mathrm{~Hz}, 1 \mathrm{H}), 7.31-7.10(\mathrm{~m}, 8 \mathrm{H}), 5.06-4.91(\mathrm{~m}$, $1 \mathrm{H}), 4.40(\mathrm{dd}, J=11.60,2.44 \mathrm{~Hz}, 1 \mathrm{H}), 4.32(\mathrm{dd}, J=11.60,2.44 \mathrm{~Hz}, 1 \mathrm{H}), 3.73(\mathrm{~d}, J=3.05 \mathrm{~Hz}$, $3 \mathrm{H}), 2.13-2.00(\mathrm{~m}, 1 \mathrm{H}), 1.91-1.78(\mathrm{~m}, 1 \mathrm{H}), 1.74-1.57(\mathrm{~m}, 1 \mathrm{H}), 1.07-0.94(\mathrm{~m}, 3 \mathrm{H}), 0.92$ $0.82(\mathrm{~m}, 3 \mathrm{H}) .{ }^{13} \mathbf{C}$ NMR (100 MHz, $\left.\mathbf{C D C l}_{3}\right) \delta$ 152.9, 141.5, 136.9, 136.0, 127.6, 127.1, 127.0, 122.0, 121.3, 
119.0, 108.5, 74.5, 70.2, 42.3, 29.6, 24.1, 22.4, 21.2. FTIR (cm $\left.{ }^{-1}\right): 3019,2954,2874,1467,1396,1326,1083$, 1026, 912. HRMS(ESI): $\mathrm{m} / \mathrm{z}$ calculated for $\mathrm{C}_{20} \mathrm{H}_{25} \mathrm{ON}_{2}[\mathrm{M}+\mathrm{H}]^{+}: 309.1961$, found: 309.1959.

\section{2-(1-(benzyloxy)-3-methylbutyl)benzo[d]oxazole (3a')}

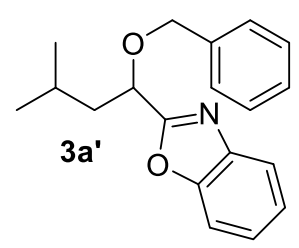

The titled compound was prepared by following the general procedure $\mathbf{C}$, obtained as a yellowish liquid (392 mg, 88\% yield). ${ }^{1} \mathbf{H}$ NMR (500 $\left.\mathbf{~ M H z , ~} \mathbf{C D C l}_{\mathbf{3}}\right) \delta 7.80-7.69(\mathrm{~m}, 1 \mathrm{H})$, $7.60-7.51(\mathrm{~m}, 1 \mathrm{H}), 7.39-7.28(\mathrm{~m}, 6 \mathrm{H}), 7.28-7.23(\mathrm{~m}, 1 \mathrm{H}), 4.76(\mathrm{dd}, J=8.58,5.15 \mathrm{~Hz}, 1 \mathrm{H})$, $4.64(\mathrm{~d}, J=11.83 \mathrm{~Hz}, 1 \mathrm{H}), 4.47(\mathrm{~d}, J=11.45 \mathrm{~Hz}, 1 \mathrm{H}), 2.16-2.01(\mathrm{~m}, 1 \mathrm{H}), 1.87-1.71(\mathrm{~m}$, 2H), $0.95(\mathrm{~d}, J=6.48 \mathrm{~Hz}, 3 \mathrm{H}), 0.85(\mathrm{~d}, J=6.49 \mathrm{~Hz}, 3 \mathrm{H}) .{ }^{13} \mathbf{C} \mathbf{N M R}\left(\mathbf{1 2 5} \mathbf{M H z}, \mathbf{C D C l}_{\mathbf{3}}\right) \delta$ 165.7, 150.7, 140.6, 137.3, 128.3, 128.0, 127.7, 125.1, 124.3, 120.1, 110.8, 73.0, 71.6, 42.7, 24.3, 22.9, 21.8. HRMS(ESI): $\mathrm{m} / \mathrm{z}$ calculated for $\mathrm{C}_{19} \mathrm{H}_{22} \mathrm{O}_{2} \mathrm{~N}[\mathrm{M}+\mathrm{H}]^{+}: 296.1645$, found: 296.1637 .

\section{2-(1-(benzyloxy)-3-methylbutyl)benzo[d]thiazole (3a)}<smiles>CC(C)CC(OCc1ccccc1)c1nc2ccccc2s1</smiles>

The titled compound was prepared by following the general procedure $\mathbf{C}$, obtained as a yellowish liquid (1.25 g, 89\% yield). ${ }^{1} \mathbf{H} \mathbf{N M R}\left(\mathbf{5 0 0} \mathbf{~ M H z}, \mathbf{C D C l}_{3}\right) \delta 8.02(\mathrm{~d}, J=8.01 \mathrm{~Hz}$, $1 \mathrm{H}), 7.91(\mathrm{~d}, J=8.01 \mathrm{~Hz}, 1 \mathrm{H}), 7.52(\mathrm{t}, J=7.25 \mathrm{~Hz}, 1 \mathrm{H}), 7.42-7.28(\mathrm{~m}, 6 \mathrm{H}), 4.90(\mathrm{dd}, J=$ 9.16, $4.58 \mathrm{~Hz}, 1 \mathrm{H}), 4.67(\mathrm{~d}, J=11.44 \mathrm{~Hz}, 1 \mathrm{H}), 4.48(\mathrm{~d}, J=11.44 \mathrm{~Hz}, 1 \mathrm{H}), 1.82-2.01(\mathrm{~m}$, 2H), $1.69(\mathrm{~m}, 1 \mathrm{H}), 0.96(\mathrm{~d}, J=6.87 \mathrm{~Hz}, 3 \mathrm{H}), 0.89(\mathrm{~d}, J=6.48 \mathrm{~Hz}, 3 \mathrm{H}) .{ }^{13} \mathbf{C}$ NMR $(\mathbf{1 0 0}$ MHz, $\left.\mathbf{C D C l}_{3}\right) \delta 176.0,153.0,134.9,128.6,128.4,128.1,127.9,125.9,125.0,122.9$,

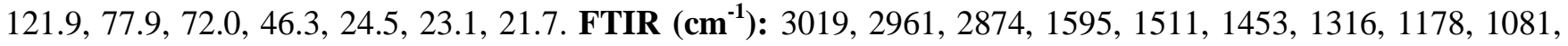
1022, 916. HRMS(ESI): $\mathrm{m} / \mathrm{z}$ calculated for $\mathrm{C}_{19} \mathrm{H}_{21} \mathrm{ONNaS}[\mathrm{M}+\mathrm{Na}]^{+}: 334.1236$, found: 334.1230 .

\section{2-(1-(benzyloxy)ethyl)benzo[d]thiazole (3b)}

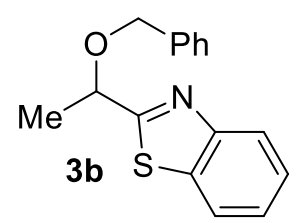

The titled compound was prepared by following the general procedure $\mathbf{C}$, obtained as a yellowish liquid (420 mg, 93\% yield). ${ }^{1} \mathbf{H}$ NMR (500 $\left.\mathbf{~ M H z , ~} \mathbf{C D C l}_{3}\right) \delta 8.00(\mathrm{~d}, J=8.01 \mathrm{~Hz}$, $1 \mathrm{H}), 7.91(\mathrm{~d}, J=7.63 \mathrm{~Hz}, 1 \mathrm{H}), 7.48(\mathrm{t}, J=8.20 \mathrm{~Hz}, 1 \mathrm{H}), 7.42-7.33(\mathrm{~m}, 5 \mathrm{H}), 7.33-7.28(\mathrm{~m}$, 1H), $4.98(\mathrm{q}, J=6.49 \mathrm{~Hz}, 1 \mathrm{H}), 4.68(\mathrm{~d}, J=11.83 \mathrm{~Hz}, 1 \mathrm{H}), 4.58(\mathrm{~d}, J=11.44 \mathrm{~Hz}, 1 \mathrm{H}), 1.68$ $(\mathrm{d}, J=6.87 \mathrm{~Hz}, 3 \mathrm{H}) .{ }^{13} \mathbf{C}$ NMR $\left(\mathbf{C D C l}_{3}, \mathbf{1 2 5} \mathbf{M H z}\right) \delta 176.0,153.1,137.5,135.0,128.5$, 127.9, 127.8, 125.9, 125.1, 123.0, 121.9, 75.6, 71.7, 22.5. FTIR $\left(\mathbf{c m}^{-1}\right): 3019,2983,2932,2871,1514,1447$, 1377, 1312, 1097, 1035, 976, 912. HRMS(ESI): $\mathrm{m} / \mathrm{z}$ calculated for $\mathrm{C}_{16} \mathrm{H}_{16} \mathrm{ONS}[\mathrm{M}+\mathrm{H}]^{+}:$270.0947, found: 270.0946.

\section{2-(1-(benzyloxy)-2,2-dimethylpropyl)benzo[d]thiazole (3c)}<smiles>CC(C)(C)C(OCc1ccccc1)c1nc2ccccc2s1</smiles>

The titled compound was prepared by following the general procedure $\mathbf{C}$, obtained as a yellowish liquid (461 mg, 82\% yield). ${ }^{1} \mathbf{H}$ NMR (400 MHz, $\left.\mathbf{C D C l}_{3}\right) \delta 8.04(\mathrm{~d}, J=7.93 \mathrm{~Hz}$, $1 \mathrm{H}), 7.90(\mathrm{~d}, J=7.93 \mathrm{~Hz}, 1 \mathrm{H}), 7.47(\mathrm{t}, J=7.63 \mathrm{~Hz}, 1 \mathrm{H}), 7.43-7.25(\mathrm{~m}, 6 \mathrm{H}), 4.65(\mathrm{~d}, J=$ $11.60 \mathrm{~Hz}, 1 \mathrm{H}), 4.49(\mathrm{~s}, 1 \mathrm{H}), 4.41(\mathrm{~d}, J=11.60 \mathrm{~Hz}, 1 \mathrm{H}), 1.06(\mathrm{~s}, 9 \mathrm{H}) .{ }^{13} \mathbf{C}$ NMR $(\mathbf{1 0 0} \mathbf{~ M H z}$, $\left.\mathbf{C D C l}_{3}\right) \delta 173.2,152.9,137.7,135.1,128.3,127.9,127.7,125.8,125.0,123.0,121.6,87.4$, 72.6, 35.9, 26.3. HRMS(ESI): $\mathrm{m} / \mathrm{z}$ calculated for $\mathrm{C}_{19} \mathrm{H}_{22} \mathrm{ONS}[\mathrm{M}+\mathrm{H}]^{+}: 312.1417$, found:

312.1415 .

\section{2-(1-(benzyloxy)-2-phenylethyl)benzo[d]thiazole (3d)}<smiles>BrC(OCc1ccccc1)c1nc2ccccc2s1</smiles>

The titled compound was prepared by following the general procedure $\mathbf{C}$, obtained as a yellowish liquid (353 mg, 87\% yield). ${ }^{1} \mathbf{H}$ NMR (400 $\left.\mathbf{~ M H z}, \mathbf{C D C l}_{3}\right) \delta 8.02(\mathrm{~d}, J=8.39 \mathrm{~Hz}$, $1 \mathrm{H}), 7.90(\mathrm{~d}, J=8.39 \mathrm{~Hz}, 1 \mathrm{H}), 7.51-7.45(\mathrm{~m}, 1 \mathrm{H}), 7.42-7.36(\mathrm{~m}, 1 \mathrm{H}), 7.30-7.20(\mathrm{~m}, 8 \mathrm{H})$, 
$7.18-7.12(\mathrm{~m}, 2 \mathrm{H}), 5.05-5.01(\mathrm{~m}, 1 \mathrm{H}), 4.63(\mathrm{~d}, J=11.44 \mathrm{~Hz}, 1 \mathrm{H}), 4.40(\mathrm{~d}, J=11.44 \mathrm{~Hz}, 1 \mathrm{H}), 3.36-3.16(\mathrm{~m}$, 2H). ${ }^{13} \mathbf{C}$ NMR (100 MHz, $\left.\mathbf{C D C l}_{3}\right) \delta 174.7,153.1,137.2,137.1,134.9,129.6,128.8,128.3,128.3,127.8,127.7$, 127.1, 126.6, 126.0, 125.1, 123.0, 121.9, 80.4, 72.3, 43.4. FTIR $\left(\mathbf{c m}^{-1}\right): 3022,2980,2926,2869,1676,1596$, 1507, 1447, 1380, 1320, 1171, 1083, 1023, 930. HRMS(ESI): $\mathrm{m} / \mathrm{z}$ calculated for $\mathrm{C}_{22} \mathrm{H}_{20} \mathrm{ONS}[\mathrm{M}+\mathrm{H}]^{+}: 346.1260$, found: 346.1263 .

\section{2-(1-(benzyloxy)hexyl)benzo[d]thiazole (3e)}

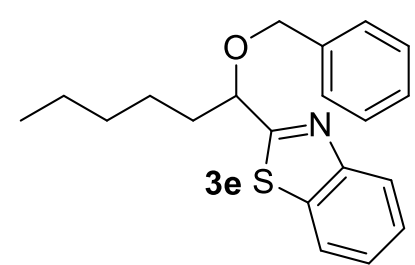

The titled compound was prepared by following the general procedure $\mathbf{C}$, obtained as a yellowish liquid (270 mg, 78\% yield). ${ }^{1} \mathbf{H}$ NMR (500 $\left.\mathbf{~ M H z}, \mathbf{C D C l}_{3}\right) \delta 7.94(\mathrm{~d}, J$ $=8.01 \mathrm{~Hz}, 1 \mathrm{H}), 7.84(\mathrm{~d}, J=7.63 \mathrm{~Hz}, 1 \mathrm{H}), 7.41(\mathrm{t}, J=7.44 \mathrm{~Hz}, 1 \mathrm{H}), 7.36-7.23(\mathrm{~m}$, $6 \mathrm{H}), 4.74(\mathrm{t}, J=6.10 \mathrm{~Hz}, 1 \mathrm{H}), 4.61(\mathrm{~d}, J=11.44 \mathrm{~Hz}, 1 \mathrm{H}), 4.43(\mathrm{~d}, J=11.44 \mathrm{~Hz}$, $1 \mathrm{H}), 1.99-1.88(\mathrm{~m}, 1 \mathrm{H}), 1.88-1.79(\mathrm{~m}, 1 \mathrm{H}), 1.51-1.40(\mathrm{~m}, 1 \mathrm{H}), 1.38-1.27(\mathrm{~m}$, $1 \mathrm{H}), 1.26-1.11(\mathrm{~m}, 4 \mathrm{H}), 0.84-0.72(\mathrm{~m}, 3 \mathrm{H}) .{ }^{13} \mathbf{C}$ NMR $\left(\mathbf{1 2 5} \mathbf{~ M H z}, \mathbf{C D C l}_{3}\right) \delta$ 175.7, 153.1, 137.6, 135.0, 128.4, 128.0, 127.9, 125.9, 125.0, 123.0, 121.9, 79.7, 72.0, 37.1, 31.5, 25.0, 22.5, 14.0. FTIR $\left(\mathbf{c m}^{-1}\right): 3020,2934,2864,1640,1513,1451,1382,1319,1174,1081,1025,922 . \mathbf{H R M S}(\mathrm{ESI}): \mathrm{m} / \mathrm{z}$ calculated for $\mathrm{C}_{20} \mathrm{H}_{24} \mathrm{ONS}[\mathrm{M}+\mathrm{H}]^{+}: 326.1573$. found: 326.1574 .

\section{2-((benzyloxy)(cyclohexyl)methyl)benzo[d]thiazole (3f)}<smiles>c1ccc(COC(c2nc3ccccc3s2)C2CCCCC2)cc1</smiles>

The titled compound was prepared by following the general procedure $\mathbf{C}$, obtained as a yellowish liquid (463 mg, 85\% yield). ${ }^{1} \mathbf{H}$ NMR (500 $\left.\mathbf{~ M H z}, \mathbf{C D C l}_{3}\right) \delta 8.03(\mathrm{~d}, J=8.01 \mathrm{~Hz}$, 1H). $7.90(\mathrm{~d}, J=8.01 \mathrm{~Hz}, 1 \mathrm{H}), 7.47(\mathrm{t}, J=7.63 \mathrm{~Hz}, 1 \mathrm{H}), 7.42-7.26(\mathrm{~m}, 6 \mathrm{H}), 4.65(\mathrm{~d}, J=$ $11.44 \mathrm{~Hz}, 1 \mathrm{H}), 4.55(\mathrm{~d}, J=6.87 \mathrm{~Hz}, 1 \mathrm{H}), 4.44(\mathrm{~d}, J=11.44 \mathrm{~Hz}, 1 \mathrm{H}), 2.06(\mathrm{~d}, J=11.44 \mathrm{~Hz}$, $1 \mathrm{H}), 1.94-1.84(\mathrm{~m}, 1 \mathrm{H}), 1.73(\mathrm{~d}, J=11.44 \mathrm{~Hz}, 2 \mathrm{H}), 1.70-1.58(\mathrm{~m}, 2 \mathrm{H}), 1.48(\mathrm{~d}, J=8.77$ $\mathrm{Hz}, 1 \mathrm{H}), 1.24$ - 1.12 (m, 5H). ${ }^{13} \mathbf{C}$ NMR (125 MHz, $\left.\mathbf{C D C l}_{3}\right) \delta$ 174.7, 153.0, 137.6, 135.0, 128.3, 128.0, 127.8, 125.8, 125.0, 123.0, 121.8, 84.2, 72.3, 44.4, 29.1, 28.8, 26.3, 25.9, 25.8. FTIR (cm $\left.{ }^{-1}\right): 2926$, 2856, 1594, 1510, 1448, 1314, 1158, 1066, 889. HRMS(ESI): $\mathrm{m} / \mathrm{z}$ calculated for $\mathrm{C}_{21} \mathrm{H}_{24} \mathrm{ONS}[\mathrm{M}+\mathrm{H}]^{+}: 338.1573$, found: 338.1572 .

\section{2-((benzyloxy)(phenyl)methyl)benzo[d]thiazole (3g)}<smiles>c1ccc(COC(c2ccccc2)c2nc3ccccc3s2)cc1</smiles>

In an oven dried $50 \mathrm{ml}$ pear-shaped flask with a magnetic bar, alcohol $(500 \mathrm{mg}, 2.07$ mmol, 1.0 equiv.), p-Toluenesulfonic acid (118 mg, $0.621 \mathrm{mmol}), \mathrm{BnOH}(670 \mathrm{mg}, 6.21$ mmol) was taken in dry Toluene $(8 \mathrm{ml})$ at room temperature and degassed for $15 \mathrm{~min}$ and sealed with electrical tape. The reaction container heated to $100{ }^{\circ} \mathrm{C}$ in a oil bath for $48 \mathrm{~h}$ and quenched with saturated $\mathrm{NaHCO}_{3}$ solution.The organic layer was extracted with EtOAc (3 times) and combined organic layer dried over anhydrous $\mathrm{Na}_{2} \mathrm{SO}_{4}$. The solvent removal under reduced pressure gave the crude product which was further purified by column chromatography on silica gel using ethylacetate-pet ether ( $2-4 \%$ EtOAc-pet ether) as eluent to obtain the desired product with (418 mg, 61\% yield). ${ }^{1} \mathbf{H}$ NMR (400 MHz, $\left.\mathbf{C D C l}_{3}\right) \delta 7.99(\mathrm{~d}, J=8.24 \mathrm{~Hz}, 1 \mathrm{H}), 7.85(\mathrm{~d}, J=8.24 \mathrm{~Hz}, 1 \mathrm{H}), 7.59$ $7.52(\mathrm{~m}, 2 \mathrm{H}), 7.46-7.25(\mathrm{~m}, 10 \mathrm{H}), 5.87(\mathrm{~s}, 1 \mathrm{H}), 4.78-4.63(\mathrm{~m}, 2 \mathrm{H}) .{ }^{13} \mathbf{C}$ NMR (100 $\left.\mathbf{~ M H z}, \mathbf{C D C l}_{\mathbf{3}}\right) \delta 173.8$, 153.0, 139.0, 137.2, 135.1, 128.7, 128.4, 127.9, 127.8, 127.1, 125.9, 125.0, 123.2, 121.7, 80.7, 71.2. FTIR (cm $\left.{ }^{1}\right): 3034,2925,2867,1736,1684,1596,1507,1446,1384,1302,1248,1161,1067,1016,912$. HRMS(ESI): m/z calculated for $\mathrm{C}_{21} \mathrm{H}_{18} \mathrm{ONS}[\mathrm{M}+\mathrm{H}]^{+}: 332.1104$, found: 332.1100 .

\section{2-((benzyloxy)(4-methoxyphenyl)methyl)benzo[d]thiazole (3h)}

The titled compound were synthesized by following the literature procedures, for $48 \mathrm{~h}$ at $-25{ }^{\circ} \mathrm{C}$, obtained as a yellowish liquid (1.351g, 97\% yield) by using $4 \%$ acetone-pet ether as a eluent. ${ }^{1} \mathbf{H} \mathbf{~ N M R}\left(\mathbf{4 0 0} \mathbf{~} \mathbf{M H z}, \mathbf{C D C l}_{3}\right) \delta$ 
<smiles>COc1ccc(C(OCc2ccccc2)c2nc3ccccc3s2)cc1</smiles>

$7.98(\mathrm{~d}, J=7.93 \mathrm{~Hz}, 1 \mathrm{H}), 7.87(\mathrm{~d}, J=7.93 \mathrm{~Hz}, 1 \mathrm{H}), 7.52-7.27(\mathrm{~m}, 9 \mathrm{H}), 6.90(\mathrm{~d}, J$ $=8.54 \mathrm{~Hz}, 2 \mathrm{H}), 5.81(\mathrm{~s}, 1 \mathrm{H}), 4.78-4.60(\mathrm{~m}, 2 \mathrm{H}), 3.78(\mathrm{~s}, 3 \mathrm{H}) .{ }^{13} \mathbf{C}$ NMR $(\mathbf{1 0 0}$ $\left.\mathbf{M H z}, \mathbf{C D C l}_{3}\right) \delta 174.2,159.7,153.1,137.4,135.1,131.1,128.5,128.5,127.9$, 127.8, 125.9, 125.0, 123.2, 121.7, 114.1, 80.3, 71.0, 55.3. FTIR $\left(\mathbf{c m}^{-1}\right): 3017,2978$, 1610, 1510, 1452, 1377, 1312, 1173, 1076, 1030, 922. HRMS(ESI): m/z calculated for $\mathrm{C}_{22} \mathrm{H}_{20} \mathrm{O}_{2} \mathrm{NS}[\mathrm{M}+\mathrm{H}]^{+}:$362.1209, found: 362.1204 .

\section{2-(1-((4-chlorobenzyl)oxy)ethyl)benzo[d]thiazole (3i)}

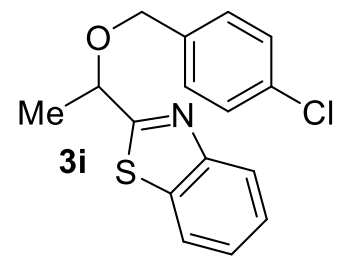

The titled compound was prepared by following the general procedure $\mathbf{C}$, obtained as a yellowish liquid (335 mg, 66\% yield). ${ }^{1} \mathbf{H}$ NMR (400 $\left.\mathbf{~ M H z}, \mathbf{C D C l}_{3}\right) \delta 7.94(\mathrm{~d}, J=7.93$ $\mathrm{Hz}, 1 \mathrm{H}), 7.84(\mathrm{~d}, J=7.93 \mathrm{~Hz}, 1 \mathrm{H}), 7.42(\mathrm{t}, J=7.32 \mathrm{~Hz}, 1 \mathrm{H}), 7.33(\mathrm{t}, J=7.32 \mathrm{~Hz}, 1 \mathrm{H})$, $7.30-7.20(\mathrm{~m}, 4 \mathrm{H}), 4.89(\mathrm{q}, J=6.51 \mathrm{~Hz}, 1 \mathrm{H}), 4.56(\mathrm{~d}, J=12.21 \mathrm{~Hz}, 1 \mathrm{H}), 4.48(\mathrm{~d}, J=$ $11.60 \mathrm{~Hz}, 1 \mathrm{H}), 1.61(\mathrm{~d}, J=6.71 \mathrm{~Hz}, 3 \mathrm{H}) .{ }^{13} \mathbf{C} \mathbf{~ N M R}\left(\mathbf{1 2 5} \mathbf{~ M H z}, \mathbf{C D C l}_{3}\right) \delta 175.5,153.1$, 136.1, 134.9, 133.7, 129.1, 128.6, 126.0, 125.2, 123.0, 121.9, 75.8, 70.8, 22.5. FTIR $\left(\mathbf{c m}^{-}\right.$ 1): 3018, 2983, 2930, 2868, 1596, 1503, 1446, 1377, 1312, 1095, 1023, 976, 933. HRMS(ESI): m/z calculated for $\mathrm{C}_{16} \mathrm{H}_{15} \mathrm{ONClS}[\mathrm{M}+\mathrm{H}]^{+}: 304.0557$, found: 304.0557 .

\section{2-(1-((4-fluorobenzyl)oxy)ethyl)benzo[d]thiazole (3j)}

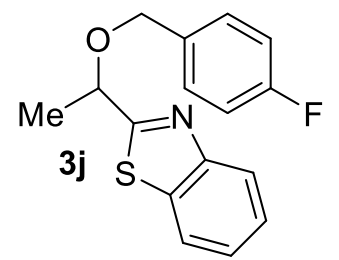

The titled compound was prepared by following the general procedure $\mathbf{C}$, obtained as a yellowish liquid (351 mg, 73\% yield). ${ }^{1} \mathbf{H}$ NMR (400 $\left.\mathbf{~ M H z , ~} \mathbf{C D C l}_{3}\right) \delta 7.94(\mathrm{~d}, J=7.93 \mathrm{~Hz}$, $1 \mathrm{H}), 7.85(\mathrm{~d}, J=7.93 \mathrm{~Hz}, 1 \mathrm{H}), 7.42(\mathrm{t}, J=7.63 \mathrm{~Hz}, 1 \mathrm{H}), 7.38-7.25(\mathrm{~m}, 3 \mathrm{H}), 6.97(\mathrm{t}, J=$ $8.85 \mathrm{~Hz}, 2 \mathrm{H}), 4.89(\mathrm{q}, J=6.31 \mathrm{~Hz}, 1 \mathrm{H}), 4.56(\mathrm{~d}, J=10.99 \mathrm{~Hz}, 1 \mathrm{H}), 4.47(\mathrm{~d}, J=11.60 \mathrm{~Hz}$, $1 \mathrm{H}), 1.61(\mathrm{~d}, J=6.10 \mathrm{~Hz}, 3 \mathrm{H}) .{ }^{13} \mathbf{C}$ NMR $\left(\mathbf{1 2 5} \mathbf{~ M H z}, \mathbf{C D C l}_{\mathbf{3}}\right) \delta 175.7,163.5(\mathrm{C}-\mathrm{F}, 1 \mathrm{JC}-\mathrm{F}=$ $246.1 \mathrm{~Hz}), 161.5(\mathrm{C}-\mathrm{F}, 1 \mathrm{JC}-\mathrm{F}, J=246.1 \mathrm{~Hz}), 153.1,134.9,133.3(\mathrm{C}-\mathrm{F}, 4 \mathrm{JC}-\mathrm{F}=2.9 \mathrm{~Hz})$, $133.4(\mathrm{C}-\mathrm{F}, 4 \mathrm{JC}-\mathrm{F}=2.9 \mathrm{~Hz}), 125.1,126.0,129.7(\mathrm{C}-\mathrm{F}, 3 \mathrm{JC}-\mathrm{F}=7.6 \mathrm{~Hz}), 129.6(\mathrm{C}-\mathrm{F}, 3 \mathrm{JC}-\mathrm{F}=7.6 \mathrm{~Hz}), 126.0$, 125.1, 123.0, 121.9, $115.4(\mathrm{C}-\mathrm{F}, 2 \mathrm{JC}-\mathrm{F}=21.9 \mathrm{~Hz}), 115.2(\mathrm{C}-\mathrm{F}, 2 \mathrm{JC}-\mathrm{F}=21.9 \mathrm{~Hz}), 75.7,70.9,22.5$ (peaks 30 and 206 are from residual acetone). FTIR $\left(\mathbf{c m}^{-1}\right):$ 2970, 2924, 2860, 1604, 1513, 1448, 1375, 1311, 1225, 1160, 1097, 1050, 976. HRMS(ESI): $\mathrm{m} / \mathrm{z}$ calculated for $\mathrm{C}_{16} \mathrm{H}_{15}$ ONFS [M+H] ${ }^{+}: 288.0853$, found: 288.0852 .

\section{2-(1-((4-chlorobenzyl)oxy)-3-methylbutyl)benzo[d]thiazole (3k)}

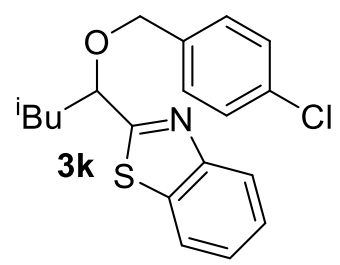

The titled compound was prepared by following the general procedure $\mathbf{C}$, obtained as a yellowish liquid (686 mg, 88\% yield). ${ }^{1} \mathbf{H}$ NMR (400 $\left.\mathbf{M H z}, \mathbf{C D C l}_{3}\right) \delta 8.02(\mathrm{~d}, J=7.93$ $\mathrm{Hz}, 1 \mathrm{H}), 7.90(\mathrm{~d}, J=7.93 \mathrm{~Hz}, 1 \mathrm{H}), 7.48(\mathrm{t}, J=7.32 \mathrm{~Hz}, 1 \mathrm{H}), 7.39(\mathrm{t}, J=7.93 \mathrm{~Hz}, 1 \mathrm{H})$, $7.37-7.24(\mathrm{~m}, 4 \mathrm{H}), 4.88(\mathrm{dd}, J=8.54,4.88 \mathrm{~Hz}, 1 \mathrm{H}), 4.61(\mathrm{~d}, J=11.60 \mathrm{~Hz}, 1 \mathrm{H}), 4.44(\mathrm{~d}, J$ $=11.60 \mathrm{~Hz}, 1 \mathrm{H}), 2.02-1.82(\mathrm{~m}, 2 \mathrm{H}), 1.79-1.64(\mathrm{~m}, 1 \mathrm{H}), 0.97(\mathrm{~d}, J=6.10 \mathrm{~Hz}, 3 \mathrm{H}), 0.91$ $(\mathrm{d}, J=6.71 \mathrm{~Hz}, 3 \mathrm{H}) .{ }^{13} \mathbf{C}$ NMR (100 MHz, $\left.\mathbf{C D C l}_{\mathbf{3}}\right) \delta 175.5,153.0,136.0,134.9,133.7$, 129.3, 128.6, 126.0, 125.1, 123.0, 121.9, 78.2, 71.1, 46.3, 24.6, 23.1, 21.9. FTIR ( $\left.\mathbf{c m}^{-1}\right): 3018,2960,2933,2871$, $1599,1515,1493,1468,1314,1183,1088,1016,928$. HRMS(ESI): $\mathrm{m} / \mathrm{z}$ calculated for $\mathrm{C}_{19} \mathrm{H}_{21} \mathrm{ONClS}[\mathrm{M}+\mathrm{H}]^{+}$: 346.1027, found: 346.1027 .

\section{2-(1-((3-chlorobenzyl)oxy)-3-methylbutyl) benzo[d]thiazole (3l)}

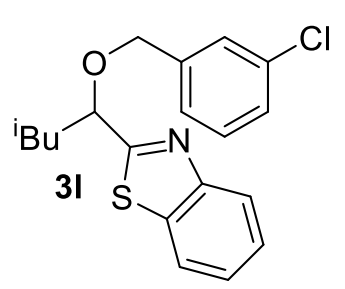

The titled compound was prepared by following the general procedure $\mathbf{C}$, obtained as a yellowish liquid (647 mg, 83\% yield). ${ }^{1} \mathbf{H}$ NMR (400 $\left.\mathbf{M H z}, \mathbf{C D C l}_{3}\right) \delta 8.02$ (d, $J=8.13$ $\mathrm{Hz}, 1 \mathrm{H}), 7.90(\mathrm{~d}, J=7.88 \mathrm{~Hz}, 1 \mathrm{H}), 7.54(\mathrm{~d}, J=9.26 \mathrm{~Hz}, 1 \mathrm{H}), 7.48(\mathrm{t}, J=8.25 \mathrm{~Hz}, 1 \mathrm{H})$, $7.42-7.33(\mathrm{~m}, 2 \mathrm{H}), 7.31-7.21(\mathrm{~m}, 2 \mathrm{H}), 4.95(\mathrm{dd}, J=9.07,4.57 \mathrm{~Hz}, 1 \mathrm{H}), 4.73(\mathrm{~d}, J=$ $12.38 \mathrm{~Hz}, 1 \mathrm{H}), 4.64(\mathrm{~d}, J=12.38 \mathrm{~Hz}, 1 \mathrm{H}), 2.04-1.88(\mathrm{~m}, 2 \mathrm{H}), 1.77-1.68(\mathrm{~m}, 1 \mathrm{H}), 0.97$ 
$(\mathrm{d}, J=6.50 \mathrm{~Hz}, 3 \mathrm{H}), 0.92(\mathrm{~d}, J=6.50 \mathrm{~Hz}, 3 \mathrm{H}) .{ }^{\mathbf{1 3}} \mathbf{C} \mathbf{N M R}\left(\mathbf{1 0 0} \mathbf{~ M H z}, \mathbf{C D C l}_{\mathbf{3}}\right) \delta 175.6,153.1,135.3,135.0,133.2$, 129.6, 129.3, 129.0, 126.8, 126.0, 125.1, 123.0, 121.9, 78.6, 69.2, 46.4, 24.5, 23.2, 21.8. FTIR (cm $\left.{ }^{-1}\right):$ 2958, 2926, 2870, 1587, 1515, 1449, 1316, 1256, 1176, 1090, 1043, 940. HRMS(ESI): m/z calculated for $\mathrm{C}_{19} \mathrm{H}_{21} \mathrm{ONClS}[\mathrm{M}+\mathrm{H}]^{+}:$346.1027, found: 346.1027.

\section{2-(1-((2-chlorobenzyl)oxy)-3-methylbutyl) benzo[d]thiazole (3m)}

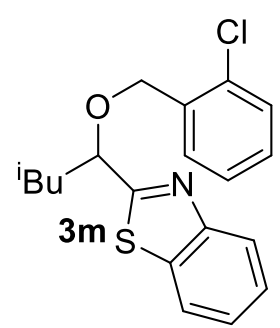

The titled compound was prepared by following the general procedure $\mathbf{C}$, obtained as a yellowish liquid (655mg, 84\% yield). ${ }^{1} \mathbf{H}$ NMR (400 $\left.\mathbf{M H z}, \mathbf{C D C l}_{3}\right) \delta 8.01(\mathrm{~d}, J=8.24 \mathrm{~Hz}$, $1 \mathrm{H}), 7.91(\mathrm{~d}, J=8.70 \mathrm{~Hz}, 1 \mathrm{H}), 7.51-7.46(\mathrm{~m}, 1 \mathrm{H}), 7.42-7.34(\mathrm{~m}, 2 \mathrm{H}), 7.28-7.24(\mathrm{~m}, 2 \mathrm{H})$, $7.24-7.20(\mathrm{~m}, 1 \mathrm{H}), 4.88(\mathrm{dd}, J=9.16,4.58 \mathrm{~Hz}, 1 \mathrm{H}), 4.62(\mathrm{~d}, J=11.45 \mathrm{~Hz}, 1 \mathrm{H}), 4.45(\mathrm{~d}, J=$ $11.91 \mathrm{~Hz}, 1 \mathrm{H}), 2.02-1.84(\mathrm{~m}, 2 \mathrm{H}), 1.74-1.66(\mathrm{~m}, 1 \mathrm{H}), 0.97(\mathrm{~d}, J=6.41 \mathrm{~Hz}, 3 \mathrm{H}), 0.91(\mathrm{~d}, J=$ $6.41 \mathrm{~Hz}, 3 \mathrm{H}) .{ }^{13} \mathbf{C}$ NMR (100 MHz, $\left.\mathbf{C D C l}_{3}\right) \delta 175.4,152.9,139.5,134.9,134.3,129.7,128.0$, 127.9, 126.0, 125.9, 125.2, 123.0, 121.9, 78.3, 71.1, 46.3, 24.5, 23.1, 21.8. FTIR $\left(\mathbf{c m}^{-1}\right): 3019$, 2956, 2873, 1586, 1516, 1467, 1440, 1378, 1317, 1085, 1024, 947, 875. HRMS(ESI): m/z calculated for $\mathrm{C}_{19} \mathrm{H}_{21} \mathrm{ONClS}[\mathrm{M}+\mathrm{H}]^{+}:$346.1027, found: 346.1028 .

\section{2-(1-((4-bromobenzyl)oxy)-3-methylbutyl) benzo[d]thiazole (3n)}

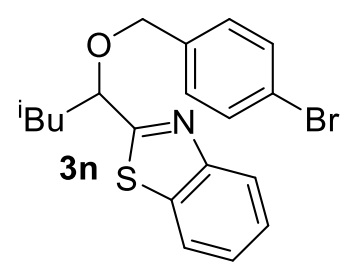

The titled compound was prepared by following the general procedure $\mathbf{C}$, obtained as a yellowish liquid (444 mg, 84\% yield). ${ }^{1} \mathbf{H}$ NMR (400 $\left.\mathbf{~ M H z , ~} \mathbf{C D C l}_{3}\right) \delta 8.02(\mathrm{~d}, J=8.24$ $\mathrm{Hz}, 1 \mathrm{H}), 7.91(\mathrm{~d}, J=8.24 \mathrm{~Hz}, 1 \mathrm{H}), 7.52-7.45(\mathrm{~m}, 3 \mathrm{H}), 7.40(\mathrm{t}, J=8.25 \mathrm{~Hz}, 1 \mathrm{H}), 7.23(\mathrm{~d}$, $J=8.70 \mathrm{~Hz}, 2 \mathrm{H}), 4.88(\mathrm{dd}, J=8.93,4.81 \mathrm{~Hz}, 1 \mathrm{H}), 4.60(\mathrm{~d}, J=11.45 \mathrm{~Hz}, 1 \mathrm{H}), 4.43(\mathrm{~d}, J=$ $11.91 \mathrm{~Hz}, 1 \mathrm{H}), 2.01-1.83(\mathrm{~m}, 2 \mathrm{H}), 1.70-1.65(\mathrm{~m}, 1 \mathrm{H}), 0.97(\mathrm{~d}, J=6.41 \mathrm{~Hz}, 3 \mathrm{H}), 0.91(\mathrm{~d}$, $J=6.41 \mathrm{~Hz}, 3 \mathrm{H}) .{ }^{13} \mathbf{C} \mathbf{N M R}\left(\mathbf{1 0 0} \mathbf{~ M H z}, \mathbf{C D C l}_{3}\right) \delta 175.5,153.0,136.5,134.9,131.5,129.6$, 126.0, 125.2, 123.0, 121.9, 121.8, 78.2, 71.2, 46.3, 24.6, 23.1, 21.9. FTIR (cm $\left.{ }^{-1}\right): 3020,2963,2875,1502,1480$, 1453, 1315, 1168, 1080, 1015, 930. HRMS(ESI): $\mathrm{m} / \mathrm{z}$ calculated for $\mathrm{C}_{19} \mathrm{H}_{21} \mathrm{ONBrS}[\mathrm{M}+\mathrm{H}]^{+}: 390.0522$, found: 390.0527.

\section{2-(1-((4-methylbenzyl)oxy)ethyl)benzo[d]thiazole (3o)}

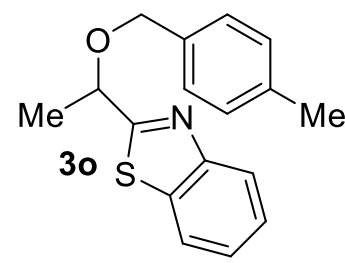

The titled compound was prepared by following the general procedure $\mathbf{C}$, obtained as a yellowish liquid (351 mg, 74\% yield). ${ }^{1} \mathbf{H}$ NMR (400 $\left.\mathbf{~ M H z}, \mathbf{C D C l}_{3}\right){ }^{1} \mathbf{H}$ NMR (400 MHz, $\left.\mathbf{C D C l}_{3}\right) \delta 8.00(\mathrm{~d}, J=8.24 \mathrm{~Hz}, 1 \mathrm{H}), 7.91(\mathrm{~d}, J=9.16 \mathrm{~Hz}, 1 \mathrm{H}), 7.48(\mathrm{~d}, J=8.24 \mathrm{~Hz}, 1 \mathrm{H})$, $7.39(\mathrm{~d}, J=7.32 \mathrm{~Hz}, 1 \mathrm{H}), 7.28(\mathrm{~d}, J=7.79 \mathrm{~Hz}, 2 \mathrm{H}), 7.17(\mathrm{~d}, J=8.24 \mathrm{~Hz}, 2 \mathrm{H}), 4.96(\mathrm{q}, J$ $=6.41 \mathrm{~Hz}, 1 \mathrm{H}), 4.64(\mathrm{~d}, J=11.45 \mathrm{~Hz}, 1 \mathrm{H}), 4.54(\mathrm{~d}, J=11.45 \mathrm{~Hz}, 1 \mathrm{H}), 2.34(\mathrm{~s}, 3 \mathrm{H}), 1.66$

$(\mathrm{d}, J=6.87 \mathrm{~Hz}, 3 \mathrm{H}){ }^{\mathbf{1 3}} \mathbf{C}$ NMR $\left(\mathbf{1 0 0} \mathbf{~ M H z}, \mathbf{C D C l}_{\mathbf{3}}\right) \delta 176.2,153.1,137.6,135.0,134.4$, 129.1, 128.0, 125.9, 125.0, 122.9, 121.9, 75.4, 71.6, 22.6, 21.2. FTIR $\left(\mathbf{c m}^{-1}\right):$ 2926, 2862, 1611, 1516, 1450, 1378, 1312, 1247, 1192, 1095, 1034, 974, 909. HRMS(ESI): $\mathrm{m} / \mathrm{z}$ calculated for $\mathrm{C}_{17} \mathrm{H}_{18} \mathrm{ONS}[\mathrm{M}+\mathrm{H}]^{+}: 284.1104$, found: 284.1105 .

\section{2-(1-((4-methylbenzyl)oxy)-2-phenylethyl)benzo[d]thiazole (3p)}

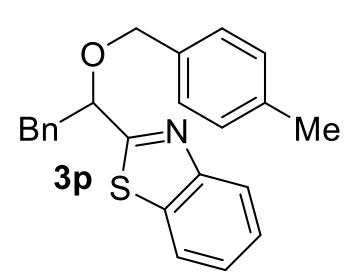

The titled compound was prepared by following the general procedure $\mathbf{C}$, obtained as a yellowish liquid (171 mg, 89\% yield). ${ }^{1} \mathbf{H}$ NMR (400 $\left.\mathbf{~ M H z , ~} \mathbf{C D C l}_{3}\right) \delta 8.01(\mathrm{~d}, J=8.13$ $\mathrm{Hz}, 1 \mathrm{H}), 7.90(\mathrm{~d}, J=8.00 \mathrm{~Hz}, 1 \mathrm{H}), 7.48(\mathrm{t}, J=8.32 \mathrm{~Hz}, 1 \mathrm{H}), 7.39$ (t, $J=8.19 \mathrm{~Hz}, 1 \mathrm{H})$, $7.29-7.20(\mathrm{~m}, 5 \mathrm{H}), 7.10-7.00(\mathrm{~m}, 4 \mathrm{H}), 5.02(\mathrm{dd}, J=8.76,4.38 \mathrm{~Hz}, 1 \mathrm{H}), 4.58(\mathrm{~d}, J=$ $11.38 \mathrm{~Hz}, 1 \mathrm{H}), 4.36$ (d, $J=11.38 \mathrm{~Hz}, 1, \mathrm{H}) 3.27$ (dd, $J=13.95,4.44 \mathrm{~Hz}, 1 \mathrm{H}), 3.2$ (dd, $J=$ 13.95, 8.69 Hz, 1H), 2.32 (s, 3H). ${ }^{\mathbf{1 3}} \mathbf{C}$ NMR (125 $\left.\mathbf{~ M H z}, \mathbf{C D C l}_{\mathbf{3}}\right) \delta 174.9,153.2,137.5$, 137.2, 135.0, 134.2, 129.7, 129.0, 128.2, 128.0, 126.6, 126.0, 125.0, 123.0, 121.9, 80.3, 72.3, 43.4, 21.1. FTIR 
$\left(\mathbf{c m}^{-1}\right): 3019,2978,2926,2865,1511,1446,1319,1216,1172,1084,1020,934$. HRMS(ESI): m/z calculated for $\mathrm{C}_{23} \mathrm{H}_{22} \mathrm{ONS}[\mathrm{M}+\mathrm{H}]^{+}: 360.1417$, found: 360.1420 .

\section{2-(1-((4-methoxybenzyl)oxy)ethyl)benzo[d]thiazole (3q)}

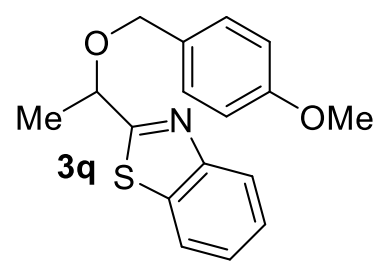

The titled compound was prepared by following the general procedure $\mathbf{C}$, obtained as a yellowish liquid (386 mg, 77\% yield). ${ }^{1} \mathbf{H}$ NMR (400 $\left.\mathbf{~ M H z , ~} \mathbf{C D C l}_{3}\right) \delta 8.00(\mathrm{~d}, J=7.63$ $\mathrm{Hz}, 1 \mathrm{H}), 7.91(\mathrm{~d}, J=7.63 \mathrm{~Hz}, 1 \mathrm{H}), 7.48(\mathrm{t}, J=8.4 \mathrm{~Hz}, 1 \mathrm{H}), 7.38(\mathrm{t}, J=8.4 \mathrm{~Hz}, 1 \mathrm{H})$, $7.31(\mathrm{~d}, J=8.39 \mathrm{~Hz}, 2 \mathrm{H}), 6.89(\mathrm{~d}, J=8.4 \mathrm{~Hz}, 2 \mathrm{H}), 4.95(\mathrm{q}, J=6.10 \mathrm{~Hz}, 1 \mathrm{H}), 4.61(\mathrm{~d}, J$ $=11.44 \mathrm{~Hz}, 1 \mathrm{H}), 4.50(\mathrm{~d}, J=11.44 \mathrm{~Hz}, 1 \mathrm{H}), 3.80(\mathrm{~s}, 3 \mathrm{H}), 1.65(\mathrm{~d}, J=6.11 \mathrm{~Hz}, 3 \mathrm{H}) .{ }^{13} \mathbf{C}$ NMR (100 MHz, $\left.\mathbf{C D C l}_{3}\right) \delta 176.2,159.4,153.1,134.9,129.6,129.6,125.9,125.0$, 122.9, 121.9, 113.9, 75.3, 71.4, 55.3, 22.6. FTIR $\left(\mathbf{c m}^{-1}\right):$ 2927, 2866, 1613, 1515, 1452, 1377, 1309, 1248, 1180, 1095, 1034, 974, 911. HRMS(ESI): $\mathrm{m} / \mathrm{z}$ calculated for $\mathrm{C}_{17} \mathrm{H}_{18} \mathrm{O}_{2} \mathrm{NS}[\mathrm{M}+\mathrm{H}]^{+}: 300.1053$ found: 300.1049 .

\section{2-(1-((3-(trifluoromethyl)benzyl)oxy)ethyl)benzo[d]thiazole (3r)}

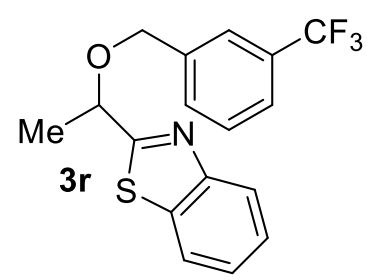

The titled compound was prepared by following the general procedure $\mathbf{C}$, obtained as a yellowish liquid (414 mg, 69\% yield). ${ }^{1} \mathbf{H}$ NMR (500 $\left.\mathbf{~ M H z , ~} \mathbf{C D C l}_{3}\right) \delta 8.01$ (d, $J=8.01$ $\mathrm{Hz}, 1 \mathrm{H}), 7.92$ (d, $J=8.01 \mathrm{~Hz}, 1 \mathrm{H}), 7.65(\mathrm{~s}, 1 \mathrm{H}), 7.56(\mathrm{~d}, J=8.01 \mathrm{~Hz}, 2 \mathrm{H}), 7.52-7.45$ $(\mathrm{m}, 2 \mathrm{H}), 7.44-7.39(\mathrm{~m}, 1 \mathrm{H}), 5.00(\mathrm{q}, J=6.61 \mathrm{~Hz}, 1 \mathrm{H}), 4.71(\mathrm{~d}, J=11.83 \mathrm{~Hz}, 1 \mathrm{H}), 4.64$ $(\mathrm{d}, J=12.21 \mathrm{~Hz}, 1 \mathrm{H}), 1.72(\mathrm{~d}, J=6.48 \mathrm{~Hz}, 3 \mathrm{H}) .{ }^{13} \mathbf{C} \mathbf{N M R}\left(\mathbf{2 5} \mathbf{M H z}, \mathbf{C D C l}_{3}\right) \delta 175.3$, 153.0, 138.7, 134.9, $131.4(\mathrm{C}-\mathrm{F}, 2 \mathrm{JC}-\mathrm{F}=32.4 \mathrm{~Hz}), 131.0(\mathrm{C}-\mathrm{F}, 2 \mathrm{JC}-\mathrm{F}=32.4 \mathrm{~Hz})$, 130.8, $130.7(\mathrm{C}-\mathrm{F}, 2 \mathrm{JC}-\mathrm{F}=32.4 \mathrm{~Hz}), 130.4(\mathrm{C}-\mathrm{F}, 2 \mathrm{JC}-\mathrm{F}=32.4 \mathrm{~Hz}), 128.9,127.3(\mathrm{C}-\mathrm{F}, 1 \mathrm{JC}-\mathrm{F}=272.8 \mathrm{~Hz})$, 126.1, 125.2, $125.1(\mathrm{C}-\mathrm{F}, 1 \mathrm{JC}-\mathrm{F}=272.8 \mathrm{~Hz}), 124.7(\mathrm{C}-\mathrm{F}, 3 \mathrm{JC}-\mathrm{F}=3.8 \mathrm{~Hz}), 124.6(\mathrm{C}-\mathrm{F}, 3 \mathrm{JC}-\mathrm{F}=3.8 \mathrm{~Hz}), 124.6$ $(\mathrm{C}-\mathrm{F}, 3 \mathrm{JC}-\mathrm{F}=3.8 \mathrm{~Hz}), 124.6(\mathrm{C}-\mathrm{F}, 3 \mathrm{JC}-\mathrm{F}=3.82 \mathrm{~Hz}), 124.4(\mathrm{C}-\mathrm{F}, 4 \mathrm{JC}-\mathrm{F}=3.8 \mathrm{~Hz}), 124.3(\mathrm{C}-\mathrm{F}, 4 \mathrm{JC}-\mathrm{F}=3.8 \mathrm{~Hz})$, $124.3(\mathrm{C}-\mathrm{F}, 4 \mathrm{JC}-\mathrm{F}=3.8 \mathrm{~Hz}), 124.3(\mathrm{C}-\mathrm{F}, 4 \mathrm{JC}-\mathrm{F}=3.8 \mathrm{~Hz}), 123.1,123.0(\mathrm{C}-\mathrm{F}, 1 \mathrm{JC}-\mathrm{F}=272.8 \mathrm{~Hz}), 122.0,120.8$ $(\mathrm{C}-\mathrm{F}, 1 \mathrm{JC}-\mathrm{F}=272.8 \mathrm{~Hz}), 76.1,70.9,22.6 .{ }^{19} \mathbf{F} \mathbf{N M R}\left(\mathbf{C D C l}_{3}, 376 \mathbf{M H z}\right) \delta-62.63(\mathrm{~s}, 3 \mathrm{~F})$. FTIR $\left(\mathbf{c m}^{-1}\right): 2982$, 2971, 2860, 1599, 1520, 1442, 1373, 1327, 1172, 1128, 1085, 924. HRMS(ESI): $\mathrm{m} / \mathrm{z}$ calculated for $\mathrm{C}_{17} \mathrm{H}_{14} \mathrm{ONF}_{3} \mathrm{NaS}[\mathrm{M}+\mathrm{Na}]^{+}: 360.0640$, found: 360.3231 .

\section{4-((1-(benzo[d]thiazol-2-yl)ethoxy)methyl)benzonitrile (3s)}

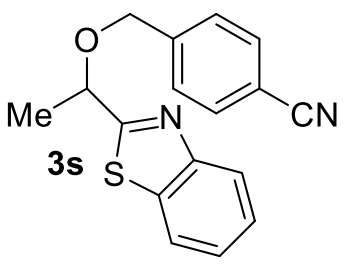

The titled compound was prepared by following the general procedure $\mathbf{C}$, obtained as a yellowish liquid (440 mg, 89\% yield). ${ }^{1} \mathbf{H}$ NMR (400 $\mathbf{~ M H z , ~} \mathbf{C D C l}_{3}$ ) $\delta 7.94$ (d, $J=8.24$ $\mathrm{Hz}, 1 \mathrm{H}), 7.84(\mathrm{~d}, J=8.70 \mathrm{~Hz}, 1 \mathrm{H}), 7.58$ (d, $J=8.70 \mathrm{~Hz}, 2 \mathrm{H}), 7.46-7.39$ (m, 3H), $7.34(\mathrm{t}, J=8.24 \mathrm{~Hz}, 1 \mathrm{H}), 4.92(\mathrm{q}, J=6.72 \mathrm{~Hz}, 1 \mathrm{H}), 4.62(\mathrm{~d}, J=12.82 \mathrm{~Hz}, 1 \mathrm{H})$, $4.56(\mathrm{~d}, J=12.82 \mathrm{~Hz}, 1 \mathrm{H}), 1.65(\mathrm{~d}, J=6.41 \mathrm{~Hz}, 3 \mathrm{H}) .{ }^{13} \mathbf{C}$ NMR $\left(\mathbf{1 0 0} \mathbf{M H z}, \mathbf{C D C l}_{3}\right) \delta$ $174.8,153.0,143.1,134.8,132.3,127.8,126.2,125.3,123.1,121.9,118.8,111.5,76.3$, 70.5, 22.5. FTIR $\left(\mathbf{c m}^{-1}\right):$ 2982, 2927, 2870, 2228, 1722, 1609, 1515, 1446, 1376, 1312, $1192,1099,978$. HRMS(ESI): $\mathrm{m} / \mathrm{z}$ calculated for $\mathrm{C}_{17} \mathrm{H}_{15} \mathrm{ON}_{2} \mathrm{~S}[\mathrm{M}+\mathrm{H}]^{+}: 295.0900$, found: 295.0895 .

\section{2-(1-(furan-2-ylmethoxy)-3-methylbutyl)benzo[d]thiazole (3t)}<smiles>BrC(Br)c1nc2ccccc2s1</smiles>

The titled compound was prepared by following the general procedure $\mathbf{C}$, obtained as a yellowish liquid (305 mg, 75\% yield). ${ }^{1} \mathbf{H}$ NMR (400 $\left.\mathbf{~ M H z}, \mathbf{C D C l}_{\mathbf{3}}\right) \delta 8.01(\mathrm{~d}, J=8.24 \mathrm{~Hz}$, 1H), $7.91(\mathrm{~d}, J=8.70 \mathrm{~Hz}, 1 \mathrm{H}), 7.48(\mathrm{t}, J=8.24 \mathrm{~Hz}, 1 \mathrm{H}), 7.43-7.33(\mathrm{~m}, 2 \mathrm{H}), 6.37-6.27(\mathrm{~m}$, 2H), $4.91(\mathrm{dd}, J=9.39,4.35 \mathrm{~Hz}, 1 \mathrm{H}), 4.62(\mathrm{~d}, J=12.82,1 \mathrm{H}), 4.45(\mathrm{~d}, J=12.82 \mathrm{~Hz}, 1 \mathrm{H}), 1.96$ - $1.80(\mathrm{~m}, 2 \mathrm{H}), 1.70-1.61(\mathrm{~m}, 1 \mathrm{H}), 0.94(\mathrm{~d}, J=6.87 \mathrm{~Hz}, 3 \mathrm{H}), 0.86(\mathrm{~d}, J=6.41 \mathrm{~Hz}, 3 \mathrm{H}) .{ }^{13} \mathrm{C}$ NMR (100 MHz, $\left.\mathbf{C D C l}_{3}\right) \delta 175.7,153.1,150.9,143.0,135.0,125.9,125.0,123.0,121.9$, 
110.3, 110.0, 77.4, 63.6, 46.3, 24.5, 23.1, 21.6. FTIR (cm $\left.{ }^{-1}\right):$ 2956, 2924, 2870, 1511, 1452, 1356, 1319, 1278, 1153, 1076, 1018, 924. HRMS(ESI): m/z calculated for $\mathrm{C}_{17} \mathrm{H}_{20} \mathrm{O}_{2} \mathrm{NS}[\mathrm{M}+\mathrm{H}]^{+}:$302.1209, found: 302.1211 .

\section{2-(3-methyl-1-(thiophen-2-ylmethoxy)butyl)benzo[d]thiazole (3u)}<smiles>CC(C)C(COCc1cccs1)c1nc2ccccc2s1</smiles>

The titled compound was prepared by following the general procedure $\mathbf{C}$, obtained as a yellowish liquid (403 mg, 93\% yield). ${ }^{1} \mathbf{H}$ NMR (400 $\left.\mathbf{M H z}, \mathbf{C D C l}_{3}\right) \delta 8.02(\mathrm{~d}, J=8.13 \mathrm{~Hz}$, $1 \mathrm{H}), 7.90(\mathrm{~d}, J=8.50 \mathrm{~Hz}, 1 \mathrm{H}), 7.48(\mathrm{t}, J=8.24 \mathrm{~Hz}, 1 \mathrm{H}), 7.38(\mathrm{t}, J=8.24 \mathrm{~Hz}, 1 \mathrm{H}), 7.29$ (d, $J=$ $6.25 \mathrm{~Hz}, 1 \mathrm{H}), 7.00(\mathrm{~d}, J=2.63 \mathrm{~Hz}, 1 \mathrm{H}), 6.96(\mathrm{dd}, J=5.00,3.50 \mathrm{~Hz}, 1 \mathrm{H}), 4.93(\mathrm{dd}, J=9.07$, $4.57 \mathrm{~Hz}, 1 \mathrm{H}), 4.82(\mathrm{~d}, J=12.26 \mathrm{~Hz}, 1 \mathrm{H}), 4.65(\mathrm{~d}, J=12.38 \mathrm{~Hz}, 1 \mathrm{H}), 1.98-1.82(\mathrm{~m}, 2 \mathrm{H}), 1.71$ - $1.62(\mathrm{~m}, 1 \mathrm{H}), 0.95(\mathrm{~d}, J=6.50 \mathrm{~Hz}, 3 \mathrm{H}), 0.87(\mathrm{~d}, J=6.50 \mathrm{~Hz}, 3 \mathrm{H}) .{ }^{13} \mathbf{C}$ NMR $(\mathbf{1 0 0} \mathbf{M H z}$, $\left.\mathbf{C D C l}_{3}\right) \delta 175.5,153.1,139.9,135.0,127.1,126.6,126.2,125.9,125.1,123.0,121.9,77.4,66.2,46.2,24.5,23.1$, 21.7. FTIR $\left(\mathbf{c m}^{-1}\right): 2954,2870,1516,1450,1376,1355,1317,1275,1174,1077,1021,937$. HRMS(ESI): m/z calculated for $\mathrm{C}_{17} \mathrm{H}_{20} \mathrm{ONS}_{2}[\mathrm{M}+\mathrm{H}]^{+}: 318.0981$, found: 318.0983 .

\section{2-((1-phenylethoxy)methyl)benzo[d]thiazole (S11)}<smiles>CC(OCc1nc2ccccc2s1)c1ccccc1</smiles>

The titled compound was prepared by following the general procedure $\mathbf{D}-\mathbf{4}$, obtained as a yellowish liquid (539 mg, 64\% yield). ${ }^{1}$ HNMR (500 $\left.\mathbf{~ M H z , ~} \mathbf{C D C l}_{3}\right) \delta 7.97(\mathrm{~d}, J=8.01 \mathrm{~Hz}, 1 \mathrm{H})$, $7.90(\mathrm{~d}, J=8.01 \mathrm{~Hz}, 1 \mathrm{H}), 7.46(\mathrm{t}, J=7.63 \mathrm{~Hz}, 1 \mathrm{H}), 7.42-7.34(\mathrm{~m}, 5 \mathrm{H}), 7.34-7.27(\mathrm{~m}, 1 \mathrm{H})$, $4.80(\mathrm{~d}, J=14.11 \mathrm{~Hz}, 1 \mathrm{H}), 4.74(\mathrm{~d}, J=14.11 \mathrm{~Hz}, 1 \mathrm{H}), 4.65(\mathrm{q}, J=6.49 \mathrm{~Hz}, 1 \mathrm{H}), 1.57(\mathrm{~d}, J=$ $6.49 \mathrm{~Hz}, 3 \mathrm{H}),{ }^{13} \mathbf{C}$ NMR $\left(\mathbf{1 2 5} \mathbf{~ M H z}, \mathbf{C D C l}_{3}\right) \delta 170.9,153.2,142.4,135.0,128.6,127.9,126.3$, 125.9, 124.9, 122.9, 121.7, 78.7, 67.9, 23.8. FTIR $\left(\mathbf{c m}^{-1}\right): 3026,2974,2924,2860,1524,1445$, 1316, 1273, 1173, 1097, 1017. HRMS(ESI): $\mathrm{m} / \mathrm{z}$ calculated for $\mathrm{C}_{16} \mathrm{H}_{16} \mathrm{ONS}[\mathrm{M}+\mathrm{H}]^{+}: 270.0947$, found: 270.0947.

\section{2-(1-(1-phenylethoxy)ethyl)benzo[d]thiazole (3v)}

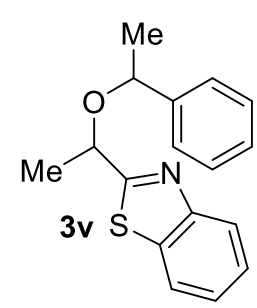

The titled compound was prepared by following the general procedure $\mathbf{D - 5}$, major product was isolated via column (186 mg, 66\% yield). ${ }^{\mathbf{1}} \mathbf{H}$ NMR (400 $\mathbf{~ M H z , ~} \mathbf{C D C l}_{\mathbf{3}}$ ) Major Isomer $\delta 7.98$ $(\mathrm{d}, J=7.63 \mathrm{~Hz}, 1 \mathrm{H}), 7.93(\mathrm{~d}, J=7.63 \mathrm{~Hz}, 1 \mathrm{H}), 7.48(\mathrm{t}, J=7.63 \mathrm{~Hz}, 1 \mathrm{H}), 7.42-7.28(\mathrm{~m}, 6 \mathrm{H})$, $4.75(\mathrm{q}, J=6.36 \mathrm{~Hz}, 1 \mathrm{H}), 4.55(\mathrm{q}, J=6.87 \mathrm{~Hz}, 1 \mathrm{H}), 1.55(\mathrm{~d}, J=6.87 \mathrm{~Hz}, 3 \mathrm{H}), 1.50(\mathrm{~d}, J=6.87$ $\mathrm{Hz}, 3 \mathrm{H}) .{ }^{13} \mathbf{C}$ NMR (100 MHz, $\left.\mathbf{C D C l}_{3}\right) \delta 177.1,153.3,142.9,134.8,128.6,127.8,126.3,125.9$, 125.0, 122.9, 121.9, 77.2, 73.6, 24.4, 23.4. FTIR $\left(\mathbf{c m}^{-1}\right):$ 2973, 2925, 2864, 1518, 1446, 1370, 1308, 1086, 1021, 936. HRMS(ESI): $\mathrm{m} / \mathrm{z}$ calculated for $\mathrm{C}_{17} \mathrm{H}_{18} \mathrm{ONS}[\mathrm{M}+\mathrm{H}]^{+}: 284.1104$, found:

284.1101 .

\section{2-((1-(naphthalen-2-yl)ethoxy)methyl)benzo[d]thiazole (S12)}

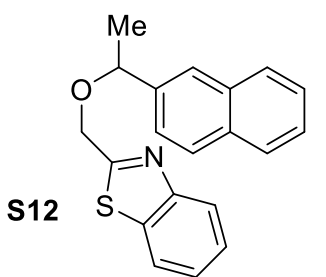

The titled compound was prepared by following the general procedure $\mathbf{D - 4}$, obtained as a yellowish liquid (252 mg, 69\% yield). ${ }^{1} \mathbf{H}$ NMR (400 MHz, $\left.\mathbf{C D C l}_{3}\right) \delta 7.96(\mathrm{~d}, J=8.24 \mathrm{~Hz}$, $1 \mathrm{H}), 7.90(\mathrm{~d}, J=7.33 \mathrm{~Hz}, 1 \mathrm{H}), 7.89-7.81(\mathrm{~m}, 3 \mathrm{H}), 7.80(\mathrm{~s}, 1 \mathrm{H}), 7.54(\mathrm{dd}, J=8.70,1.83 \mathrm{~Hz}$, 1H), $7.52-7.43(\mathrm{~m}, 3 \mathrm{H}), 7.41-7.35(\mathrm{~m}, 1 \mathrm{H}), 4.86-4.76(\mathrm{~m}, 3 \mathrm{H}), 1.64(\mathrm{~d}, J=6.41 \mathrm{~Hz}, 3 \mathrm{H})$. ${ }^{13} \mathbf{C}$ NMR (100 MHz, $\left.\mathbf{C D C l}_{3}\right) \delta 170.9,153.0,139.7,134.9,133.2,128.7,127.9,127.7$, 126.2, 126.0, 125.5, 125.0, 124.0, 122.9, 121.7, 78.8, 67.9, 23.8. FTIR $\left(\mathbf{c m}^{-1}\right): 2972,2923,2856,1519,1443$, 1366, 1315, 1242, 1172, 1094, 1009, 952. HRMS(ESI): $\mathrm{m} / \mathrm{z}$ calculated for $\mathrm{C}_{20} \mathrm{H}_{18} \mathrm{ONS}[\mathrm{M}+\mathrm{H}]^{+}: 320.1104$, found: 320.1093 . 


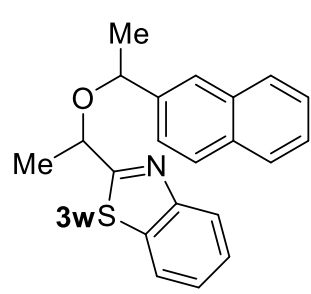

The titled compound was prepared by following the general procedure $\mathbf{D - 5}$, and the major product obtained as a yellowish liquid (132 mg, 63\% yield). ${ }^{1} \mathbf{H}$ NMR (400 $\mathbf{~ M H z}, \mathbf{C D C l}_{\mathbf{3}}$ ) Major Isomer $\delta 8.01-7.92(\mathrm{~m}, 2 \mathrm{H}), 7.90-7.80(\mathrm{~m}, 3 \mathrm{H}), 7.73(\mathrm{~s}, 1 \mathrm{H}), 7.55-7.45(\mathrm{~m}, 4 \mathrm{H})$, $7.43-7.38(\mathrm{~m}, 1 \mathrm{H}), 4.79(\mathrm{q}, J=6.63 \mathrm{~Hz}, 1 \mathrm{H}), 4.72(\mathrm{q}, J=6.50 \mathrm{~Hz}, 1 \mathrm{H}), 1.59(\mathrm{~d}, J=6.50$ $\mathrm{Hz}, 3 \mathrm{H}), 1.55(\mathrm{~d}, J=6.63 \mathrm{~Hz}, 3 \mathrm{H}) .{ }^{13} \mathbf{C} \mathbf{~ N M R}\left(\mathbf{1 0 0} \mathbf{~ M H z}, \mathbf{C D C l}_{3}\right) \delta 177.0,153.4,140.3$, $134.9,133.2$, 133.2, 128.6, 127.9, 127.7, 126.2, 125.9, 125.7, 125.0, 124.0, 123.0, 121.9, 77.4, 73.7, 24.2, 23.4. FTIR (cm $\left.{ }^{-1}\right):$ 2979, 2926, 2896, 1595, 1516, 1489, 1445, 1371, 1307 , 1197, 1093, 1018, 939. HRMS(ESI): m/z calculated for $\mathrm{C}_{21} \mathrm{H}_{20} \mathrm{ONS}[\mathrm{M}+\mathrm{H}]^{+}: 334.1260$, found: 334.1257 .

\section{2-((cyclopropyl(phenyl)methoxy)methyl)benzo[d]thiazole (S13)}<smiles>c1ccc(C(OCc2nc3ccccc3s2)C2CC2)cc1</smiles>

The titled compound was prepared by following the general procedure D-4, obtained as a yellowish liquid (769 mg, 62\% yield). ${ }^{1} \mathbf{H}$ NMR (400 $\left.\mathbf{M H z}, \mathbf{C D C l}_{\mathbf{3}}\right) \delta 7.96(\mathrm{~d}, J=8.39 \mathrm{~Hz}, 1 \mathrm{H})$, $7.90(\mathrm{~d}, J=7.63 \mathrm{~Hz}, 1 \mathrm{H}), 7.48-7.43(\mathrm{~m}, 1 \mathrm{H}), 7.42-7.28(\mathrm{~m}, 6 \mathrm{H}), 4.84(\mathrm{~d}, J=14.50 \mathrm{~Hz}, 1 \mathrm{H})$, $4.79(\mathrm{~d}, J=13.73 \mathrm{~Hz}, 1 \mathrm{H}), 3.91(\mathrm{~d}, J=7.63 \mathrm{~Hz}, 1 \mathrm{H}), 1.31-1.24(\mathrm{~m}, 1 \mathrm{H}), 0.75-0.67(\mathrm{~m}, 1 \mathrm{H})$, $0.63-0.56(\mathrm{~m}, 1 \mathrm{H}), 0.55-0.47(\mathrm{~m}, 1 \mathrm{H}), 0.35-0.29(\mathrm{~m} \mathrm{1H}) .{ }^{\mathbf{1 3}} \mathbf{C}$ NMR $\left(\mathbf{1 0 0} \mathbf{~ M H z}, \mathbf{C D C l}_{\mathbf{3}}\right) \delta$ $170.9,153.0,140.8,135.0,128.5,127.9,126.9,125.9,124.9,122.9,121.7,86.0,67.8,17.4,4.4$, 2.1. FTIR $\left(\mathbf{c m}^{-1}\right):$ 3013, 2923, 2860, 1523, 1444, 1375, 1309, 1245, 1169, 1093, 1017, 912. HRMS(ESI): $\mathrm{m} / \mathrm{z}$ calculated for $\mathrm{C}_{18} \mathrm{H}_{18} \mathrm{ONS}[\mathrm{M}+\mathrm{H}]^{+}: 296.1104$, found: 296.1093 .

\section{2-(1-(cyclopropyl(phenyl)methoxy)ethyl)benzo[d]thiazole (3x)}

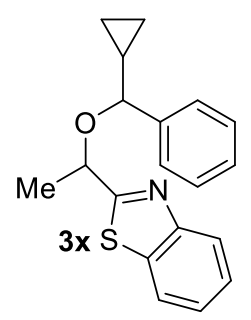

The titled compound was prepared by following the general procedure $\mathbf{D - 5}$, and the major product obtained as a yellowish liquid (166 mg, 64\% yield). ${ }^{1} \mathbf{H}$ NMR (400 $\mathbf{~ M H z}, \mathbf{C D C l}_{3}$ ) Major Isomer $\delta 7.98-7.91(\mathrm{~m}, 2 \mathrm{H}), 7.50-7.45(\mathrm{~m}, 1 \mathrm{H}), 7.42-7.31(\mathrm{~m}, 6 \mathrm{H}), 4.75(\mathrm{q}, J=6.41 \mathrm{~Hz}, 1 \mathrm{H})$, $3.77(\mathrm{~d}, J=7.78 \mathrm{~Hz}, 1 \mathrm{H}), 1.56(\mathrm{~d}, J=6.41 \mathrm{~Hz}, 3 \mathrm{H}), 1.30-1.22(\mathrm{~m}, 1 \mathrm{H}), 0.71-0.64(\mathrm{~m}, 1 \mathrm{H})$, $0.57-0.49(\mathrm{~m}, 1 \mathrm{H}), 0.49-0.47(\mathrm{~m}, 1 \mathrm{H}), 0.28-0.21(\mathrm{~m}, 1 \mathrm{H}) .{ }^{\mathbf{1 3}} \mathbf{C} \mathbf{~ N M R}\left(\mathbf{1 0 0} \mathbf{~ M H z}, \mathbf{C D C l}_{\mathbf{3}}\right) \delta$ $176.7,153.1,141.2,134.9,128.5,127.9,126.9,125.9,125.0,122.9,121.9,85.0,73.4,23.4,18.2$, 4.5, 2.5, FTIR $\left(\mathbf{c m}^{-1}\right):$ 2994, 2977, 2923, 2866, 1516, 1446, 1372, 1307, 1188, 1086, 1032, 975 , 901. HRMS(ESI): $\mathrm{m} / \mathrm{z}$ calculated for $\mathrm{C}_{19} \mathrm{H}_{20} \mathrm{ONS}[\mathrm{M}+\mathrm{H}]^{+}: 310.1260$, found: 310.1265 .

\section{2-(1-(benzhydryloxy)-3-methylbutyl)benzo[d]thiazole (3y)}

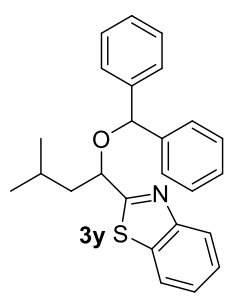

In an oven dried $50 \mathrm{ml}$ round bottom flask with a magnetic bar, alcohol $(1.5 \mathrm{~g}, 6.787 \mathrm{mmol}$, 1.0 equiv.), p-Toluenesulfonic acid (1.357 mmol, 0.2 equiv.), diphenylmethanol ( $3.1 \mathrm{~g}$, $16.968 \mathrm{mmol})$ was taken in dry Toluene $(34 \mathrm{ml})$ at room temperature, sealed by insulating tape and made oxygen - free via degassing for $10 \mathrm{~min}$. The reaction container was heated to $80{ }^{\circ} \mathrm{C}$ in a oil bath for overnight and quenched with saturated $\mathrm{NaHCO}_{3}$ solution. The organic layer was extracted with EtOAc (3 times) and combined organic layer was dried over anhydrous $\mathrm{Na}_{2} \mathrm{SO}_{4}$. The solvent removal under reduced pressure gave the crude product which was further purified by column chromatography on silica gel using ethylacetate-pet ether $(1.5-2 \% \mathrm{EA} / \mathrm{PE})$ as eluent to obtain the desired product with (1.3g, 43\% yield). ${ }^{1}$ HNMR $\left(200 \mathbf{~ M H z}, \mathbf{C D C l}_{3}\right) \delta 8.02-7.98(\mathrm{~m}, 1 \mathrm{H}), 7.93-7.78(\mathrm{~m}, 1 \mathrm{H}), 7.52$ $7.20(\mathrm{~m}, 12 \mathrm{H}), 5.47(\mathrm{~s}, 1 \mathrm{H}), 4.88(\mathrm{dd}, J=8.84,4.42 \mathrm{~Hz}, 1 \mathrm{H}), 2.10-1.77(\mathrm{~m}, 2 \mathrm{H}), 1.74-1.55(\mathrm{~m}, 1 \mathrm{H}), 0.90(\mathrm{~d}, J$ $=6.44 \mathrm{~Hz}, 3 \mathrm{H}), 0.67(\mathrm{~d}, J=6.44 \mathrm{~Hz}, 3 \mathrm{H}) .{ }^{13} \mathbf{C}$ NMR $\left(\mathbf{5 0} \mathbf{~ M H z}, \mathbf{C D C l}_{3}\right) \delta 176.1,153.1,142.1,140.9,135.0$, 128.5, 128.2, 128.1, 127.2, 126.7, 125.9, 125.0, 123.0, 121.9, 81.7, 75.5, 46.7, 24.3, 23.3, 21.6. FTIR (cm $\left.{ }^{-1}\right):$ 3021, 2967, 2875, 1641, 1495, 1451, 1307, 1268, 1058, 923. HRMS(ESI): m/z calculated for $\mathrm{C}_{25} \mathrm{H}_{26} \mathrm{ONS}$ $[\mathrm{M}+\mathrm{H}]^{+}:$388.1730, found: 388.1729 . 


\section{Characterization of salts:}

\section{2-(1-(benzyloxy)ethyl)-3-methylbenzo[d]thiazol-3-iumtrifluoromethanesulfonate (4b)}

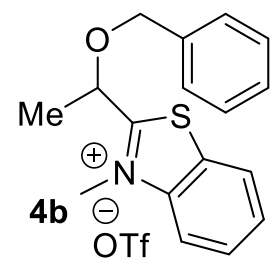

The titled compound was prepared by following the general procedure $\mathbf{E}$, obtained as a white solid (137 mg, 92\% yield). MP $118-120{ }^{\circ} \mathrm{C} .{ }^{\mathbf{1}} \mathbf{H}$ NMR (400 $\left.\mathbf{~ M H z}, \mathbf{C D C l}_{3}\right) \delta 8.08$ (d, J=4.12 $\mathrm{Hz}, 1 \mathrm{H}), 8.06(\mathrm{~d}, J=3.21 \mathrm{~Hz}, 1 \mathrm{H}), 7.83(\mathrm{t}, J=7.56 \mathrm{~Hz}, 1 \mathrm{H}), 7.76-7.68(\mathrm{~m}, 1 \mathrm{H}), 7.43-7.28$ $(\mathrm{m}, 5 \mathrm{H}), 5.60(\mathrm{q}, J=6.41 \mathrm{~Hz}, 1 \mathrm{H}), 4.82(\mathrm{~s}, 2 \mathrm{H}), 4.30(\mathrm{~s}, 3 \mathrm{H}), 1.73(\mathrm{~d}, J=6.41 \mathrm{~Hz}, 3 \mathrm{H}) .{ }^{13} \mathbf{C}$ NMR (100 MHz, $\left.\mathbf{C D C l}_{3}\right) \delta 184.8,143.0,136.3,130.2,128.9,128.9,128.6,128.4,128.2$, 123.6, 116.8, 73.2, 72.9, 37.6, 21.3. HRMS(ESI): $\mathrm{m} / \mathrm{z}$ calculated for $\mathrm{C}_{17} \mathrm{H}_{18} \mathrm{ONS}[\mathrm{M}]^{+}$: 284.1104, found: 284.1103 .

\section{2-(1-((4-cyanobenzyl)oxy)ethyl)-3-methylbenzo[d]thiazol-3-iumtrifluoromethanesulfonate (4s)}

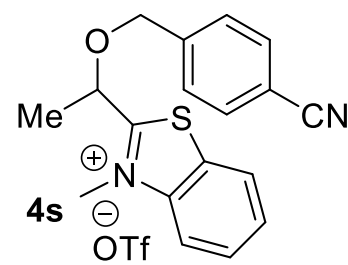

The titled compound was prepared by following the general procedure $\mathbf{E}$, obtained as a viscous liquid (141 mg, 83\% yield). ${ }^{1} \mathbf{H}$ NMR (400 $\left.\mathbf{~ M H z , ~} \mathbf{C D C l}_{3}\right) \delta 8.12$ - 8.02 (m, 2H), 7.85 (t, $J=7.33 \mathrm{~Hz}, 1 \mathrm{H}), 7.75(\mathrm{~d}, J=7.33 \mathrm{~Hz}, 1 \mathrm{H}), 7.64(\mathrm{~d}, J=8.24 \mathrm{~Hz}, 2 \mathrm{H}), 7.54(\mathrm{~d}, J=$ $8.70 \mathrm{~Hz}, 2 \mathrm{H}), 5.73(\mathrm{q}, J=6.41 \mathrm{~Hz}, 1 \mathrm{H}), 4.95(\mathrm{~s}, 2 \mathrm{H}), 4.34(\mathrm{~s}, 3 \mathrm{H}), 1.76(\mathrm{~d}, J=6.87 \mathrm{~Hz}$, 3H). ${ }^{13} \mathbf{C}$ NMR (100 MHz, $\left.\mathbf{C D C l}_{3}\right) \delta 184.7,143.0,141.9,132.3,130.3,129.0,128.8$, 128.2, 123.8, 118.7, 116.7, 111.8, 73.5, 72.0, 37.7, 21.4. FTIR $\left(\mathbf{c m}^{-1}\right): 3019,2933,2230$, $1525,1458,1342,1270,1161,1104,1032,975 . \mathbf{H R M S}(\mathrm{ESI}): \mathrm{m} / \mathrm{z}$ calculated for $\mathrm{C}_{18} \mathrm{H}_{17} \mathrm{ON}_{2} \mathrm{~S}[\mathrm{M}]^{+}: 309.1056$, found: 309.1053

\section{Characterization of $[1,3]$ products:}

\section{1-(2-benzyl-3-methyl-2,3-dihydrobenzo[d]thiazol-2-yl)-3-methylbutan-1-one (6a)}

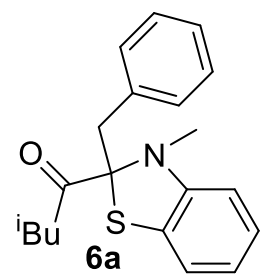

The titled compound was prepared by following the general procedure $\mathbf{F}$, obtained as a yellowish liquid (60 mg, 92\% yield). ${ }^{1} \mathbf{H}$ NMR (500 $\left.\mathbf{~ M H z , ~} \mathbf{C D C l}_{\mathbf{3}}\right) \delta 7.29-7.23(\mathrm{~m}, 2 \mathrm{H}), 7.22$ - $7.13(\mathrm{~m}, 3 \mathrm{H})$, ), $6.91(\mathrm{t}, J=7.25 \mathrm{~Hz}, 1 \mathrm{H}), 6.84(\mathrm{~d}, J=8.39 \mathrm{~Hz}, 1 \mathrm{H}), 6.55$ (t, $J=7.44 \mathrm{~Hz}$, 1H) $6.16(\mathrm{~d}, J=8.01 \mathrm{~Hz}, 1 \mathrm{H}), 3.49(\mathrm{~d}, J=14.50 \mathrm{~Hz}, 1 \mathrm{H}), 3.28(\mathrm{~d}, J=14.50 \mathrm{~Hz}, 1 \mathrm{H}), 2.87$ $2.76(\mathrm{~m}, 4 \mathrm{H}), 2.42-2.32(\mathrm{~m}, 1 \mathrm{H}), 2.28-2.16(\mathrm{~m}, 1 \mathrm{H}), 0.89-0.88(\mathrm{~m}, 6 \mathrm{H}) .{ }^{13} \mathbf{C}$ NMR (125 MHz, $\left.\mathbf{C D C l}_{3}\right) \delta 204.2$ 147.6, 135.7, 131.0, 127.8, 126.7, 125.8, 123.2, 121.0, 118.2, 105.7,

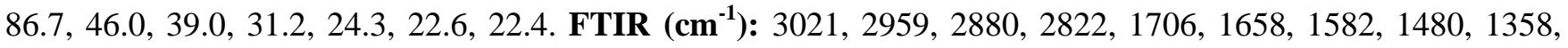
1296, 1118, 1034. HRMS(ESI): $\mathrm{m} / \mathrm{z}$ calculated for $\mathrm{C}_{20} \mathrm{H}_{24} \mathrm{ONS}[\mathrm{M}+\mathrm{H}]^{+}: 326.1573$, found: 326.1571 .

\section{1-(2-benzyl-3-methyl-2,3-dihydrobenzo[d]thiazol-2-yl)ethan-1-one (6b)}

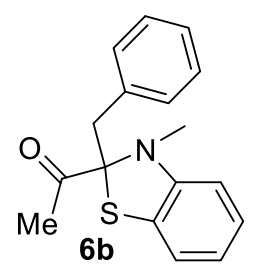

The titled compound was prepared by following the general procedure $\mathbf{F}$, obtained as a yellowish liquid (52 mg, 92\% yield). 2.5 mmol scale reaction: In a flame dried $25 \mathrm{ml}$ round bottom flask with a magnetic bar, ether $3 \mathrm{~b}$ (673 mg, 1 equiv.) and $\mathrm{K}_{2} \mathrm{CO}_{3}(518 \mathrm{mg}, 1.5$ equiv.) in dry DCM $(2.5 \mathrm{ml}, 1 \mathrm{M})$ were taken and sealed with electrical tape and degassed for $10 \mathrm{~min}$. The reaction container was cooled to $0{ }^{\circ} \mathrm{C}$ under argon atmosphere. To the solution MeOTf (1.25 equiv.) was added dropwise and sealed with insulating tape, followed by slow warming to room temperature with continued stirring for $12 \mathrm{~h}$. Next, DMSO $(10 \mathrm{~mL}, 0.25 \mathrm{M})$ was added to the reaction at room temperature and purged with argon for $10 \mathrm{~min}$ to make the reaction mixture oxygen-free and sealed with electrical tape. The reaction mixture was shifted to $50{ }^{\circ} \mathrm{C}$ oil bath with continued stirring for $12 \mathrm{~h}$. The reaction mixture was quenched with saturated $\mathrm{NH}_{4} \mathrm{Cl}$ solution at room temperature and organic layer was extracted with EtOAc (3 times). The combined organic layer was dried over anhydrous $\mathrm{Na}_{2} \mathrm{SO}_{4}$. Solvent removal under reduced pressure gave the crude product which was further purified by column chromatography on silica gel using $1-2 \%$ EtOAc-pet ether as eluent to obtain the pure product (609 mg, 86\%). 
${ }^{1}$ H NMR (500 MHz, CDCl $)$ : $\delta 7.30-7.24(\mathrm{~m}, 2 \mathrm{H}), 7.24-7.14(\mathrm{~m}, 3 \mathrm{H}), 6.91(\mathrm{t}, J=8.20 \mathrm{~Hz}, 1 \mathrm{H}), 6.84(\mathrm{~d}, J=$ $7.25 \mathrm{~Hz}, 1 \mathrm{H}), 6.84(\mathrm{~d}, J=7.25 \mathrm{~Hz}, 1 \mathrm{H}), 6.55(\mathrm{t}, J=7.63 \mathrm{~Hz}, 1 \mathrm{H}), 6.18(\mathrm{~d}, J=7.63 \mathrm{~Hz}, 1 \mathrm{H}), 3.45(\mathrm{~d}, J=14.88$ $\mathrm{Hz}, 1 \mathrm{H}), 3.29$ (d, $J=14.88 \mathrm{~Hz}, 1 \mathrm{H}), 2.80(\mathrm{~s}, 3 \mathrm{H}), 2.34$ (s, 3H). ${ }^{13} \mathbf{C}$ NMR (125 MHz, $\left.\mathbf{C D C l}_{\mathbf{3}}\right) \delta$ 203.0, 147.7, 135.7, 131.0, 127.9, 126.8, 126.0, 123.4, 121.1, 118.4, 105.9, 86.5, 38.8, 31.2, 25.3.. FTIR $\left(\mathbf{c m}^{-1}\right):$ 3007, 2923, 2819, 1705, 1661, 1583, 1478, 1355, 1301, 1186, 1120, 1080, 1026, 911. HRMS(ESI): m/z calculated for $\mathrm{C}_{17} \mathrm{H}_{18} \mathrm{ONS}[\mathrm{M}+\mathrm{H}]^{+}: 284.1104$, found: 284.1100 .

\section{1-(2-benzyl-3-methyl-2,3-dihydrobenzo[d]thiazol-2-yl)-2,2-dimethylpropan-1-one (6c)}

The titled compound was prepared by following the general procedure $\mathbf{F}$, obtained as a yellowish liquid (54 $\mathrm{mg}$, 83\% yield). ${ }^{1} \mathbf{H}$ NMR (400 MHz, $\left.\mathbf{C D C l}_{\mathbf{3}}\right) \delta 7.31-7.12(\mathrm{~m}, 5 \mathrm{H}), 6.89(\mathrm{t}, J=7.63 \mathrm{~Hz}, 1 \mathrm{H}), 6.83$ (d, $J=7.32 \mathrm{~Hz}$,

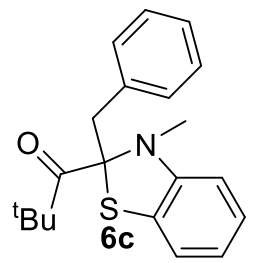
$1 \mathrm{H}), 6.53(\mathrm{t}, J=7.32 \mathrm{~Hz}, 1 \mathrm{H}), 6.12(\mathrm{~d}, J=7.32 \mathrm{~Hz}, 1 \mathrm{H}), 3.52(\mathrm{~d}, J=14.04 \mathrm{~Hz}, 1 \mathrm{H}), 3.24(\mathrm{~d}, J$ $=14.65 \mathrm{~Hz}, 1 \mathrm{H}), 2.83(\mathrm{~s}, 3 \mathrm{H}), 1.29(\mathrm{~s}, 9 \mathrm{H}) .{ }^{\mathbf{1 3}} \mathbf{C} \mathbf{N M R}\left(\mathbf{1 0 0 M H z}, \mathbf{C D C l}_{\mathbf{3}}\right) \delta 208.4,146.9$, 135.4, 131.4, 127.6, 126.7, 125.8, 123.2, 120.7,117.9, 105.6, 86.4, 44.8, 41.8, 31.5, 29.7, 28.5. FTIR $\left(\mathbf{c m}^{-1}\right):$ 3018, 2961, 2924, 2864, 1688, 1583, 1480, 1362, 1298, 1116, 1033, 952, 901. HRMS(ESI): $\mathrm{m} / \mathrm{z}$ calculated for $\mathrm{C}_{20} \mathrm{H}_{24} \mathrm{ONS}[\mathrm{M}+\mathrm{H}]^{+}: 326.1573$, found: 326.1570 .

1-(2-benzyl-3-methyl-2,3-dihydrobenzo[d]thiazol-2-yl)-2-phenylethan-1-one (6d)

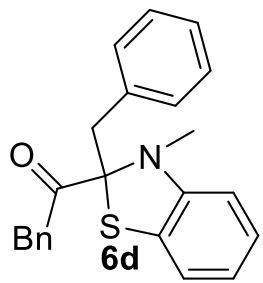

The titled compound was prepared by following the general procedure $\mathbf{F}$, obtained as a yellowish liquid (55 mg, 76\% yield). ${ }^{1} \mathbf{H}$ NMR (400 $\left.\mathbf{~ M H z , ~} \mathbf{C D C l}_{\mathbf{3}}\right) \delta 7.34-7.09$ (m, 10H), $6.97-6.85(\mathrm{~m}, 2 \mathrm{H}), 6.62-6.54(\mathrm{~m}, 1 \mathrm{H}), 6.19(\mathrm{~d}, J=7.63 \mathrm{~Hz}, 1 \mathrm{H}), 4.16(\mathrm{~d}, J=16.78 \mathrm{~Hz}, 1 \mathrm{H})$, $3.89(\mathrm{~d}, J=16.78 \mathrm{~Hz}, 1 \mathrm{H}), 3.50(\mathrm{~d}, J=14.50 \mathrm{~Hz}, 1 \mathrm{H}), 3.30(\mathrm{~d}, J=14.50 \mathrm{~Hz}, 1 \mathrm{H}), 2.73(\mathrm{~s}$, 3H). ${ }^{13} \mathbf{C}$ NMR (100 MHz, $\left.\mathbf{C D C l}_{3}\right) \delta 201.8,147.6,135.5,134.4,131.1,129.6,128.4,127.9$, 126.8, 126.8, 126.1, 123.2, 121.2, 118.5, 106.0, 86.9, 43.7, 39.0, 31.2. FTIR (cm $\left.{ }^{-1}\right): 3021,2924,2822,1713$, 1581, 1482, 1357, 1301, 1120, 1078, 1028, 977. HRMS(ESI): $\mathrm{m} / \mathrm{z}$ calculated for $\mathrm{C}_{23} \mathrm{H}_{22} \mathrm{ONS}[\mathrm{M}+\mathrm{H}]^{+}: 360.1417$, found: 360.1417 .

\section{1-(2-benzyl-3-methyl-2,3-dihydrobenzo[d]thiazol-2-yl)hexan-1-one (6e)}

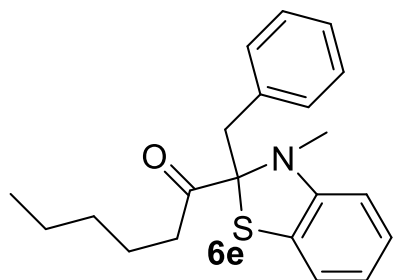

The titled compound was prepared by following the general procedure $\mathbf{F}$, obtained as a yellowish liquid (51 mg, 76\% yield). ${ }^{1} \mathbf{H}$ NMR (400 $\left.\mathbf{~ M H z , ~} \mathbf{C D C l}_{\mathbf{3}}\right) \delta 7.28-7.16$ $(\mathrm{m}, 5 \mathrm{H}), 6.92(\mathrm{t}, J=7.78 \mathrm{~Hz}, 1 \mathrm{H}), 6.84(\mathrm{~d}, J=7.79 \mathrm{~Hz}, 1 \mathrm{H}), 6.55(\mathrm{t}, J=8.01 \mathrm{~Hz}$, $1 \mathrm{H}), 6.17(\mathrm{~d}, J=8.24 \mathrm{~Hz}, 1 \mathrm{H}), 3.49(\mathrm{~d}, J=14.65 \mathrm{~Hz}, 1 \mathrm{H}), 3.28(\mathrm{~d}, J=14.65 \mathrm{~Hz}$, $1 \mathrm{H}), 2.96-2.86(\mathrm{~m}, 1 \mathrm{H}), 2.80(\mathrm{~s}, 3 \mathrm{H}), 2.50-2.41(\mathrm{~m}, 1 \mathrm{H}), 1.67-1.52(\mathrm{~m}, 2 \mathrm{H}), 1.33$ - $1.17(\mathrm{~m}, 4 \mathrm{H}), 0.85(\mathrm{t}, J=7.10 \mathrm{~Hz}, 3 \mathrm{H}) .{ }^{\mathbf{1 3}} \mathbf{C} \mathbf{~ N M R}\left(\mathbf{1 0 0} \mathbf{~ M H z}, \mathbf{C D C l}_{\mathbf{3}}\right) \delta 205.4$, 147.7, 135.8, 131.1, 127.9, 126.9, 126.0,123.4, 121.2, 118.4, 105.9, 86.8, 39.1, 37.3, 31.4, 31.4, 24.0, 22.6, 14.0. HRMS(ESI): $\mathrm{m} / \mathrm{z}$ calculated for $\mathrm{C}_{21} \mathrm{H}_{26} \mathrm{ONS}[\mathrm{M}+\mathrm{H}]^{+}: 340.1730$, found: 340.1726 .

\section{(2-benzyl-3-methyl-2,3-dihydrobenzo[d]thiazol-2-yl)(cyclohexyl)methanone (6f)}

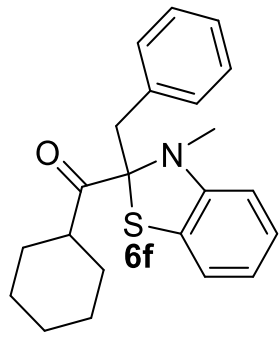

The titled compound was prepared by following the general procedure $\mathbf{F}$, obtained as a yellowish liquid (61mg, 86\% yield). ${ }^{1} \mathbf{H}$ NMR (500 $\left.\mathbf{~ M H z , ~} \mathbf{C D C l}_{3}\right) \delta 7.13-7.27(\mathrm{~m}, 5 \mathrm{H})$, $6.92(\mathrm{t}, J=7.63 \mathrm{~Hz}, 1 \mathrm{H}), 6.86(\mathrm{~d}, J=7.25 \mathrm{~Hz}, 1 \mathrm{H}), 6.56(\mathrm{t}, J=7.63 \mathrm{~Hz}, 1 \mathrm{H}), 6.20(\mathrm{~d}, J=$ $8.01 \mathrm{~Hz}, 1 \mathrm{H}), 3.49(\mathrm{~d}, J=14.11 \mathrm{~Hz}, 1 \mathrm{H}), 3.24(\mathrm{~d}, J=14.50 \mathrm{~Hz}, 1 \mathrm{H}), 3.13(\mathrm{tt}, J=11.59,3.10$ $\mathrm{Hz}, 1 \mathrm{H}), 2.82(\mathrm{~s}, 3 \mathrm{H}), 1.59-1.81(\mathrm{~m}, 5 \mathrm{H}), 1.43-1.53(\mathrm{~m}, 1 \mathrm{H}), 1.28-1.40(\mathrm{~m}, 1 \mathrm{H}), 1.12$ $1.26(\mathrm{~m}, 3 \mathrm{H}) .{ }^{13} \mathbf{C}$ NMR $\left(\mathbf{1 2 5} \mathbf{~ M H z}, \mathbf{C D C l}_{3}\right) \delta 207.8,147.5,135.5,131.1,127.8,126.7$, 125.8, 123.3, 121.0, 118.3, 105.9, 87.5, 45.3, 38.8, 31.5, 31.3, 29.5, 25.7, 25.6, 25.5. FTIR $\left(\mathbf{c m}^{-1}\right): 3023,2931,2857,1701,1581,1481,1358,1302,1118,1031,967$. HRMS(ESI): $\mathrm{m} / \mathrm{z}$ calculated for $\mathrm{C}_{22} \mathrm{H}_{26} \mathrm{ONS}[\mathrm{M}+\mathrm{H}]^{+}: 352.1730$, found: 352.1725 . 


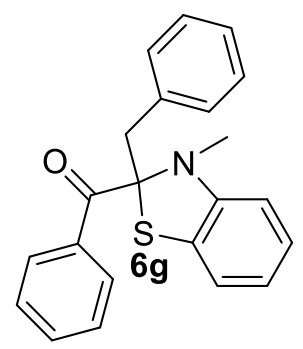

The titled compound was prepared by following the general procedure $\mathbf{F}$, obtained as a yellowish liquid (44 mg, 65\% yield). ${ }^{1} \mathbf{H}$ NMR (400 $\left.\mathbf{~ M H z}, \mathbf{C D C l}_{3}\right) \delta 7.99(\mathrm{~d}, J=7.33 \mathrm{~Hz}$, 2H), $7.51-7.45(\mathrm{~m}, 1 \mathrm{H}), 7.37-7.25(\mathrm{~m}, 4 \mathrm{H}), 7.19-7.11(\mathrm{~m}, 3 \mathrm{H}), 6.92(\mathrm{t}, J=8.24 \mathrm{~Hz}, 1 \mathrm{H})$, $6.85(\mathrm{~d}, J=7.33 \mathrm{~Hz}, 1 \mathrm{H}), 6.55(\mathrm{t}, J=7.33 \mathrm{~Hz}, 1 \mathrm{H}), 6.09(\mathrm{~d}, J=8.24 \mathrm{~Hz}, 1 \mathrm{H}), 3.53(\mathrm{~d}, J=$ $14.65 \mathrm{~Hz}, 1 \mathrm{H}), 3.47(\mathrm{~d}, J=14.65 \mathrm{~Hz}, 1 \mathrm{H}), 2.61(\mathrm{~s}, 3 \mathrm{H}),{ }^{13} \mathbf{C}$ NMR (100MHz, $\left.\mathbf{C D C l}_{3}\right) \delta$ $196.3,146.7,135.7,135.3,132.6,131.3,129.2$, 128.2, 127.6, 126.7, 126.0, 122.8, 121.1, 118.0, 105.7, 85.6, 42.5, 32.0. FTIR $\left(\mathbf{c m}^{-1}\right): 3030,2958,2922,2853,2822,1720,1676$, 1582, 1479, 1449, 1362, 1301, 1264, 1181, 1120, 1070, 1025, 908. HRMS(ESI): m/z calculated for $\mathrm{C}_{22} \mathrm{H}_{20} \mathrm{ONS}[\mathrm{M}+\mathrm{H}]^{+}: 346.1260$, found: 346.1257 .

(2-benzyl-3-methyl-2,3-dihydrobenzo[d]thiazol-2-yl)(4-methoxyphenyl)methanone (6h)

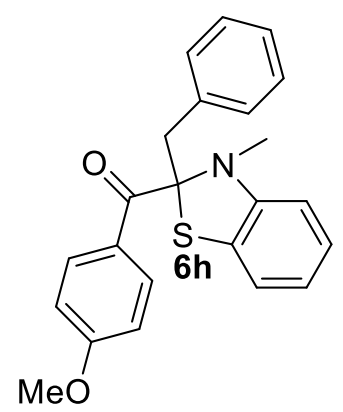

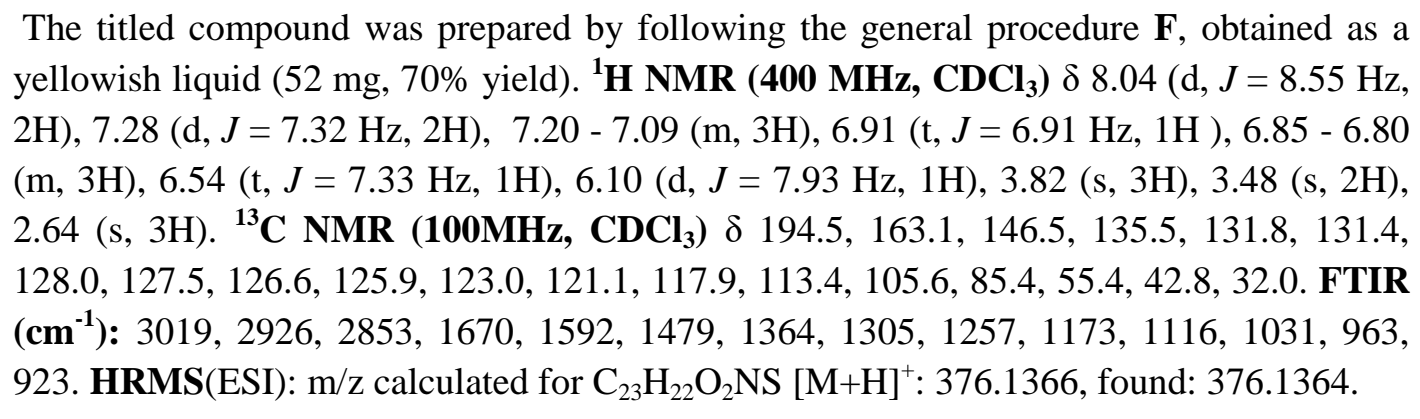

1-(2-(4-chlorobenzyl)-3-methyl-2,3-dihydrobenzo[d]thiazol-2-yl)ethan-1-one (6i)

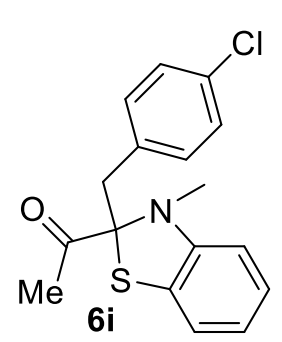

The titled compound was prepared by following the general procedure $\mathbf{F}$, obtained as a yellowish liquid (50 mg, 79\% yield). ${ }^{1} \mathbf{H}$ NMR (400 $\left.\mathbf{~ M H z , ~} \mathbf{C D C l}_{3}\right) \delta 7.22-7.15(\mathrm{~m}, 4 \mathrm{H})$, $6.93(\mathrm{t}, J=7.79 \mathrm{~Hz}, 1 \mathrm{H}), 6.85(\mathrm{~d}, J=8.70 \mathrm{~Hz}, 1 \mathrm{H}), 6.57(\mathrm{t}, J=8.01 \mathrm{~Hz}, 1 \mathrm{H}), 6.18(\mathrm{~d}, J=$ $7.79 \mathrm{~Hz}, 1 \mathrm{H}), 3.40(\mathrm{~d}, J=15.11 \mathrm{~Hz}, 1 \mathrm{H}), 3.23(\mathrm{~d}, J=14.65 \mathrm{~Hz}, 1 \mathrm{H}), 2.79(\mathrm{~s}, 3 \mathrm{H}), 2.33$ (s, 3H). ${ }^{13} \mathbf{C}$ NMR (100 MHz, $\left.\mathbf{C D C l}_{3}\right) \delta 202.8,147.5,134.2,132.7,132.4,128.0,126.1,123.1$, 121.2, 118.6, 106.0, 86.2, 38.0, 31.1, 25.2. FTIR $\left(\mathbf{c m}^{-1}\right): 3022,2956,2926,2869,2820,1707$, 1650, 1587, 1491, 1477, 1355, 1300, 1184, 1120, 1016. HRMS(ESI): $\mathrm{m} / \mathrm{z}$ calculated for $\mathrm{C}_{17} \mathrm{H}_{17} \mathrm{ONClS}[\mathrm{M}+\mathrm{H}]^{+}: 318.0714$, found: 318.1709.

\section{1-(2-(4-fluorobenzyl)-3-methyl-2,3-dihydrobenzo[d]thiazol-2-yl)ethan-1-one (6j)}

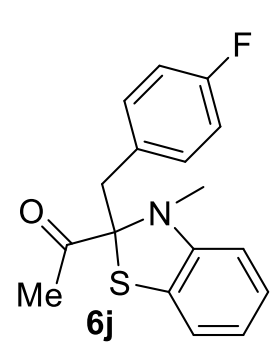

The titled compound was prepared by following the general procedure $\mathbf{F}$, obtained as a yellowish liquid (50 mg, 85\% yield. ${ }^{1} \mathbf{H}$ NMR (400 $\left.\mathbf{M H z}, \mathbf{C D C l}_{\mathbf{3}}\right) \delta 7.17-7.11(\mathrm{~m}, 2 \mathrm{H}), 6.88$ - $6.75(\mathrm{~m}, 4 \mathrm{H}), 6.49(\mathrm{t}, J=7.32 \mathrm{~Hz}, 1 \mathrm{H}), 6.11(\mathrm{~d}, J=7.33 \mathrm{~Hz}, 1 \mathrm{H}), 3.34(\mathrm{~d}, J=15.11 \mathrm{~Hz}$, $1 \mathrm{H}), 3.17(\mathrm{~d}, J=14.65 \mathrm{~Hz}, 1 \mathrm{H}), 2.73(\mathrm{~s}, 3 \mathrm{H}), 2.27(\mathrm{~s}, 3 \mathrm{H}) .{ }^{13} \mathbf{C}$ NMR (100 MHz, $\left.\mathbf{C D C l}_{3}\right) \delta$ 203.0, $163.0(\mathrm{C}-\mathrm{F}, 1 \mathrm{JC}-\mathrm{F}=244.4 \mathrm{~Hz}), 160.6(\mathrm{C}-\mathrm{F}, 1 \mathrm{JC}-\mathrm{F}=244.4 \mathrm{~Hz}), 147.5,132.6(\mathrm{C}-\mathrm{F}$, $3 \mathrm{JC}-\mathrm{F}=7.7 \mathrm{~Hz}), 132.5(\mathrm{C}-\mathrm{F}, 3 \mathrm{JC}-\mathrm{F}=7.7 \mathrm{~Hz}), 131.4(\mathrm{C}-\mathrm{F}, 4 \mathrm{JC}-\mathrm{F}=2.9 \mathrm{~Hz}), 131.3(\mathrm{C}-\mathrm{F}, 4 \mathrm{JC}-$ $\mathrm{F}=2.9 \mathrm{~Hz}), 126.1,123.2,121.2,118.5,114.8(\mathrm{C}-\mathrm{F}, 2 \mathrm{JC}-\mathrm{F}=21.1 \mathrm{~Hz}), 114.6(\mathrm{C}-\mathrm{F}, 2 \mathrm{JC}-\mathrm{F}=$ $21.1 \mathrm{~Hz}), 105.9,86.4,37.9,31.1,25.3 .{ }^{19} \mathbf{F} \mathbf{N M R}\left(\mathbf{C D C l}_{3}, 376 \mathbf{M H z}\right) \delta-116.15 \quad(\mathrm{~s}, 1 \mathrm{~F})$. FTIR $\left(\mathbf{c m}^{-1}\right):$ 2924, 2859, 1711, 1589, 1475, 1362, 1295, 1193, 1174, 1117, 1022, 909. HRMS(ESI): m/z calculated for $\mathrm{C}_{17} \mathrm{H}_{17}$ ONFS [M+H] $]^{+}: 302.1009$, found: 302.1004 .

\section{1-(2-(4-chlorobenzyl)-3-methyl-2,3-dihydrobenzo[d]thiazol-2-yl)-3-methylbutan-1-one (6k)}

The titled compound was prepared by following the general procedure $\mathbf{F}$, obtained as a yellowish liquid (60 $\mathrm{mg}$, $84 \%$ yield). ${ }^{1} \mathbf{H} \mathbf{~ N M R}\left(\mathbf{5 0 0} \mathbf{~ M H z}, \mathbf{C D C l}_{3}\right) \delta 7.22-7.14(\mathrm{~m}, 4 \mathrm{H}), 6.93(\mathrm{t}, J=8.39 \mathrm{~Hz}, 1 \mathrm{H}), 6.85(\mathrm{~d}, J=8.39 \mathrm{~Hz}$, 


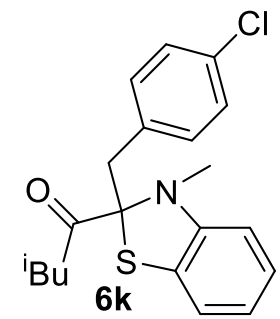

$1 \mathrm{H}), 6.58(\mathrm{t}, J=8.01 \mathrm{~Hz}, 1 \mathrm{H}), 6.17(\mathrm{~d}, J=8.01 \mathrm{~Hz}, 1 \mathrm{H}), 3.44(\mathrm{~d}, J=14.50 \mathrm{~Hz}, 1 \mathrm{H}), 3.22(\mathrm{~d}$, $J=14.88 \mathrm{~Hz}, 1 \mathrm{H}), 2.84(\mathrm{dd}, J=17.55,7.25 \mathrm{~Hz}, 1 \mathrm{H}), 2.78(\mathrm{~s}, 3 \mathrm{H}), 2.35(\mathrm{dd}, J=17.55,6.49$ $\mathrm{Hz}, 1 \mathrm{H}), 2.25-2.17(\mathrm{~m}, 1 \mathrm{H}), 0.89(\mathrm{~d}, J=6.87 \mathrm{~Hz}, 6 \mathrm{H}) .{ }^{13} \mathbf{C}$ NMR $\left(125 \mathbf{~ M H z}, \mathbf{C D C l}_{\mathbf{3}}\right) \delta$ 204.2, 147.4, 134.2, 132.7, 132.4, 128.0, 126.0, 123.1, 121.1, 118.5, 105.9, 86.5, 46.0, 38.4, 31.2, 24.3, 22.6, 22.4. FTIR $\left(\mathbf{c m}^{-1}\right):$ 2958, 2929, 2871, 2821, 1709, 1642, 1584, 1478, 1405, 1360, 1289, 1151, 1121, 1094, 1017, 911. HRMS(ESI): $\mathrm{m} / \mathrm{z}$ calculated for $\mathrm{C}_{20} \mathrm{H}_{23} \mathrm{ONClS}$ $[\mathrm{M}+\mathrm{H}]^{+}:$360.1183, found: 360.1183 .

\section{1-(2-(3-chlorobenzyl)-3-methyl-2,3-dihydrobenzo[d]thiazol-2-yl)-3-methylbutan-1-one (6l)}

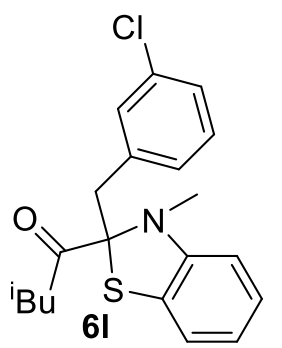

The titled compound was prepared by following the general procedure $\mathbf{F}$, obtained as a yellowish liquid (61 mg, 85\% yield). ${ }^{1} \mathbf{H}$ NMR (400 $\left.\mathbf{~ M H z , ~} \mathbf{C D C l}_{3}\right) \delta 7.35-7.33(\mathrm{~m}, 1 \mathrm{H})$, $7.29-7.26(\mathrm{~m}, 1 \mathrm{H}), 7.07-7.14(\mathrm{~m}, 2 \mathrm{H}), 6.94(\mathrm{t}, J=8.39 \mathrm{~Hz}, 1 \mathrm{H}), 6.83(\mathrm{~d}, J=9.16 \mathrm{~Hz}, 1 \mathrm{H})$, $6.56(\mathrm{t}, J=7.63 \mathrm{~Hz}, 1 \mathrm{H}), 6.23(\mathrm{~d}, J=7.63 \mathrm{~Hz}, 1 \mathrm{H}), 3.78(\mathrm{~d}, J=15.26 \mathrm{~Hz}, 1 \mathrm{H}), 3.38(\mathrm{~d}, J=$ $15.26 \mathrm{~Hz}, 1 \mathrm{H}$ ), 2.39 (dd, $J=17.55,6.87 \mathrm{~Hz}, 1 \mathrm{H}), 2.29-2.19(\mathrm{~m}, 1 \mathrm{H}), 0.94-0.89$ (m, 6H). ${ }^{13}$ C NMR (100 MHz, $\left.\mathbf{C D C l}_{3}\right) \delta 203.7,147.6,135.8,134.0,132.0,129.3,128.1,126.4$, 125.9, 123.4, 121.1, 118.4, 106.0, 86.7, 45.4, 34.2, 31.6, 24.4, 22.6, 22.4. FTIR (cm $\left.{ }^{-1}\right): 3019$, 2959, 2927, 2875, 1707, 1621, 1582, 1477, 1358, 1294, 1117, 1044, 910. HRMS(ESI): m/z calculated for $\mathrm{C}_{20} \mathrm{H}_{23} \mathrm{ONClS}[\mathrm{M}+\mathrm{H}]^{+}: 360.1183$, found: 360.1182 .

\section{1-(2-(2-chlorobenzyl)-3-methyl-2,3-dihydrobenzo[d]thiazol-2-yl)-3-methylbutan-1-one (6m)}

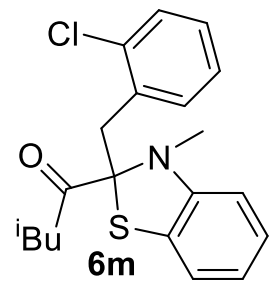

The titled compound was prepared by following the general procedure $\mathbf{F}$, obtained as a yellowish liquid (52 mg, 73\% yield). ${ }^{1} \mathbf{H}$ NMR (500 $\left.\mathbf{~ M H z}, \mathbf{C D C l}_{\mathbf{3}}\right) \delta 7.30-7.25(\mathrm{~m}, 1 \mathrm{H}), 7.17$ $-7.11(\mathrm{~m}, 3 \mathrm{H}), 6.96(\mathrm{t}, J=7.63 \mathrm{~Hz}, 1 \mathrm{H}), 6.89(\mathrm{~d}, J=7.25 \mathrm{~Hz}, 1 \mathrm{H}), 6.61(\mathrm{t}, J=7.25 \mathrm{~Hz}, 1 \mathrm{H})$, $6.21(\mathrm{~d}, J=7.63 \mathrm{~Hz}, 1 \mathrm{H}), 3.47(\mathrm{~d}, J=14.50 \mathrm{~Hz}, 1 \mathrm{H}), 3.26(\mathrm{~d}, J=14.50 \mathrm{~Hz}, 1 \mathrm{H}), 2.83(\mathrm{~s}, 3 \mathrm{H})$, $2.89(\mathrm{dd}, J=17.55,6.87 \mathrm{~Hz}, 1 \mathrm{H}), 2.39(\mathrm{dd}, J=17.74,6.29 \mathrm{~Hz}, 1 \mathrm{H}), 2.27-2.22(\mathrm{~m}, 1 \mathrm{H}), 2.39$ $(\mathrm{dd}, J=17.74,6.29 \mathrm{~Hz}, 1 \mathrm{H}), 0.92(\mathrm{~d}, J=6.10 \mathrm{~Hz}, 6 \mathrm{H}) .{ }^{13} \mathbf{C}$ NMR $\left(\mathbf{1 2 5} \mathbf{M H z}, \mathbf{C D C l}_{\mathbf{3}}\right) \delta 204.2$, $147.4,137.7,133.5,131.2,129.3,128.9,126.9,126.0,123.1,121.1,118.5,105.9,86.4,45.9$,

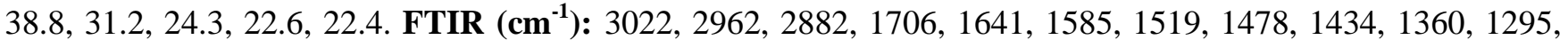
1161, 1114, 1031, 993, 926. HRMS(ESI): $\mathrm{m} / \mathrm{z}$ calculated for $\mathrm{C}_{20} \mathrm{H}_{23} \mathrm{ONClS}[\mathrm{M}+\mathrm{H}]^{+}: 360.1183$, found: 360.1181 .

\section{1-(2-(4-bromobenzyl)-3-methyl-2,3-dihydrobenzo[d]thiazol-2-yl)-3-methylbutan-1-one (6n)}

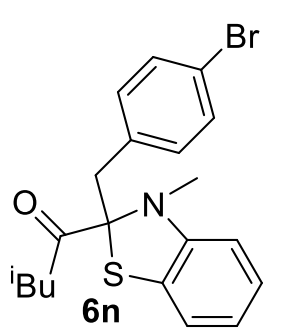

The titled compound was prepared by following the general procedure $\mathbf{F}$, obtained as a yellowish liquid (71 mg, 89\% yield). ${ }^{1} \mathbf{H}$ NMR $\left(\mathbf{4 0 0} \mathbf{~ M H z}, \mathbf{C D C l}_{\mathbf{3}}\right) \delta 7.32(\mathrm{~d}, J=8.39 \mathrm{~Hz}$, $2 \mathrm{H}), 7.13(\mathrm{~d}, J=8.39 \mathrm{~Hz}, 2 \mathrm{H}), 6.93(\mathrm{t}, J=8.39 \mathrm{~Hz}, 1 \mathrm{H}), 6.85(\mathrm{~d}, J=7.63 \mathrm{~Hz}, 1 \mathrm{H}), 6.58(\mathrm{t}, J$ $=7.63 \mathrm{~Hz}, 1 \mathrm{H}), 6.17(\mathrm{~d}, J=7.63 \mathrm{~Hz}, 1 \mathrm{H}), 3.43(\mathrm{~d}, J=14.50 \mathrm{~Hz}, 1 \mathrm{H}), 3.20(\mathrm{~d}, J=15.26 \mathrm{~Hz}$, $1 \mathrm{H}), 2.83(\mathrm{dd}, J=17.55,7.63 \mathrm{~Hz}, 1 \mathrm{H}), 2.78(\mathrm{~s}, 3 \mathrm{H}), 2.34(\mathrm{dd}, J=17.55,6.10 \mathrm{~Hz}, 1 \mathrm{H}), 2.26$ - $2.15(\mathrm{~m}, 1 \mathrm{H}), 0.88(\mathrm{~d}, J=6.10 \mathrm{~Hz}, 6 \mathrm{H}) .{ }^{\mathbf{1 3}} \mathbf{C}$ NMR (100 MHz, $\left.\mathbf{C D C l}_{\mathbf{3}}\right) \delta$ 204.1, 147.4, $134.7,132.8,130.9,126.0,123.0,121.1,120.8,118.5,105.8,86.3,45.9,38.4,31.2,24.3$, 22.6, 22.4. FTIR ( $\left.\mathbf{c m}^{-1}\right): 3020,2960,2880,2823,1707,1581,1480,1359,1295,1155,1116,1064,1019,982$, 924. HRMS(ESI): $\mathrm{m} / \mathrm{z}$ calculated for $\mathrm{C}_{20} \mathrm{H}_{23} \mathrm{ONBrS}[\mathrm{M}+\mathrm{H}]^{+}:$404.0678, found: 404.0676.

\section{1-(3-methyl-2-(4-methylbenzyl)-2,3-dihydrobenzo[d]thiazol-2-yl)ethan-1-one (6o)}

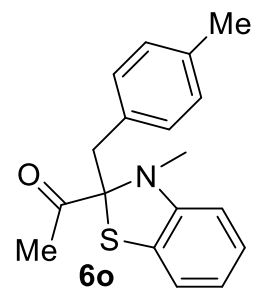

The titled compound was prepared by following the general procedure $\mathbf{F}$, obtained as a yellowish liquid (48 mg, 81\% yield). ${ }^{1} \mathbf{H}$ NMR (400 $\left.\mathbf{M H z}, \mathbf{C D C l}_{\mathbf{3}}\right) \delta 7.15(\mathrm{~d}, J=8.00 \mathrm{~Hz}$, 2H), $7.03(\mathrm{~d}, J=7.88 \mathrm{~Hz}, 2 \mathrm{H}), 6.92(\mathrm{t}, J=8.32 \mathrm{~Hz}, 1 \mathrm{H}), 6.85(\mathrm{~d}, J=8.50 \mathrm{~Hz}, 1 \mathrm{H}), 6.56(\mathrm{t}, J=$ $8.00 \mathrm{~Hz}, 1 \mathrm{H}), 6.19(\mathrm{~d}, J=7.75 \mathrm{~Hz}, 1 \mathrm{H}), 3.44(\mathrm{~d}, J=14.63 \mathrm{~Hz}, 1 \mathrm{H}), 3.25(\mathrm{~d}, J=14.63 \mathrm{~Hz}$, 
1H), 2.79 (s, 3H), 2.32 (s, 3H), 2.27 (s, 3H). ${ }^{13} \mathbf{C}$ NMR (100 MHz, $\left.\mathbf{C D C l}_{3}\right) \delta$ 203.0, 147.7, 136.3, 132.6, 130.9, 128.6, 126.0, 123.4, 121.2, 118.4, 105.9, 86.6, 38.2, 31.2, 25.3, 21.0. FTIR (cm $\left.{ }^{-1}\right):$ 3007, 2954, 2921, 2853, 2821, 1709, 1583, 1478, 1450, 1355, 1300, 1185, 1121, 1049, 1025, 912. HRMS(ESI): $\mathrm{m} / \mathrm{z}$ calculated for $\mathrm{C}_{18} \mathrm{H}_{20} \mathrm{ONS}$ $[\mathrm{M}+\mathrm{H}]^{+}: 298.1260$, found: 298.1258 .

\section{1-(3-methyl-2-(4-methylbenzyl)-2,3-dihydrobenzo[d]thiazol-2-yl)-2-phenylethan-1-one (6p)}

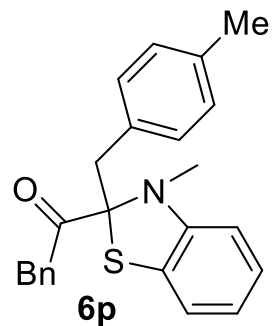

The titled compound was prepared by following the general procedure $\mathbf{F}$, obtained as a yellowish liquid (52 mg, 72\% yield). ${ }^{1} \mathbf{H}$ NMR (400 $\left.\mathbf{~ M H z , ~} \mathbf{C D C l}_{3}\right) \delta 7.33-7.19(\mathrm{~m}, 4 \mathrm{H})$, $7.18-7.10(\mathrm{~m}, 4 \mathrm{H}), 7.03(\mathrm{~d}, J=7.88 \mathrm{~Hz}, 2 \mathrm{H}), 6.99-6.89(\mathrm{~m}, 2 \mathrm{H}), 6.64-6.56(\mathrm{~m}, 1 \mathrm{H}), 6.21$ $(\mathrm{d}, J=7.88 \mathrm{~Hz}, 1 \mathrm{H}), 4.14(\mathrm{~d}, J=16.26 \mathrm{~Hz}, 1 \mathrm{H}), 3.89$ (d, $J=16.26 \mathrm{~Hz}, 1 \mathrm{H}), 3.49$ (d, $J=$ $14.63 \mathrm{~Hz}, 1 \mathrm{H}), 3.26(\mathrm{~d}, J=14.63 \mathrm{~Hz}, 1 \mathrm{H}), 2.73(\mathrm{~s}, 3 \mathrm{H}), 2.28(\mathrm{~s}, 3 \mathrm{H}) .{ }^{\mathbf{1 3}} \mathbf{C}$ NMR (100MHz, $\left.\mathbf{C D C l}_{3}\right) \delta 201.8,147.7,136.4,134.4,132.4,130.9,129.6,128.5,128.4,126.8,126.1,123.3$, 121.3, 118.5, 106.1, 87.0, 43.8, 38.5, 31.2, 21.0. FTIR $\left(\mathbf{c m}^{-1}\right): 3025,2923,2863,1714,1580$, 1481, 1358, 1301, 1120, 1030, 976. HRMS(ESI): $\mathrm{m} / \mathrm{z}$ calculated for $\mathrm{C}_{24} \mathrm{H}_{24} \mathrm{ONS}[\mathrm{M}+\mathrm{H}]^{+}: 374.1573$, found: 374.1570 .

\section{1-(2-(4-methoxybenzyl)-3-methyl-2,3-dihydrobenzo[d]thiazol-2-yl)ethan-1-one (6q) ${ }^{6}$}

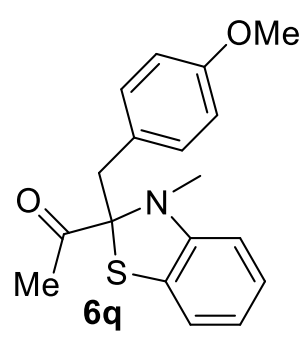

The titled compound was prepared by following the general procedure $\mathbf{F}$, obtained as a yellowish liquid (crude yield $55 \mathrm{mg}, 87 \%$ yield). ${ }^{1} \mathbf{H}$ NMR $\left(\mathbf{5 0 0} \mathbf{~ M H z}, \mathbf{C D C l}_{3}\right) \delta 7.18(\mathrm{~d}, J$ $=7.63 \mathrm{~Hz}, 2 \mathrm{H}), 6.92(\mathrm{t}, J=7.63 \mathrm{~Hz}, 1 \mathrm{H}), 6.85(\mathrm{~d}, J=7.25 \mathrm{~Hz}, 1 \mathrm{H}), 6.76(\mathrm{~d}, J=7.63 \mathrm{~Hz}$, $2 \mathrm{H}), 6.56(\mathrm{t}, J=7.25 \mathrm{~Hz}, 1 \mathrm{H}), 6.18(\mathrm{~d}, J=8.01 \mathrm{~Hz}, 1 \mathrm{H}), 3.74(\mathrm{~s}, 3 \mathrm{H}), 3.41(\mathrm{~d}, J=14.50$ $\mathrm{Hz}, 1 \mathrm{H}), 3.23(\mathrm{~d}, J=14.50 \mathrm{~Hz}, 1 \mathrm{H}), 2.79(\mathrm{~s}, 3 \mathrm{H}), 2.32$ (s, 3H). ${ }^{13} \mathbf{C}$ NMR (125 MHz, CDCl $\left._{3}\right) \delta 203.1,158.4,147.7,132.0,127.6,126.0,123.3,121.1,118.4,113.3,105.9,86.7$, 55.1, 37.9, 31.1, 25.4. FTIR $\left(\mathbf{c m}^{-1}\right): 3016,2925,2839,1707,1581,1512,1476,1357,1298$, 1247, 1183, 1119, 1034, 920. HRMS(ESI): m/z calculated for $\mathrm{C}_{18} \mathrm{H}_{20} \mathrm{O}_{2} \mathrm{NS}[\mathrm{M}+\mathrm{H}]^{+}: 314.1209$, found: 314.1204 .

\section{1-(3-methyl-2-(3-(trifluoromethyl)benzyl)-2,3-dihydrobenzo[d]thiazol-2-yl)ethan-1-one (6r)}

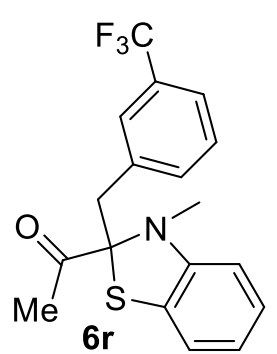

The titled compound was prepared by following the general procedure $\mathbf{F}$, obtained as a yellowish liquid (53 mg, 78\% yield). ${ }^{1} \mathbf{H}$ NMR (400 $\left.\mathbf{~ M H z , ~} \mathbf{C D C l}_{3}\right) \delta 7.50(\mathrm{~s}, 1 \mathrm{H}), 7.46(\mathrm{~d}, J=$ $8.39 \mathrm{~Hz}, 1 \mathrm{H}), 7.41(\mathrm{~d}, J=7.63 \mathrm{~Hz}, 1 \mathrm{H}), 7.30(\mathrm{t}, J=7.63 \mathrm{~Hz}, 1 \mathrm{H}), 6.91(\mathrm{t}, J=7.63 \mathrm{~Hz}, 1 \mathrm{H})$, $2.37(\mathrm{~s}, 3 \mathrm{H}), 6.82(\mathrm{~d}, J=7.63 \mathrm{~Hz}, 1 \mathrm{H}), 6.55(\mathrm{t}, J=7.63 \mathrm{~Hz}, 1 \mathrm{H}), 6.17(\mathrm{~d}, J=7.63 \mathrm{~Hz}, 1 \mathrm{H})$, $3.44(\mathrm{~d}, J=14.50 \mathrm{~Hz}, 1 \mathrm{H}), 3.32(\mathrm{~d}, J=14.50 \mathrm{~Hz}, 1 \mathrm{H}), 2.82(\mathrm{~s}, 3 \mathrm{H}), 2.37(\mathrm{~s}, 3 \mathrm{H}) .{ }^{13} \mathbf{C} \mathbf{~ N M R}$ $\left(\mathbf{1 0 0} \mathbf{~ M H z}, \mathbf{C D C l}_{3}\right) \delta 202.9,147.3,136.6,134.5,130.4(\mathrm{C}-\mathrm{F}, 2 \mathrm{JC}-\mathrm{F}=32.6 \mathrm{~Hz}), 130.1(\mathrm{C}-\mathrm{F}$, $2 \mathrm{JC}-\mathrm{F}=32.6 \mathrm{~Hz}), 129.8(\mathrm{C}-\mathrm{F}, 2 \mathrm{JC}-\mathrm{F}=32.6 \mathrm{~Hz}), 129.5(\mathrm{C}-\mathrm{F}, 2 \mathrm{JC}-\mathrm{F}=32.6 \mathrm{~Hz}), 128.2(\mathrm{C}-\mathrm{F}$, $1 \mathrm{JC}-\mathrm{F}=272.2 \mathrm{~Hz}), 128.1,127.9(\mathrm{C}-\mathrm{F}, 3 \mathrm{JC}-\mathrm{F}=3.8 \mathrm{~Hz}), 127.8(\mathrm{C}-\mathrm{F}, 3 \mathrm{JC}-\mathrm{F}=3.8 \mathrm{~Hz}), 127.8$ $(\mathrm{C}-\mathrm{F}, 3 \mathrm{JC}-\mathrm{F}=3.8 \mathrm{~Hz}), 127.8(\mathrm{C}-\mathrm{F}, 3 \mathrm{JC}-\mathrm{F}=3.8 \mathrm{~Hz}), 126.1,25.5(\mathrm{C}-\mathrm{F}, 1 \mathrm{JC}-\mathrm{F}=272.2 \mathrm{~Hz}), 123.7(\mathrm{C}-\mathrm{F}, 4 \mathrm{JC}-\mathrm{F}=$ $3.8 \mathrm{~Hz}), 123.6(\mathrm{C}-\mathrm{F}, 4 \mathrm{JC}-\mathrm{F}=3.8 \mathrm{~Hz}), 123.6(\mathrm{C}-\mathrm{F}, 4 \mathrm{JC}-\mathrm{F}=3.8 \mathrm{~Hz}), 123.5(\mathrm{C}-\mathrm{F}, 4 \mathrm{JC}-\mathrm{F}=3.8 \mathrm{~Hz}), 122.9,122.7$ $(\mathrm{C}-\mathrm{F}, 1 \mathrm{JC}-\mathrm{F}=272.2 \mathrm{~Hz}), 121.2,120.0(\mathrm{C}-\mathrm{F}, 1 \mathrm{JC}-\mathrm{F}=272.2 \mathrm{~Hz}), 118.6,106.0,86.1,38.6,31.2,25.1 .{ }^{19} \mathbf{F}$ NMR $\left(\mathbf{C D C l}_{3}, 376 \mathrm{MHz}\right) \delta$-62.59 (s, 3F). FTIR $\left(\mathbf{c m}^{-1}\right)$ : 3017, 2924, 2824, 1712, 1582, 1481, 1329, 1169, 1126, 1082, 1033, 925. HRMS(ESI): $\mathrm{m} / \mathrm{z}$ calculated for $\mathrm{C}_{18} \mathrm{H}_{17} \mathrm{ONF}_{3} \mathrm{~S}[\mathrm{M}+\mathrm{H}]^{+}: 352.0977$, found: 352.0973 .

\section{4-((2-acetyl-3-methyl-2,3-dihydrobenzo[d]thiazol-2-yl)methyl)benzonitrile (6s)}

The titled compound was prepared by following the general procedure $\mathbf{F}$, obtained as a yellowish liquid (50 $\mathrm{mg}$, 82\% yield). ${ }^{1} \mathbf{H}$ NMR (400 MHz, $\left.\mathbf{C D C l}_{\mathbf{3}}\right) \delta 7.49(\mathrm{~d}, J=8.39 \mathrm{~Hz}, 2 \mathrm{H}), 7.37(\mathrm{~d}, J=8.39 \mathrm{~Hz}, 2 \mathrm{H}), 6.94(\mathrm{t}, J=8.39$ $\mathrm{Hz}, 1 \mathrm{H}), 6.84(\mathrm{~d}, J=9.16 \mathrm{~Hz}, 1 \mathrm{H}), 6.58(\mathrm{t}, J=7.62 \mathrm{~Hz}, 1 \mathrm{H}), 6.19(\mathrm{~d}, J=7.63 \mathrm{~Hz}, 1 \mathrm{H}), 3.45(\mathrm{~d}, J=14.50 \mathrm{~Hz}$, 1H), $3.31(\mathrm{~d}, J=14.50 \mathrm{~Hz}, 1 \mathrm{H}), 2.82(\mathrm{~s}, 3 \mathrm{H}), 2.37$ (s, 3H). ${ }^{13} \mathbf{C}$ NMR (100 MHz, $\left.\mathbf{C D C l}_{\mathbf{3}}\right) \delta$ 202.7, 147.2, 141.5, 


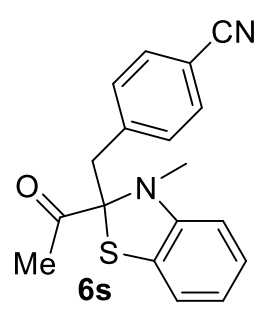

131.9, 131.4, 126.2, 122.8, 121.3, 118.9, 118.9, 110.6, 106.1, 85.9, 38.8, 31.1, 25.0. FTIR $\left(\mathbf{c m}^{-1}\right): 3020,2928,2231,1709,1580,1478,1356,1300,1122,1049,1025$. HRMS(ESI): $\mathrm{m} / \mathrm{z}$ calculated for $\mathrm{C}_{18} \mathrm{H}_{17} \mathrm{ON}_{2} \mathrm{~S}[\mathrm{M}+\mathrm{H}]^{+}: 309.1056$, found: 309.1052 .

1-(2-(furan-2-ylmethyl)-3-methyl-2,3-dihydrobenzo[d]thiazol-2-yl)-3-methylbutan-1-one (6t)

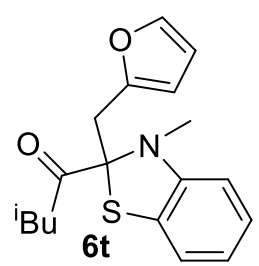

The titled compound was prepared by following the general procedure $\mathbf{F}$, obtained as a yellowish liquid (55 mg, 88\% yield). ${ }^{\mathbf{1}} \mathbf{H}$ NMR (400 $\left.\mathbf{~ M H z}, \mathbf{C D C l}_{3}\right) \delta 7.20$ (s, 1H), $6.94(\mathrm{t}, J=$ $7.78 \mathrm{~Hz}, 1 \mathrm{H}), 6.87$ (d, $J=7.33 \mathrm{~Hz}, 1 \mathrm{H}), 6.57$ (t, $J=7.33 \mathrm{~Hz}, 1 \mathrm{H}), 6.23-6.15$ (m, 2H), 6.13 (d, $J=3.21 \mathrm{~Hz}, 1 \mathrm{H}), 3.38(\mathrm{~s}, 2 \mathrm{H}), 2.95(\mathrm{dd}, J=17.86,7.33 \mathrm{~Hz}, 1 \mathrm{H}), 2.78(\mathrm{~s}, 3 \mathrm{H}), 2.38(\mathrm{dd}, J=$ 17.63, $6.18 \mathrm{~Hz}, 1 \mathrm{H}), 2.27-2.18(\mathrm{~m}, 1 \mathrm{H}), 0.91(\mathrm{~d}, J=6.41 \mathrm{~Hz}, 6 \mathrm{H}) .{ }^{13} \mathbf{C}$ NMR $(\mathbf{1 0 0} \mathbf{M H z}$, $\left.\mathbf{C D C l}_{3}\right) \delta 204.6,150.0,147.4,141.4,125.9,123.0,120.9,118.1,110.4,109.1,105.8,85.3$, 45.5, 32.9, 30.8, 24.2, 22.7, 22.3. FTIR ( $\left.\mathbf{c m}^{-1}\right):$ 3019, 2960, 2883, 2824, 1708, 1583, 1478, 1360, 1297, 1156, 1117, 1019, 931. HRMS(ESI): $\mathrm{m} / \mathrm{z}$ calculated for $\mathrm{C}_{18} \mathrm{H}_{22} \mathrm{O}_{2} \mathrm{NS}[\mathrm{M}+\mathrm{H}]^{+}: 316.1366$, found: 316.1363 .

\section{3-methyl-1-(3-methyl-2-(thiophen-2-ylmethyl)-2,3-dihydrobenzo[d]thiazol-2-yl)butan-1-one (6u)}

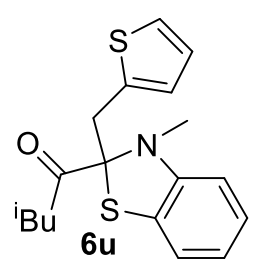

The titled compound was prepared by following the general procedure $\mathbf{F}$, obtained as a yellowish liquid (57 mg, 85\% yield). ${ }^{1} \mathbf{H}$ NMR (400 $\left.\mathbf{M H z}, \mathbf{C D C l}_{3}\right) \delta 7.12(\mathrm{dd}, J=5.04,1.37$ $\mathrm{Hz}, 1 \mathrm{H}), 6.95(\mathrm{t}, J=7.79 \mathrm{~Hz}, 1 \mathrm{H}), 6.91-6.87(\mathrm{~m}, 3 \mathrm{H}), 6.59(\mathrm{t}, J=7.32 \mathrm{~Hz}, 1 \mathrm{H}), 6.21(\mathrm{~d}, J=$ $7.33 \mathrm{~Hz}, 1 \mathrm{H}), 3.67(\mathrm{~d}, J=15.57 \mathrm{~Hz}, 1 \mathrm{H}), 3.51(\mathrm{~d}, J=16.41 \mathrm{~Hz}, 1 \mathrm{H}), 2.86(\mathrm{dd}, J=17.63,7.10$ $\mathrm{Hz}, 1 \mathrm{H}), 2.78(\mathrm{~s}, 3 \mathrm{H}), 2.33(\mathrm{dd}, J=17.63,7.10 \mathrm{~Hz}, 1 \mathrm{H}), 2.27-2.19(\mathrm{~m}, 1 \mathrm{H}), 0.90(\mathrm{~d}, J=6.41$ $\mathrm{Hz}, 6 \mathrm{H}) .{ }^{13} \mathbf{C}$ NMR (100 MHz, $\left.\mathbf{C D C l}_{\mathbf{3}}\right) \delta$ 204.1, 147.6, 137.4, 127.9, 126.0, 126.0, 125.5, 123.2, 121.2, 118.4, 105.9, 85.7, 45.7, 34.1, 30.7, 24.2, 22.6, 22.3. FTIR $\left(\mathbf{c m}^{-1}\right): 3018,2960,2882,2822,1706$, 1581, 1478, 1360, 1297, 1120, 1035, 918. HRMS(ESI): $\mathrm{m} / \mathrm{z}$ calculated for $\mathrm{C}_{18} \mathrm{H}_{22} \mathrm{ONS}_{2}[\mathrm{M}+\mathrm{H}]^{+}: 332.1137$, found: 332.1133 .

\section{1-(3-methyl-2-(1-phenylethyl)-2,3-dihydrobenzo[d]thiazol-2-yl)ethan-1-one (6v)}

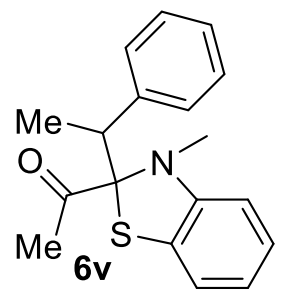

The titled compound was prepared by following the general procedure $\mathbf{F}$, found close spot in TLC and obtained mixture of yellowish liquid (47 mg, 79\% yield); $\mathrm{dr}=1.71: 1 .{ }^{\mathbf{1}} \mathbf{H}$ NMR (400 MHz, $\mathbf{C D C l}_{3}$ ) Major isomer: $\delta 7.38(\mathrm{~d}, J=7.63 \mathrm{~Hz}, 2 \mathrm{H}), 7.30-7.19(\mathrm{~m}, 3 \mathrm{H}), 7.02-$ $6.92(\mathrm{~m}, 2 \mathrm{H}), 6.64(\mathrm{t}, J=8.4 \mathrm{~Hz}, 1 \mathrm{H}), 6.27(\mathrm{~d}, J=7.63 \mathrm{~Hz}, 1 \mathrm{H}), 3.96(\mathrm{q}, J=6.87 \mathrm{~Hz}, 1 \mathrm{H})$, $2.82(\mathrm{~s}, 3 \mathrm{H}), 2.11(\mathrm{~s}, 3 \mathrm{H}), 1.44(\mathrm{~d}, J=6.87 \mathrm{~Hz}, 3 \mathrm{H})$; Minor Isomer: $7.46(\mathrm{~d}, J=6.86 \mathrm{~Hz}$, 2H), $7.17-7.10(\mathrm{~m}, 3 \mathrm{H}), 7.02-6.92(\mathrm{~m}, 1 \mathrm{H}), 6.81(\mathrm{t}, J=7.63 \mathrm{~Hz}, 1 \mathrm{H}), 6.54(\mathrm{t}, J=7.63 \mathrm{~Hz}$, $1 \mathrm{H}), 5.88(\mathrm{~d}, J=7.63 \mathrm{~Hz}, 1 \mathrm{H}), 3.83(\mathrm{q}, J=6.87 \mathrm{~Hz}, 1 \mathrm{H}), 2.50(\mathrm{~s}, 3 \mathrm{H}), 2.39(\mathrm{~s}, 3 \mathrm{H}), 1.34(\mathrm{~d}, J=6.87 \mathrm{~Hz}, 3 \mathrm{H})$. ${ }^{13}$ C NMR (100 MHz, $\left.\mathbf{C D C l}_{3}\right) \delta 203.5,202.4,147.7,147.5,142.1,140.4,129.6,129.1,128.0,127.8,127.1$, 126.6, 125.8, 123.6, 123.1, 121.0, 120.8, 118.5, 117.9, 105.9, 105.2, 91.2, 90.2, 41.5, 39.9, 30.7, 30.3, 28.4, 25.8,

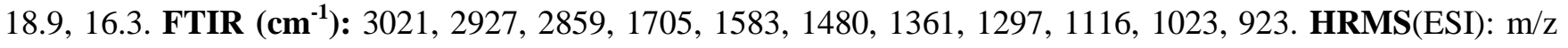
calculated for $\mathrm{C}_{18} \mathrm{H}_{20} \mathrm{ONS}[\mathrm{M}+\mathrm{H}]^{+}: 298.1260$, found: 298.1258 .

\section{1-(3-methyl-2-(1-(naphthalen-2-yl)ethyl)-2,3-dihydrobenzo[d]thiazol-2-yl)ethan-1-one (6w)}

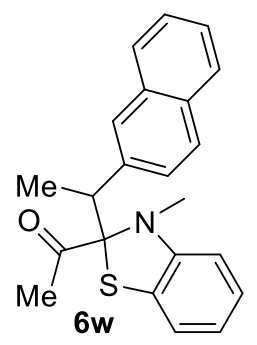

The titled compound was prepared by following the general procedure $\mathbf{F}$, obtained obtained as a yellowish liquid (47 mg, 67\% yield); dr = 2.34 : 1. H NMR (400 $\mathbf{~ M H z , ~} \mathbf{C D C l}_{3}$ ) $\mathbf{M a j o r}$ isomer: $\delta 7.94-7.70(\mathrm{~m}, 4 \mathrm{H}), 7.53(\mathrm{~d}, J=8.54 \mathrm{~Hz}, 1 \mathrm{H}), 7.50-7.39(\mathrm{~m}, 2 \mathrm{H}), 7.10-6.94(\mathrm{~m}$, $2 \mathrm{H}), 6.65(\mathrm{t}, J=7.93 \mathrm{~Hz}, 1 \mathrm{H}), 6.29(\mathrm{~d}, J=7.32 \mathrm{~Hz}, 1 \mathrm{H}), 4.14(\mathrm{q}, J=7.32 \mathrm{~Hz}, 1 \mathrm{H}), 2.85(\mathrm{~s}$, 
3H), $2.10(\mathrm{~s}, 3 \mathrm{H}), 1.54(\mathrm{~d}, J=7.32 \mathrm{~Hz}, 3 \mathrm{H})$, Minor isomer: $\delta 7.83-7.63(\mathrm{~m}, 5 \mathrm{H}), 7.44-7.35(\mathrm{~m}, 2 \mathrm{H}), 6.97(\mathrm{~d}, J$ $=7.32 \mathrm{~Hz}, 1 \mathrm{H}), 6.76(\mathrm{t}, J=7.93 \mathrm{~Hz}, 1 \mathrm{H}), 6.53(\mathrm{t}, J=7.32 \mathrm{~Hz}, 1 \mathrm{H}), 5.83(\mathrm{~d}, J=7.93 \mathrm{~Hz}, 1 \mathrm{H}), 4.03(\mathrm{q}, J=6.71$ $\mathrm{Hz}, 1 \mathrm{H}), 2.50(\mathrm{~s}, 3 \mathrm{H}), 2.40(\mathrm{~s}, 3 \mathrm{H}), 1.43(\mathrm{~d}, J=6.71 \mathrm{~Hz}, 3 \mathrm{H}) .{ }^{13} \mathbf{C} \mathbf{~ N M R}\left(\mathbf{1 0 0} \mathbf{~ M H z}, \mathbf{C D C l}_{3}\right)$ Major isomer: $\delta$ 203.3, 147.5, 138.1, 133.1, 132.5, 128.5, 128.0, 127.9, 127.5, 127.4, 125.9, 125.8, 123.7, 121.1, 118.6, 105.9, 91.4, 40.0, 30.2, 28.4, 16.5. FTIR (cm $\left.{ }^{-1}\right): 3020,2927,2862$, 1701, 1584, 1477, 1360, 1297, 1123, 1027, 919. HRMS(ESI): $\mathrm{m} / \mathrm{z}$ calculated for $\mathrm{C}_{22} \mathrm{H}_{22} \mathrm{ONS}[\mathrm{M}+\mathrm{H}]^{+}: 348.1417$, found: 348.1408 .

\section{1-(2-(cyclopropyl(phenyl)methyl)-3-methyl-2,3-dihydrobenzo[d]thiazol-2-yl)ethan-1-one (6x)}

The titled compound was prepared by following the general procedure $\mathbf{F}$, found close spot in TLC and obtained as a mixtue of yellowish liquid (48 $\mathrm{mg}, 75 \%$ yield); $\left.\mathrm{dr}=2: 1 .{ }^{1} \mathbf{H} \mathbf{~ N M R ~ ( 5 0 0 ~} \mathbf{M H z}, \mathbf{C D C l}_{\mathbf{3}}\right) \mathrm{dr}=1.96: 1$. Major isomer : $\delta 7.44-7.35(\mathrm{~m}, 2 \mathrm{H}), 7.34-7.16(\mathrm{~m}, 3 \mathrm{H}), 7.05-6.97(\mathrm{~m}, 2 \mathrm{H}), 6.66(\mathrm{t}, J=7.25 \mathrm{~Hz}, 1 \mathrm{H}), 6.30(\mathrm{~d}, J=$ $8.01 \mathrm{~Hz}, 1 \mathrm{H}), 3.17(\mathrm{~d}, J=9.92 \mathrm{~Hz}, 1 \mathrm{H}), 2.85(\mathrm{~s}, 3 \mathrm{H}), 2.20(\mathrm{~s}, 3 \mathrm{H}), 1.72-1.62(\mathrm{~m}, 1 \mathrm{H}), 0.69-0.62(\mathrm{~m}, 1 \mathrm{H}), 0.56$

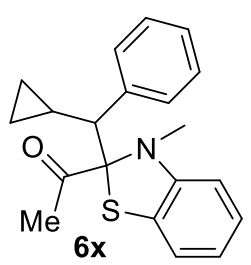
- $0.39(\mathrm{~m}, 3 \mathrm{H})$; Minor Isomer: $7.52(\mathrm{~d}, J=6.87 \mathrm{~Hz}, 2 \mathrm{H}), 7.44-7.35(\mathrm{~m}, 1 \mathrm{H}), 7.34-7.16(\mathrm{~m}$, 2H), 7.05- $6.97(\mathrm{~m}, 1 \mathrm{H}), 6.87(\mathrm{t}, J=7.82 \mathrm{~Hz}, 1 \mathrm{H}), 6.61(\mathrm{t}, J=7.82 \mathrm{~Hz}, 1 \mathrm{H}), 5.95(\mathrm{~d}, J=8.01$ $\mathrm{Hz}, 1 \mathrm{H}), 3.29(\mathrm{~d}, J=8.77 \mathrm{~Hz}, 1 \mathrm{H}), 2.48(\mathrm{~s}, 3 \mathrm{H}), 2.38(\mathrm{~s}, 3 \mathrm{H}), 1.23-1.15(\mathrm{~m}, 1 \mathrm{H}), 0.69-0.62$ $(\mathrm{m}, 1 \mathrm{H}), 0.56-0.39(\mathrm{~m}, 1 \mathrm{H}), 0.38-0.33(\mathrm{~m}, 1 \mathrm{H}), 0.23-0.18(\mathrm{~m}, 1 \mathrm{H}) .{ }^{13} \mathbf{C} \mathbf{~ N M R}\left(\mathbf{C D C l}_{3}, \mathbf{1 2 5}\right.$ MHz) $\delta 202.8,202.6,147.9,147.6,140.5,140.3,130.4,129.7,128.4,127.7,127.6$, $126.8,126.6,125.9,123.7,123.5,120.9,120.7,118.3,118.1,105.8,105.5,91.3,90.1,51.2$, 50.1, 31.7, 30.7, 28.1, 27.0, 14.8, 12.7, 8.3, 6.2, 4.6, 3.2. FTIR $\left(\mathbf{c m}^{-1}\right): 3003,2956,2923,2853,2821,1703,1680$, $1581,1478,1451,1353,1302,1123,1025,917 . \mathbf{H R M S}(\mathrm{ESI}): \mathrm{m} / \mathrm{z}$ calculated for $\mathrm{C}_{20} \mathrm{H}_{22} \mathrm{ONS}[\mathrm{M}+\mathrm{H}]^{+}: 324.1417$, found: 324.1408 .

\section{1-(2-benzhydryl-3-methyl-2,3-dihydrobenzo[d]thiazol-2-yl)-3-methylbutan-1-one (6y)}

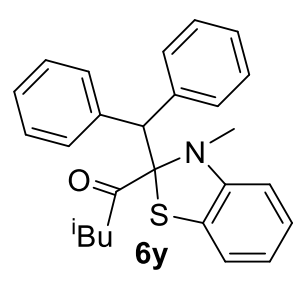

The titled compound was prepared by following the general procedure $\mathbf{F}$, obtained obtained as a yellowish liquid (57 mg, 71\% yield). ${ }^{1} \mathbf{H}$ NMR (500 $\left.\mathbf{~ M H z , ~} \mathbf{C D C l}_{\mathbf{3}}\right) \delta 7.69(\mathrm{~d}, J=7.63$ $\mathrm{Hz}, 2 \mathrm{H}), 7.40$ (t, $J=7.25 \mathrm{~Hz}, 1 \mathrm{H}), 7.37-7.20(\mathrm{~m}, 7 \mathrm{H}), 7.06$ (d, $J=7.25 \mathrm{~Hz}, 1 \mathrm{H}), 6.99$ (t, $J$ $=7.63 \mathrm{~Hz}, 1 \mathrm{H}), 6.70(\mathrm{t}, J=7.25 \mathrm{~Hz}, 1 \mathrm{H}), 6.10(\mathrm{~d}, J=7.63 \mathrm{~Hz}, 1 \mathrm{H}), 5.57(\mathrm{~s}, 1 \mathrm{H}), 2.89(\mathrm{dd}, J$ $=17.74,5.53 \mathrm{~Hz}, 1 \mathrm{H}), 2.55-2.44(\mathrm{~m}, 4 \mathrm{H}), 2.29-2.15(\mathrm{~m}, 1 \mathrm{H}), 0.90(\mathrm{~d}, J=6.10 \mathrm{~Hz}, 3 \mathrm{H})$, $0.84(\mathrm{~d}, J=6.10 \mathrm{~Hz}, 3 \mathrm{H}) .{ }^{13} \mathbf{C} \mathbf{N M R}\left(\mathbf{C D C l}_{3}, \mathbf{1 2 5} \mathbf{M H z}\right) \delta 202.5,147.3,139.8,130.1$, 130.1, 129.0, 128.6, 128.1, 128.0, 127.1, 126.8, 126.6, 126.0, 123.6, 121.0, 118.3, 105.7, 89.5, 50.7, 47.4, 30.2, 24.6, 22.5, 22.3. FTIR $\left(\mathrm{cm}^{-1}\right):$ 2924, 2859, 1722, 1675, 1591, 1463, 1316, 1276, 1178, 1120, 1068, 1031, 933. HRMS(ESI): $\mathrm{m} / \mathrm{z}$ calculated for $\mathrm{C}_{26} \mathrm{H}_{28} \mathrm{ONS}[\mathrm{M}+\mathrm{H}]^{+}:$402.1886, found: 402.1882 .

\section{Characterization of $\mathrm{d}[1,3]$ derived products:}

\section{1-phenylbutane-2,3-dione and 3-hydroxy-4-phenylbut-3-en-2-one $(8 a)^{8}$}

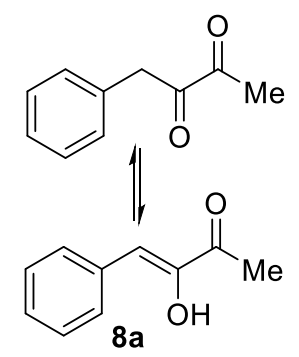

The titled compound was prepared by following the general procedure $\mathbf{H}(\mathbf{a})$, obtained as a liquid (20 mg, 82\% yield). ${ }^{1} \mathbf{H}$ NMR (400 $\mathbf{~ M H z , ~} \mathbf{C D C l}_{\mathbf{3}}$ ) Keto form $\delta 7.34-7.30(\mathrm{~m}, 1 \mathrm{H})$, 7.29 - 7.27 (m, 4H), 4.04 (s, 2H), 2.32 (s, 3H). Enol form: 7.83 (d, J=8.84 Hz, 2H), 7.44 $7.36(\mathrm{~m}, 2 \mathrm{H}), 7.34-7.30(\mathrm{~m}, 1 \mathrm{H}), 6.45(\mathrm{~s}, 1 \mathrm{H}), 2.51(\mathrm{~s}, 3 \mathrm{H})$.

\section{1-(4-methoxyphenyl)-3-phenylpropane-1,2-dione (8b) ${ }^{9}$}

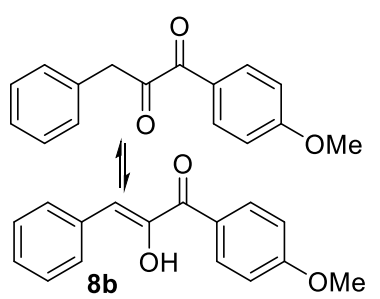

The titled compound was prepared by following the general procedure $\mathbf{H}(\mathbf{a})$, obtained as a yellowish liquid (18 mg, 68\% yield). ${ }^{1} \mathbf{H}$ NMR (400 $\mathbf{M H z}, \mathbf{C D C l}_{3}$ ) Keto form $\delta 7.89$ 
$(\mathrm{d}, 9.16 \mathrm{~Hz}, 2 \mathrm{H}), 7.42-7.38(\mathrm{~m}, 1 \mathrm{H}), 7.35-7.29(\mathrm{~m}, 2 \mathrm{H}), 7.28-7.23(\mathrm{~m}, 2 \mathrm{H}), 6.90(\mathrm{~d}, J=9.16 \mathrm{~Hz}, 2 \mathrm{H}), 4.18(\mathrm{~s}$, 2H), $3.86(\mathrm{~s}, 3 \mathrm{H})$. Enol form: $7.83(\mathrm{~d}, J=8.47 \mathrm{~Hz}, 2 \mathrm{H}), 7.78(\mathrm{~d}, J=8.70 \mathrm{~Hz}, 2 \mathrm{H}), 7.42-7.38(\mathrm{~m}, 1 \mathrm{H}), 7.35$ $7.29(\mathrm{~m}, 1 \mathrm{H}), 7.28-7.23(\mathrm{~m}, 2 \mathrm{H}), 7.00(\mathrm{~d}, J=9.16 \mathrm{~Hz}, 1 \mathrm{H}), 6.40(\mathrm{~s}, 1 \mathrm{H}), 3.90(\mathrm{~s}, 3 \mathrm{H})$.

\section{(Z)-1-(furan-2-yl)-2-hydroxy-5-methylhex-1-en-3-one (8c)}

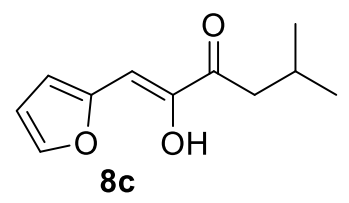

The titled compound was prepared by following the general procedure $\mathbf{H}(\mathbf{a})$, obtained as a liquid (13 mg, 63\% yield). ${ }^{\mathbf{1}} \mathbf{H}$ NMR (400 $\left.\mathbf{~ M H z}, \mathbf{C D C l}_{3}\right) \delta 7.50(\mathrm{dd}, J=1.81,0.69 \mathrm{~Hz}$, $1 \mathrm{H}), 7.25(\mathrm{~d}, J=1.63 \mathrm{~Hz}, 1 \mathrm{H}), 7.02(\mathrm{~d}, J=3.38 \mathrm{~Hz}, 1 \mathrm{H}), 6.56-6.51(\mathrm{~m}, 2 \mathrm{H}), 2.62(\mathrm{~d}, J=$ $7.00 \mathrm{~Hz}, 2 \mathrm{H}), 2.23(\mathrm{~m}, 1 \mathrm{H}), 0.98(\mathrm{~d}, J=6.63 \mathrm{~Hz}, 6 \mathrm{H}) .{ }^{13} \mathbf{C ~ N M R}\left(\mathbf{1 0 0} \mathbf{M H z}, \mathbf{C D C l}_{3}\right) \delta$ 196.3, 149.9, 145.3, 143.2, 114.4, 112.7, 102.7, 43.7, 26.3, 22.7. FTIR $\left(\mathbf{c m}^{-1}\right): 2962,2931,2872,1714,1664$, 1629, 1472, 1380, 1331, 1166, 1091, 1021. HRMS(ESI): $\mathrm{m} / \mathrm{z}$ calculated for $\mathrm{C}_{11} \mathrm{H}_{15} \mathrm{O}_{3}[\mathrm{M}+\mathrm{H}]^{+}:$195.1016, found: 195.1014.

\section{3-hydroxy-1-phenylbutan-2-one (9) ${ }^{11}$}<smiles>CC(O)C(=O)Cc1ccccc1</smiles>

The titled compound was prepared by following the general procedure $\mathbf{H}(\mathbf{b})$, obtained as a liquid (13mg, 58\% yield). ${ }^{\mathbf{1}} \mathbf{H}$ NMR (400 $\left.\mathbf{~ M H z}, \mathbf{C D C l}_{\mathbf{3}}\right) \delta 7.37-7.31(\mathrm{~m}, 2 \mathrm{H}), 7.31-7.26$ $(\mathrm{m}, 1 \mathrm{H}), 7.23-7.18(\mathrm{~m}, 2 \mathrm{H}), 4.35(\mathrm{q}, J=7.09 \mathrm{~Hz}, 1 \mathrm{H}), 3.87-3.74(\mathrm{~m}, 2 \mathrm{H}), 1.42(\mathrm{~d}, J=$ $7.13 \mathrm{~Hz}, 3 \mathrm{H})$.

\section{2-benzyl-3-methyl-2-(prop-1-en-2-yl)-2,3-dihydrobenzo[d] thiazole (S14)}<smiles>C=C(C)C1(Cc2ccccc2)Sc2ccccc2N1C</smiles>

HRMS(ESI): $\mathrm{m} / \mathrm{z}$ calculated for $\mathrm{C}_{18} \mathrm{H}_{20} \mathrm{NS}[\mathrm{M}+\mathrm{H}]^{+}: 282.1323$, found: 282.1316 .

The titled compound was prepared by following the general procedure $\mathbf{H}(\mathbf{c})$, obtained as a liquid (61 mg, 86\% yield). ${ }^{1} \mathbf{H}$ NMR (500 MHz, $\left.\mathbf{C D C l}_{3}\right) \delta 7.29(\mathrm{~d}, J=7.25 \mathrm{~Hz}, 2 \mathrm{H}), 7.19$ $7.09(\mathrm{~m}, 3 \mathrm{H}), 6.80(\mathrm{t}, J=8.20 \mathrm{~Hz}, 1 \mathrm{H}), 6.75(\mathrm{~d}, J=7.63 \mathrm{~Hz}, 1 \mathrm{H}), 6.41(\mathrm{t}, J=7.44 \mathrm{~Hz}, 1 \mathrm{H})$, $6.01(\mathrm{~d}, J=7.63 \mathrm{~Hz}, 1 \mathrm{H}), 5.18(\mathrm{~s}, 1 \mathrm{H}), 5.12(\mathrm{~s}, 1 \mathrm{H}), 3.43(\mathrm{~d}, J=14.50 \mathrm{~Hz}, 1 \mathrm{H}), 3.25(\mathrm{~d}, J=$ $14.11 \mathrm{~Hz}, 1 \mathrm{H}), 2.81$ (s, 3H), 1.98 (s, 3H). ${ }^{13} \mathbf{C}$ NMR (125 MHz, $\left.\mathbf{C D C l}_{3}\right) \delta$ 147.8, 146.7, 136.4, 131.1, 127.3, 126.4, 124.9, 124.2, 120.3, 116.8, 113.4, 104.2, 84.5, 42.7, 29.9, 20.0. FTIR $\left(\mathbf{c m}^{-1}\right)$ : 2921, 2868, 2818, 1638, 1581, 1483, 1365, 1301,1221, 1118, 1023, 907. 3-methyl-1-phenylbut-3-en-2-one (10) $)^{13}$<smiles>C=C(C)C(=O)Cc1ccccc1</smiles>

The titled compound was prepared by following the general procedure $\mathbf{H}(\mathbf{a})$, obtained as a liquid (11 mg, 69\% yield). Ketone: Enol = 1: $0.11 .{ }^{1} \mathbf{H}$ NMR (200 $\left.\mathbf{~ M H z , ~} \mathbf{C D C l}_{3}\right) \delta$ $7.18-7.32(\mathrm{~m}, 5 \mathrm{H}), 6.08(\mathrm{~m}, 1 \mathrm{H}), 5.84(\mathrm{~m}, 1 \mathrm{H}), 4.01(\mathrm{~s}, 2 \mathrm{H}), 1.8(\mathrm{~m}, 3 \mathrm{H})$. The product show $\sim 11 \%$ enol form.

\section{References}

(1) Claudi, F.; Siniscalchi, A.; Marucci, G.; Stefano, A. D.; Accornni, B.; Giorgioni, G. Benzimidazole, Benzoxazole and Benzothiazole Derivatives AS $5 \mathrm{HT}_{2 \mathrm{~B}}$ Receptor Ligands. Synthesis and Preliminary Pharmacological Evaluation. Med. Chem. Res. 2005, 14, 57-73.

(2) Alam. M. N.;Lakshmi, M. K.; Maity, P. A Removable Functional Group Strategy for Regiodivergent Wittig Rearrangement Products. Org. Biomol. Chem 2018, 16, 8922-8926.

(3) Senaweera, S.; Weaver, J. D.; S $\mathrm{N}_{\mathrm{N}}$ Ar Catalysis Enhanced by an Aromatic Donor - Acceptor Interaction; Facile Access to Chlorinated Polyfluoroarenes. Chem. Commun. 2017, 53, 7545-7548.

(4) Nicolaou, K. C.; Rhoades, D.; Wang, Y.; Bai, R.; Hamel, E.; Aujay, M.; Sandoval, J.; Guvrilyuk, J. 12, 13Aziridinyl Epothilones. Stereoselective Synthesis of Trisubstituted Olefinic Bonds from Methyl Ketones and 
Heteroaromatic Phosphonates and Design, Synthesis, and Biological Evaluation of Potent Antitumor Agents. $J$. Am . Chem. Soc. 2017, 139, 7318-7334.

(5) Itoh, K.; Ori, K.; Ishibaba, M.; Chikashita, H. General Reactivity of 2-Lithiobenzothiazole to Variou Electrophiles and the Use as a formyl Anion Equivallent in the Synthesis of $\alpha$-Hydroxy Carbonyl Compounds. Bull. Chem. Soc. Jpn. 1988, 61, 3637-3648.

(6) Silica sensitive compound, ${ }^{1} \mathrm{H}$ NMR and ${ }^{13} \mathrm{C}$ was taken as crude

(7) (a) Itoh, K.; Inoue, K.; Ishimoto, N.; Komazawa, S-I.; Chikashita, H. Nonacidic and Highly Chemoselective Protection of the Carbonyl Function. 3-Methylbenzothiazolines as a Base- and Acid-Resistant Protected Form for the Carbonyl groups. Bull. Chem. Soc. Jpn. 1989, 62, 1215-1225; (b) Boger, L. D.; Corey, E. J. Benzothiazole as Carbonyl Equivalents. Tetrahedron Lett. 1978, 1, 5-8; (c) Richheimer, L. S.; Altman, L. J. An Aldehyde Synthesis Utilizing the Thiazole Ring System. Tetrahedron Lett. 1971, 49, 4709-4711. (d) Gaul, C.; Seebach, D. A Valine-Derived Lithiated 3-Methylthiomethyl-1,3-oxazolidine-2-one for Enantioselective Nucleophilic Hydroxymethylation, Formylationand Alkoxycarbonylation of Aldehydes. Org. Lett. 2000, 2, 1501-1504

(8) Chen, C-T.; Kao, J-Q.; Salunke, S. B.; Lin, Y-H. Enantioselective Aerobic Oxidation of r-Hydroxy-Ketones Catalyzed by Oxidovanadium(V) Methoxides Bearing Chiral, N-Salicylidene-tert-butylglycinates. Org. Lett. 2011, 13, 26-29

(9) He, Z.; Qi, X.; She, Z.; Zhao, Y.; Li, S.; Tang, J.; Gao, G.; Lan, Y.; You, J. Room-Temperature Coupling/Decarboxylation Reaction of $\alpha$-Oxocarboxylates with $\alpha$-Bromoketones: Solvent-Controlled Regioselectivity for 1, 2- and 1,3-Diketones. J. Org. Chem. 2017, 82, 1403-1411.

(10) Pedrini, P.; Medici, A.; Fogagnolo, M.; Fantin, G.; Dondoni, A. Thiazolylmethylenetriphenylphosporane and its Benzo derivative: Stable and Practical Wittig Reagents for the Synthesis of vinylthiazoles. Two Carbon Homologation of Aldehydes. Tetrahedron Lett. 1988, 44, 2021-2031.

(11) Ley, V. S.; Mitchll, C.; Pears, D.; Ramarao, C.; Yu, J-Q.; Zhou, W. Recyclable Polyurea-Microencapsulated Pd (0) Nanoparticles: An Efficient Catalyst for Hydrogenolysis of Epoxides. Org. Lett. 2003, 5, 4665-4668.

(12) Miele, M.; Citarella, A.; Micale, N.; Holzer, W.; Pace, V. Direct and Chemoselective Synthesis of tertiary difluoroketones via Weinreb Amide Homologation with a $\mathrm{CHF}_{2}-$ Carbene Equivalent. Org. lett. 2019, 21 , $8261-8265$.

(13) (a) Satoh, T.; Itoh, M.; Ohara, T.; Yamakawa, K. $\alpha, \beta$ - Epoxy Sulfoxides as Useful Intermediates in Organic Synthesis. An Improved Sythesis of $\alpha, \beta$ - Unsaturated Carbonyl Compounds from Carbonyl Compounds with Carbon Homologation through $\alpha$, $\beta$ - Epoxy Sulfoxides. Bull. Chem. Soc. Jpn. 1987, 60, 1839-1846. (b) Imamoto, T.; Takiyama, N.; Nakamura, K.; Hatajima, T.; Kamiya, Y. Reactions of Carbonyl Compounds with Grignard Reagents in the Presence of Cerium Chloride. J. Am . Chem. Soc. 1989, 111, 4392-4398.

(14) Gaussian 09, Revision A.01, M. J. Frisch, G. W. Trucks, H. B. Schlegel, G. E. Scuseria, M. A. Robb, J. R. Cheeseman, G. Scalmani, V. Barone, B. Mennucci, G. A. Petersson, H. Nakatsuji, M. Caricato, X. Li, H. P. Hratchian, A. F. Izmaylov, J. Bloino, G. Zheng, J. L. Sonnenberg, M. Hada, M. Ehara, K. Toyota, R. Fukuda, J. Hasegawa, M. Ishida, T. Nakajima, Y. Honda, O. Kitao, H. Nakai, T. Vreven, J. A. Montgomery, Jr., J. E. Peralta, F. Ogliaro, M. Bearpark, J. J. Heyd, E. Brothers, K. N. Kudin, V. N. Staroverov, R. Kobayashi, J. Normand, K. Raghavachari, A. Rendell, J. C. Burant, S. S. Iyengar, J. Tomasi, M. Cossi, N. Rega, J. M. Millam, M. Klene, J. E. Knox, J. B. Cross, V. Bakken, C. Adamo, J. Jaramillo, R. Gomperts, R. E. Stratmann, O. Yazyev, A. J. Austin, R. Cammi, C. Pomelli, J. W. Ochterski, R. L. Martin, K. Morokuma, V. G. Zakrzewski, G. A. Voth, P. Salvador, J. J. Dannenberg, S. Dapprich, A. D. Daniels, O. Farkas, J. B. Foresman, J. V. Ortiz, J. Cioslowski, and D. J. Fox, Gaussian, Inc., Wallingford CT, 2009.

(15) Chai, J.-D.; Head-Gordon, M. Long-range Corrected hybrid density functionals with damped atom-atom dispersion corrections. Phys. Chem. Chem. Phys. 2008, 10, 6615.

(16) Becke, A. D. Density-functional thermochemistry. III. The role of exact exchange. J. Chem. Phys. 1993, 98, $5648-5652$.

(17) Marenich, A. V.; Cramer, C. J.; Truhlar, D. G. Universal Solvation Model Based on Solute Electron Density and on a Continuum Model of the Solvent Defined by the Bulk Dielectric Constant and Atomic Surface Tensions. J. Phys. Chem. B. 2009, 113, 6378-6396. 


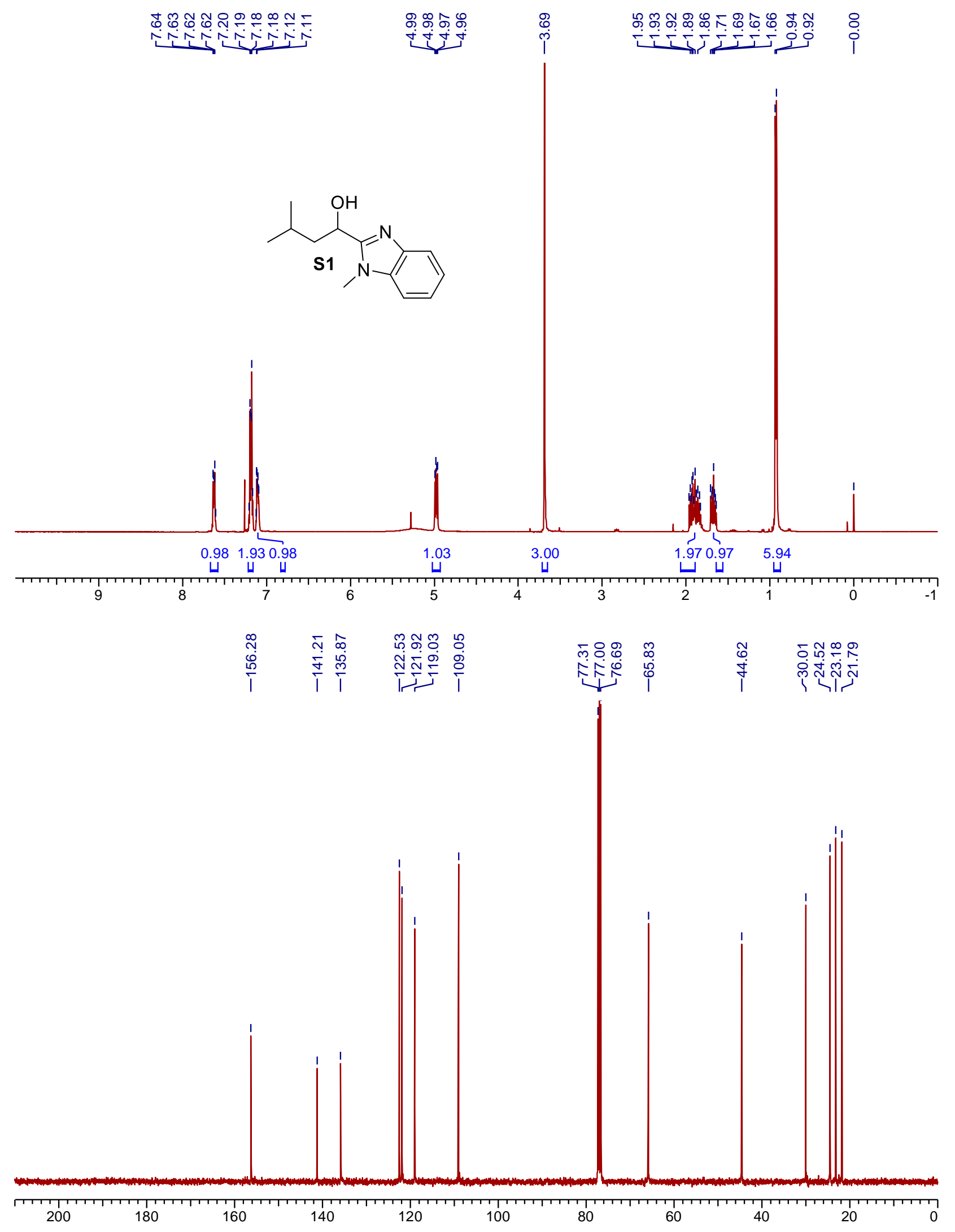




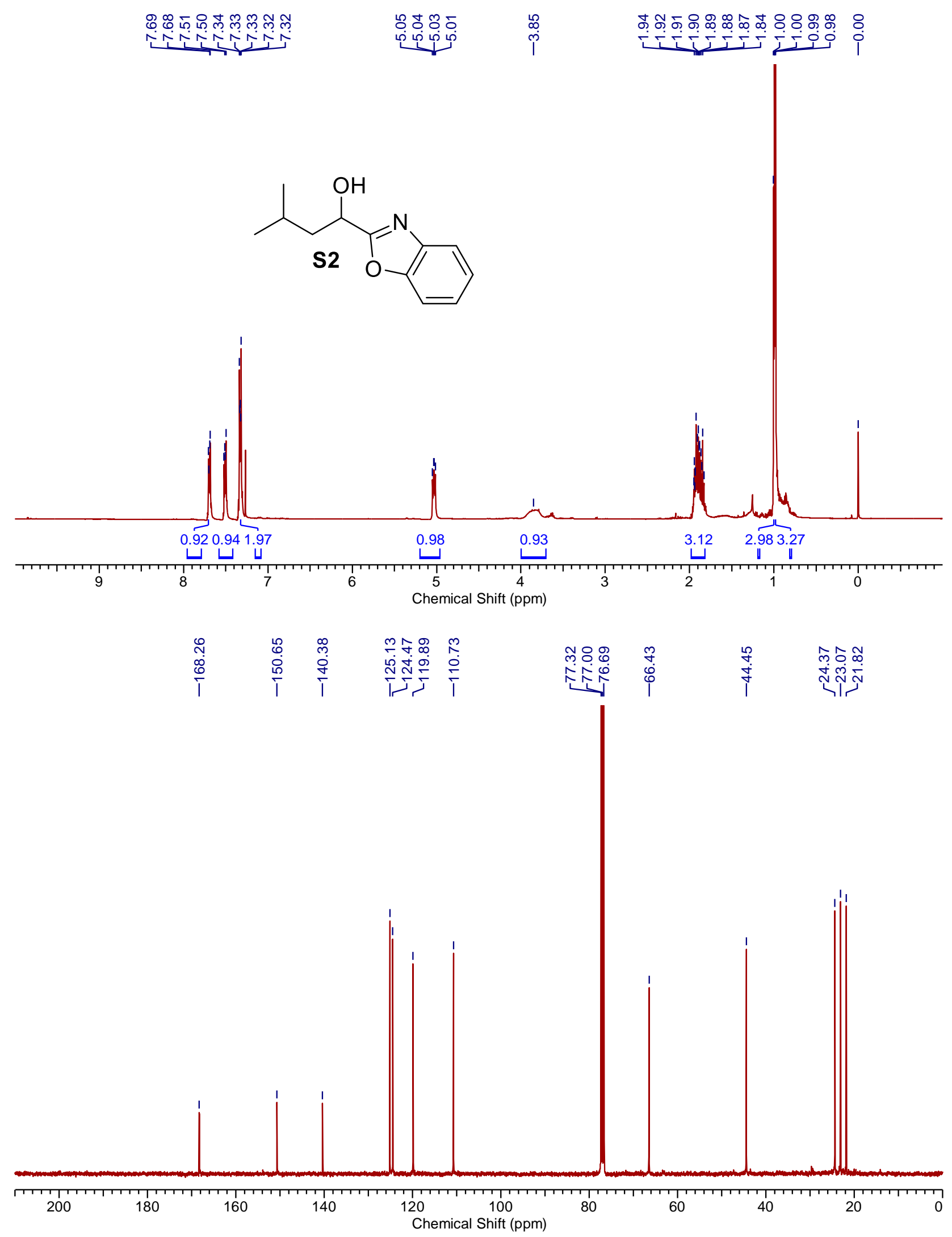




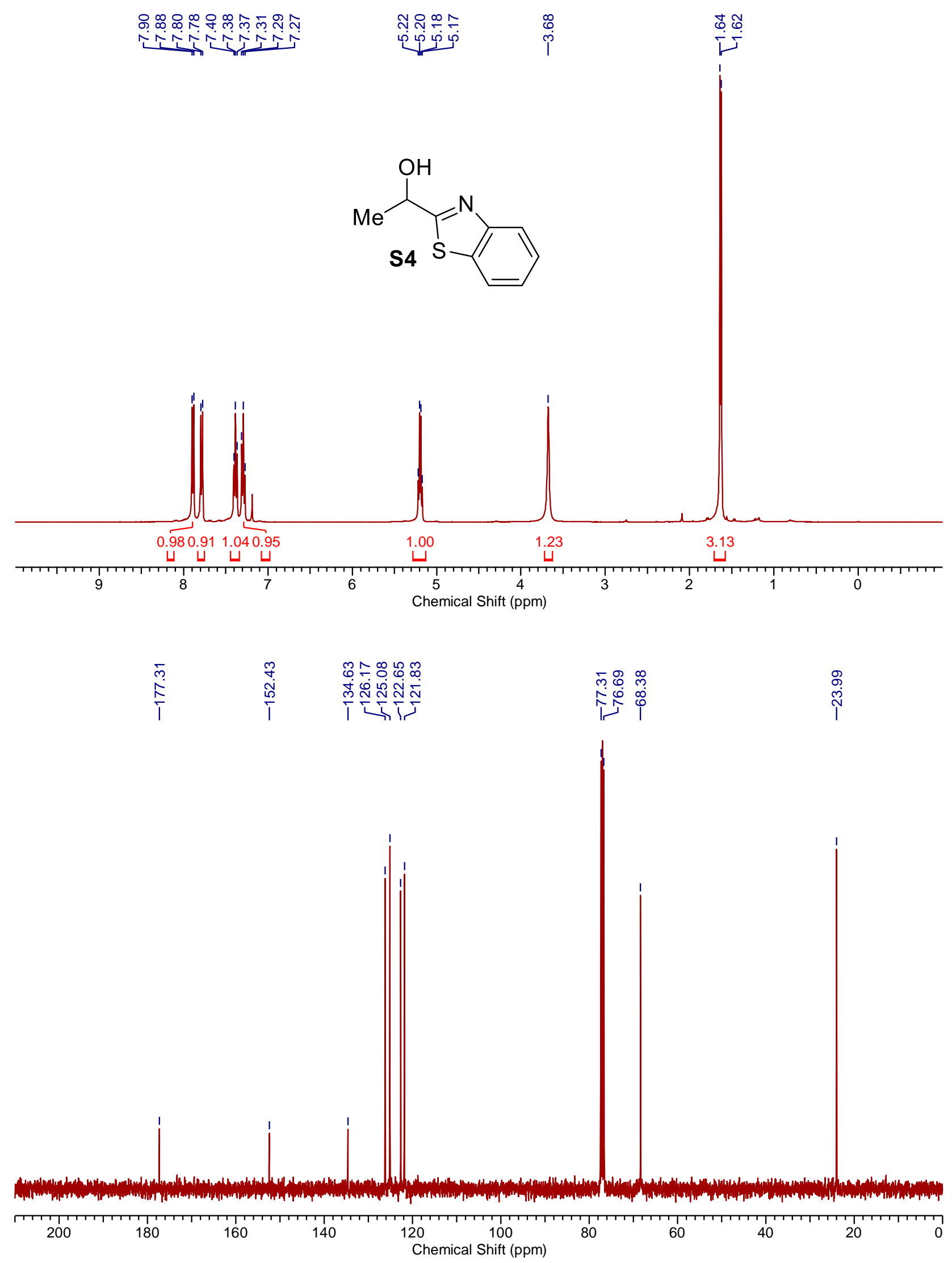




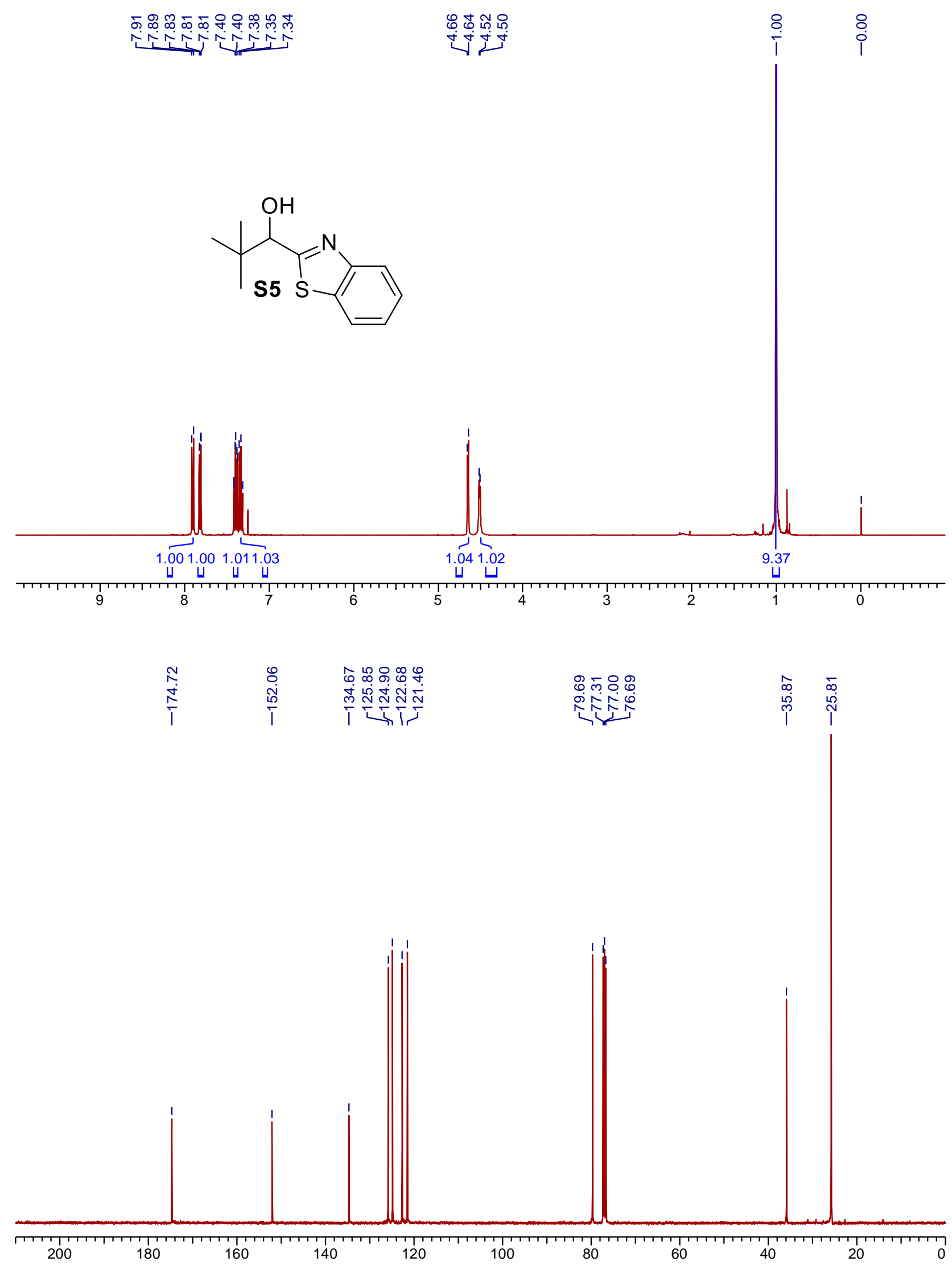



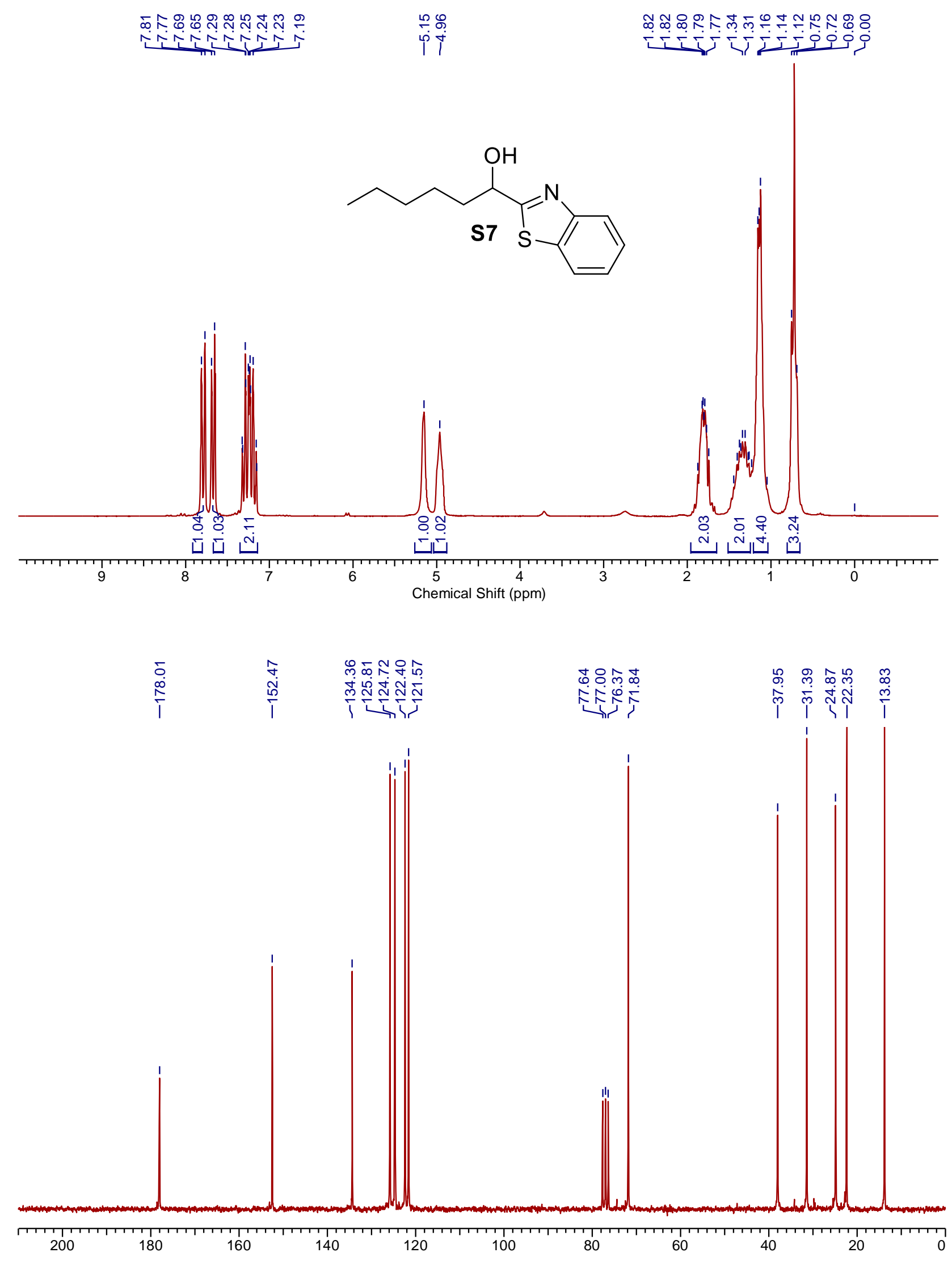

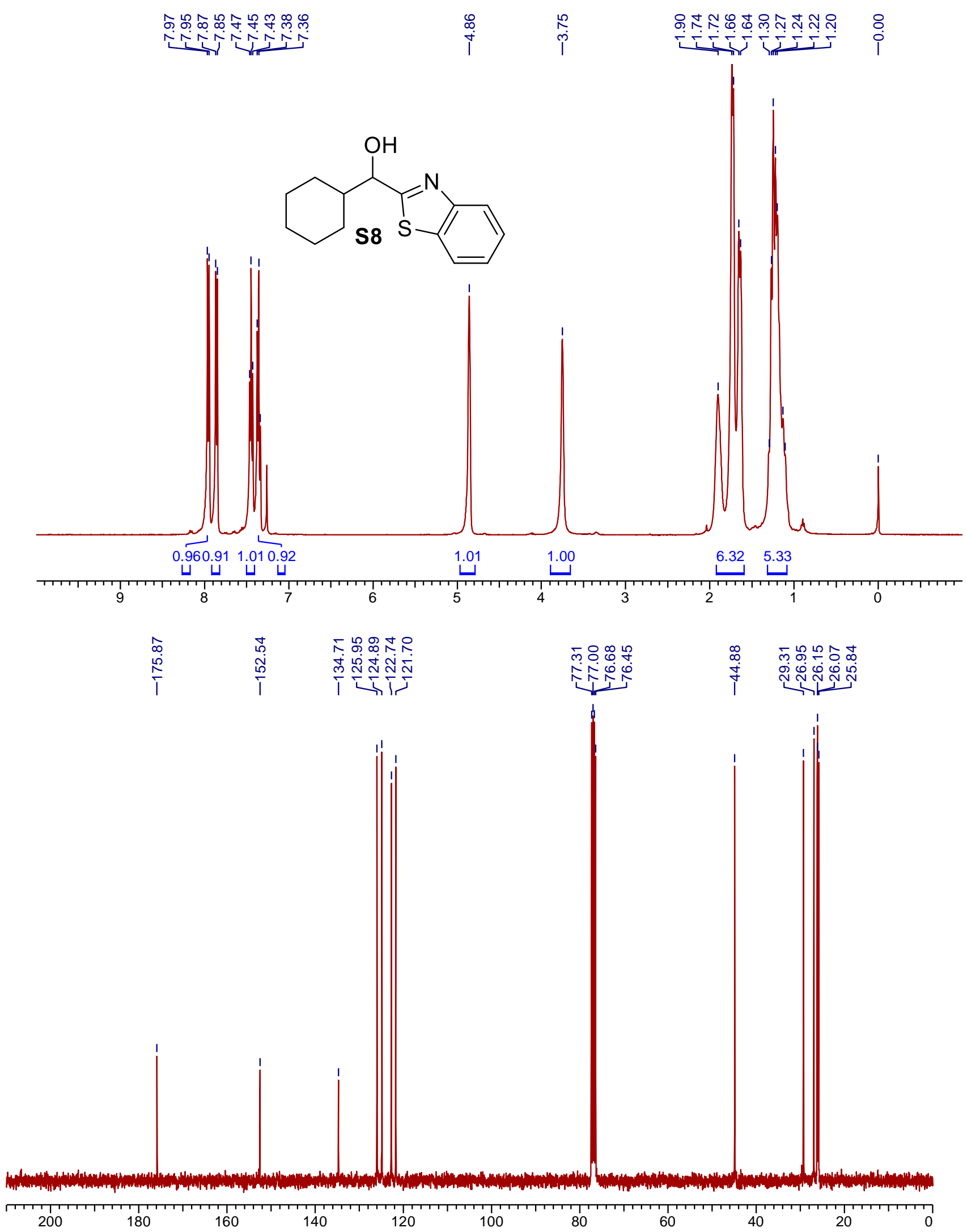

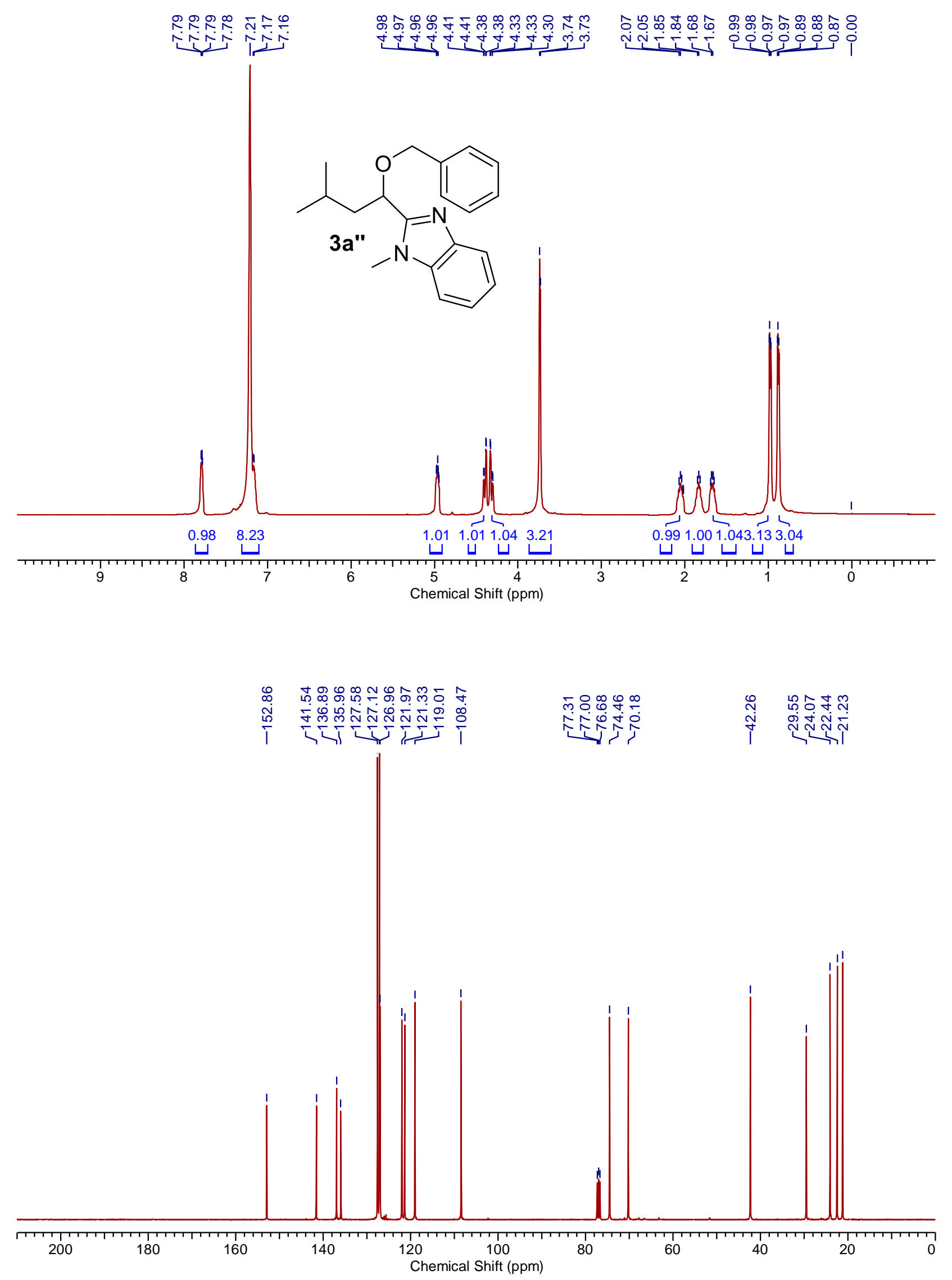

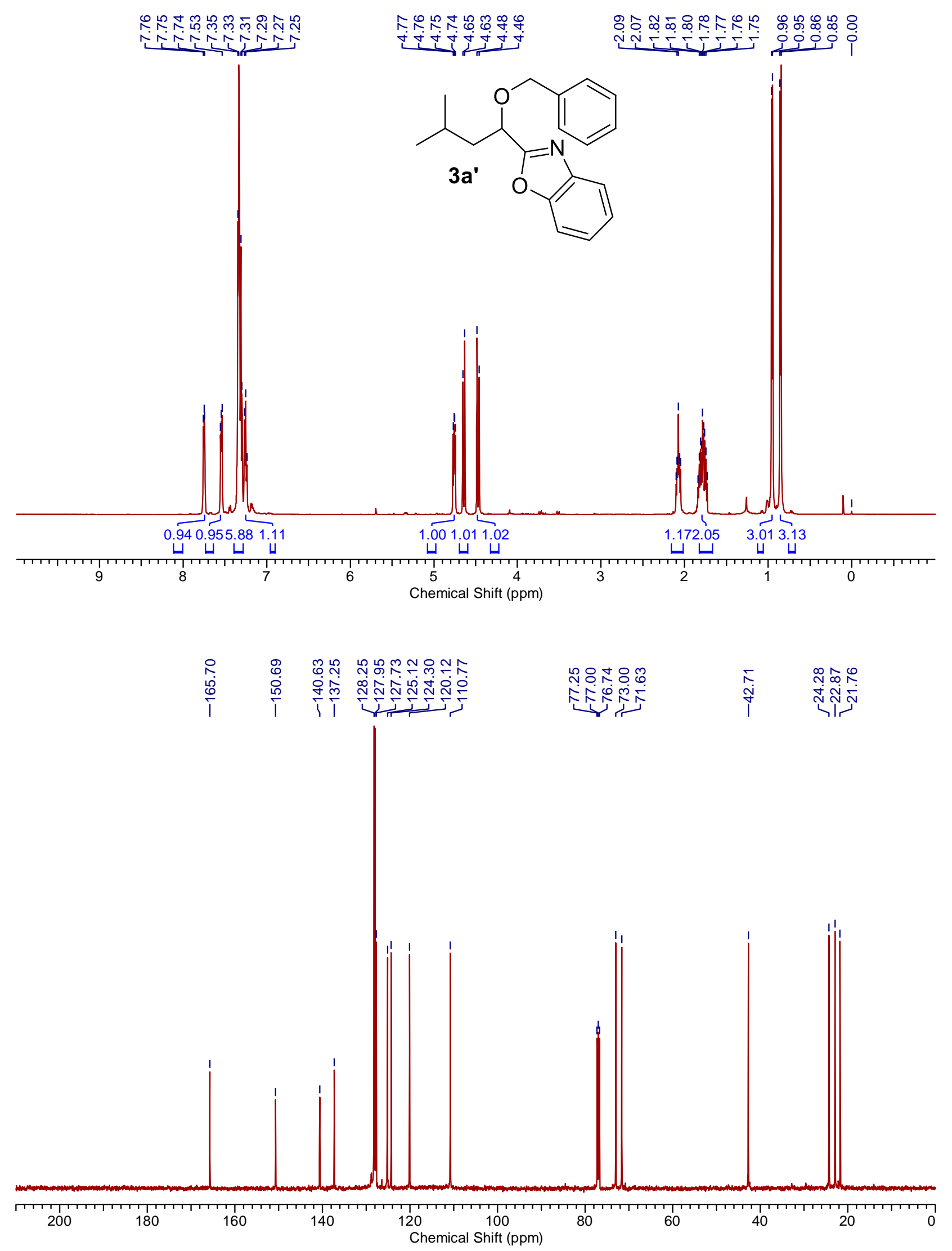

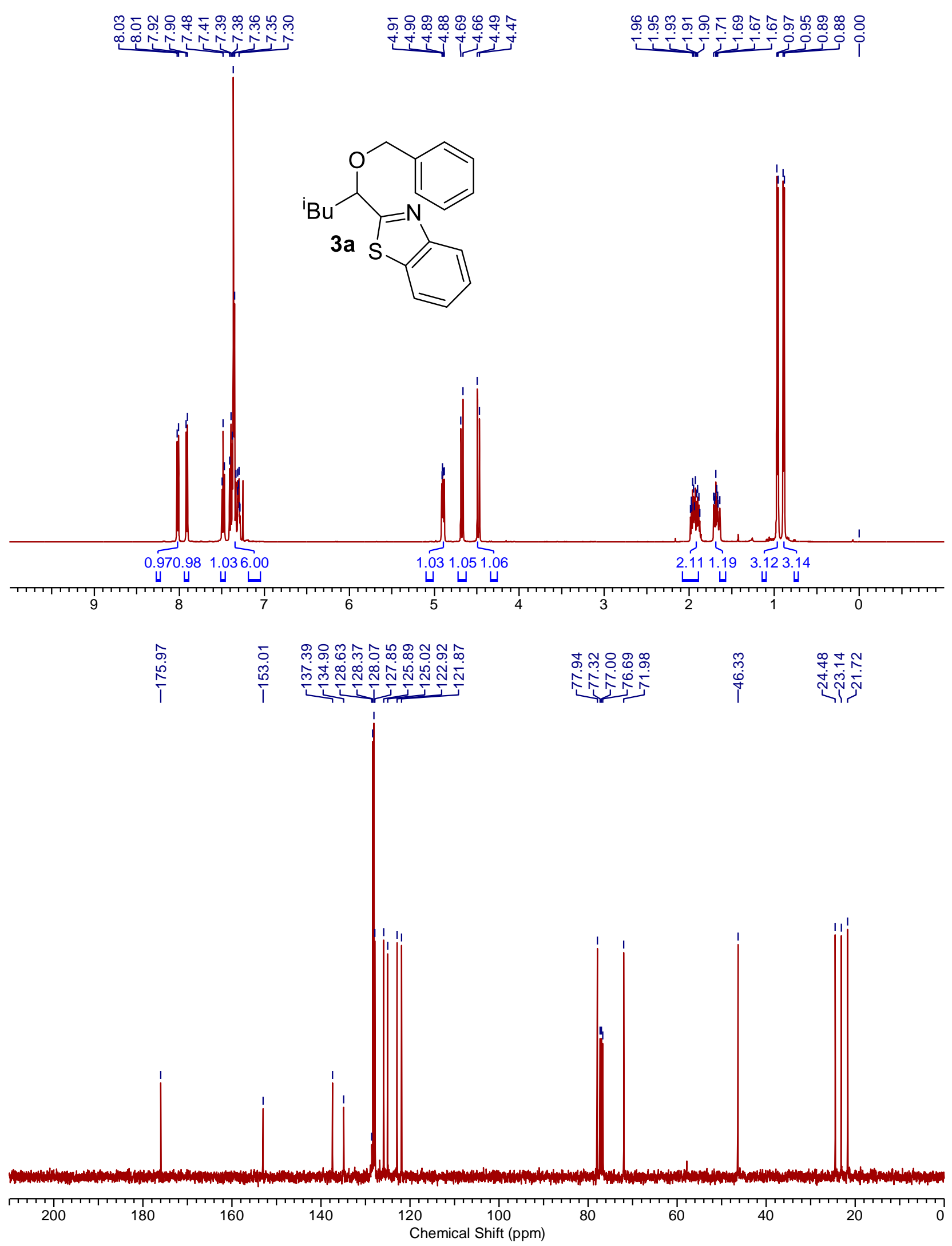

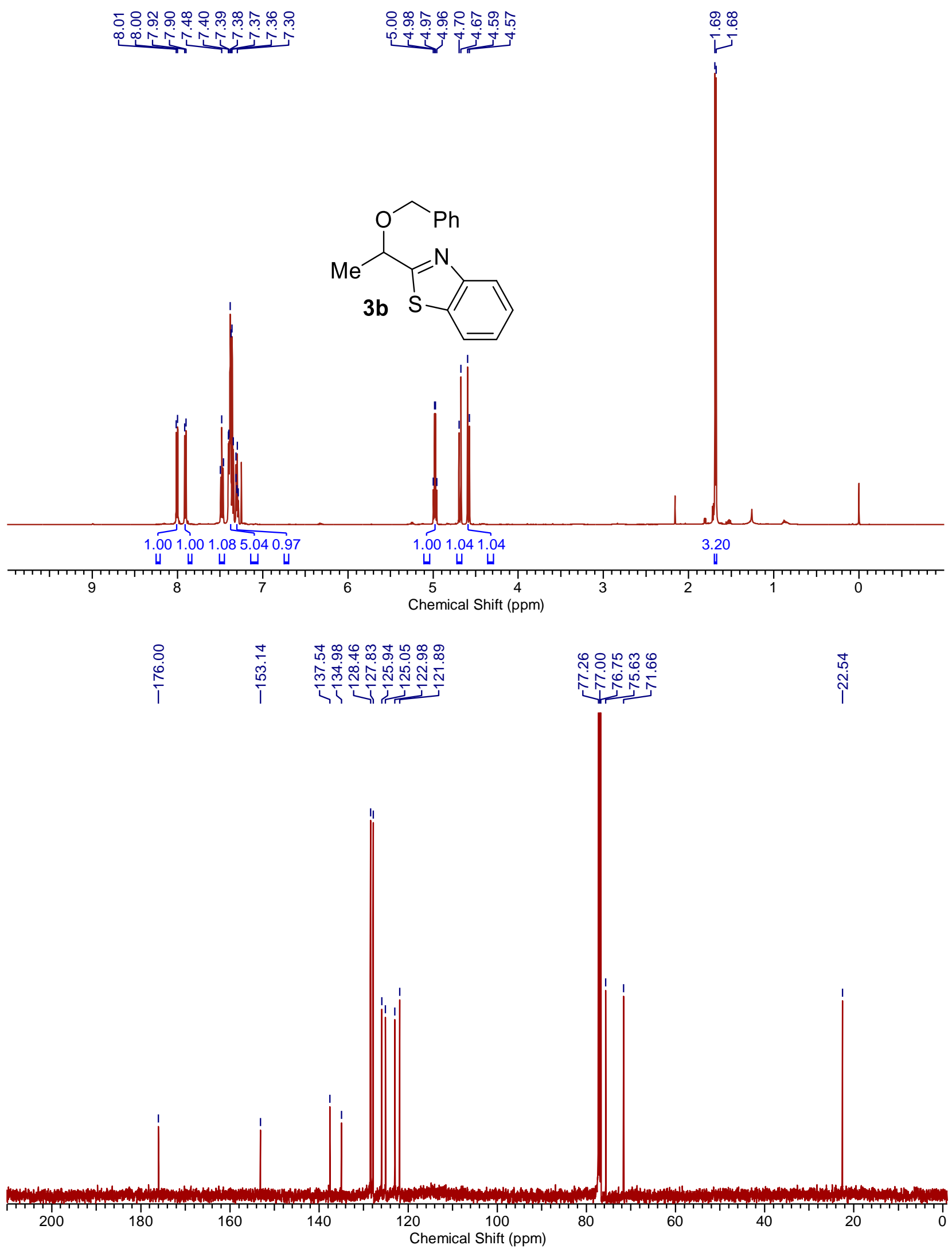


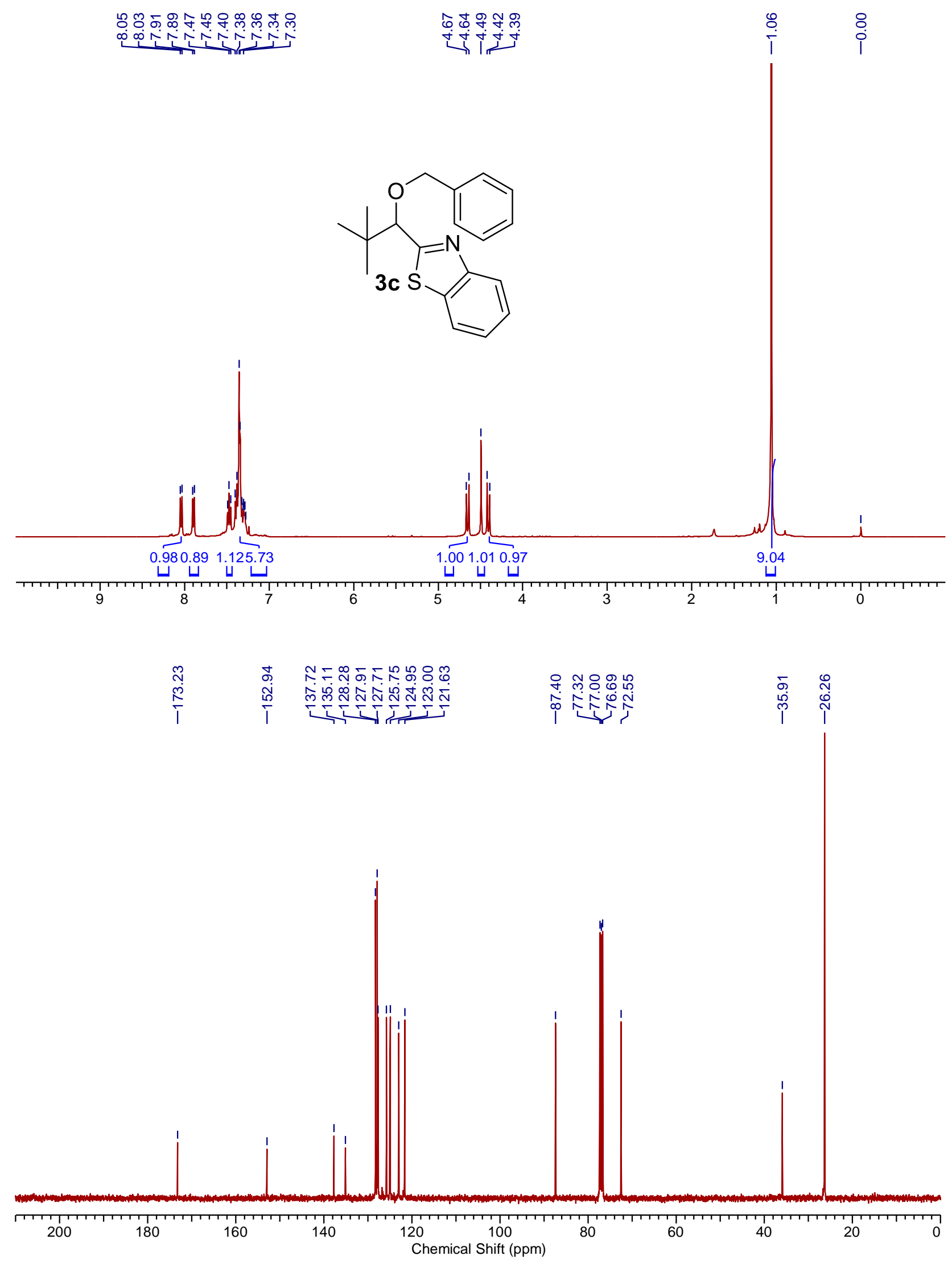



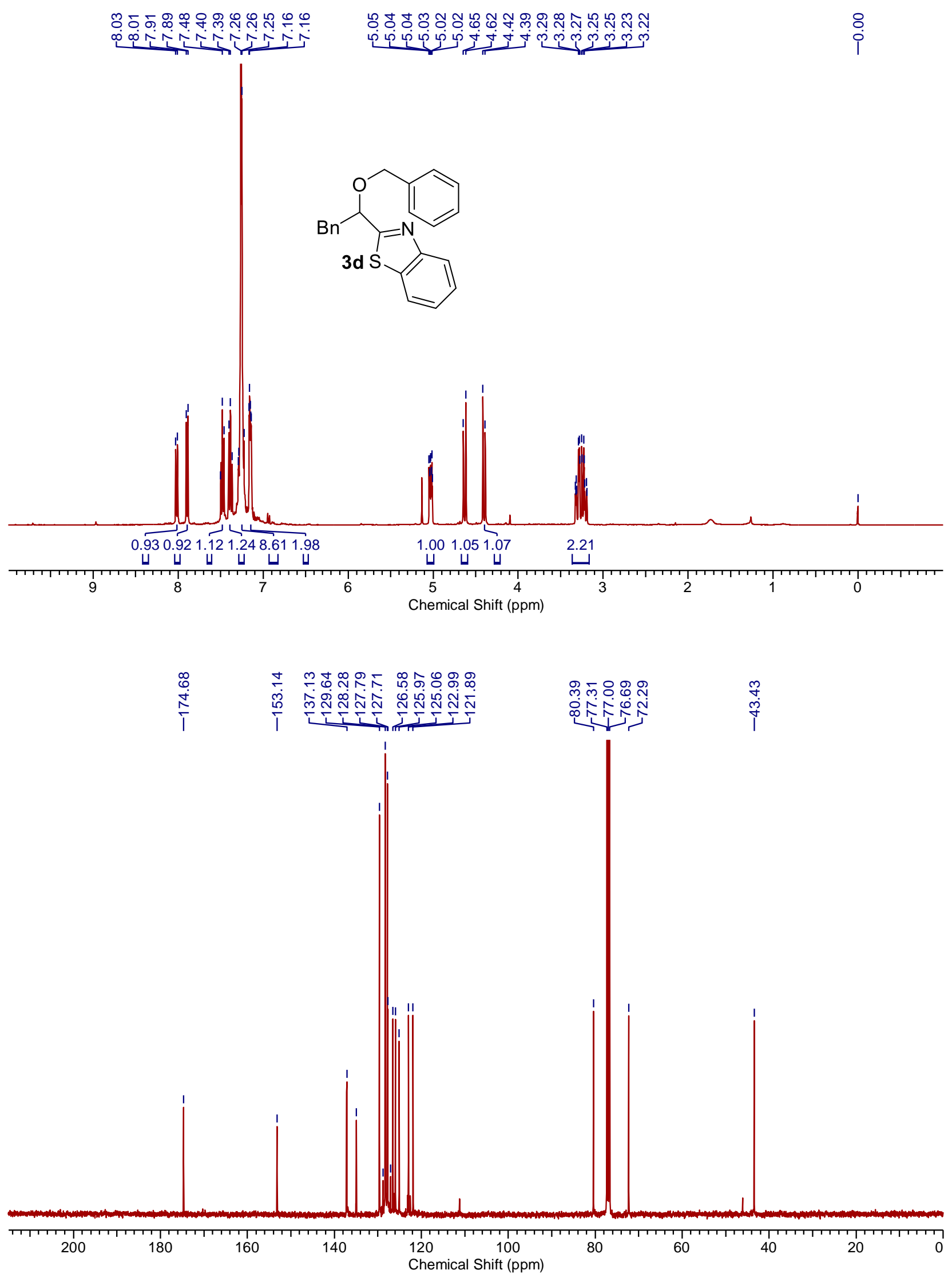

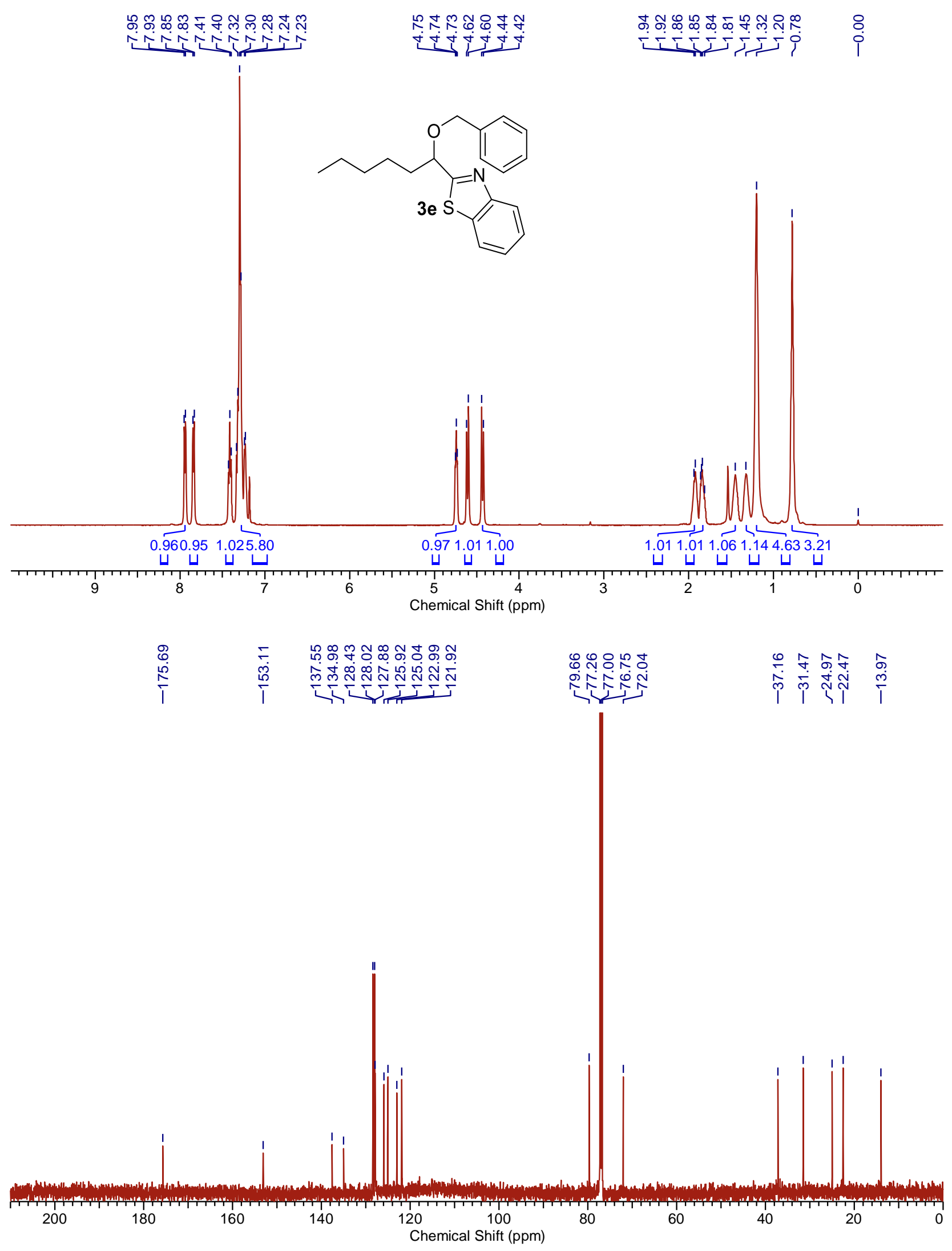

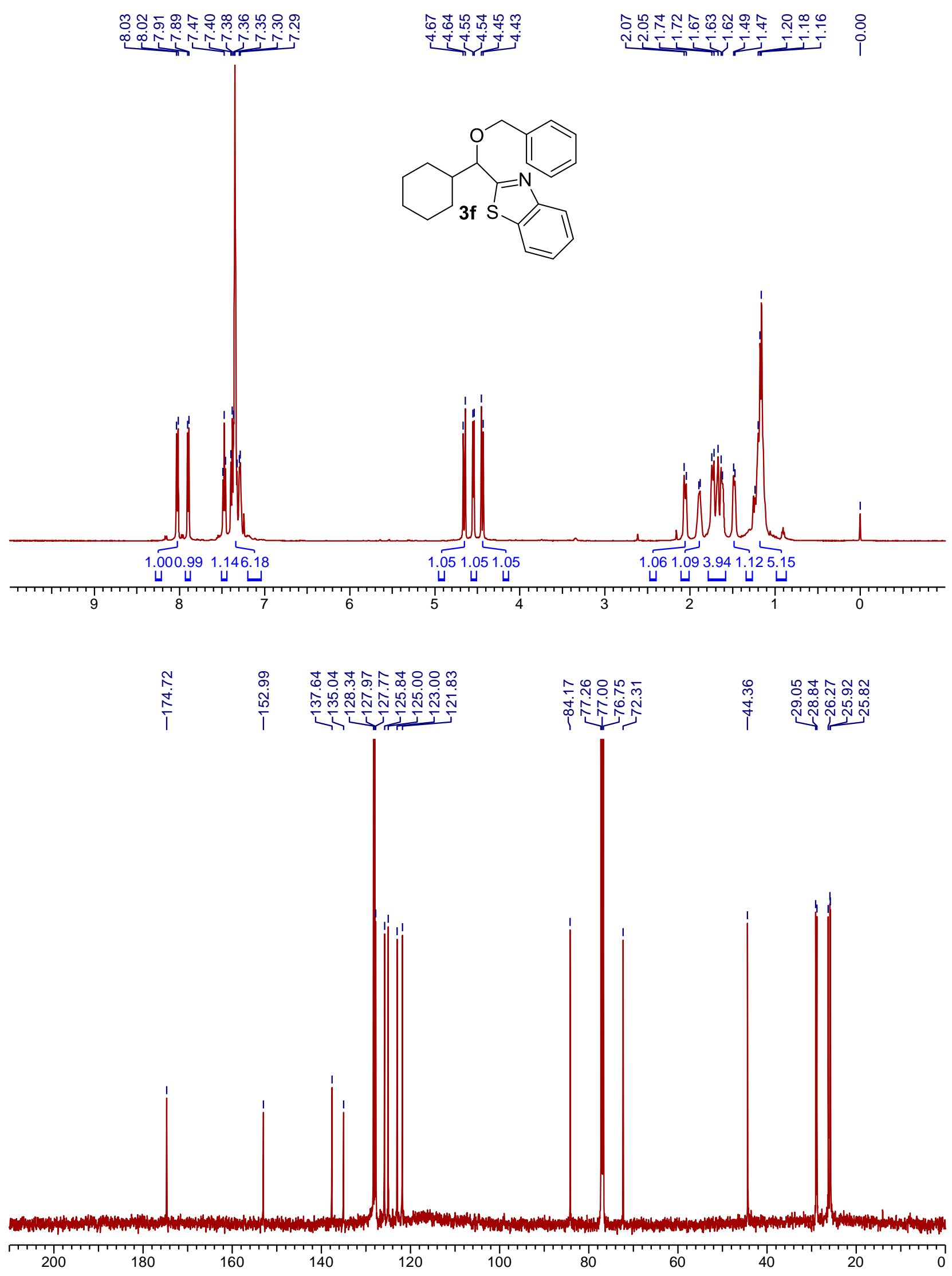

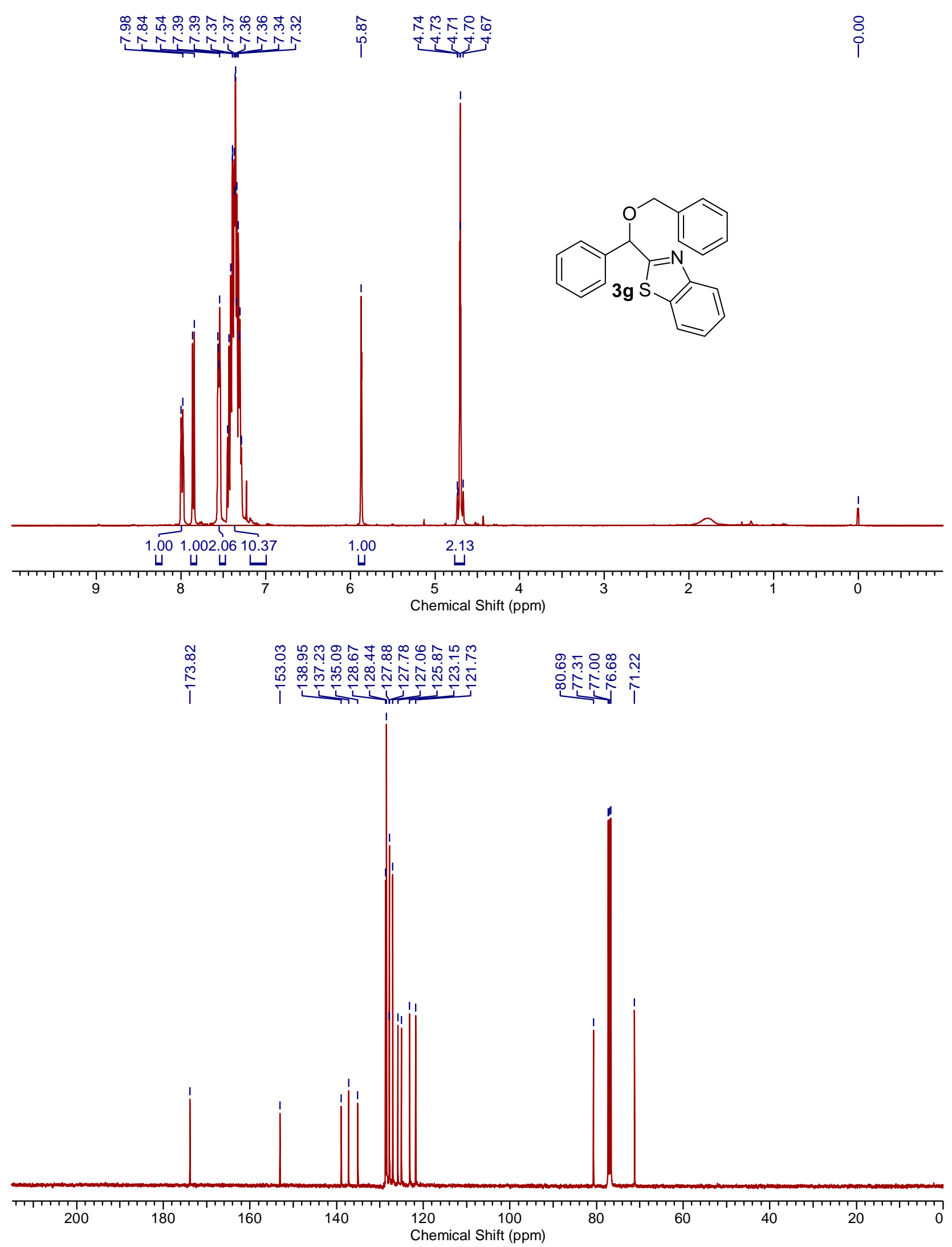


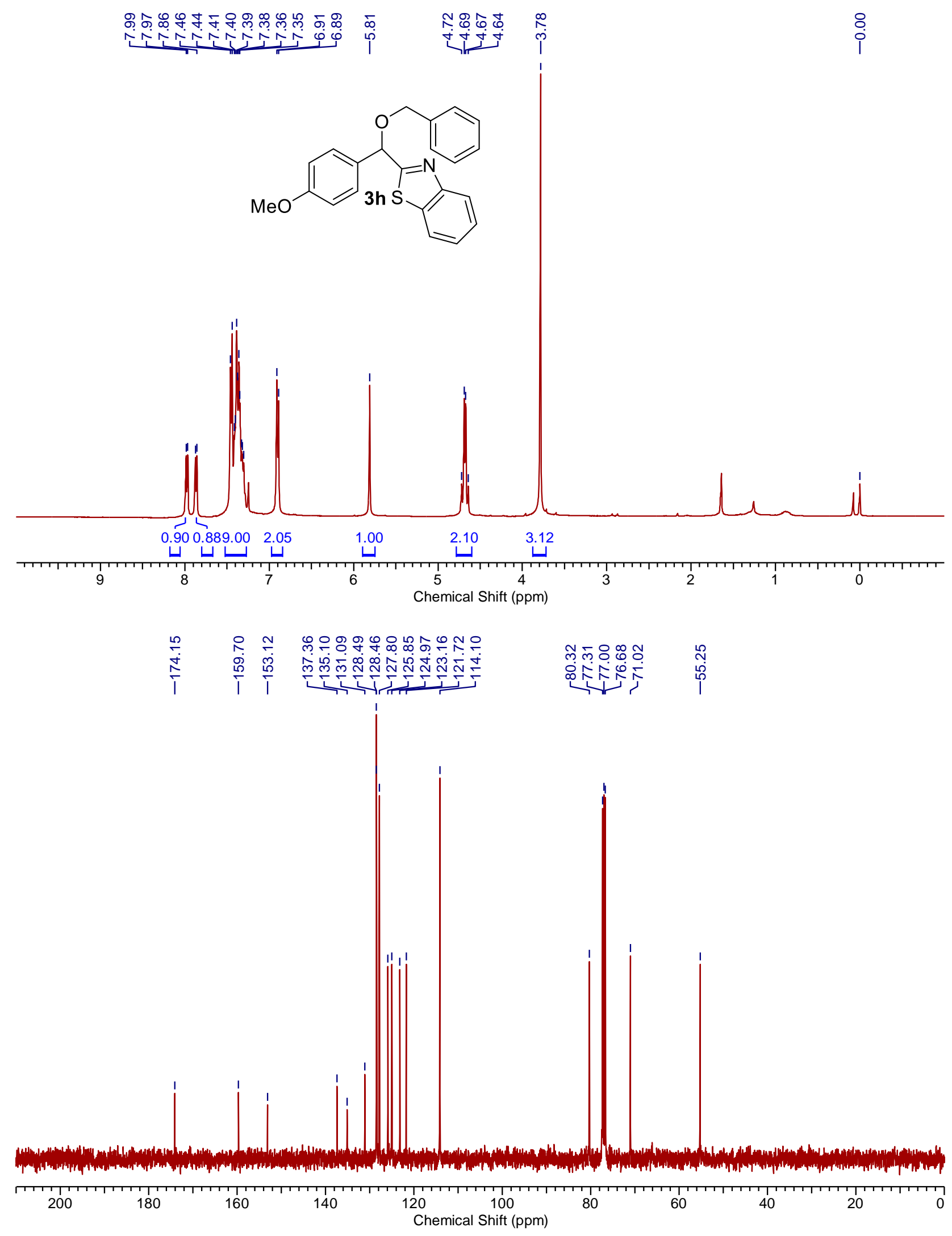



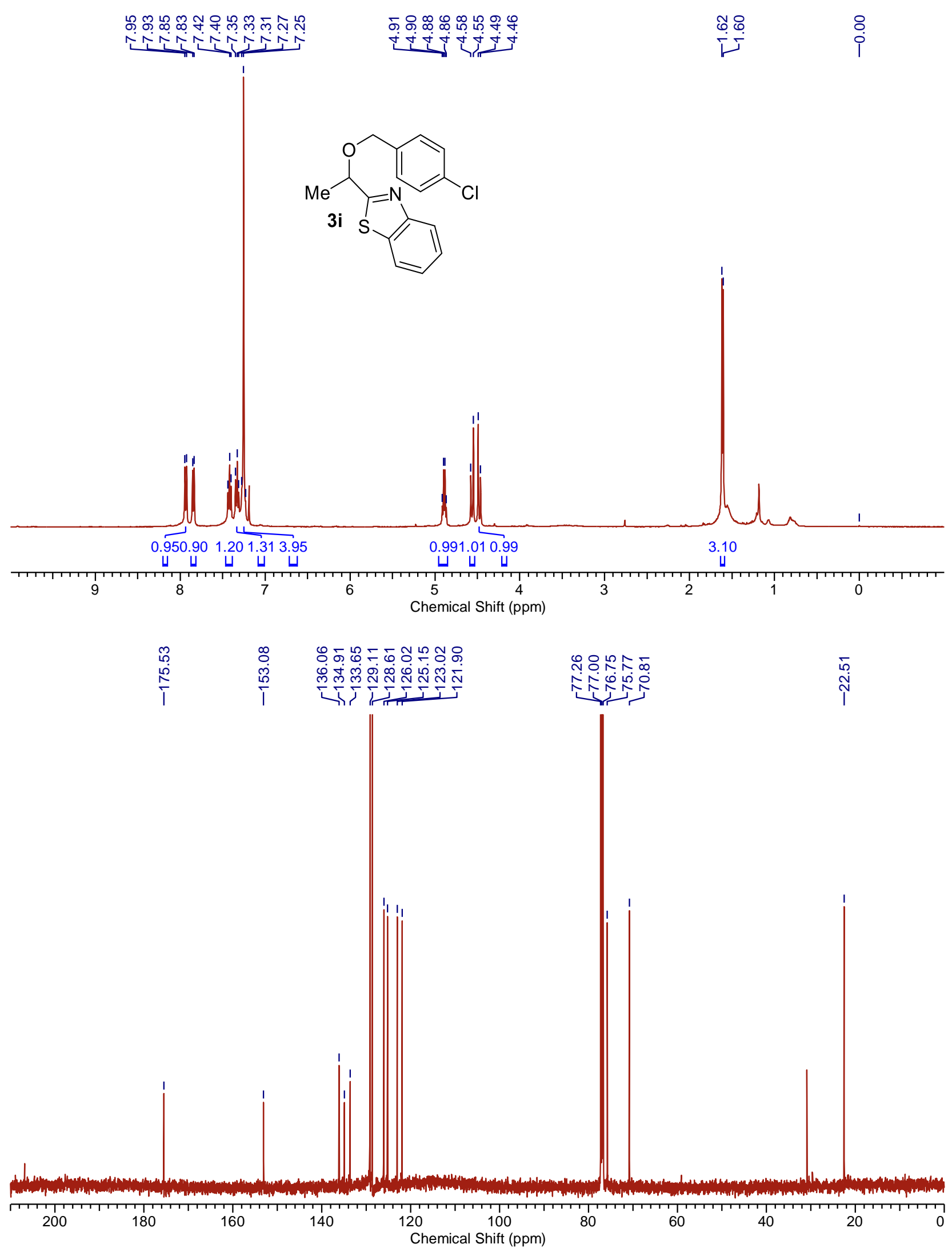
<smiles>CC(OCc1ccc(F)cc1)c1nc2ccccc2s1</smiles>
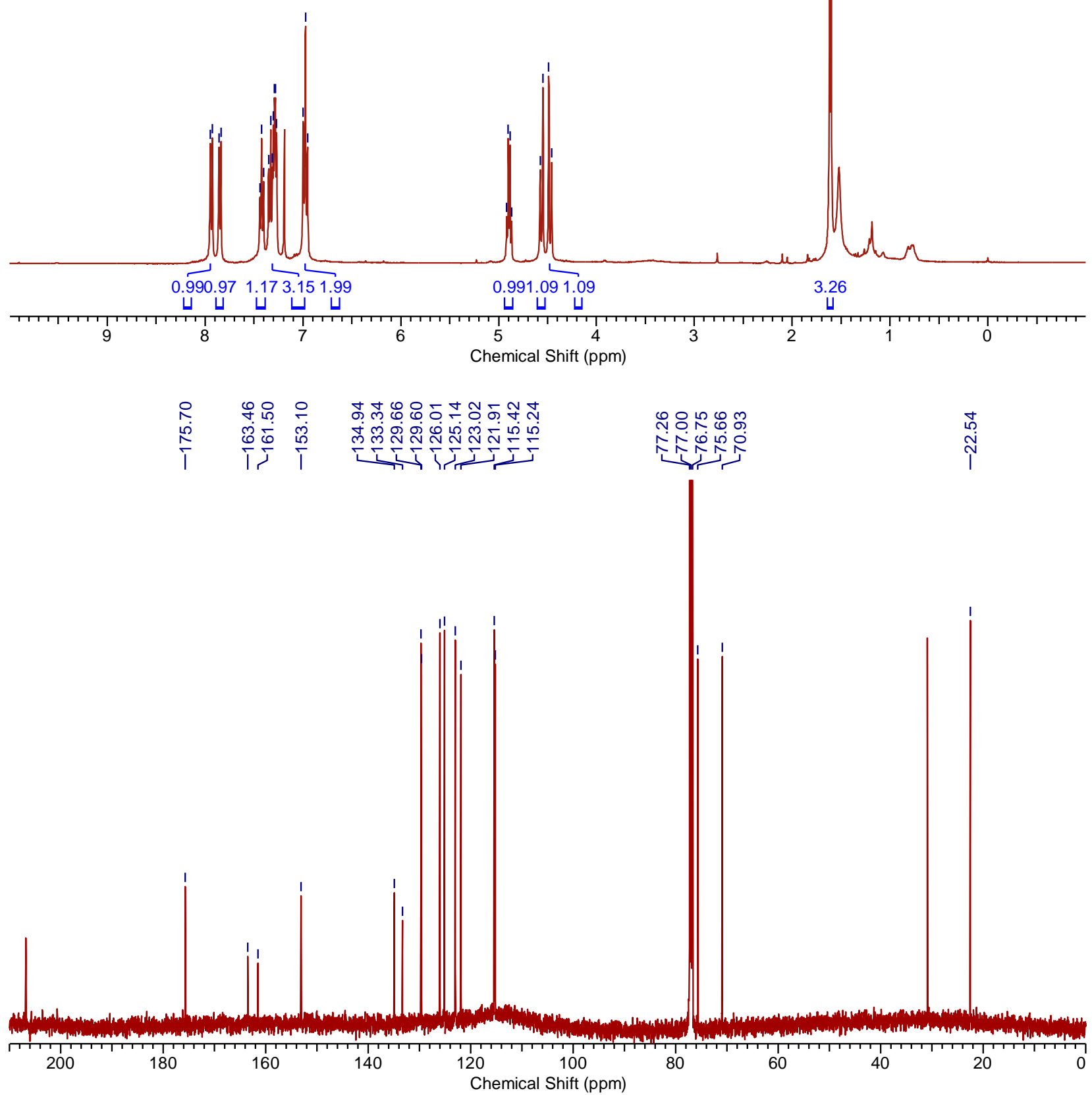

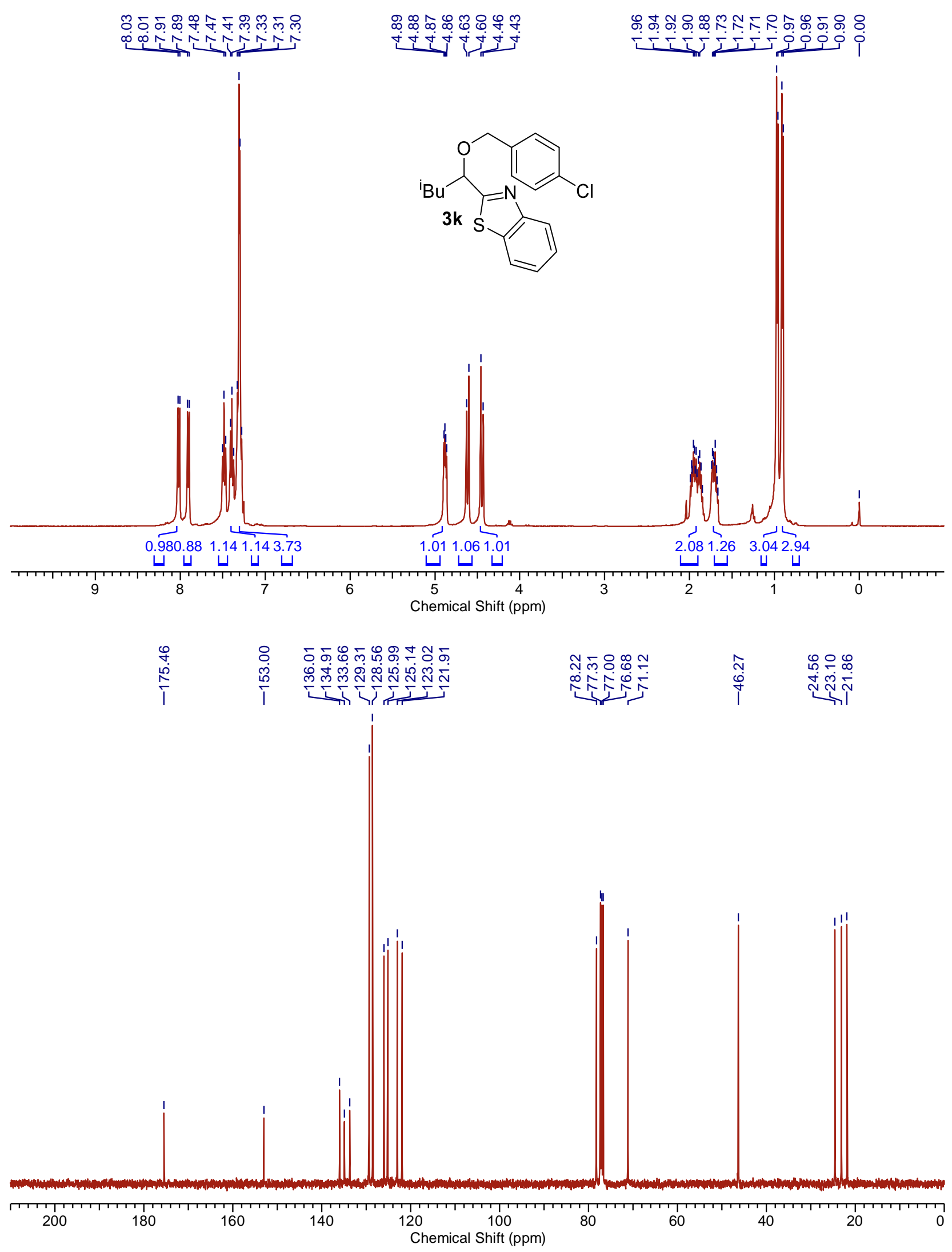


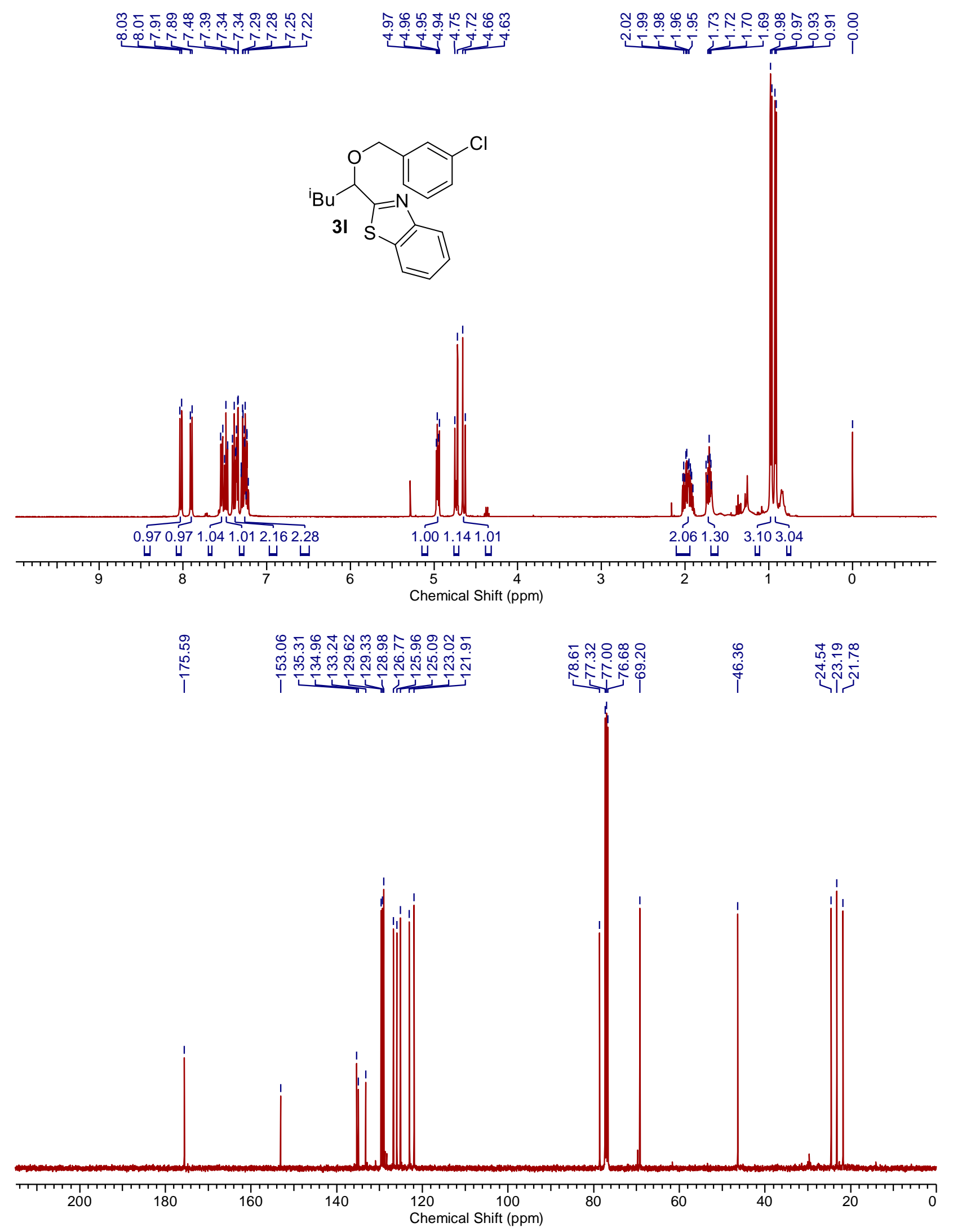




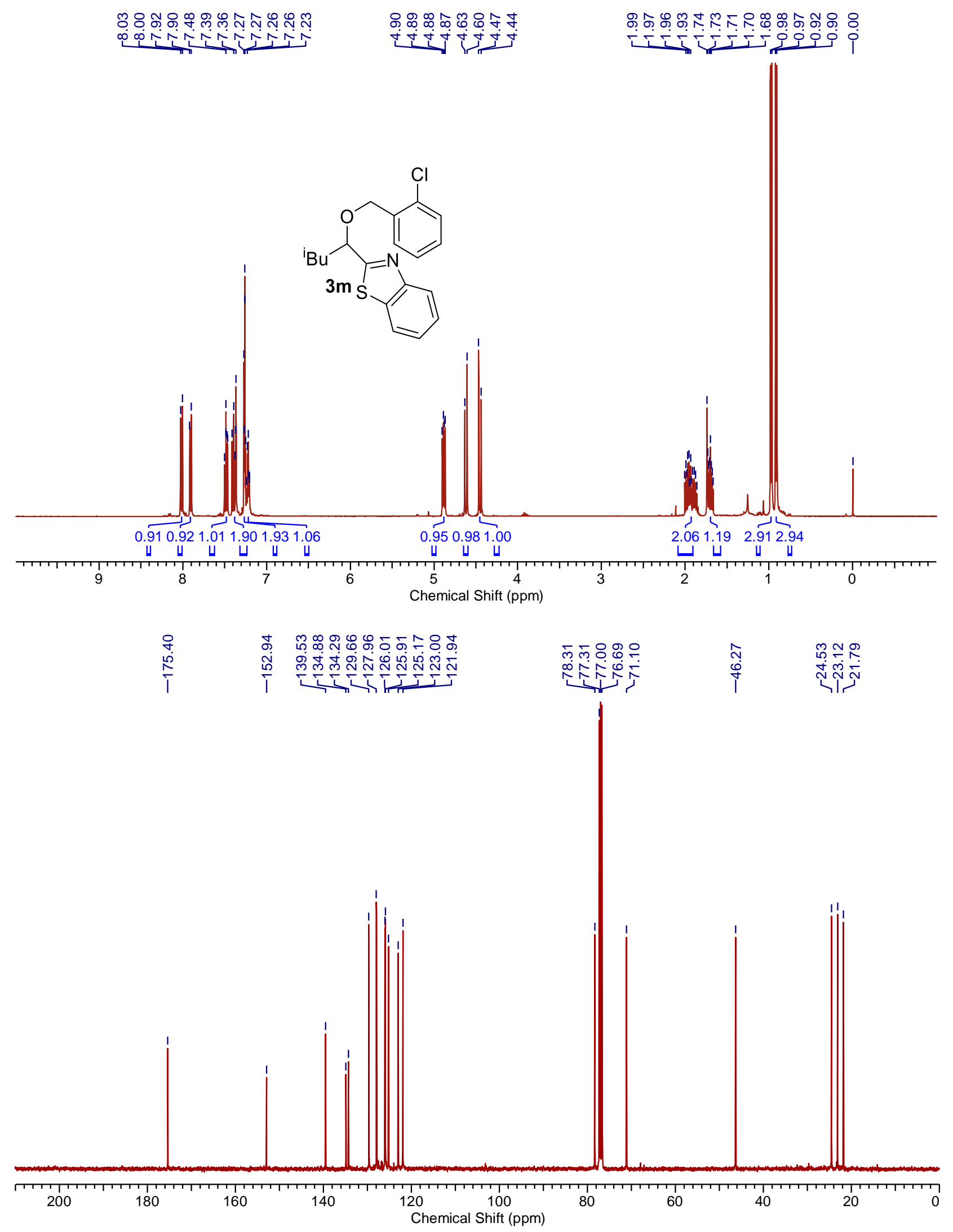



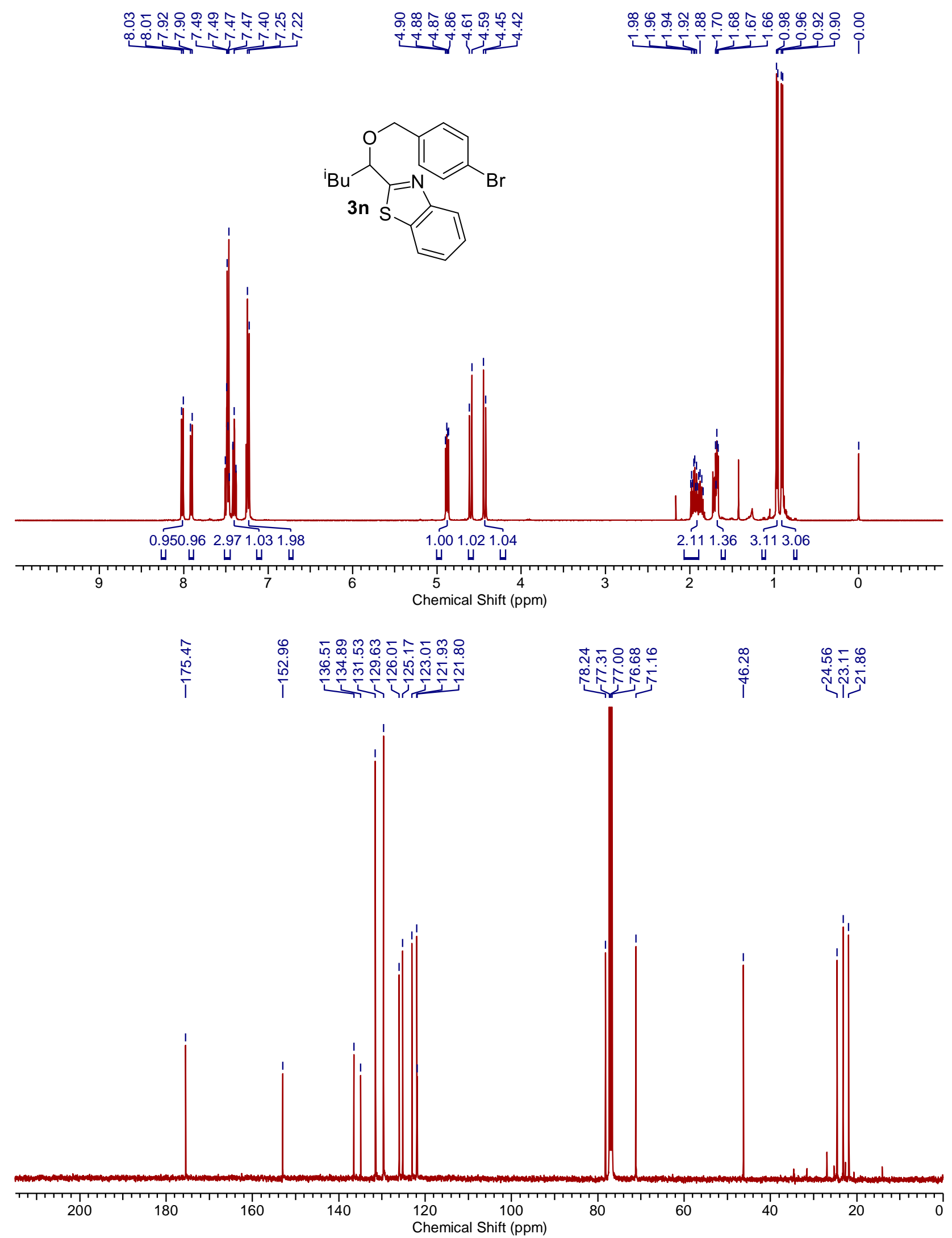


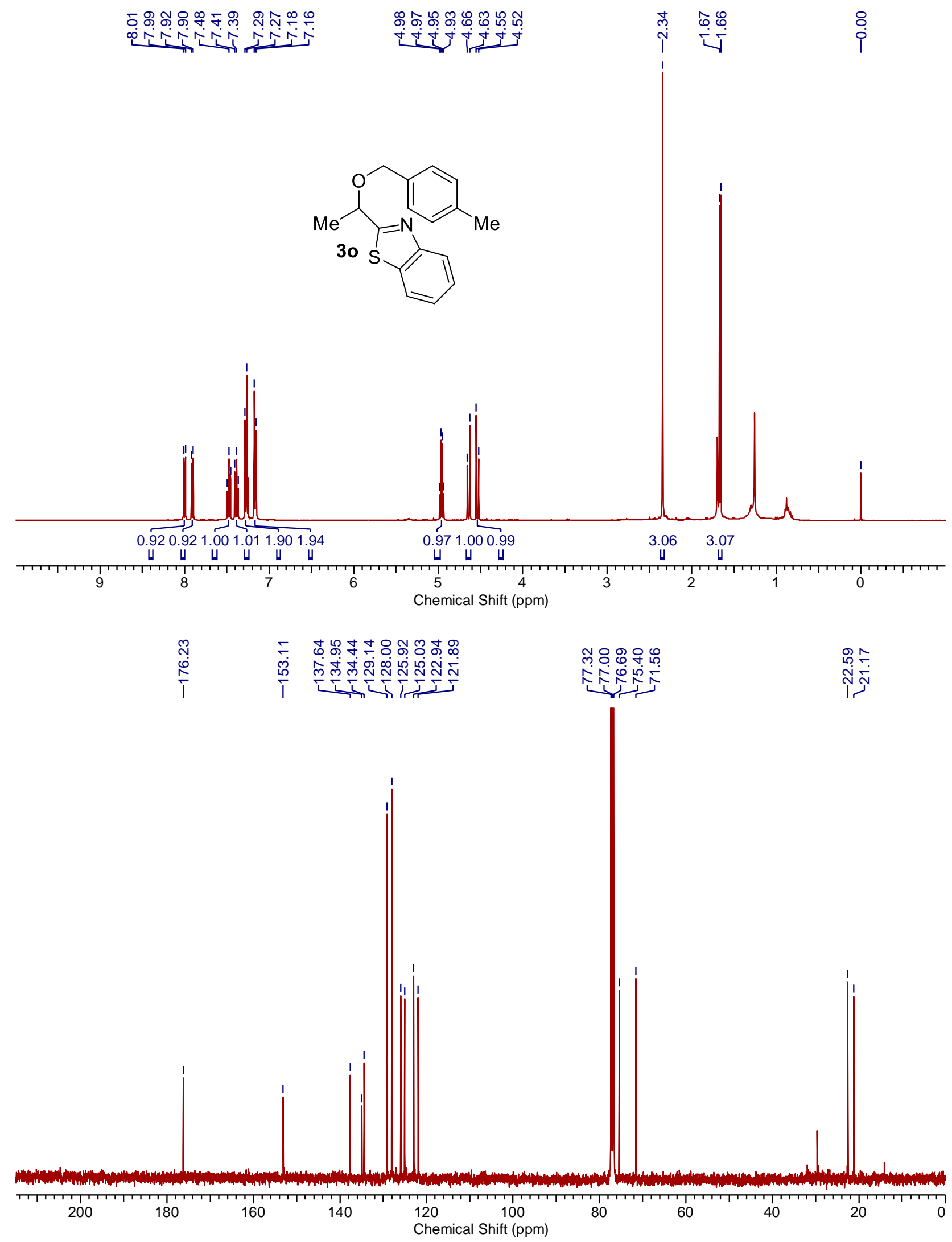



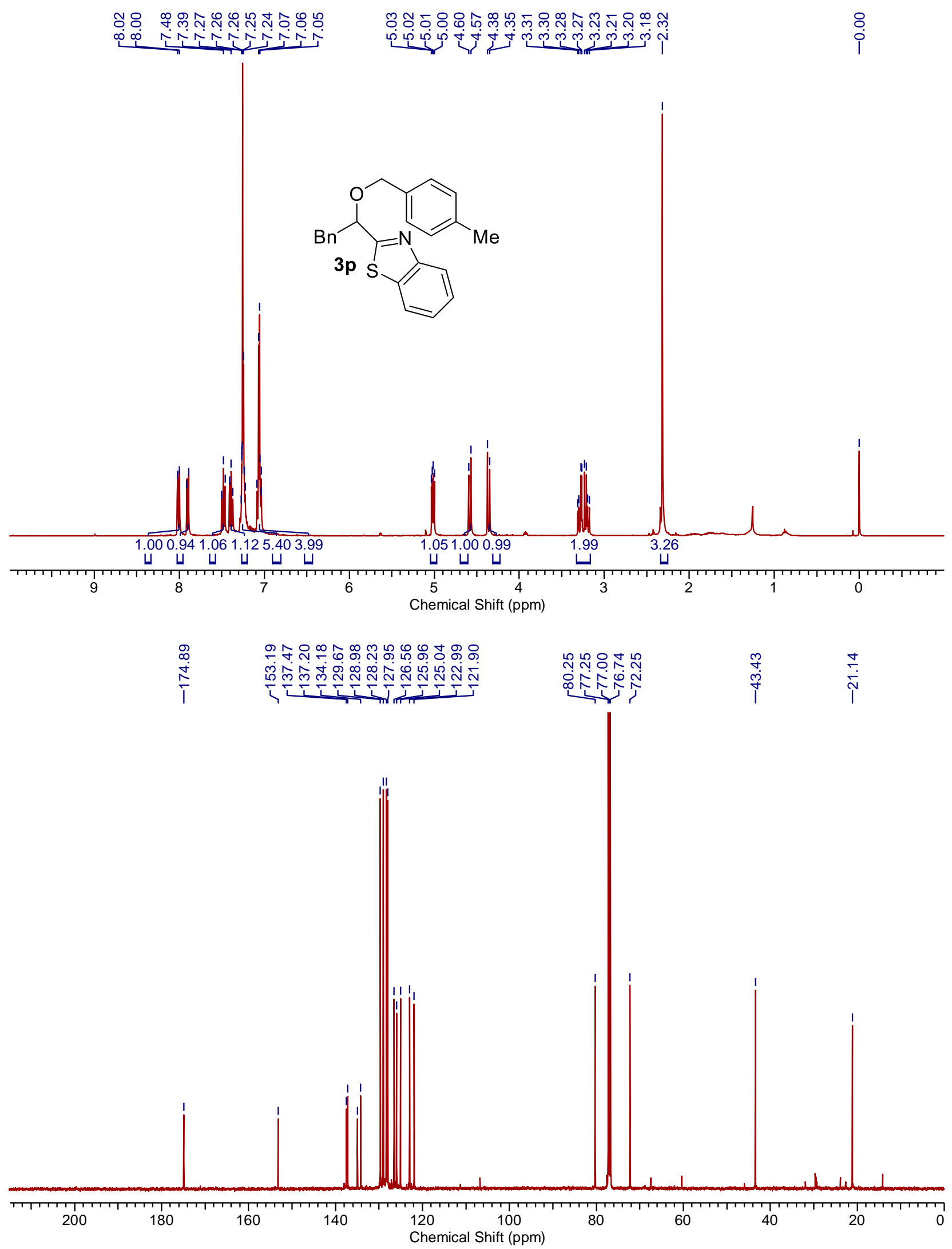

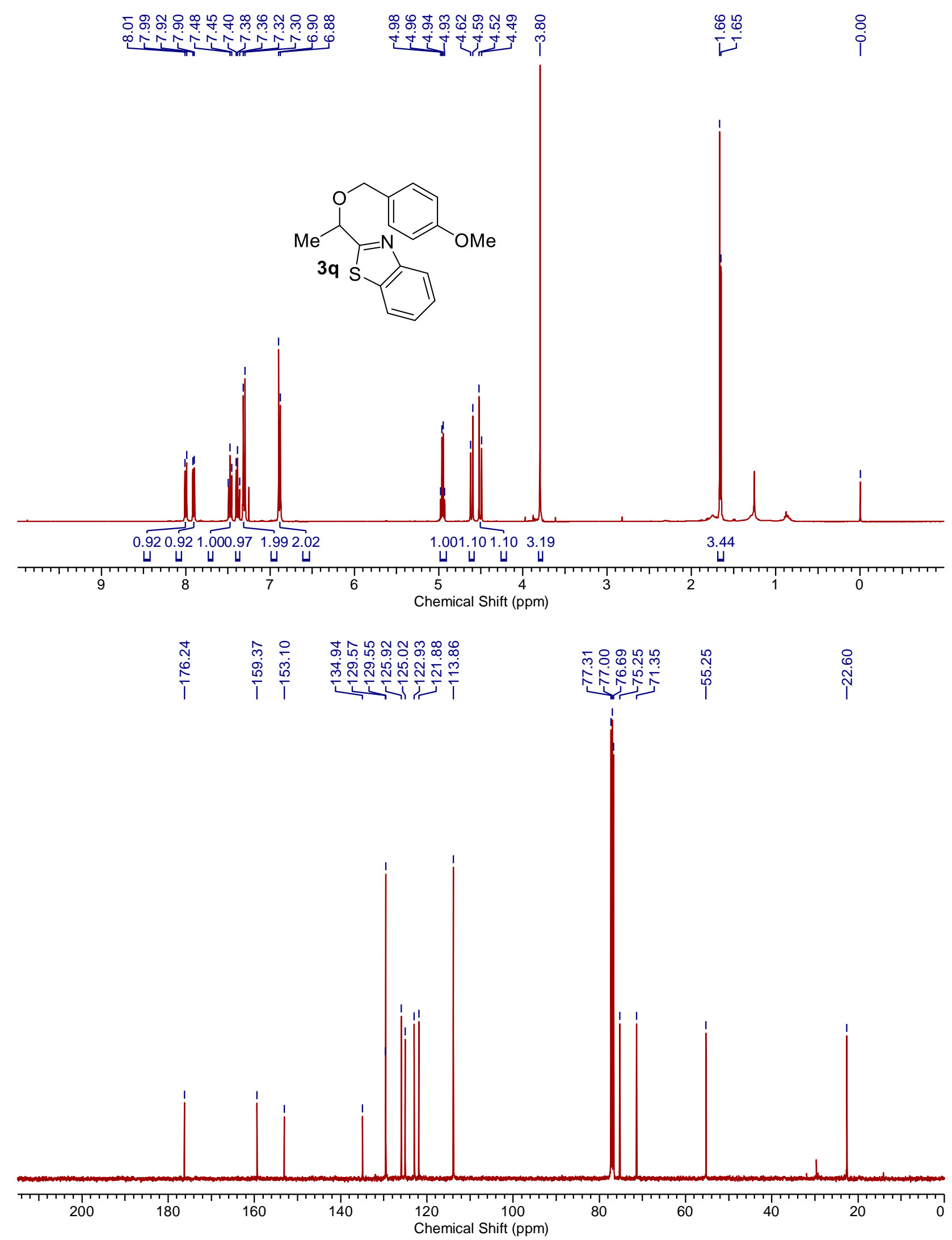


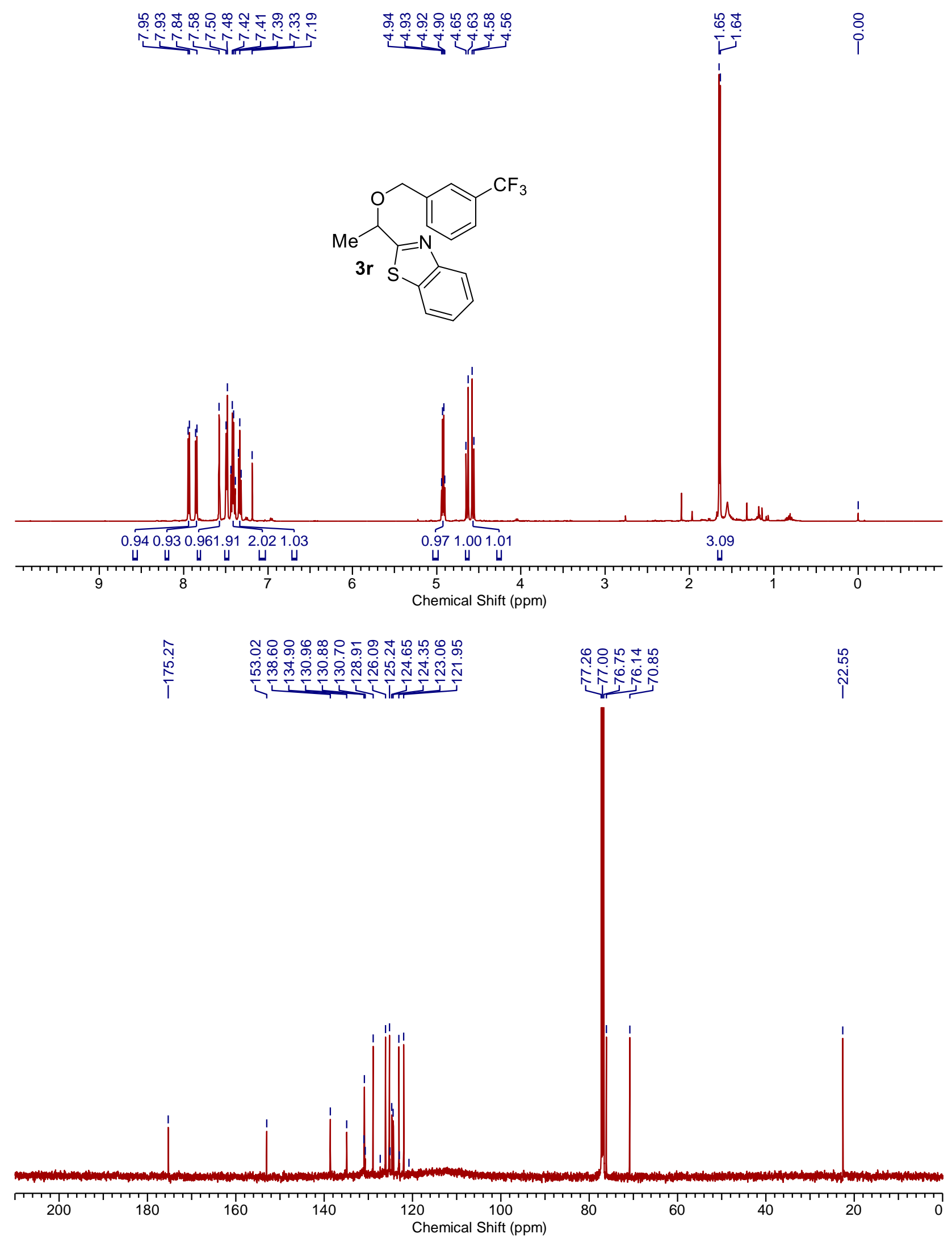




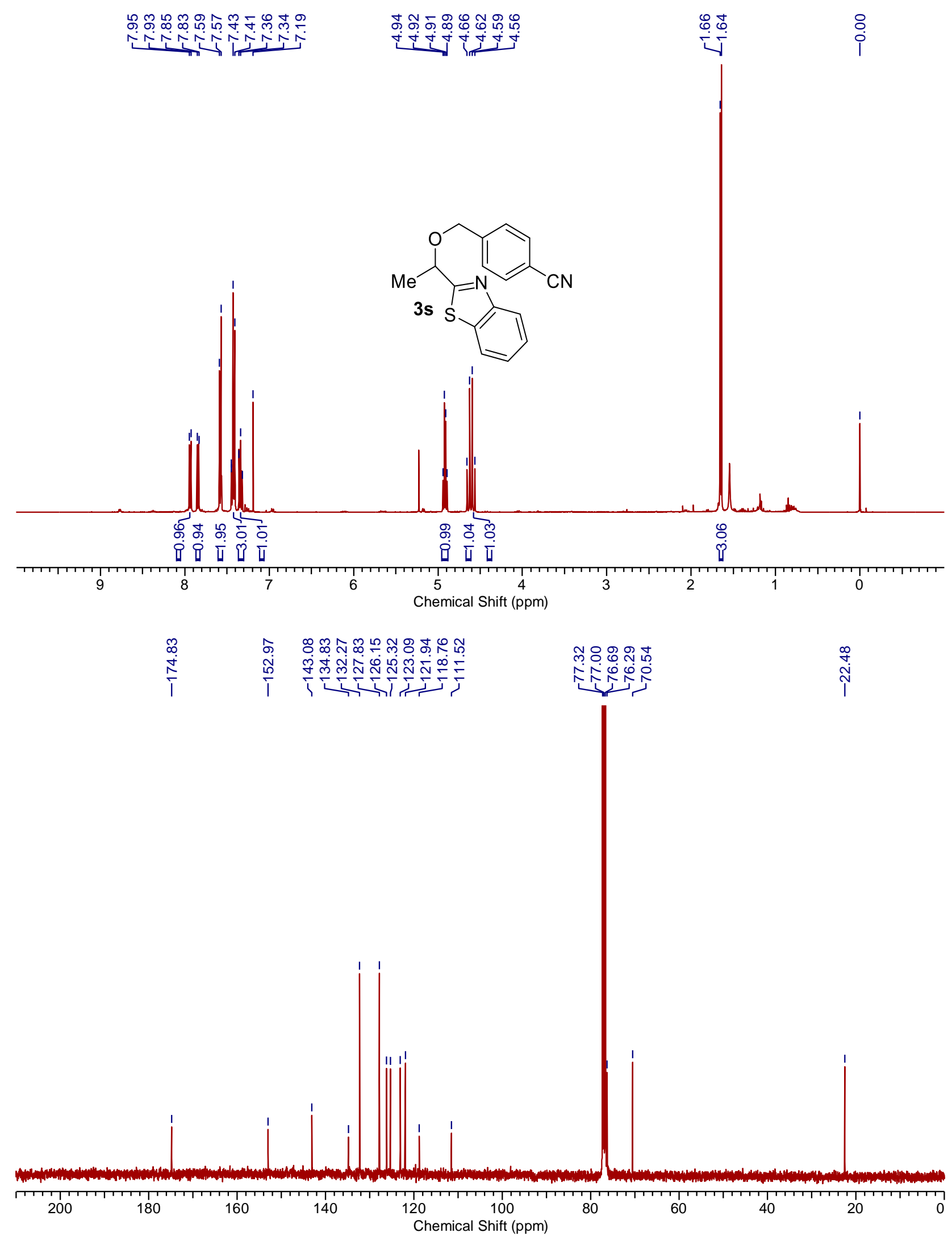



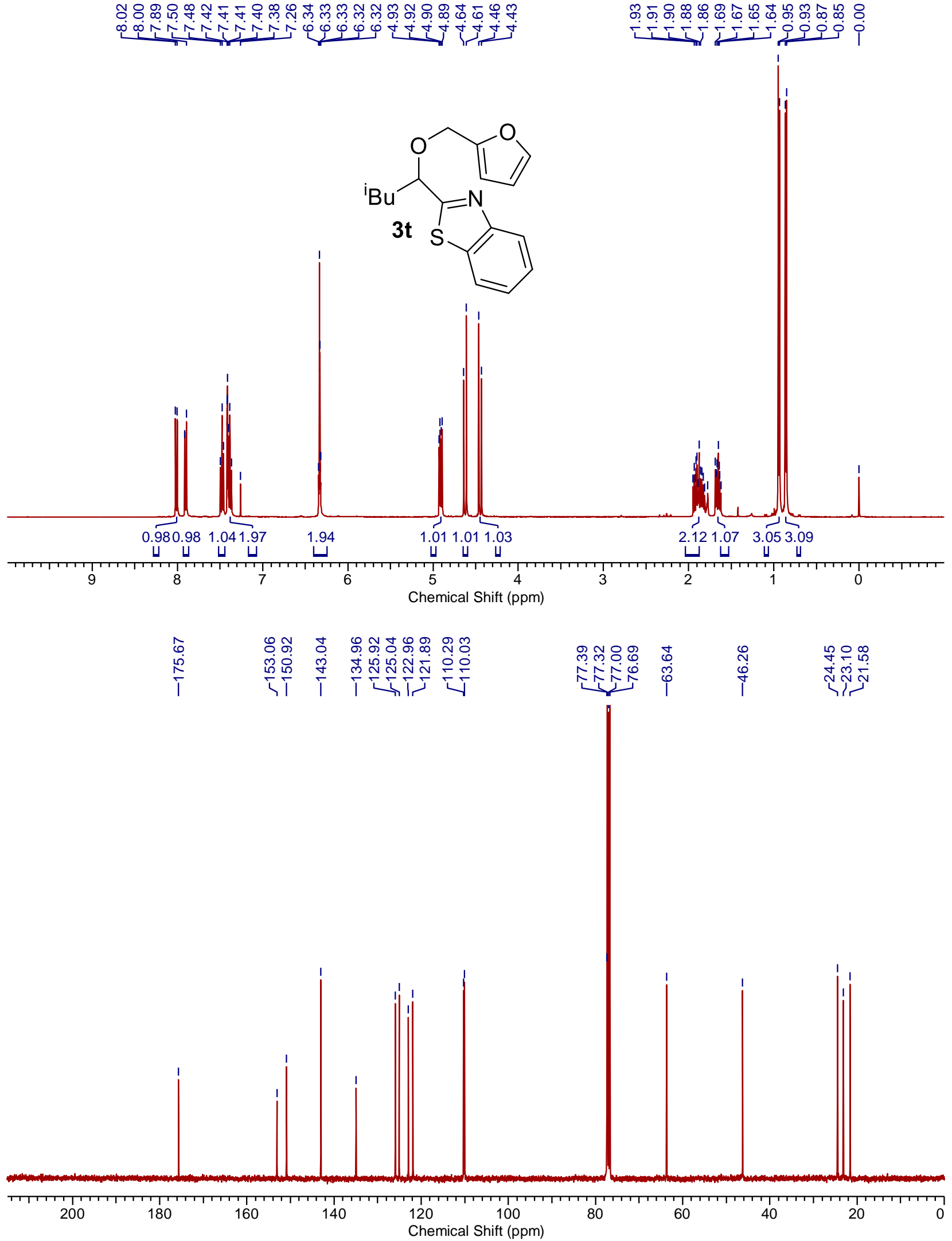

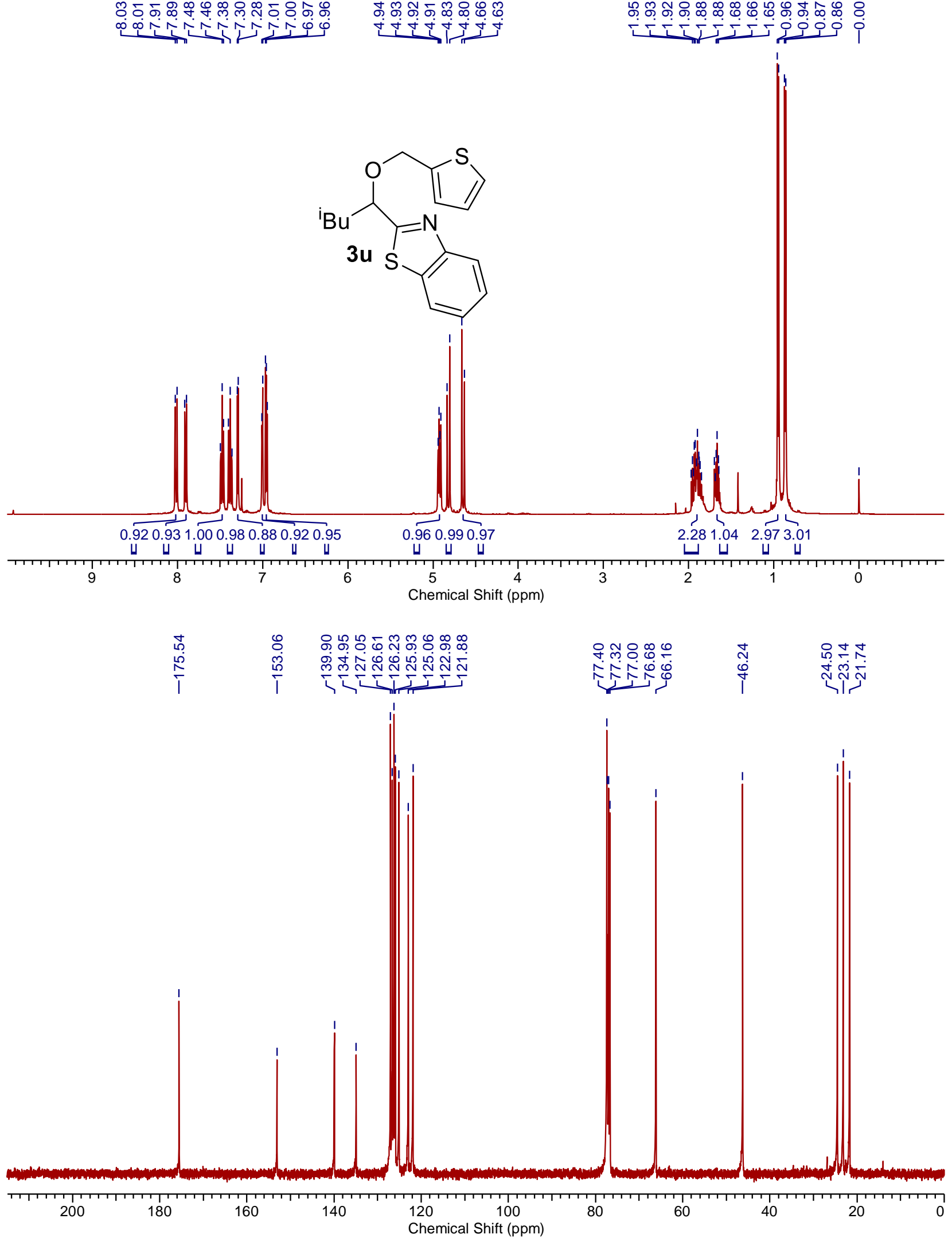

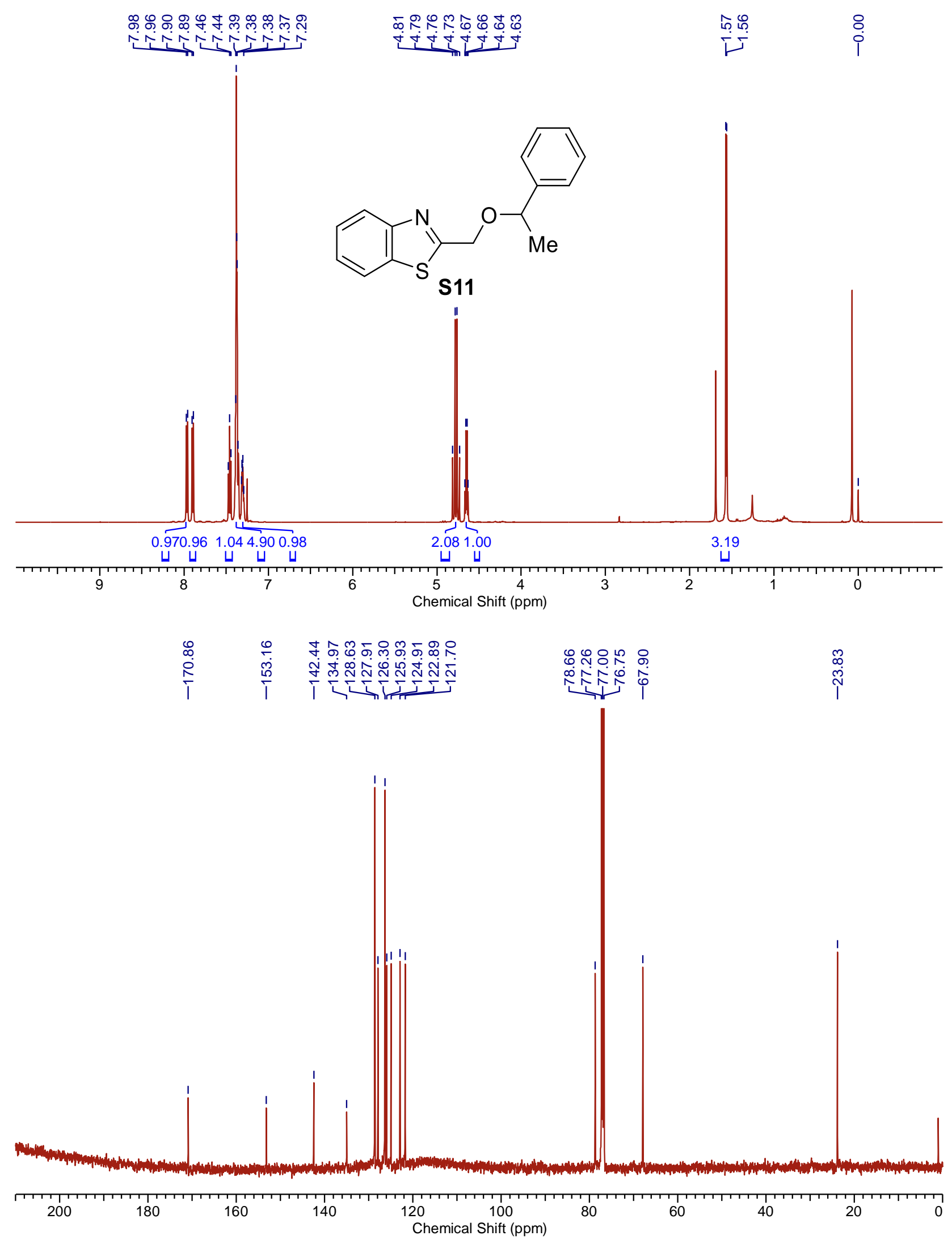


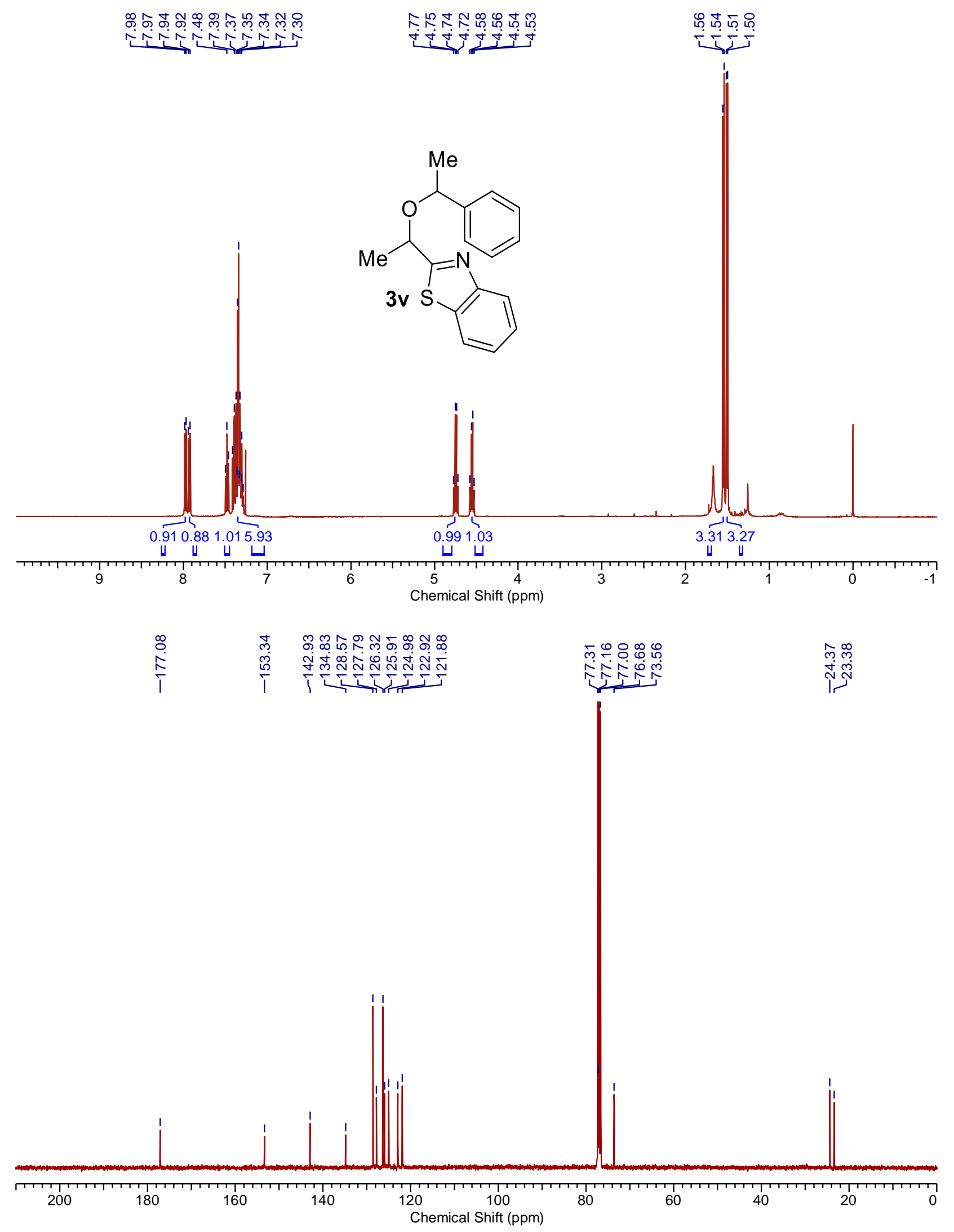




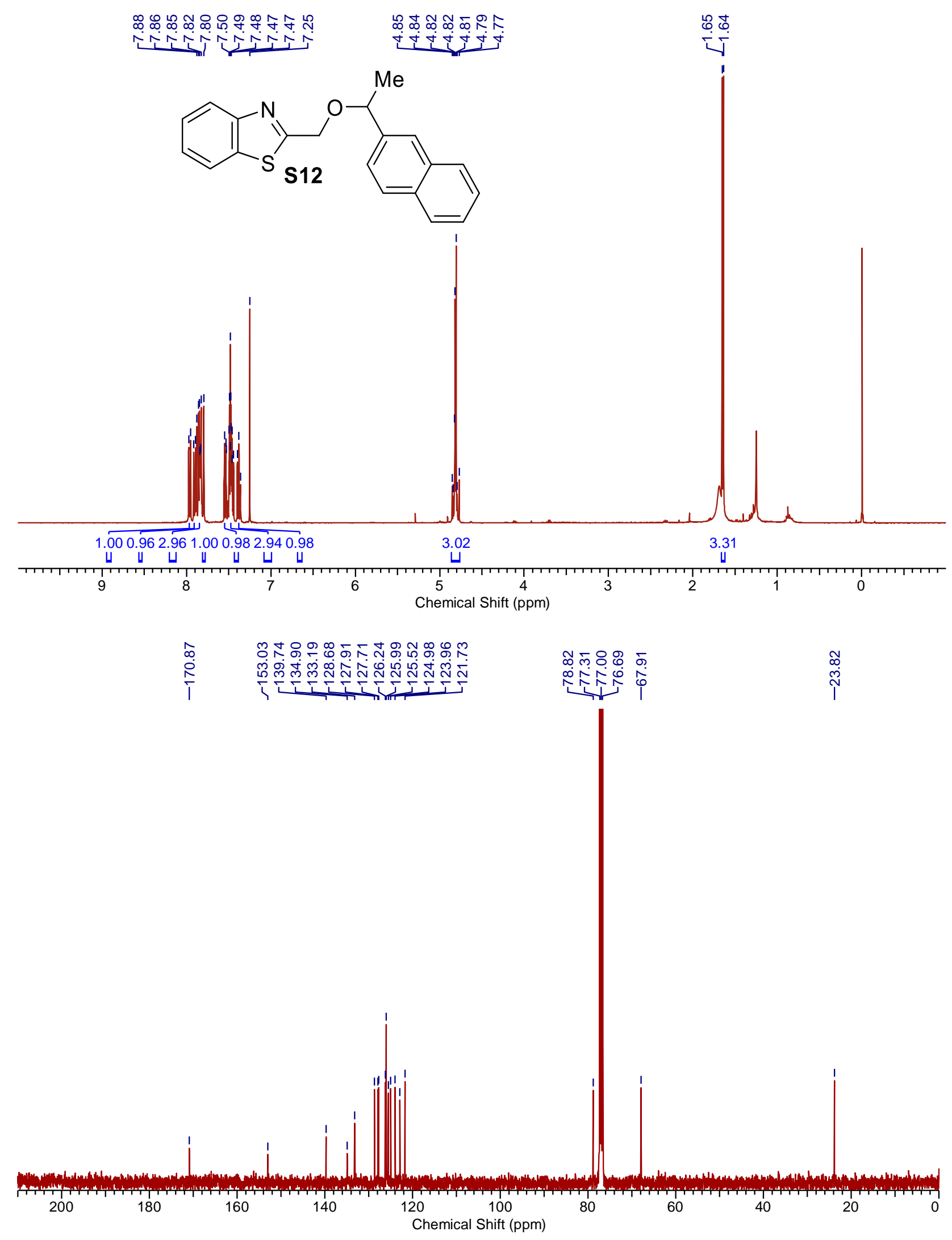




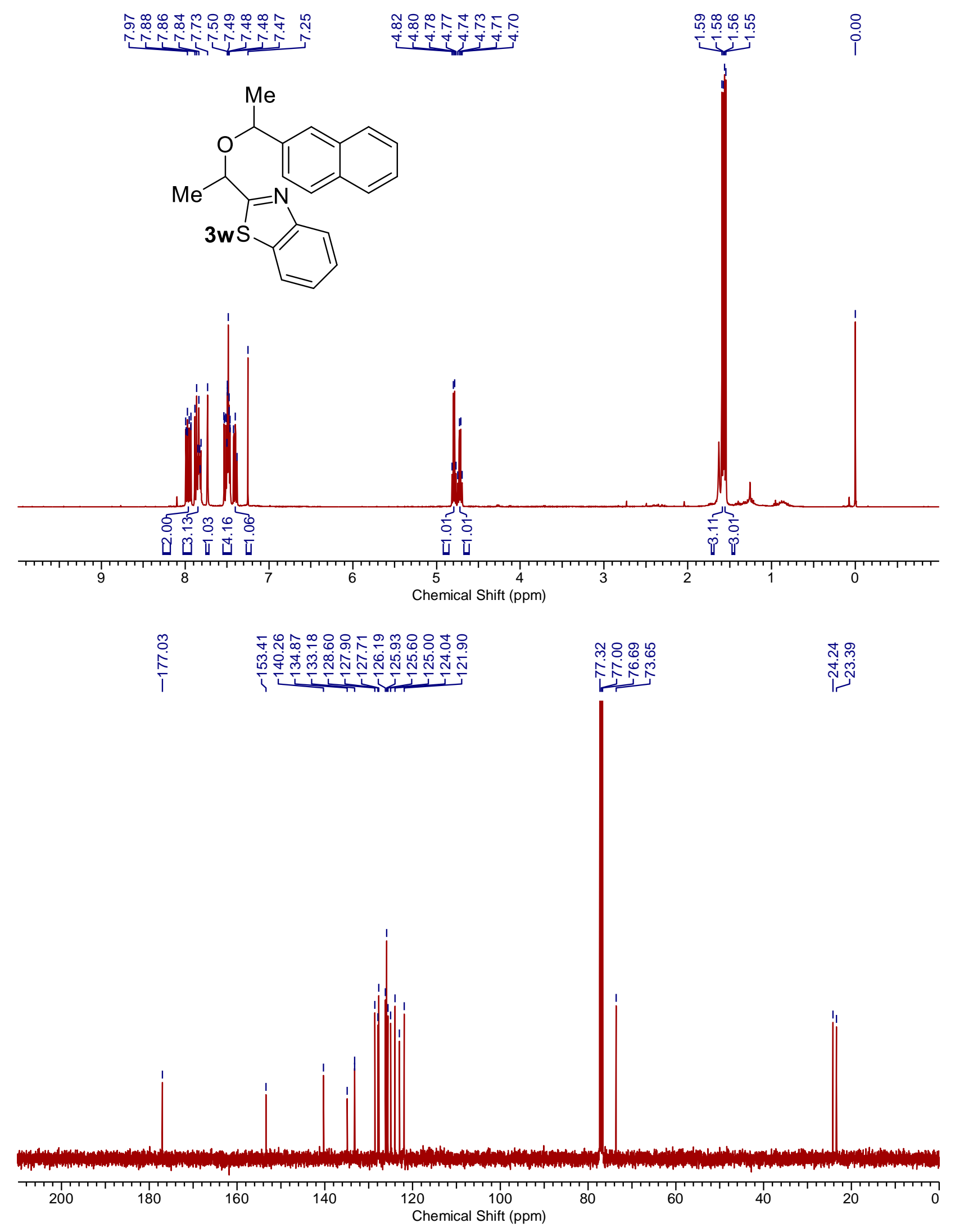



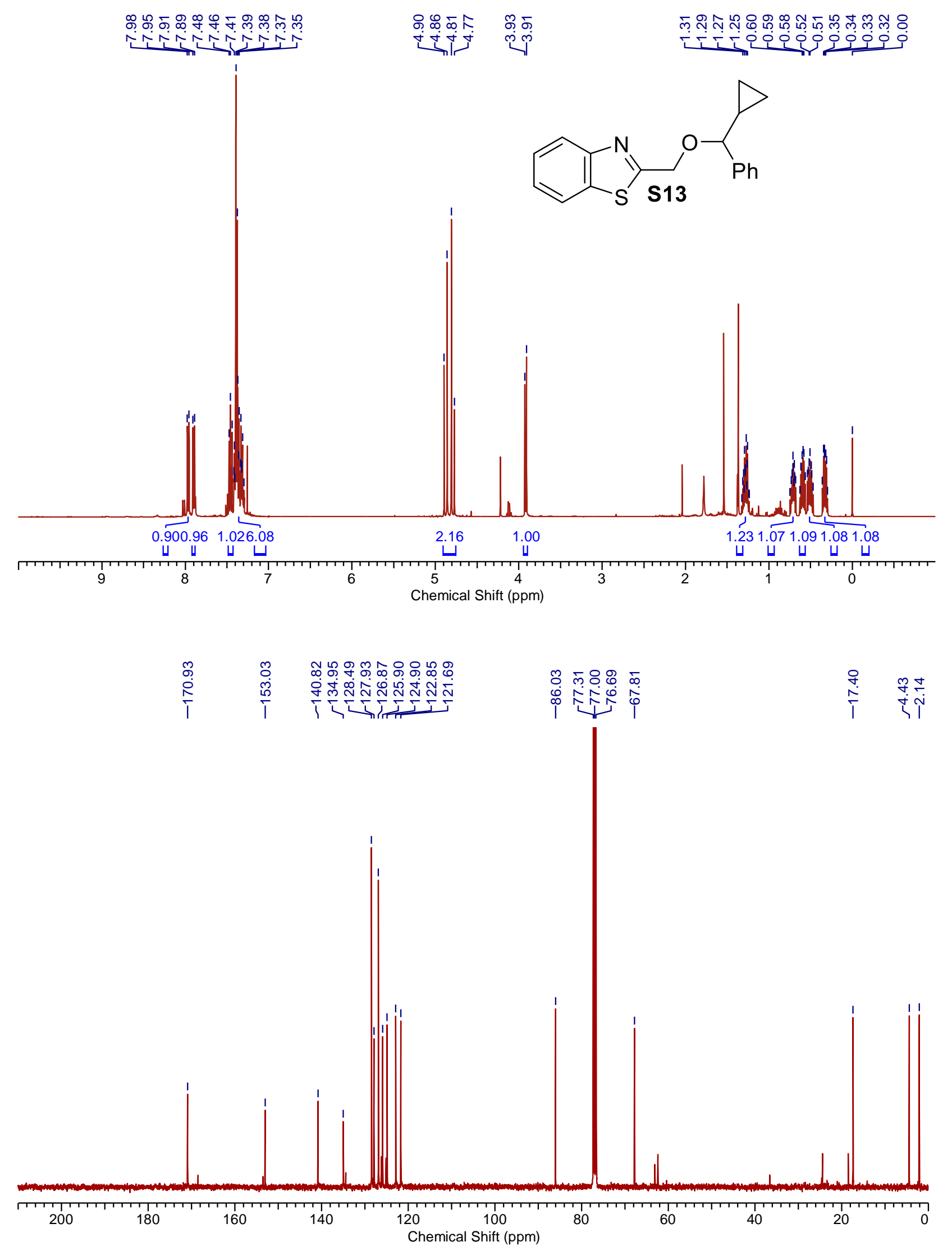


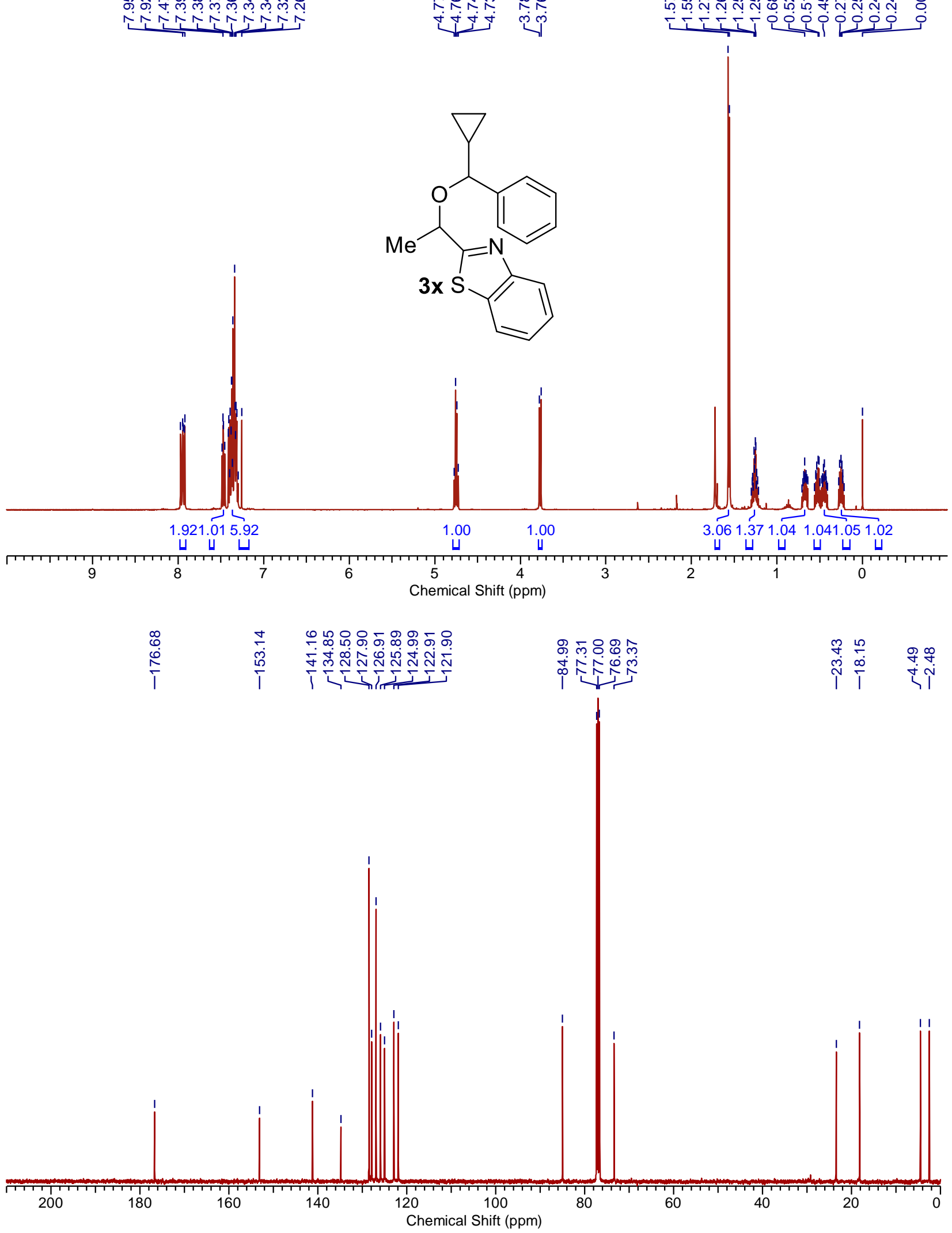



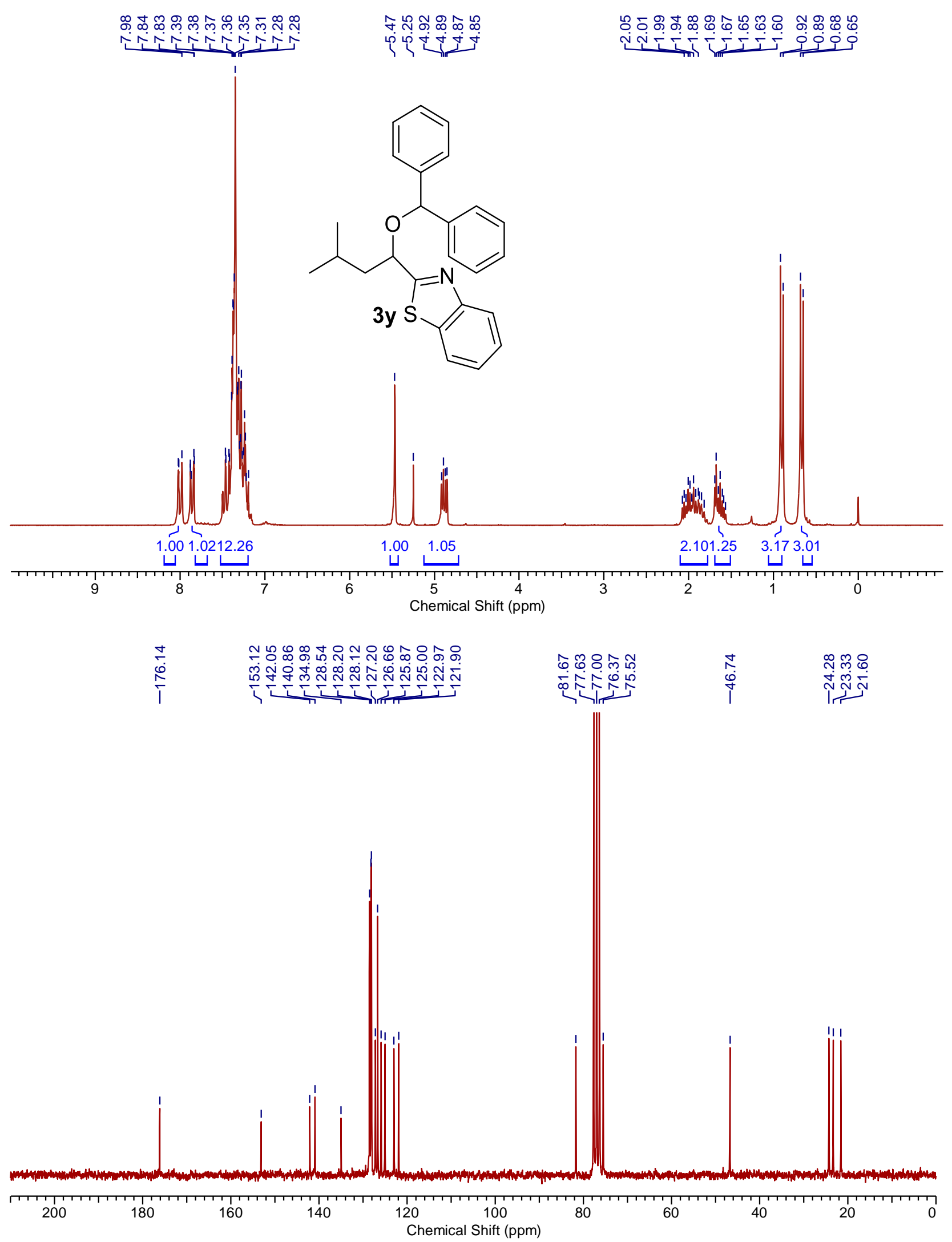

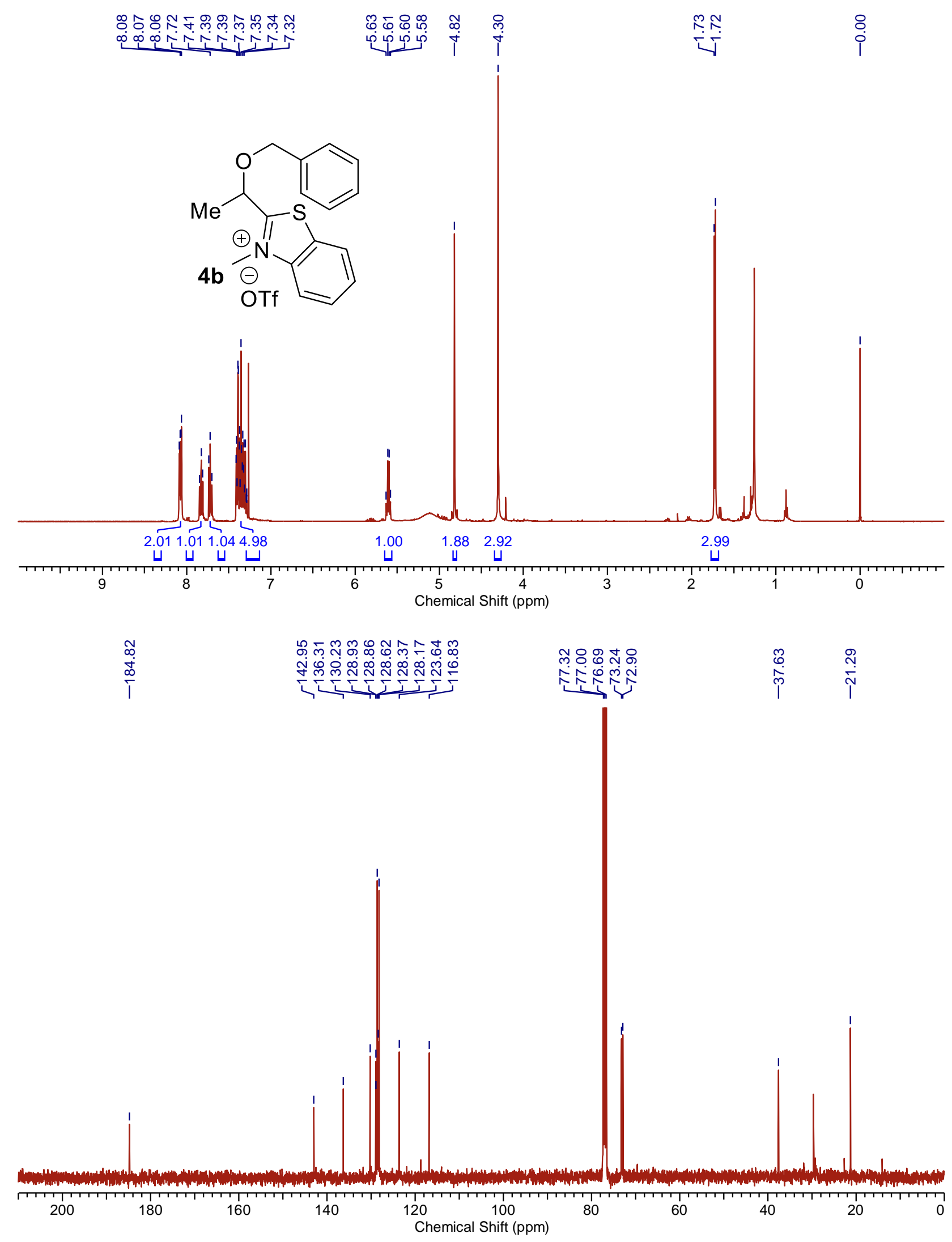

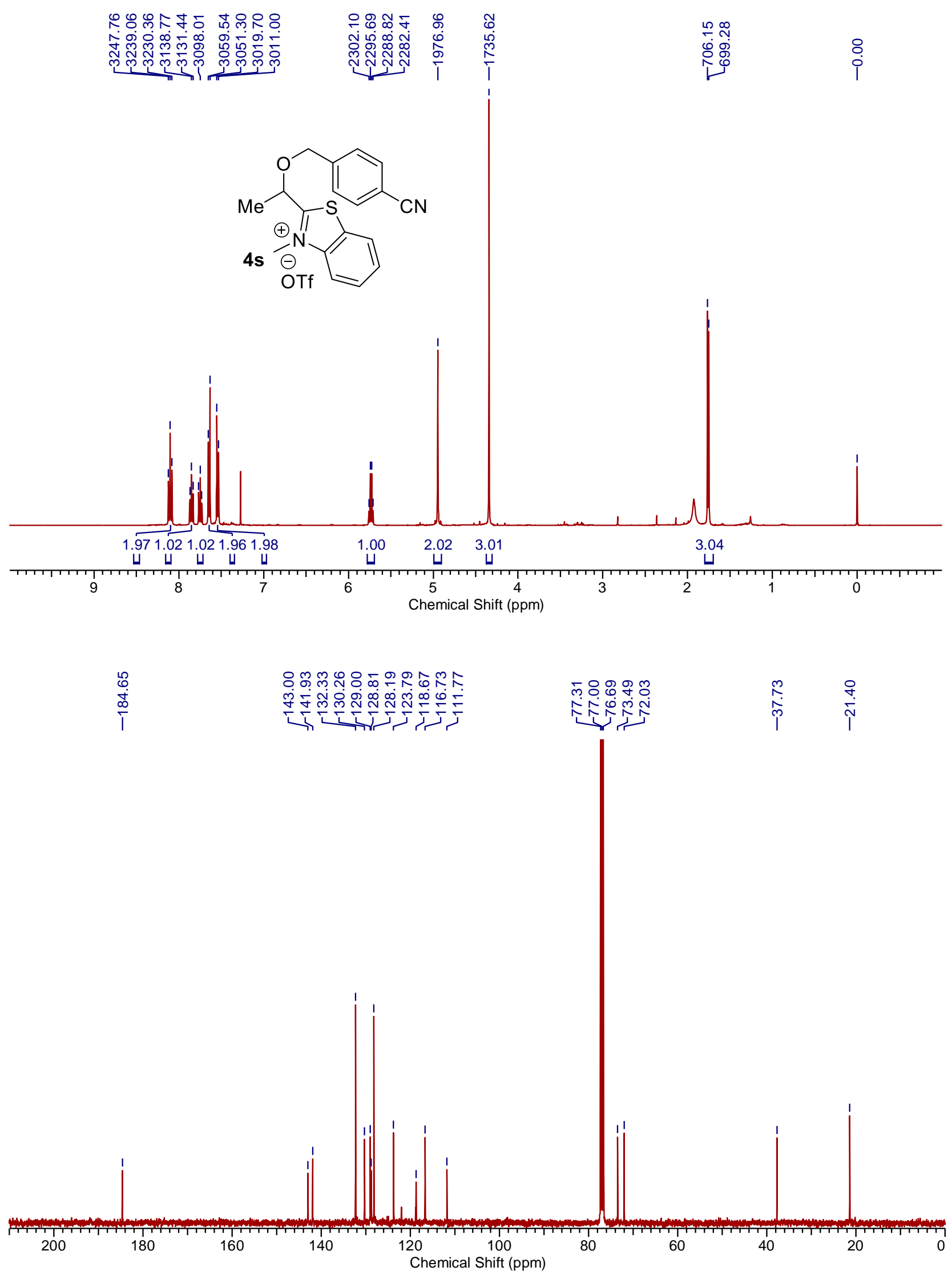

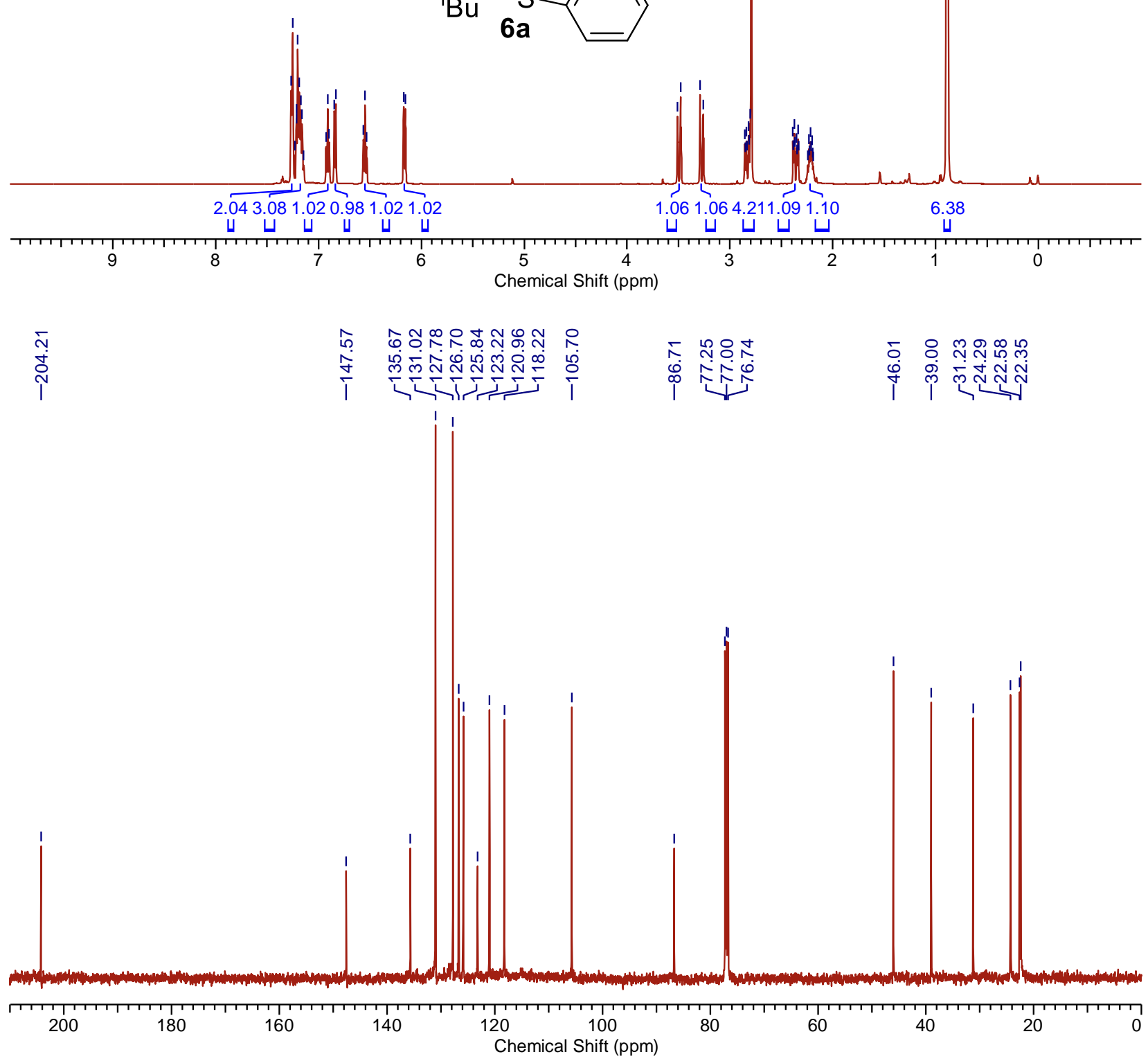

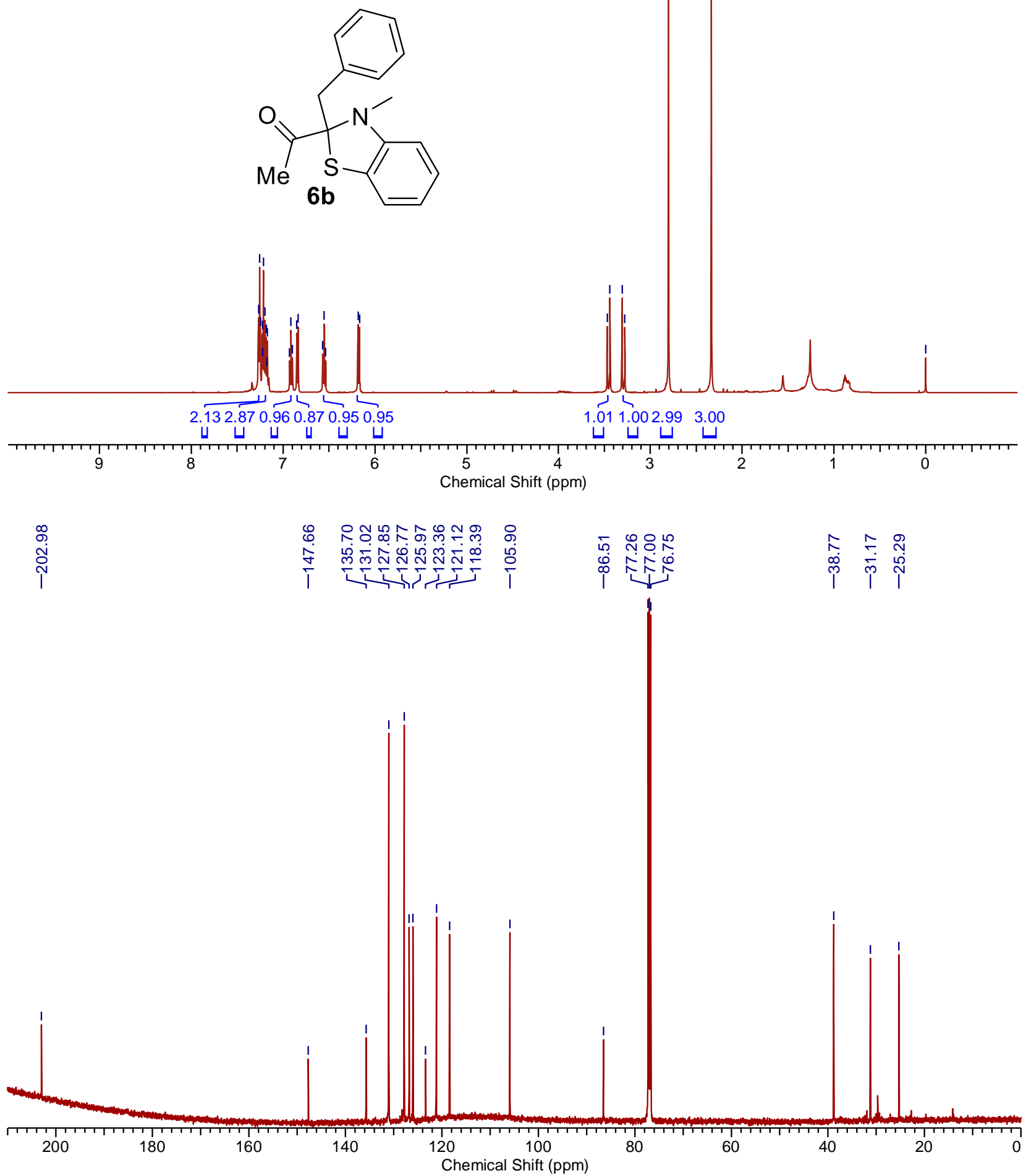

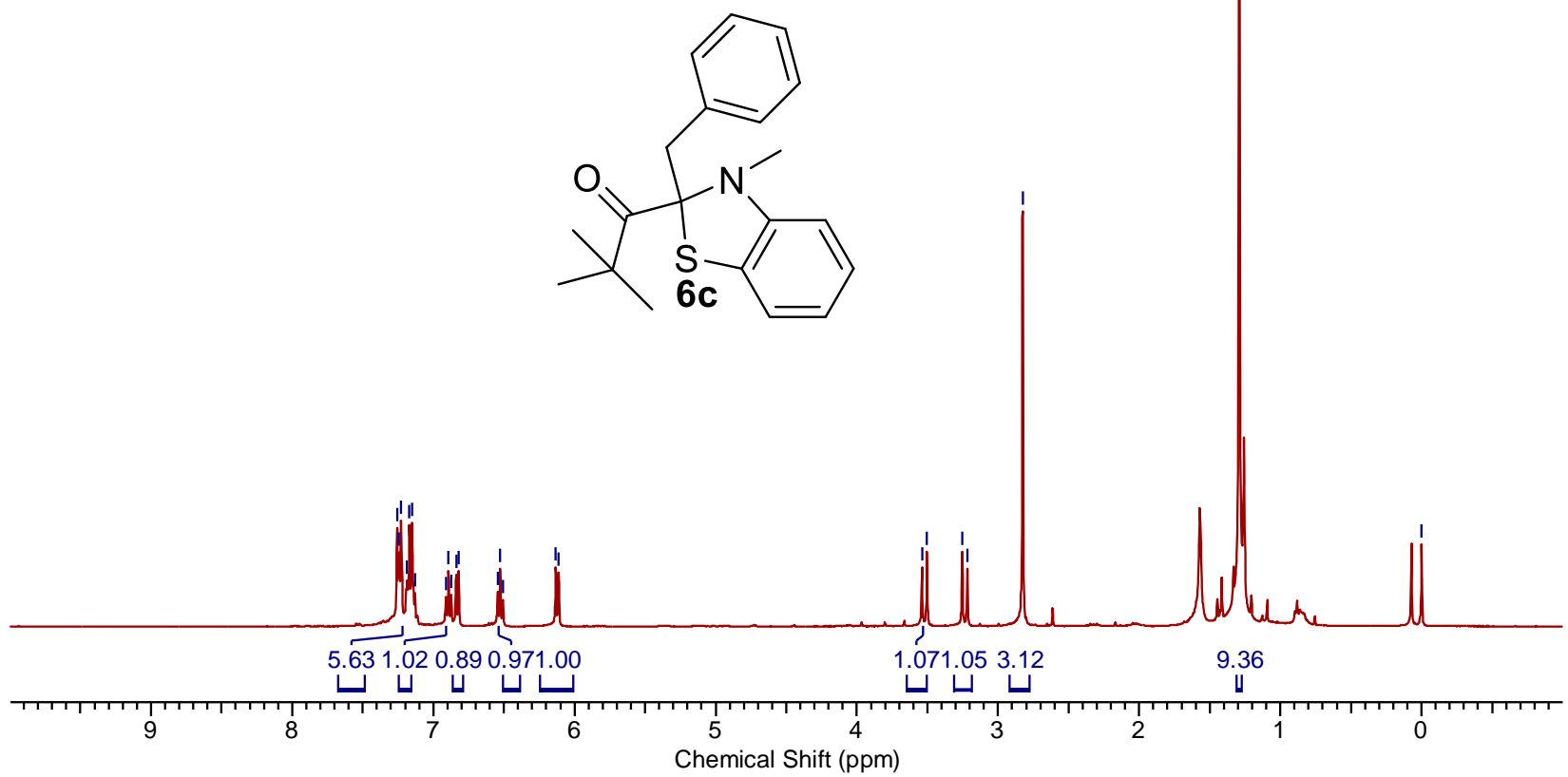

ষ
$\stackrel{N}{1}$
$\stackrel{i}{1}$

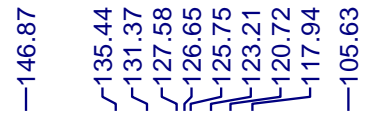

क् ल용

i 余余

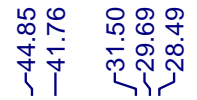

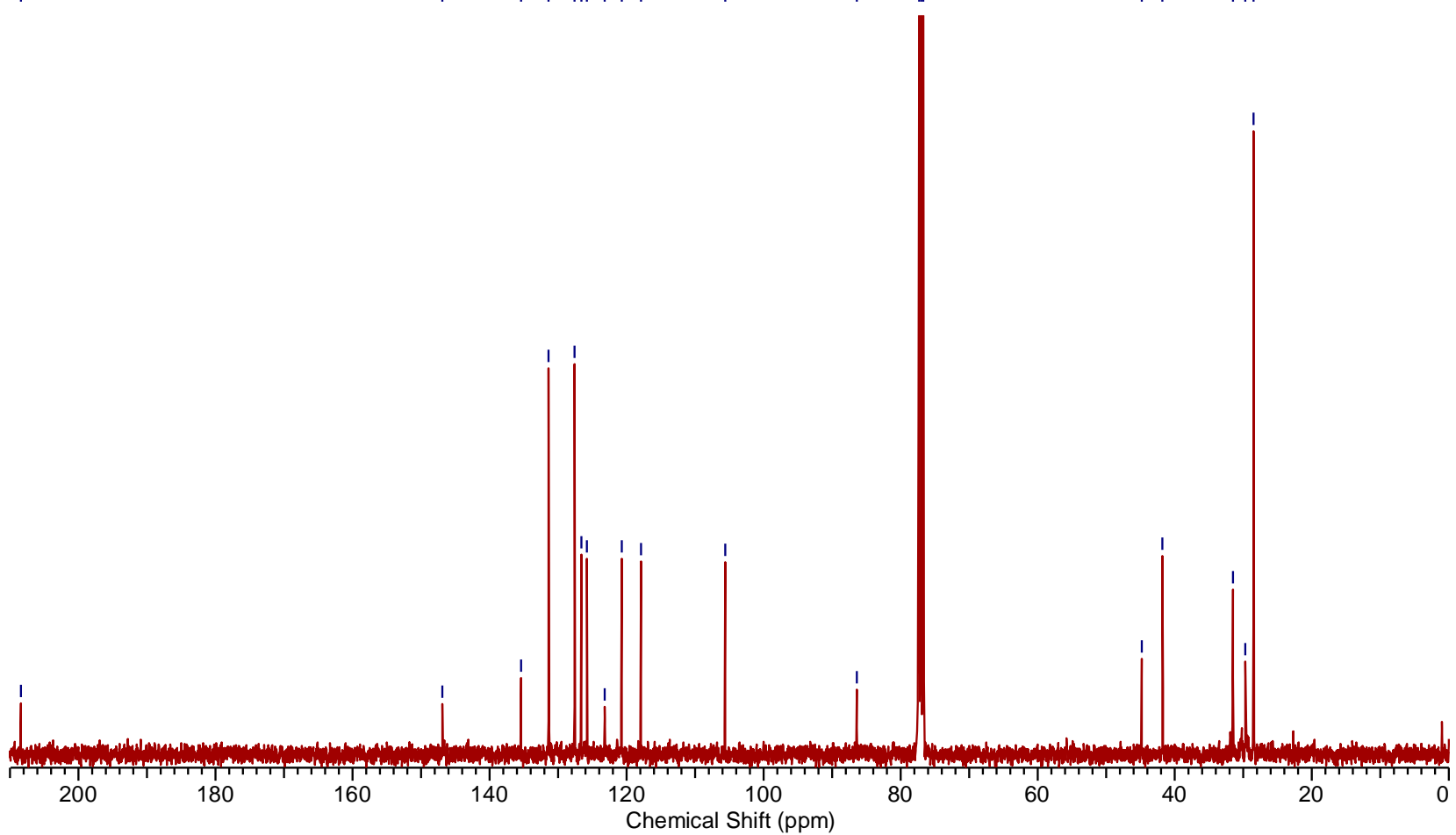



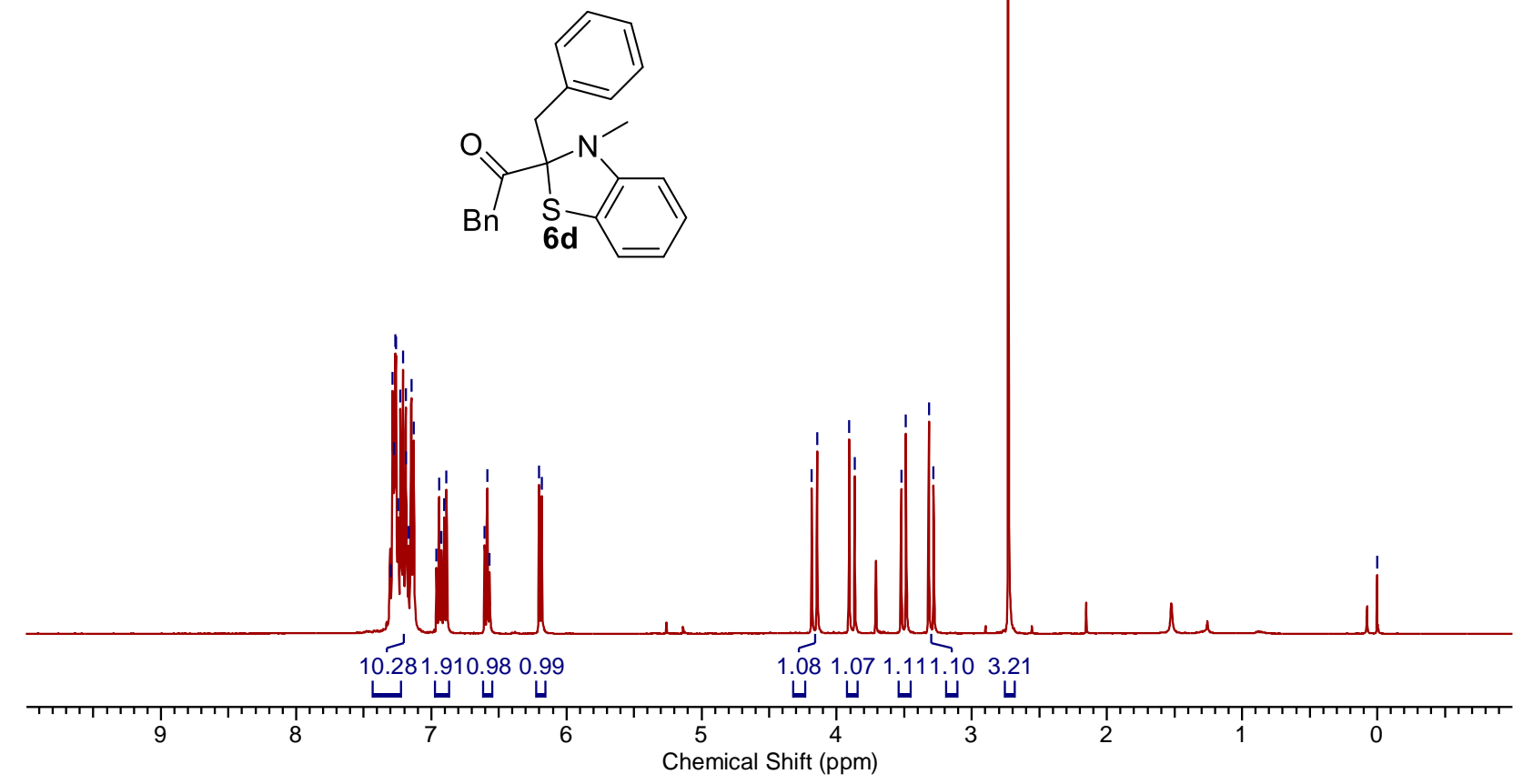

ำ

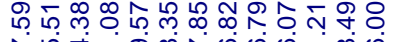

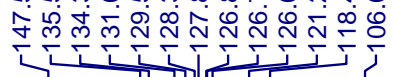

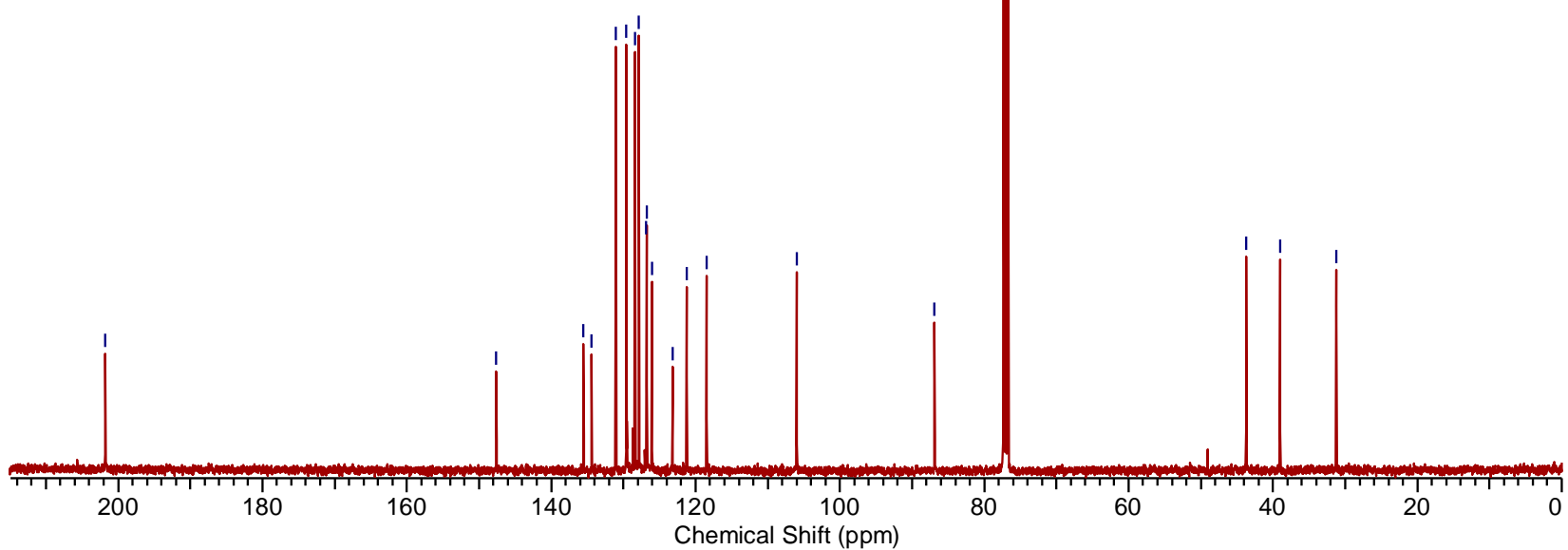



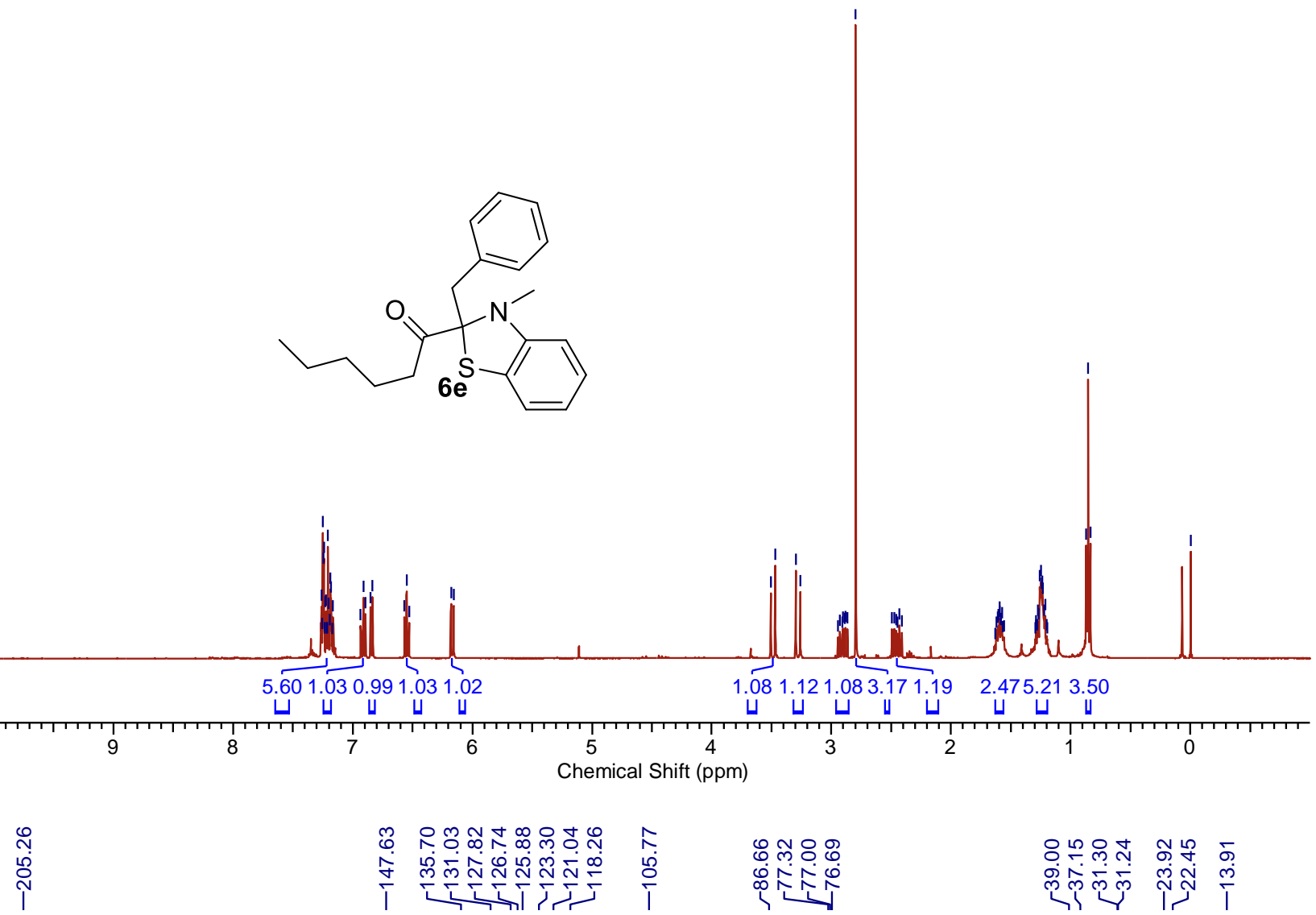

\section{요요}

䟻余

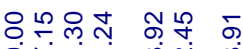

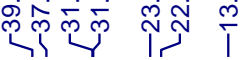

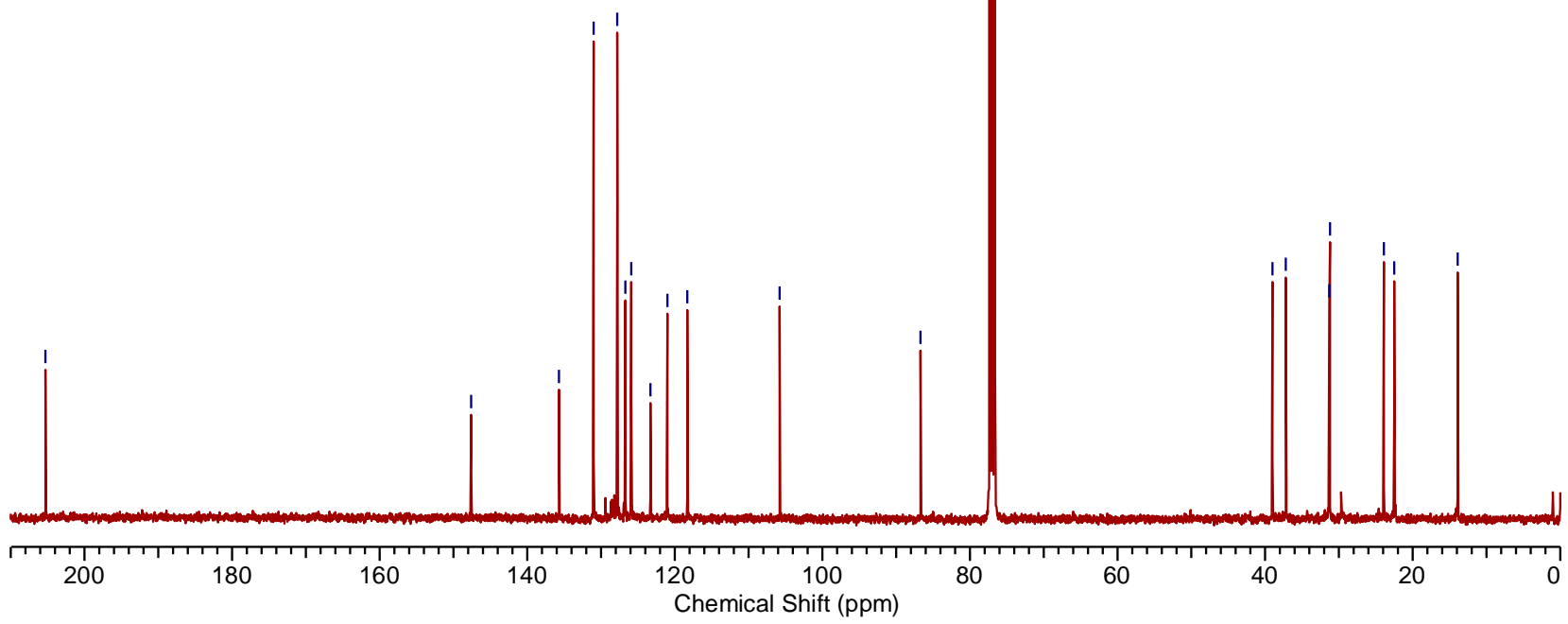



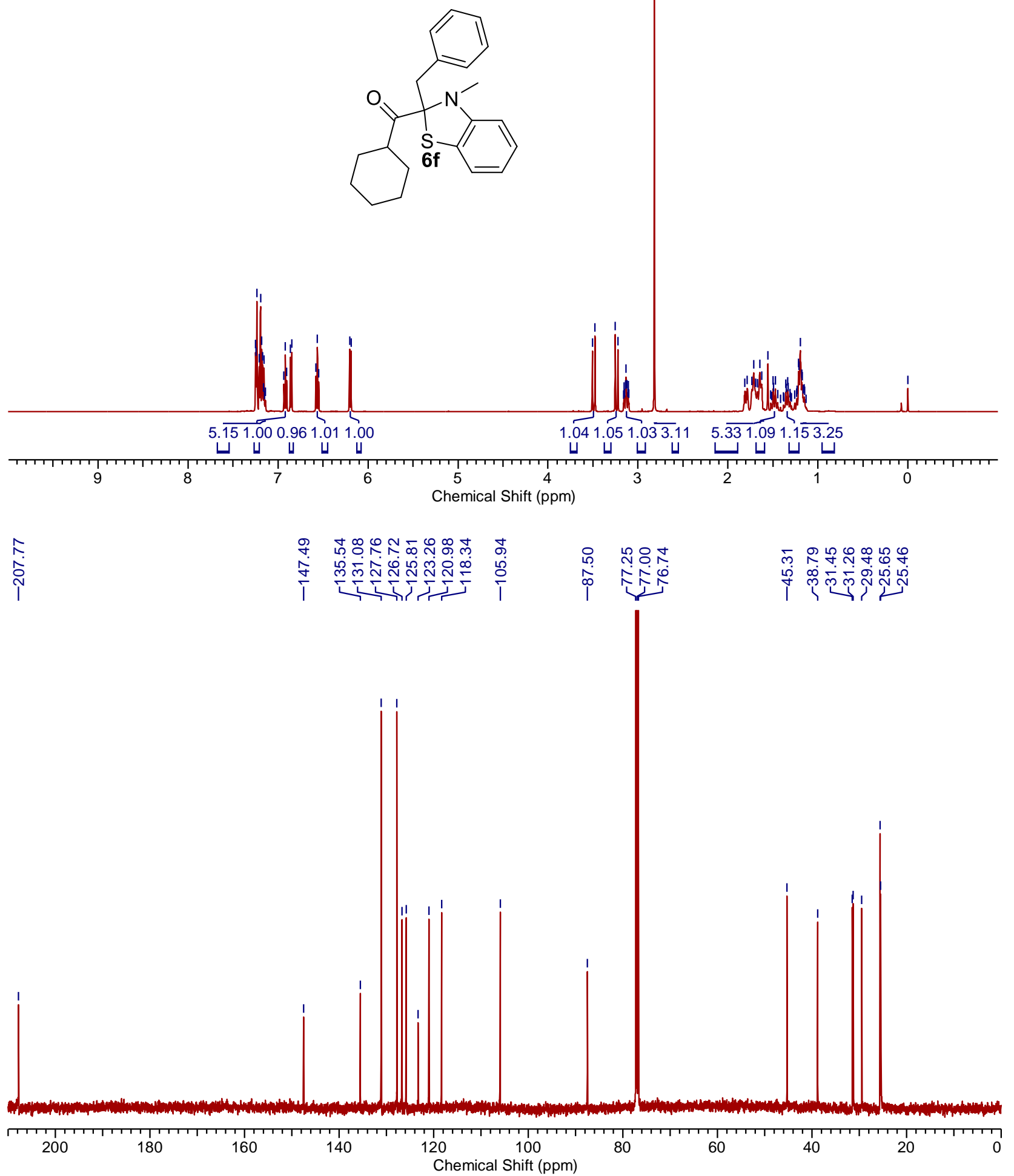

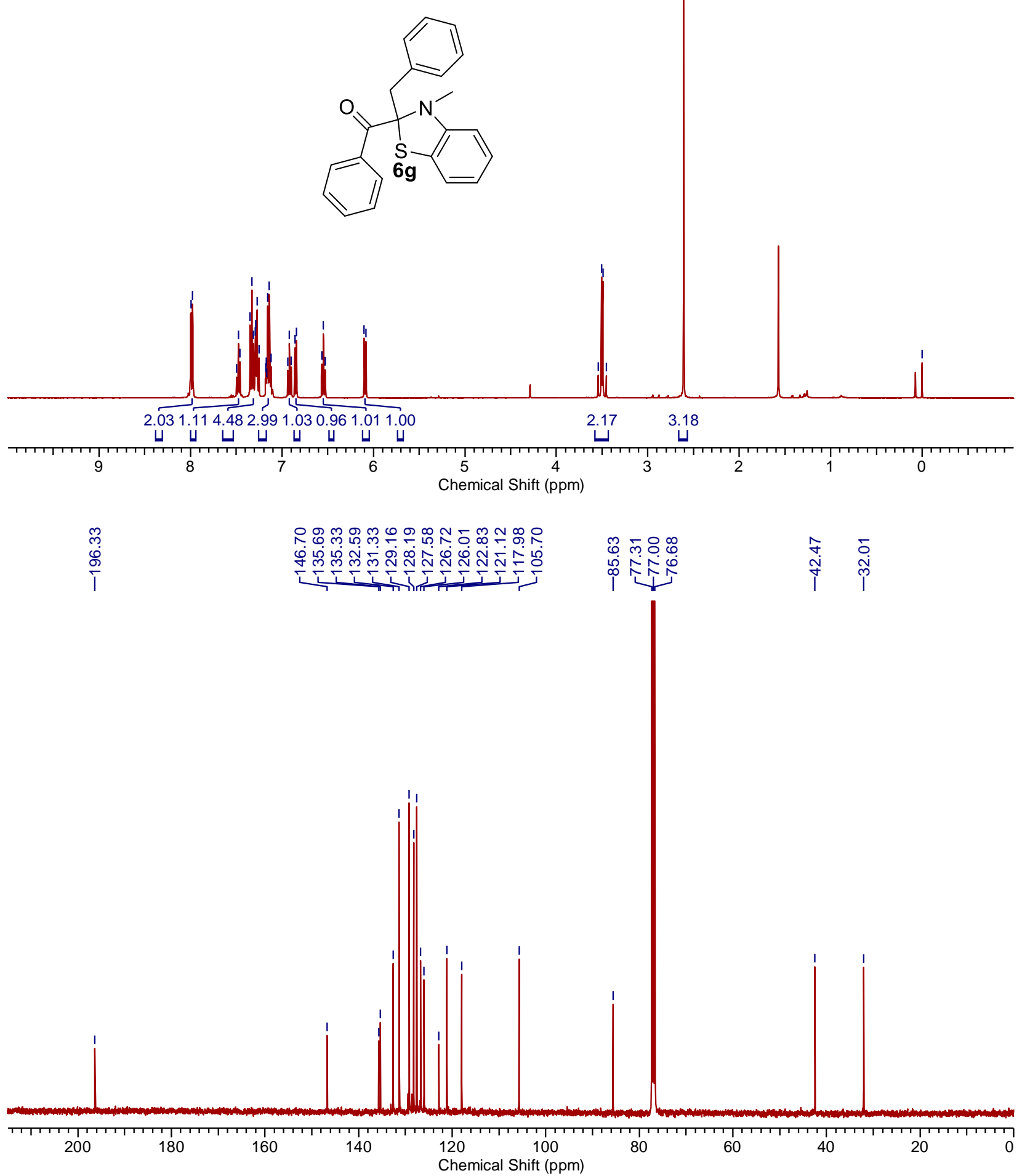

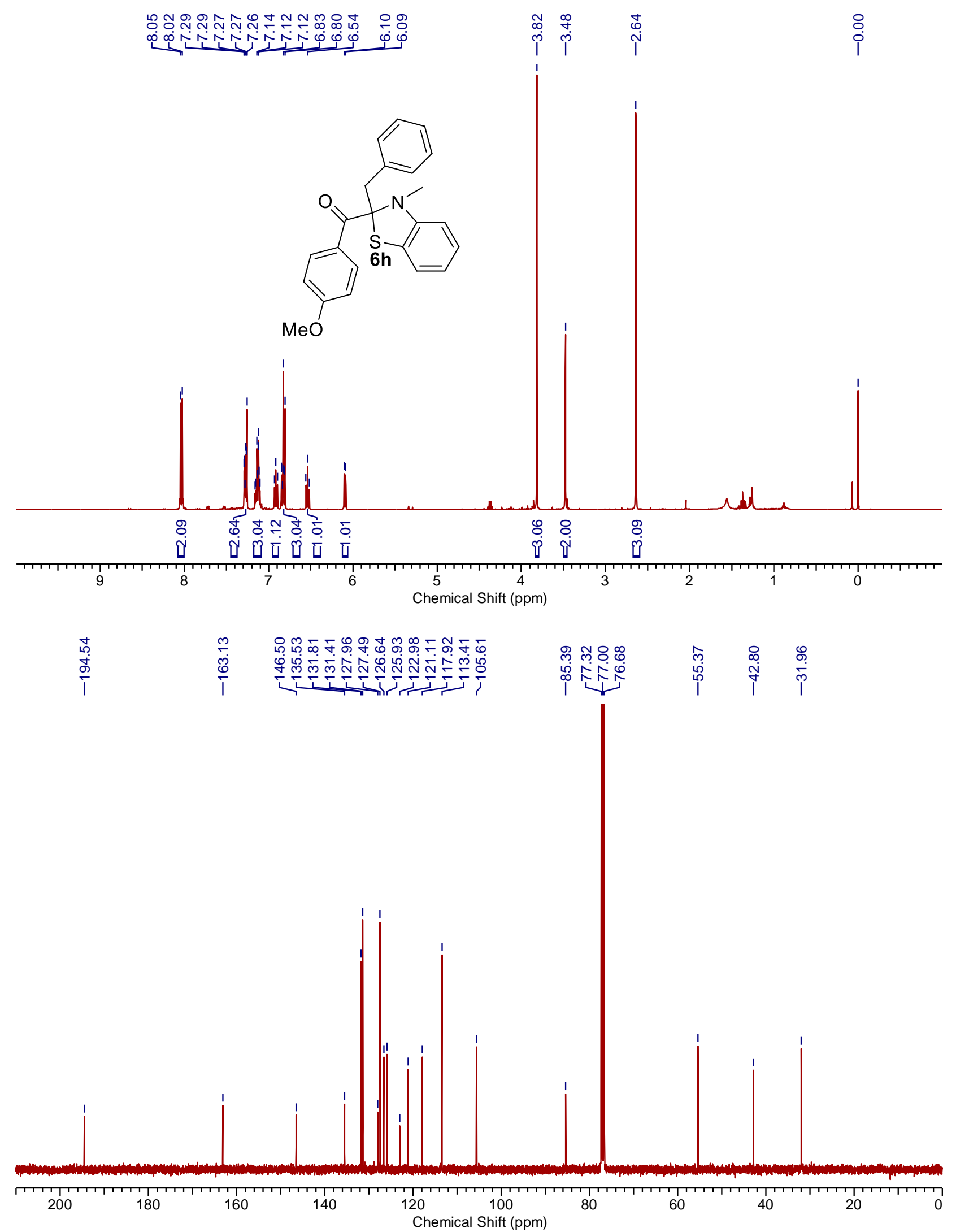


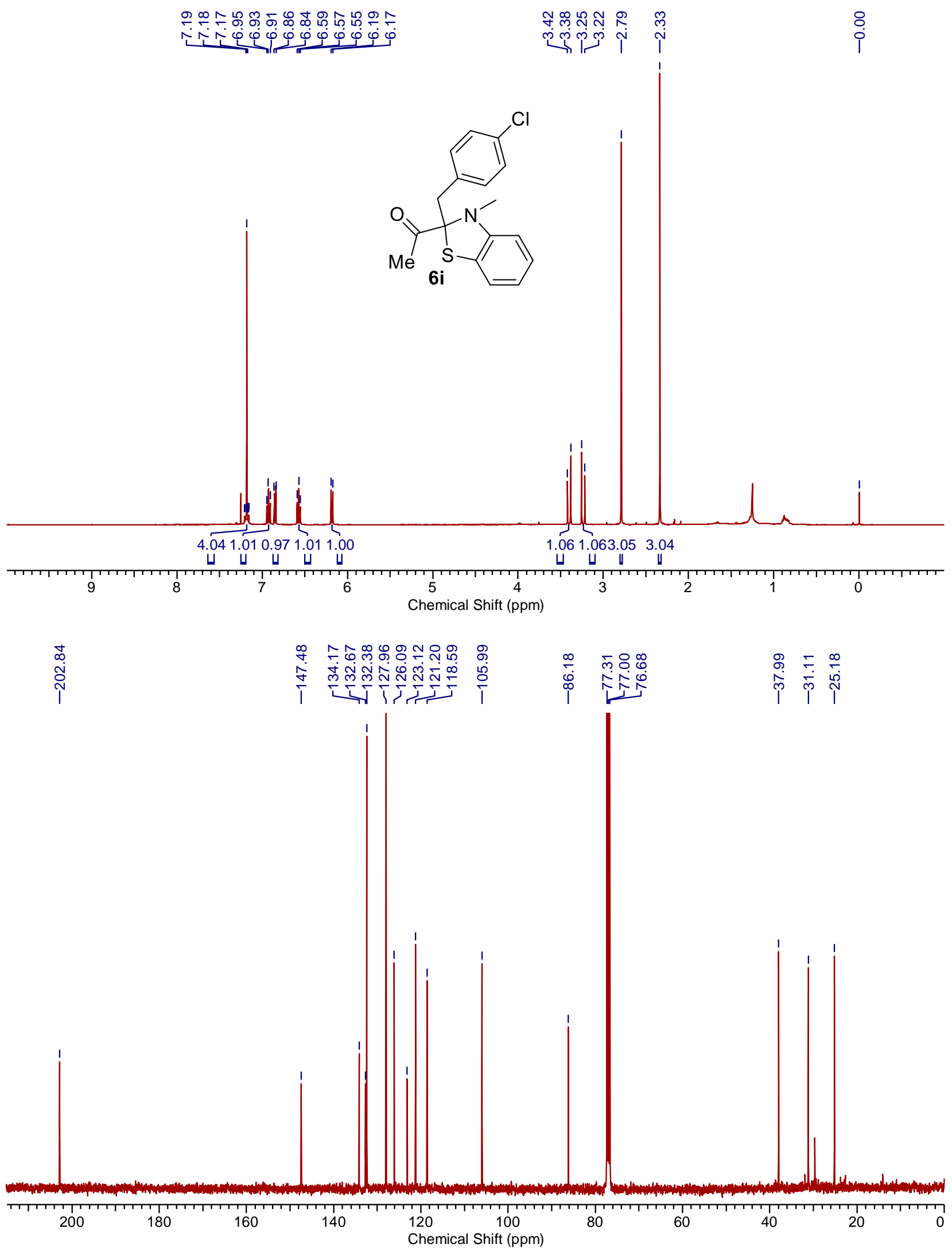




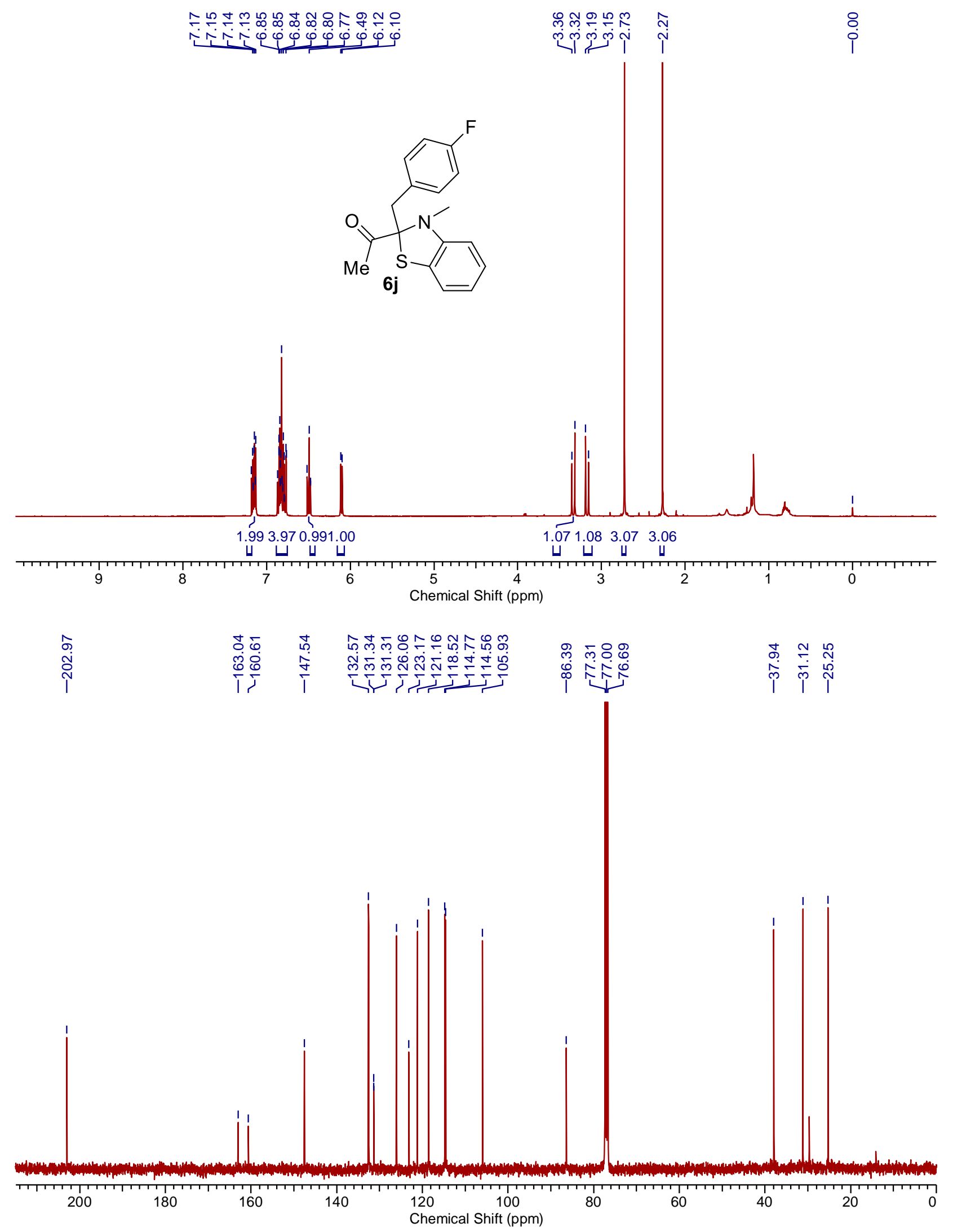



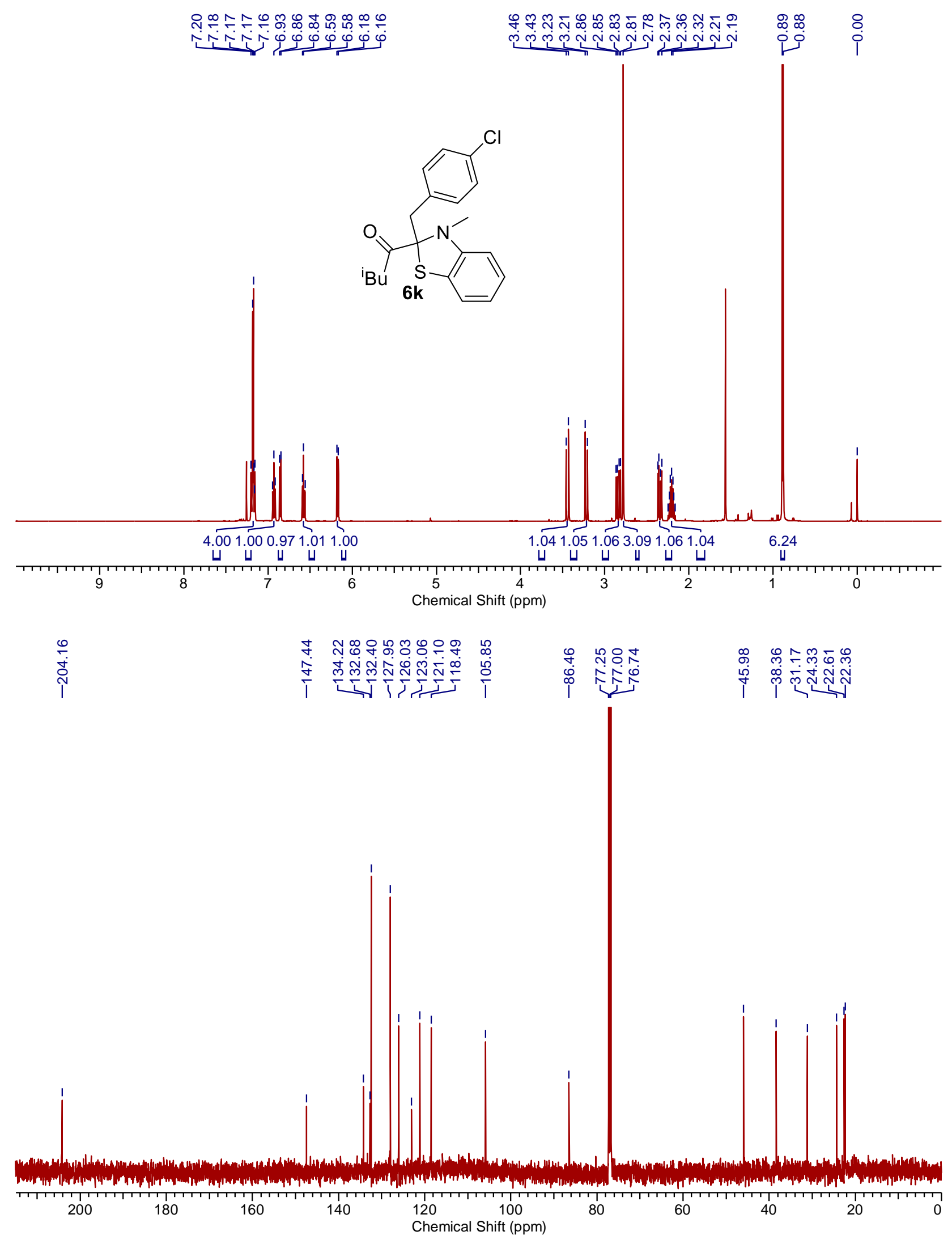

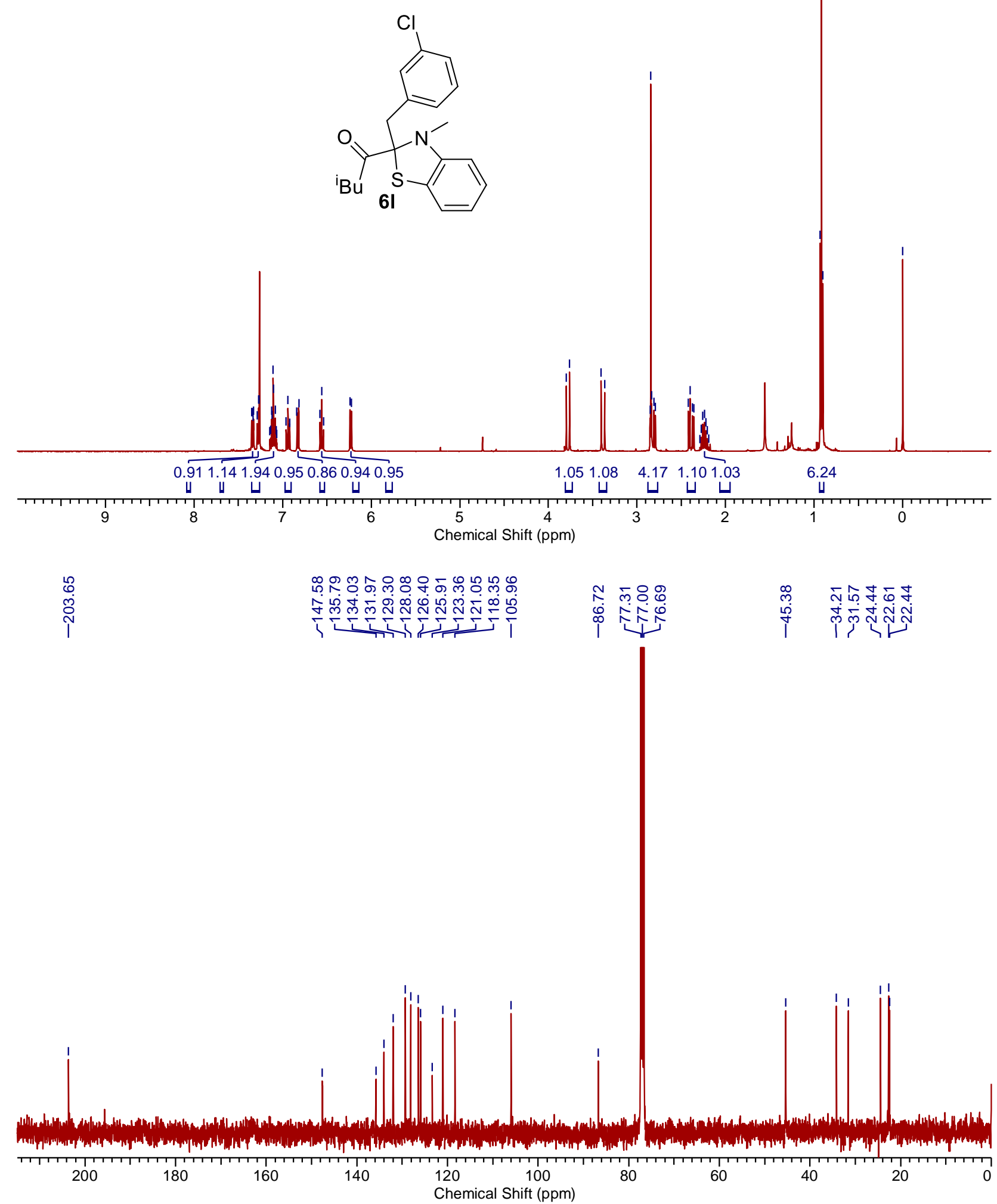

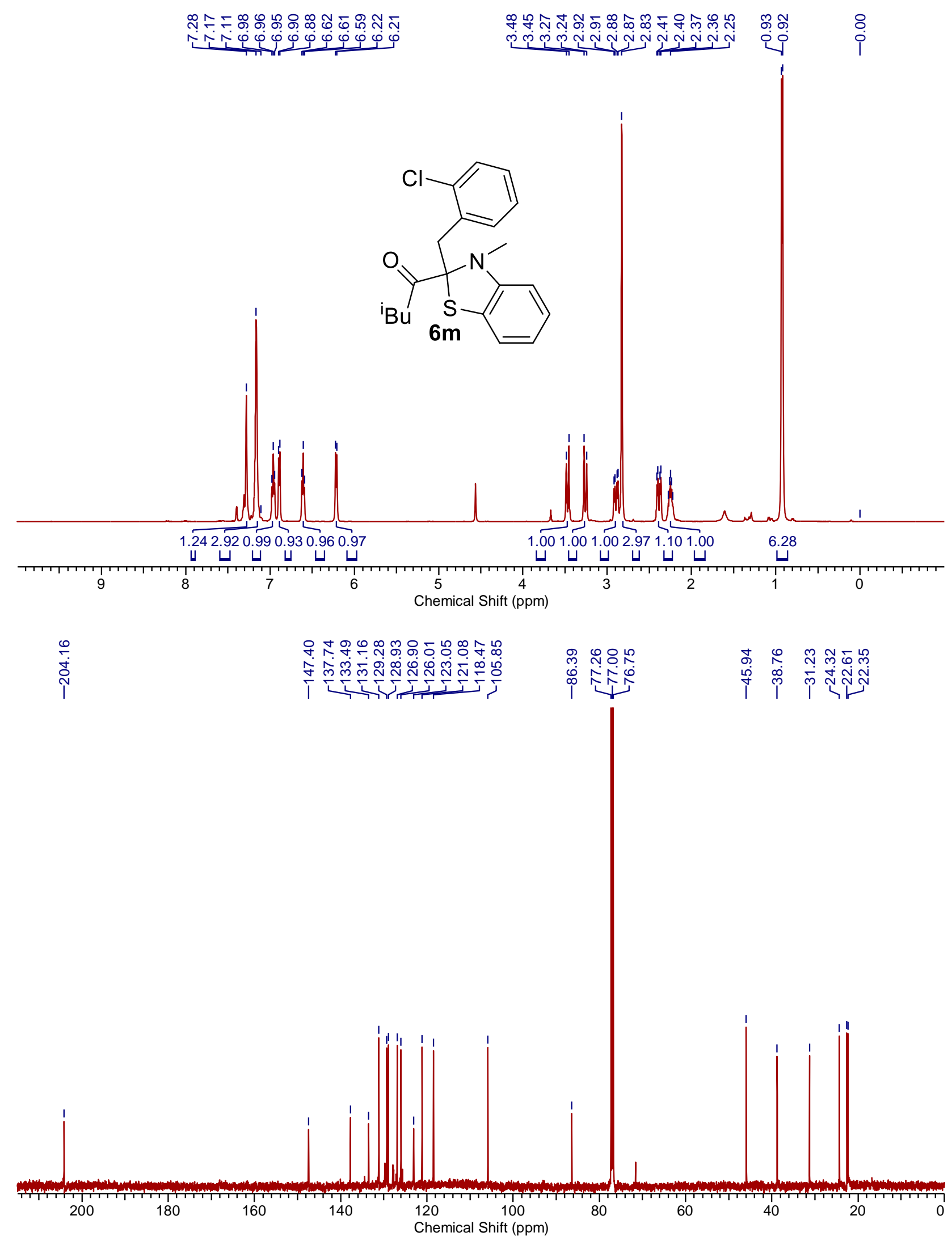


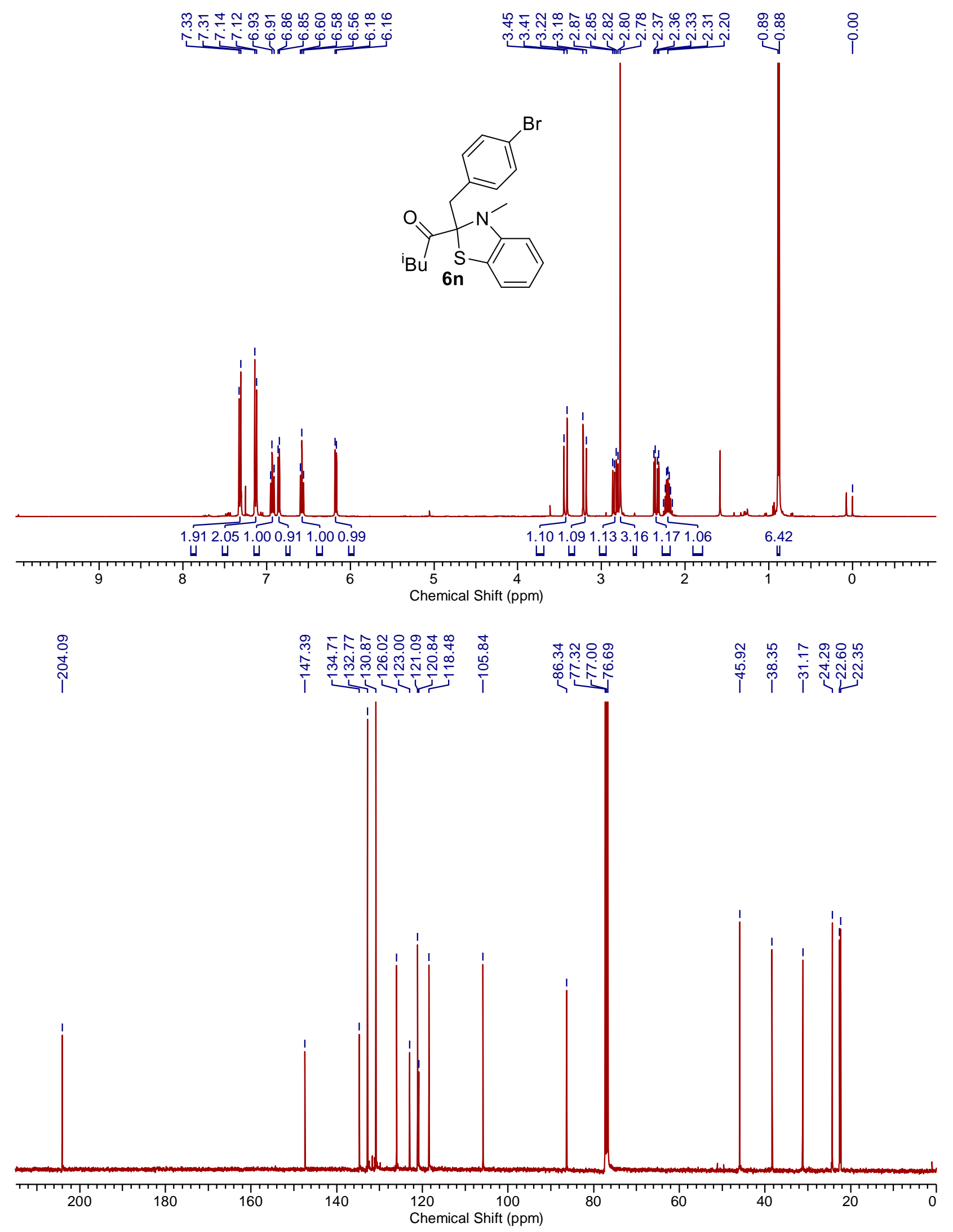




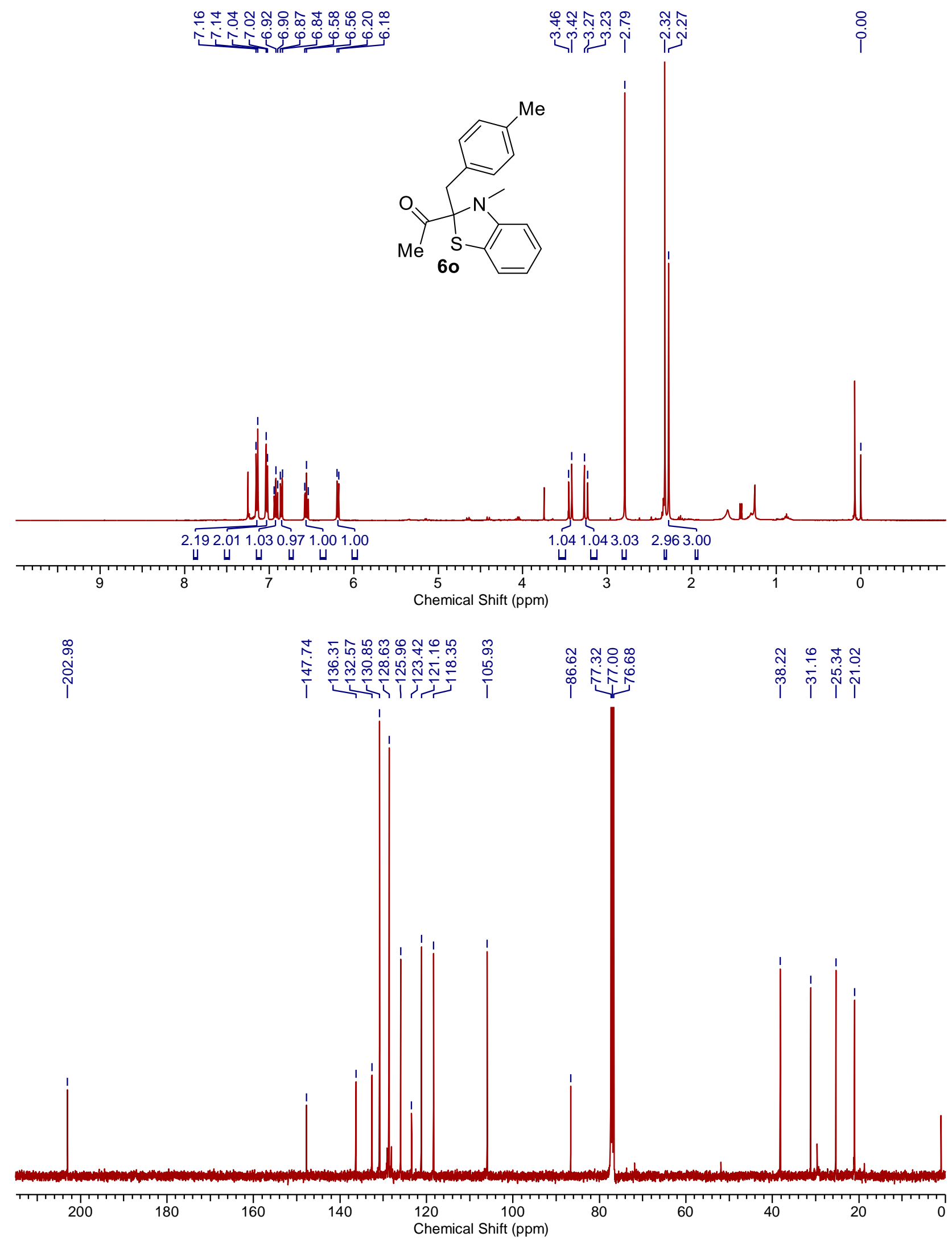



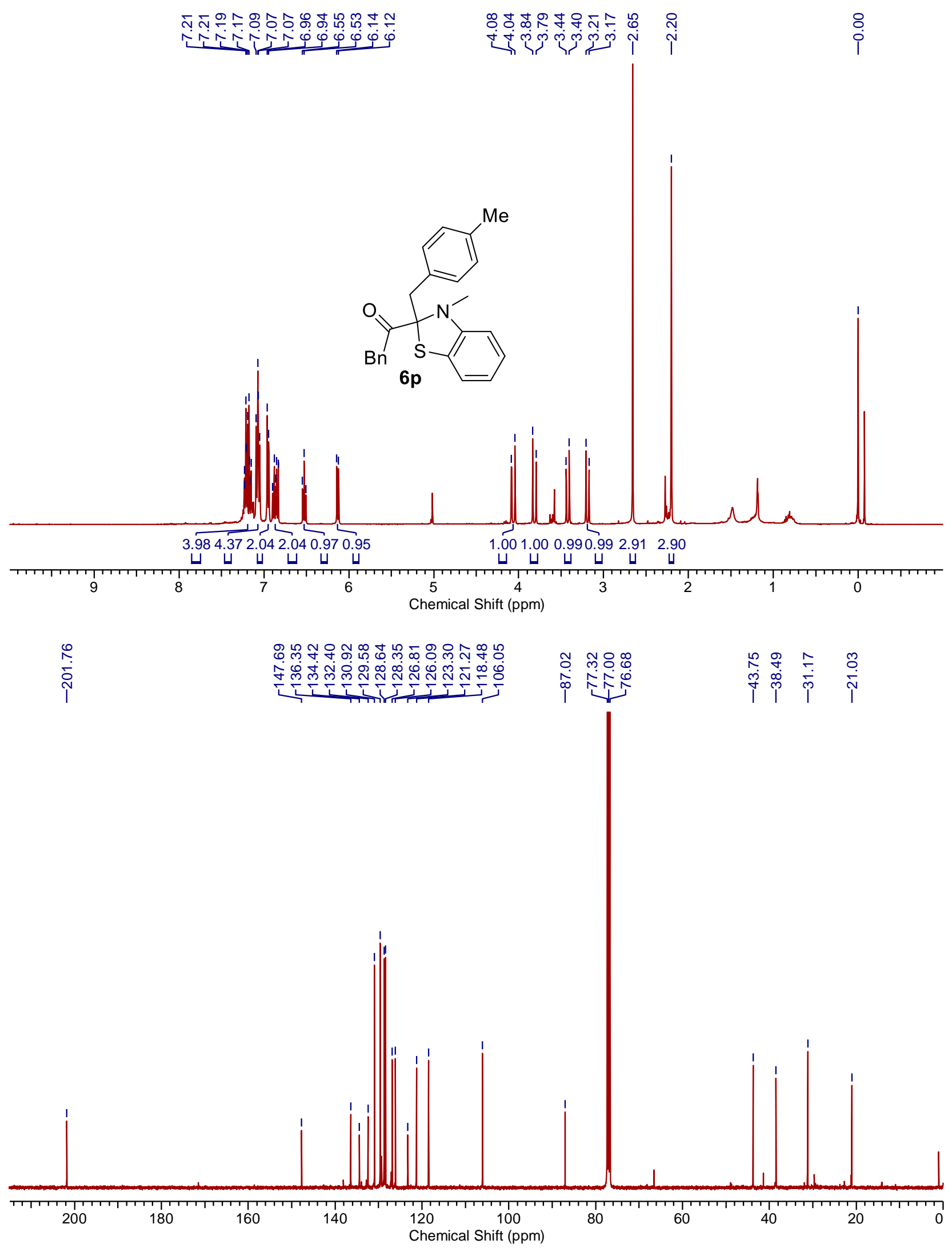

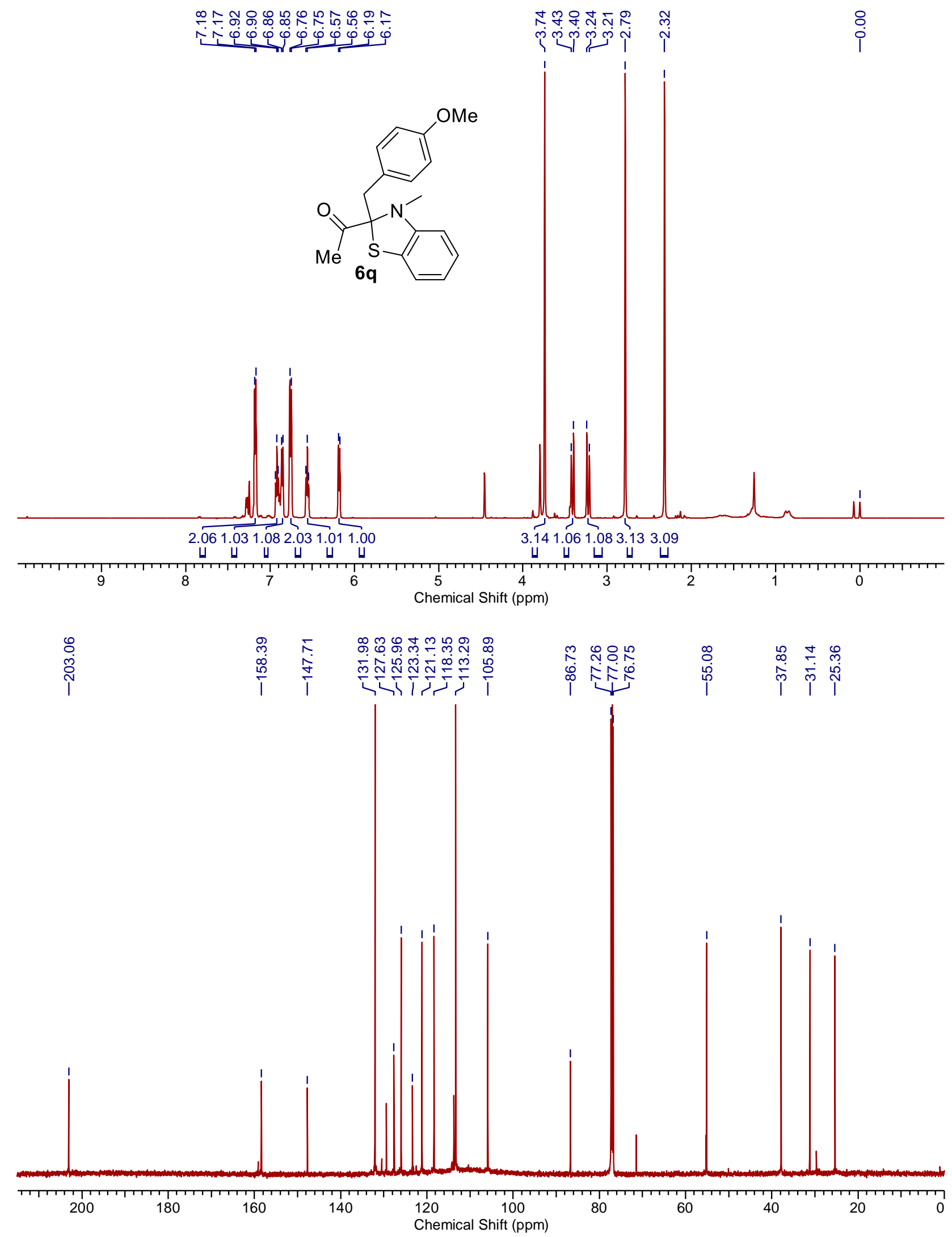


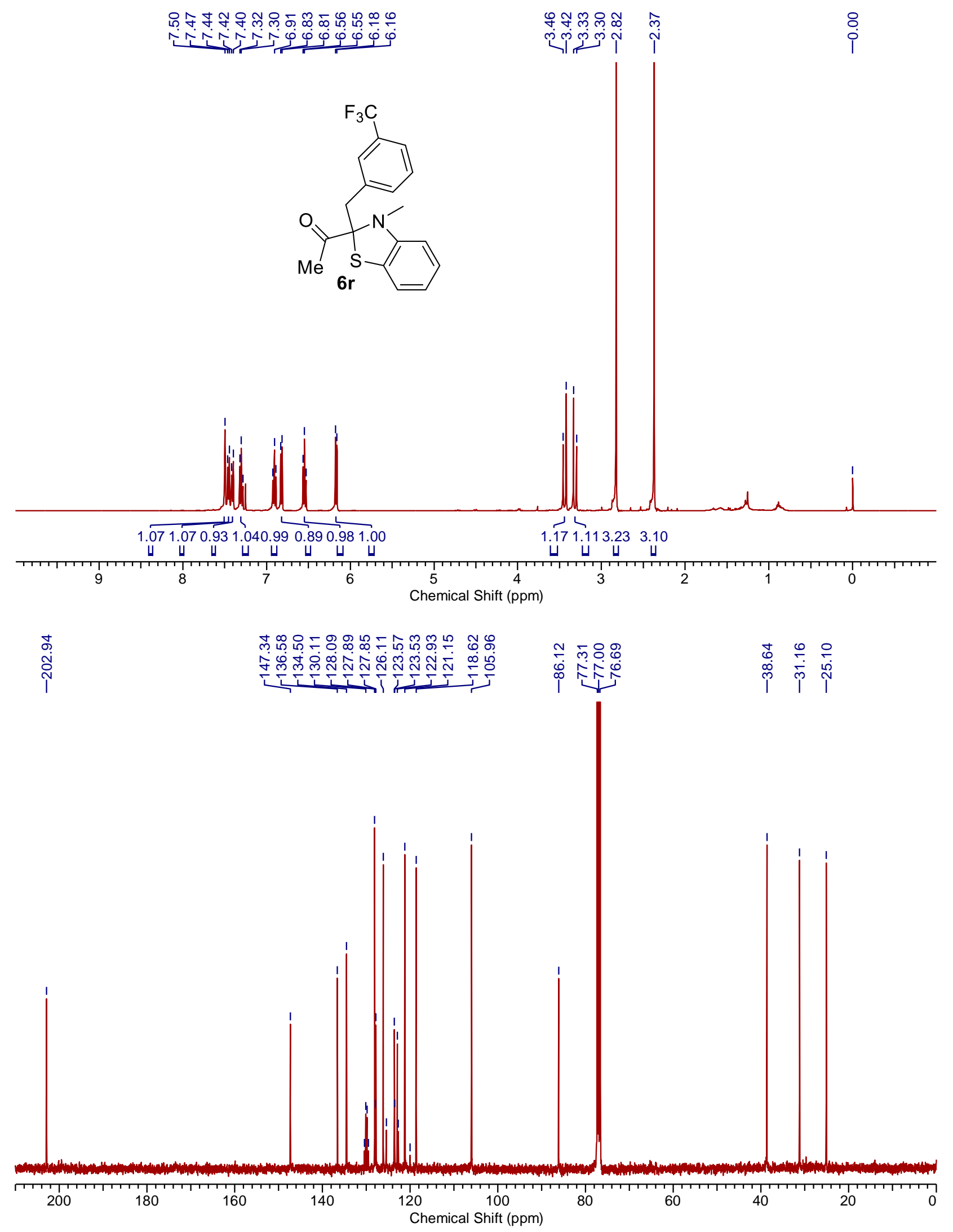




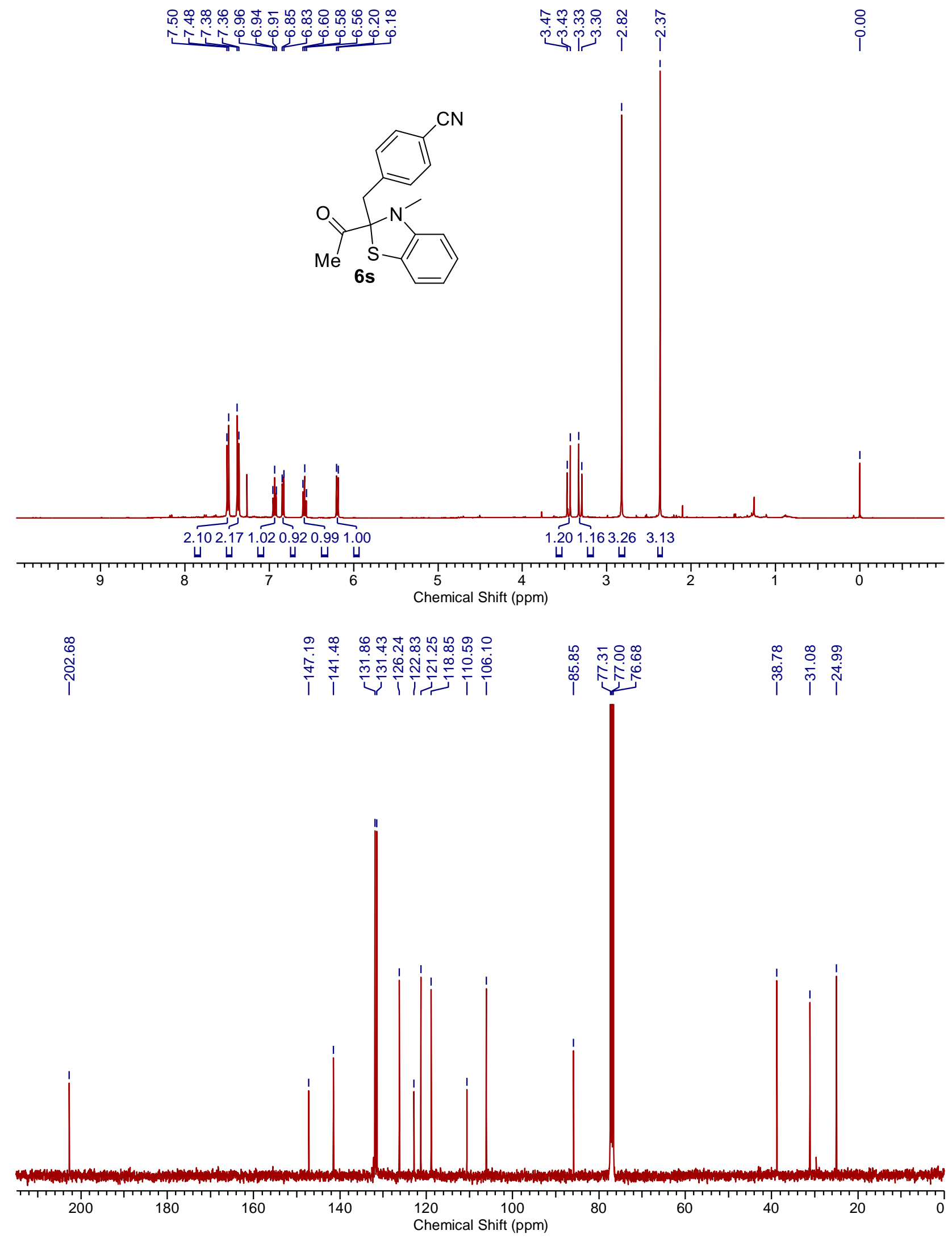




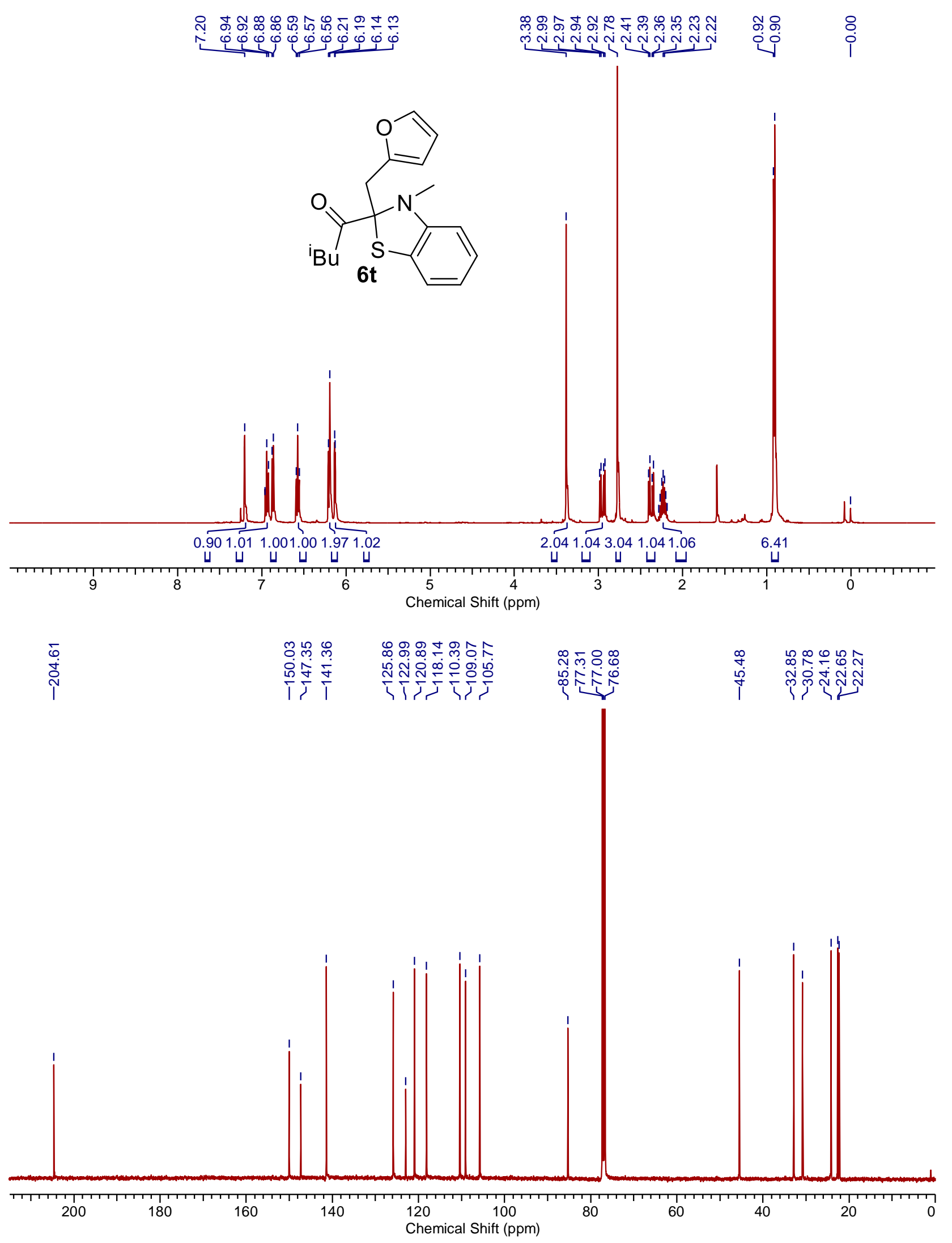




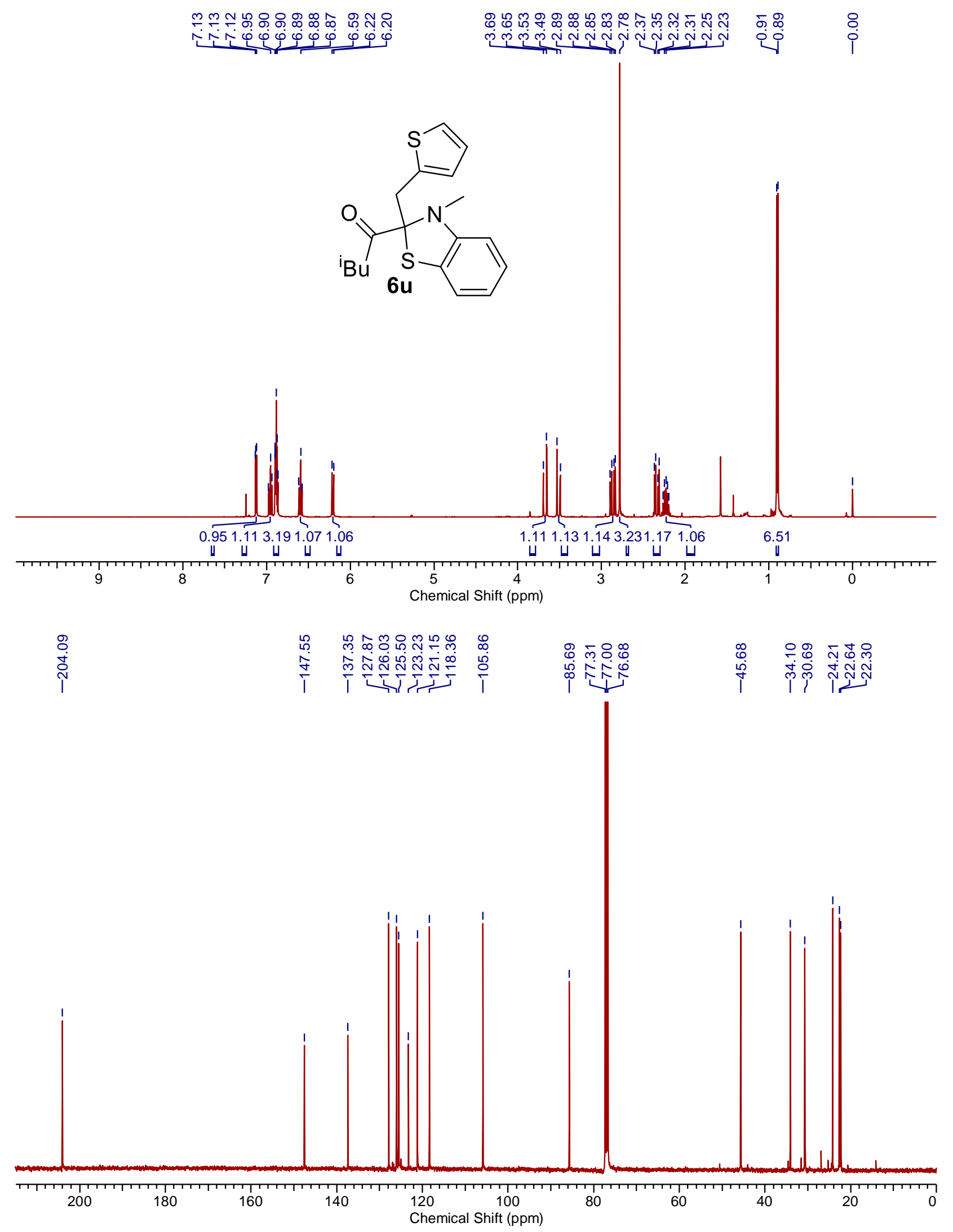



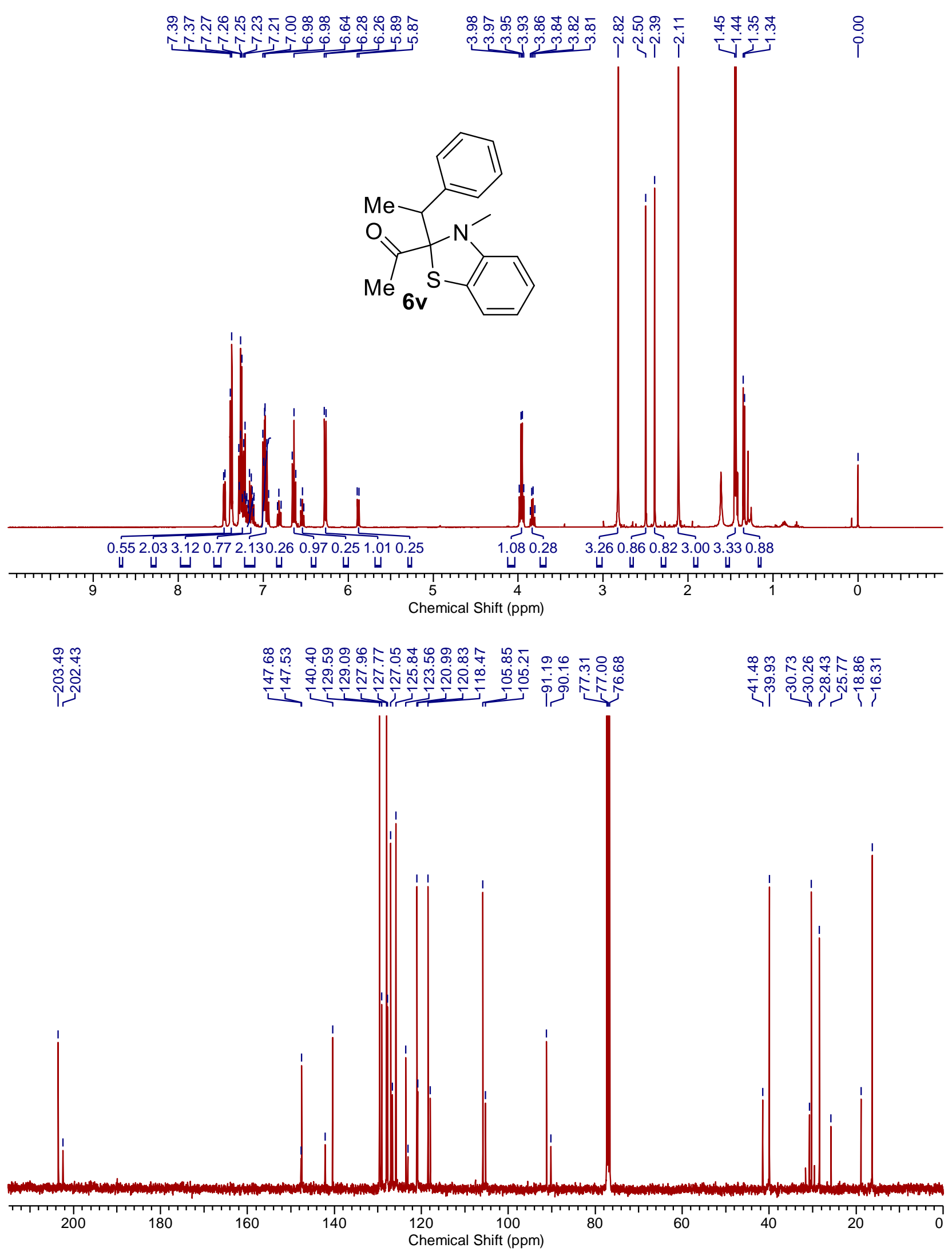


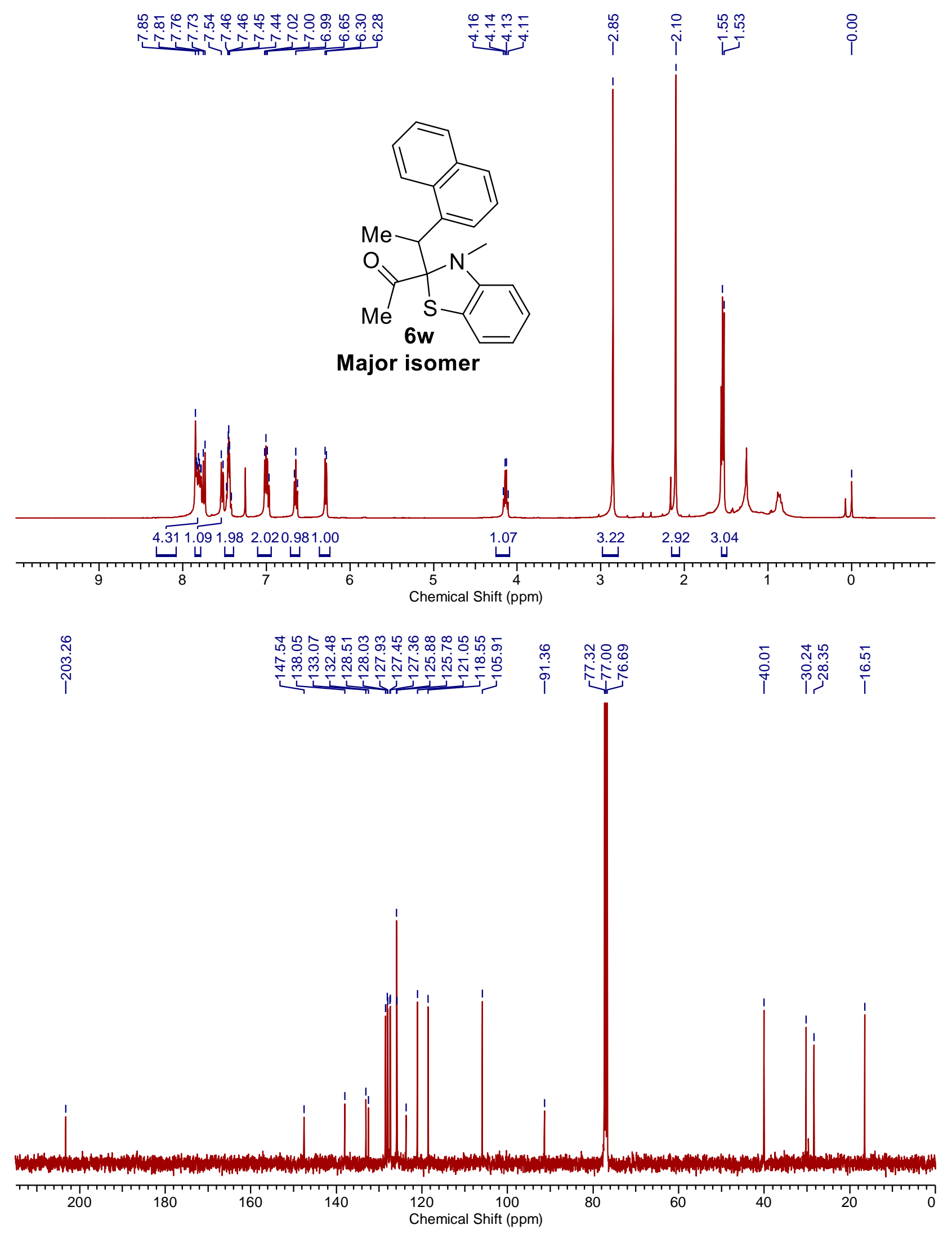




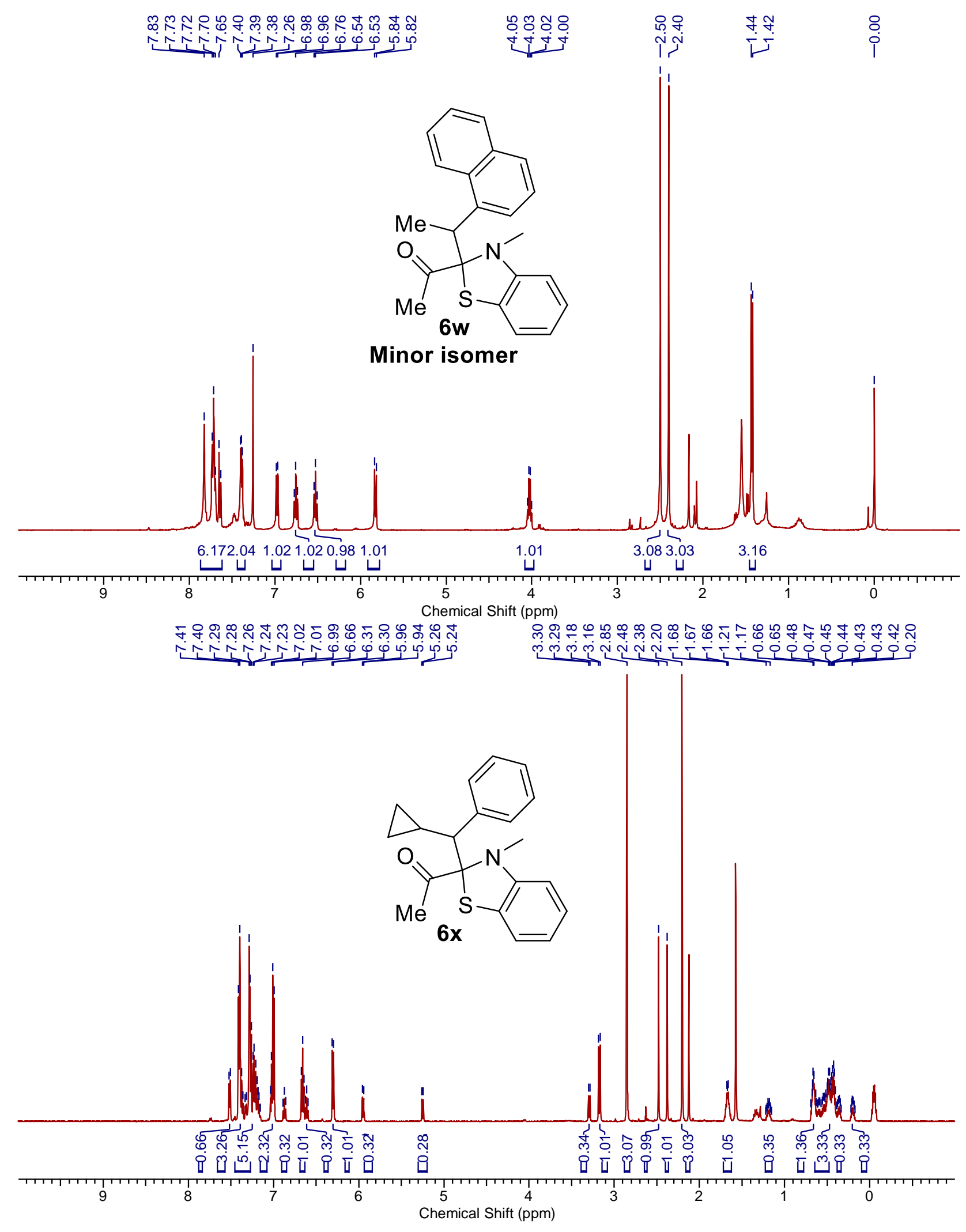



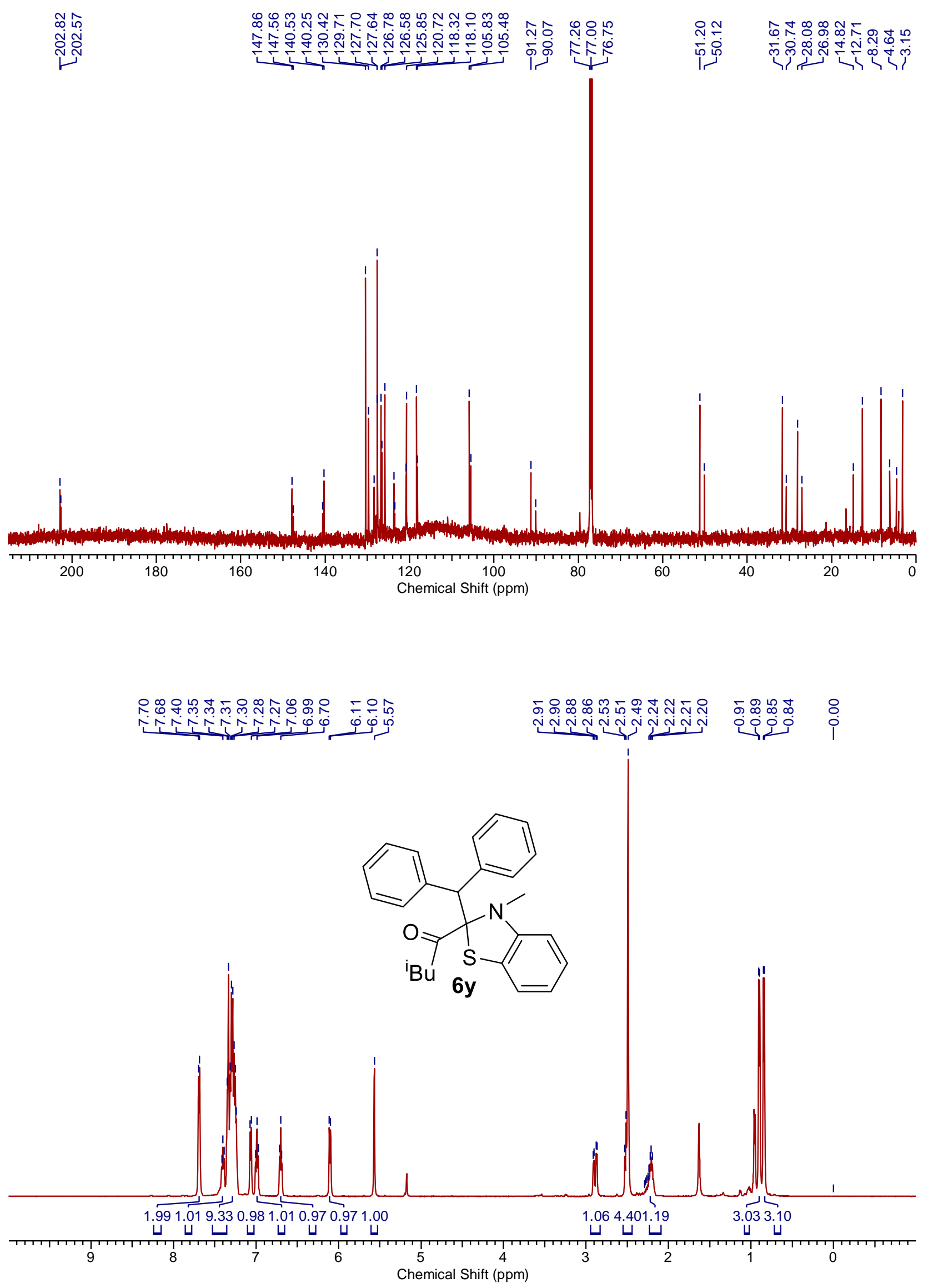

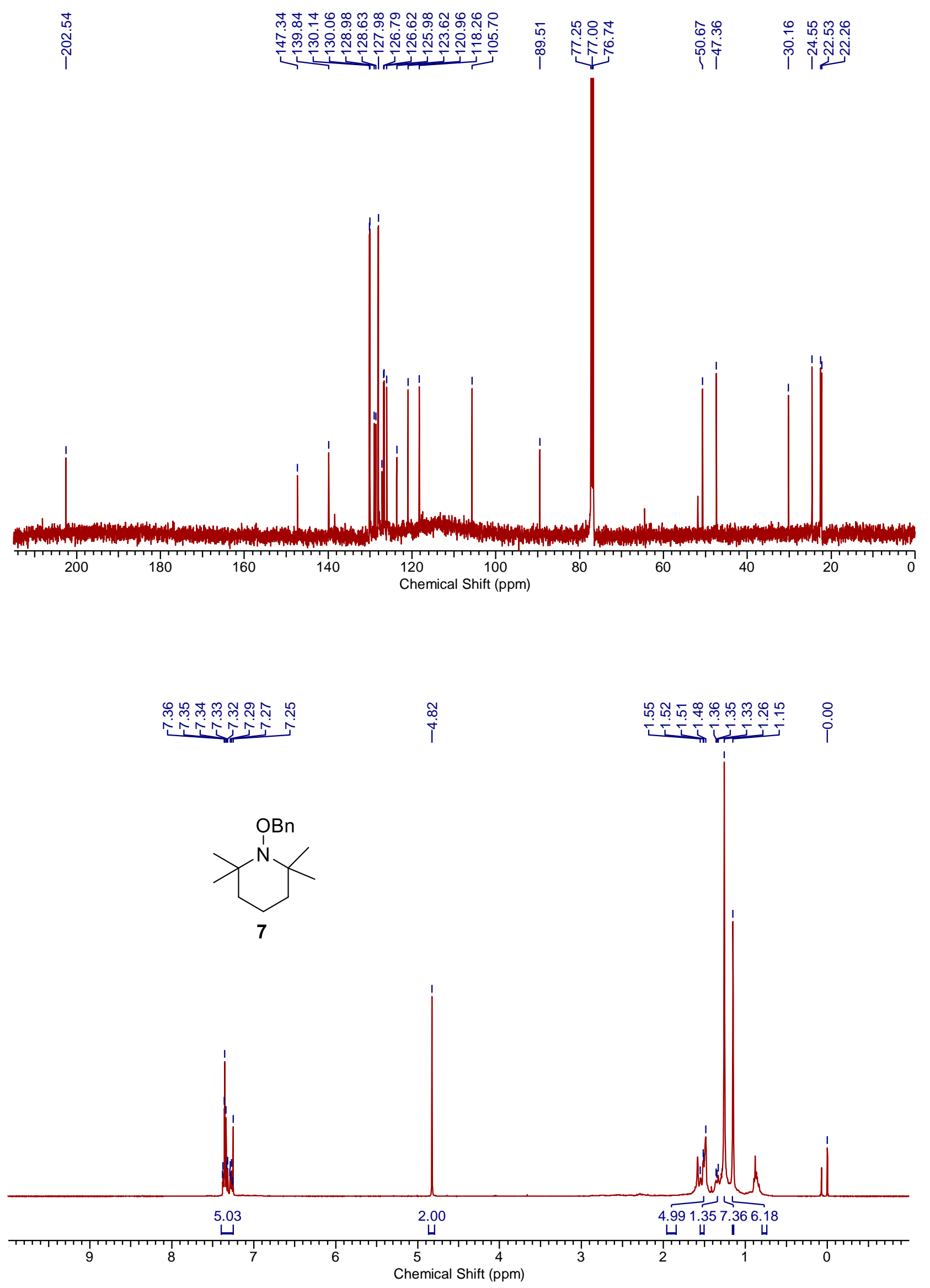

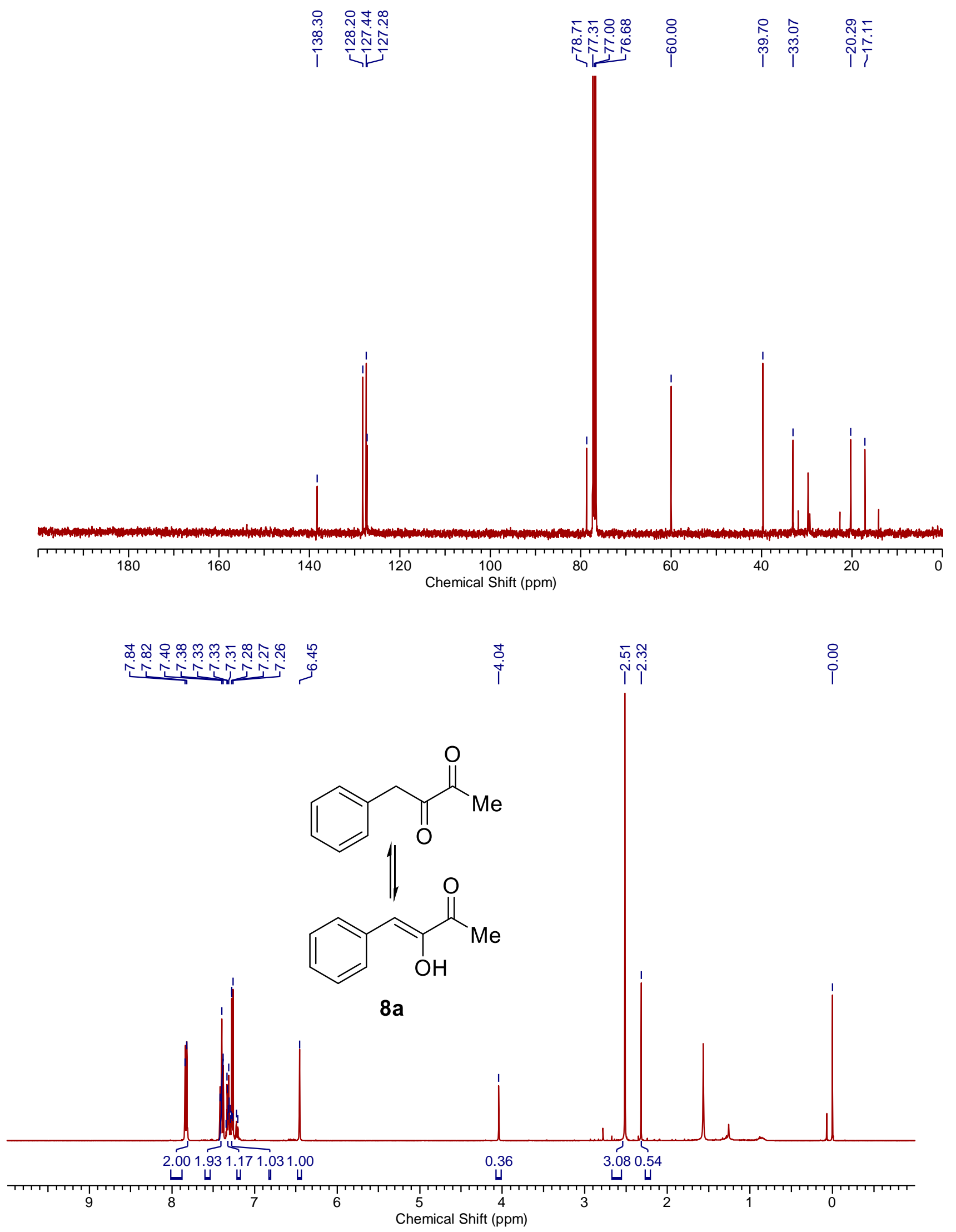

keto:enol $=0.18: 1.0$ 
<smiles>COc1ccc(C(=O)C(=O)C(=O)c2ccc(C(=O)C(O)=C(c3ccccc3)c3ccccc3)cc2)cc1</smiles>

$8 b$

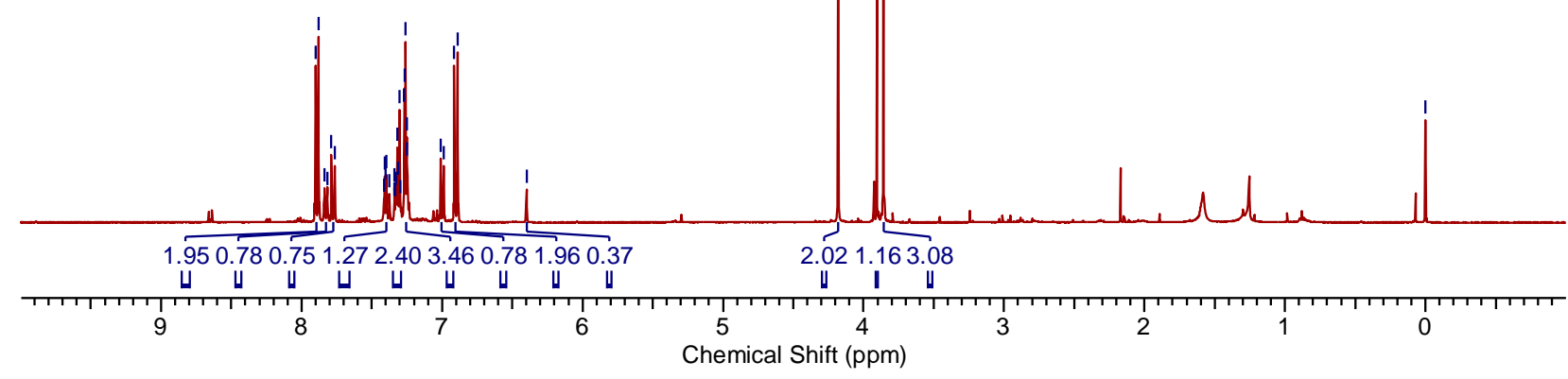

keto:enol $=1.0: 0.37$

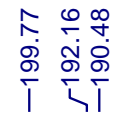

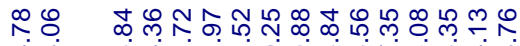

过

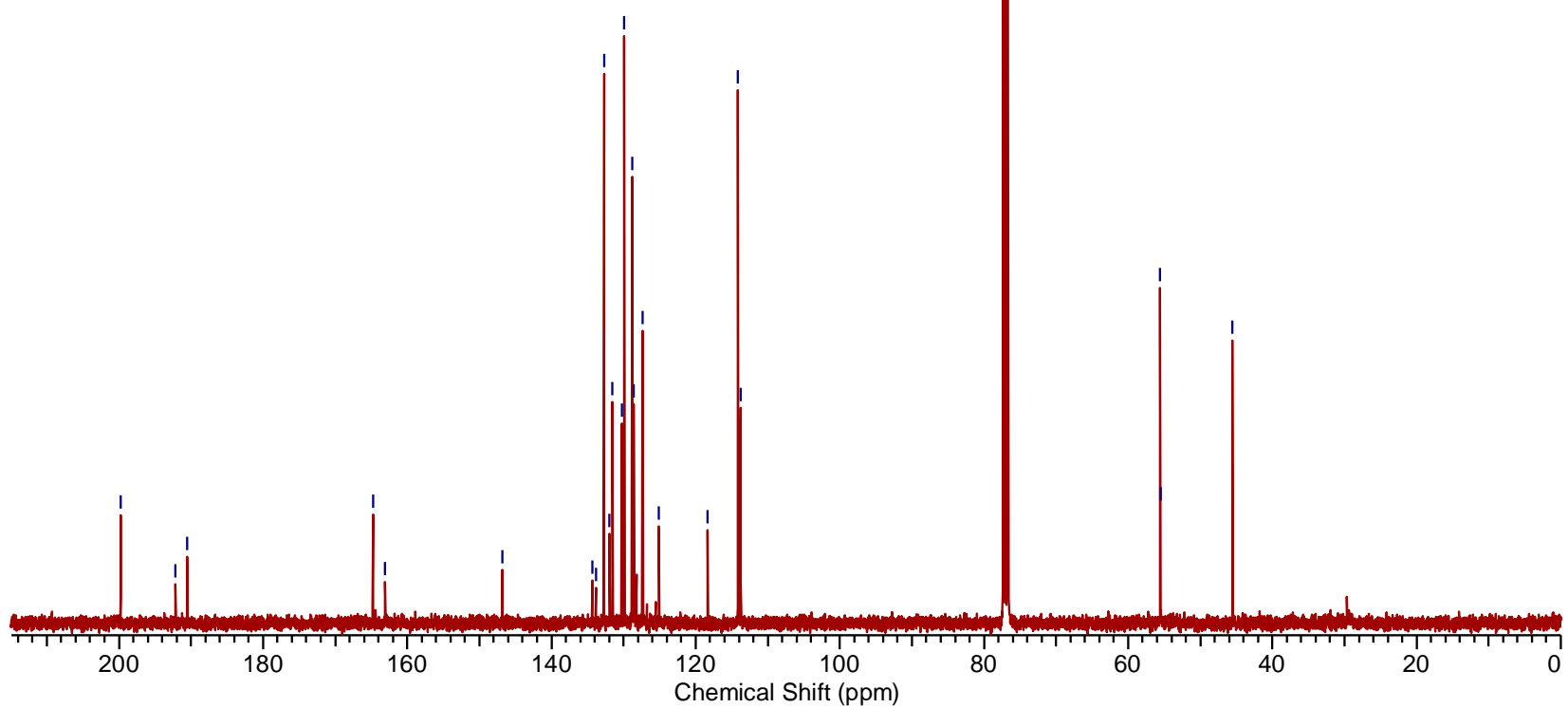


<smiles>CC(C)CC(=O)/C(O)=C/c1ccco1</smiles>

8c

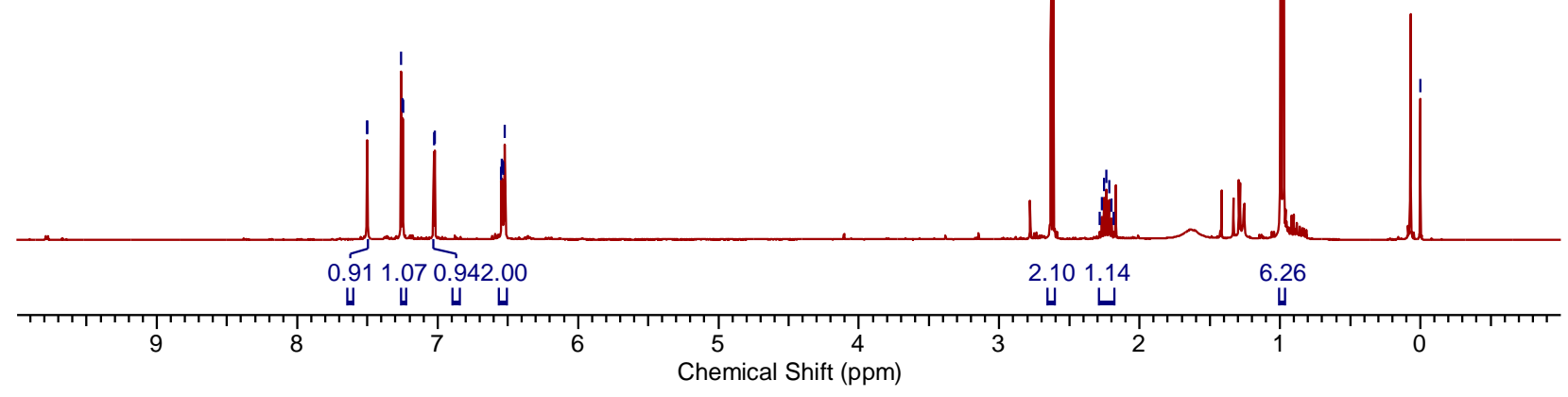

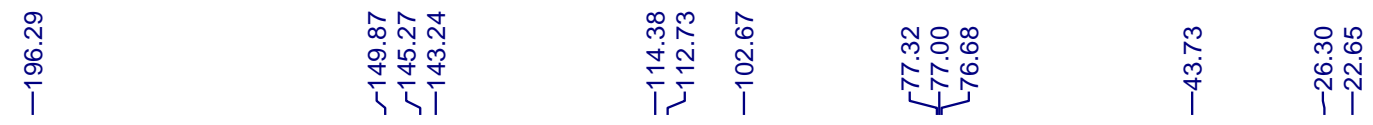

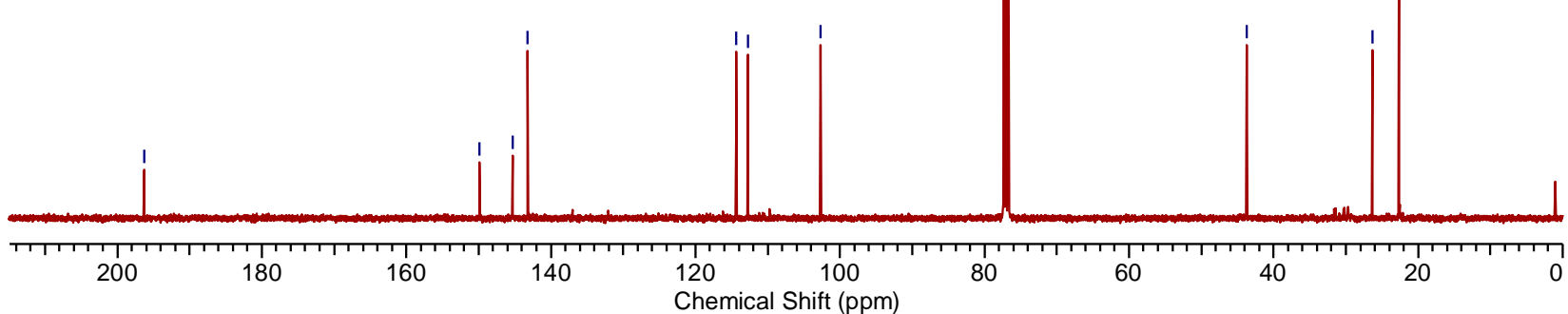



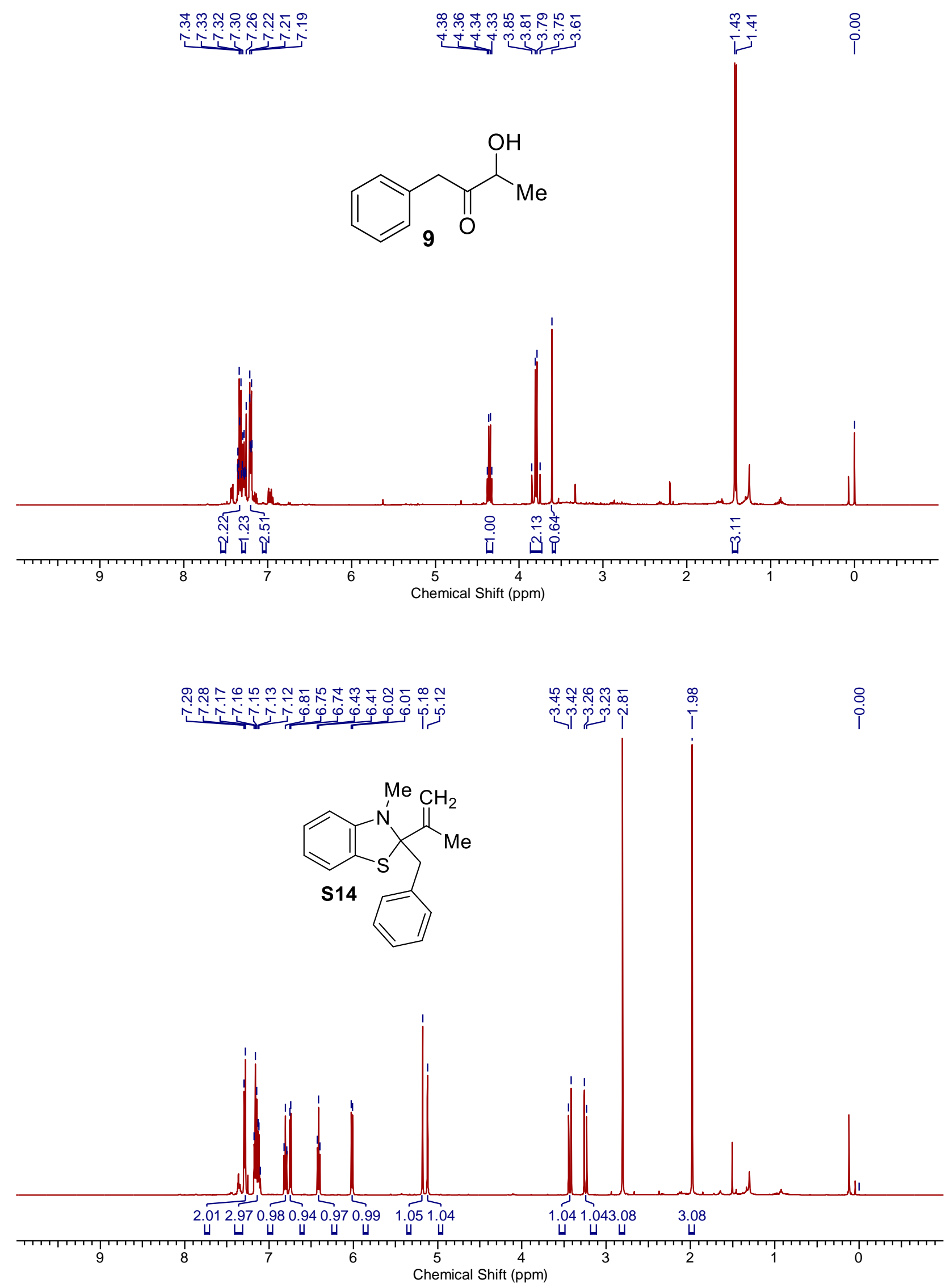

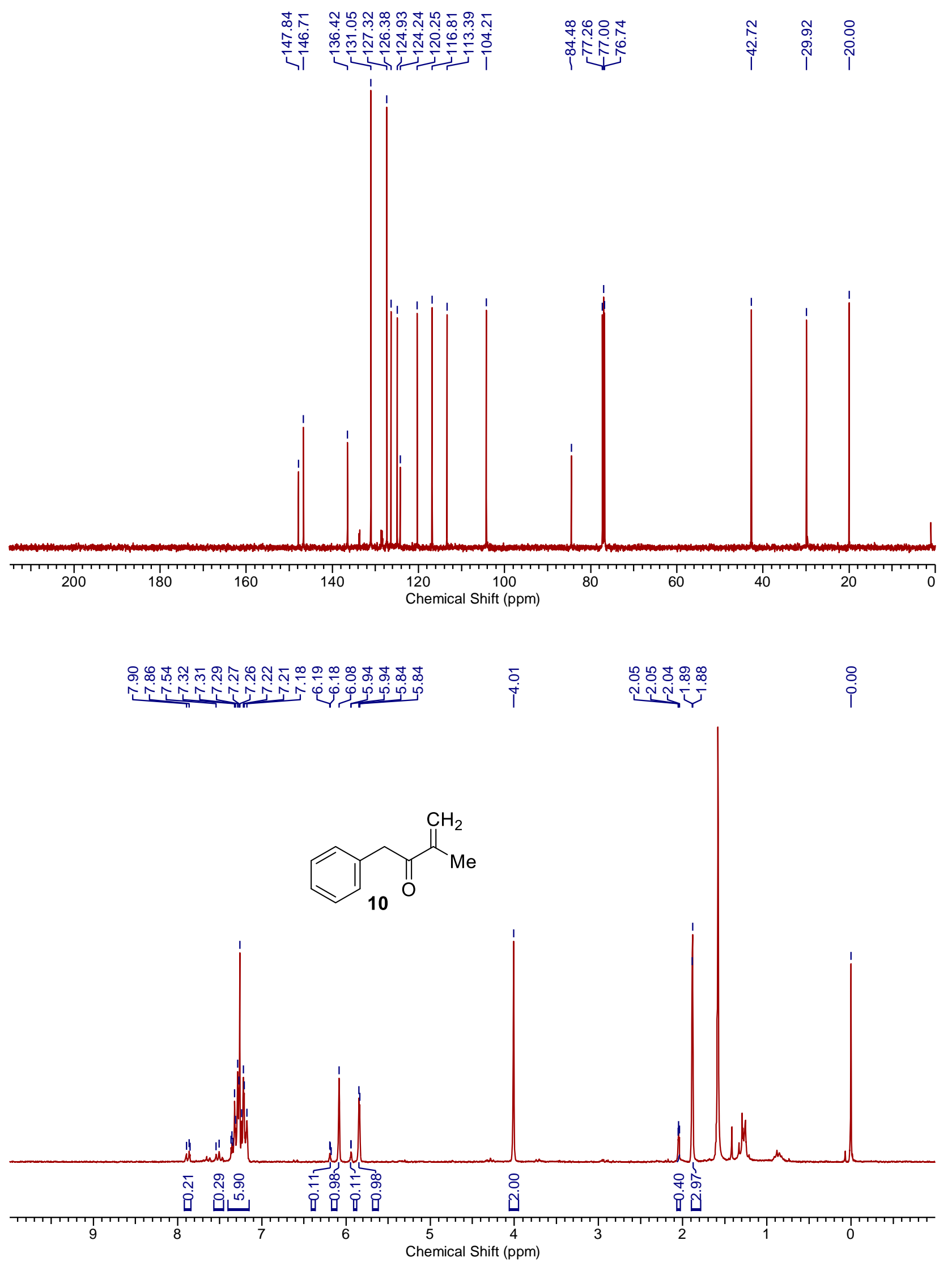


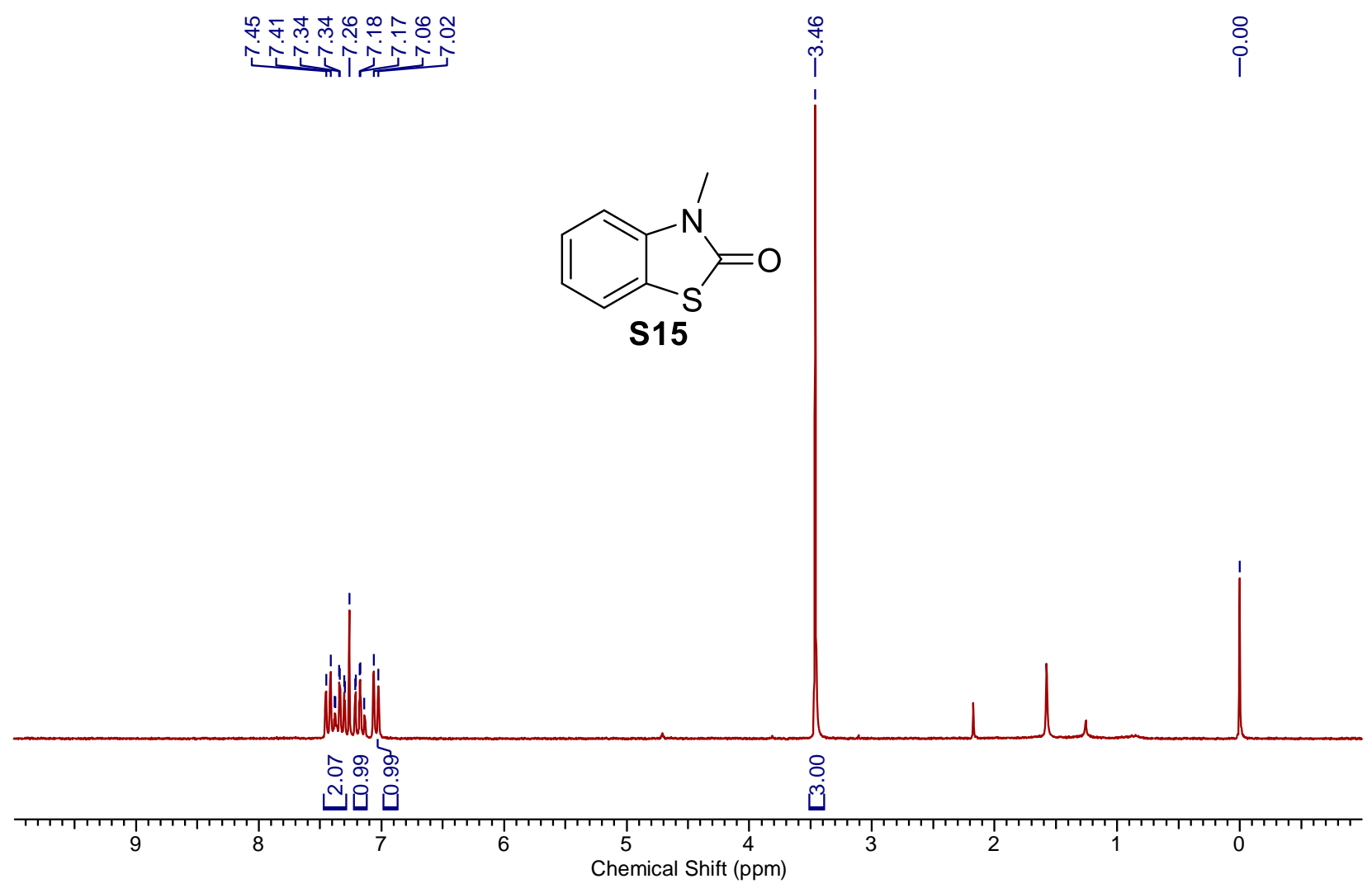

